\title{
Physical controls on copepod aggregations in the Gulf of Maine
}

\author{
by \\ Nicholas W. Woods \\ B.S. Physics, University of North Carolina, Wilmington \\ Submitted in partial fulfillment of the \\ requirements for the degree of \\ Doctor of Philosophy in Physical Oceanography \\ at the \\ MASSACHUSETTS INSTITUTE OF TECHNOLOGY \\ and the \\ WOODS HOLE OCEANOGRAPHIC INSTITUTION \\ June 2013 \\ (C) Nicholas W. Woods, 2013
}

The author hereby grants to MIT and to WHOI permission to reproduce and distribute publicly paper and electronic copies of this thesis document in whole or in part.

Author

Joint Program in Physical Oceanography

Massachusetts Institute of Technology

Woods Hole Oceanographic Institution

May 5, 2013

Certified by

David Fratantoni

Associate Scientist with Tenure, WHOI

Thesis Supervisor

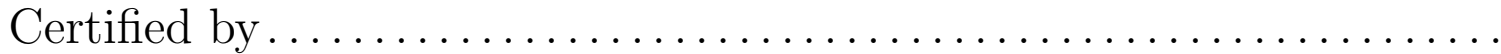

Glenn Flierl

Professor, MIT

Thesis Supervisor

Accepted by

Glenn Flierl

Chair, Joint Committee for Physical Oceanography 


\title{
Physical controls on copepod aggregations in the Gulf of Maine
}

by

\author{
Nicholas W. Woods
}

\author{
Submitted to the Joint Program in Physical Oceanography - Massachusetts \\ Institute of Technology / Woods Hole Oceanographic Institution \\ on May 5, 2013, in partial fulfillment of the \\ requirements for the degree of \\ Doctor of Philosophy in Physical Oceanography
}

\begin{abstract}
This thesis explores the role that the circulation in the Gulf of Maine (GOM) plays in determining the distribution of dense aggregations of copepods. These aggregations are an important part of the marine ecosystem, especially for endangered North Atlantic right whales. Certain ocean processes may generate dense copepod aggregations, while others may destroy them; this thesis looks at how different characteristics of the GOM circulation fit into these two categories.

The first part of the thesis investigates a hypothetical aggregation mechanism in which frontal circulation interacts with copepod behavior to generate a dense patch of copepods. The first two chapters of this thesis address this mechanism in the context of coastal river plumes and salinity fronts. One chapter describes the characteristics and variability of coastal freshwater and salinity fronts using a historical dataset and a realistic numerical model. The seasonal variability of freshwater is tied in part to seasonality in river discharge, while variability on shorter time scales in the frontal position is related to wind stress. Another chapter applies the hypothetical mechanism to idealized river plumes using a suite of numerical models. The structure of the plume and plume-relative circulation change the resulting copepod aggregation from what is expected from the hypothetical mechanism.

The second part of the thesis discusses the GOM circulation and how it may eliminate copepod patches. The summertime mean surface circulation and eddy kinetic energy are computed from a Lagrangian drifter dataset and an adaptive technique that allows for higher spatial resolution while also keeping uncertainty low. Eddy diffusivity is also computed over different regions of the GOM in an attempt to quantify the spreading of a patch of copepods, and is found to be lower near the coast where right whales are often found feeding on copepod patches. In the next chapter, a numerical drifter dataset is used to understand how the results of the previous chapter depend upon the quantity of observations. It is found that the uncertainty in esti-
\end{abstract}


mating eddy diffusivity is tightly coupled to the number of drifters in the calculation.

Thesis Supervisor: David Fratantoni

Title: Associate Scientist with Tenure, WHOI

Thesis Supervisor: Glenn Flierl

Title: Professor, MIT 


\section{Acknowledgments}

There are many people without whom I could not have gotten through this work. Thanks to my advisor, Dave Fratantoni, for being so patient and supportive through the years. Dave's advice, guidance, and most of all sense of humor made my thesis work not only enjoyable, but possible at all. Thanks also to my second advisor, Glenn Flierl. Glenn's guidance over the end of my thesis, in particular the idealized modeling and interpretation, has been inspiring and makes this entire work possible. The other members of my committee were also very influential. Steve Lentz always kept me focused on the end goal, provided detailed feedback on anything I sent to him, and was always ready to help with any problem I brought him. Mark Baumgartner's insight into the biological side of my work gave perspective and context when I needed it most. Rubao Ji gave me incredibly useful advice in several aspects of the work, and discussions with him always left me with lots of interesting ideas. Glen Gawarkiewicz, when he wasn't draining jump shots over me on the basketball court, provided me with lots of helpful advice not only about the specific science in my thesis but in general about its context, as well as my career and anything on which I needed advice, and is a saint for agreeing to chair my defense.

Lots of other folks at MIT, WHOI, and elsewhere have been extremely helpful during this process. The staff in academic programs-Ronnie, Julia, Marsha, Trisha, and Lea-make the world go around as far as I can tell. Other members of the PO department, in particular Karl Helfrich and Anthony Kirincich-have always been available to answer any questions I had. Thanks especially to Claudia Cenedese, Larry Pratt, Jim Price, and Fiamma Straneo for letting me be a teaching assistant for their classes. Thanks to Paul Fucile and Craig Marquette for their expertise and technical assistance. The PO front office staff-especially Annie Doucette, Hazel Salazar, and Deb Taylor-helped me with so many different things that I could never have done on my own. Thanks to Mark Merrifield and Janet Becker for all their help, advice, support, and intense fantasy football and March Madness bracket battles. I 
greatly appreciate getting to participate in a couple of research cruises with Gareth Lawson; thanks for letting me stare at grainy krill pictures for a couple of weeks. Also, I need to thank Changsheng Chen at UMass-Dartmouth and Ruoying He at NC State for the use of their numerical model output, Jim Manning for the drifter dataset, and Jim Churchill at WHOI for his help with the particle tracking software.

The other PO students in the Joint Program have been good friends during my time here, and I've learned as much (maybe more?) from them than anyone over the years. Thanks in particular to my classmates, Sophie Clayton, Wilken-Jon Von Appen, Julian Schanze, and Ru Chen for helping me get through all the classes we took together. My officemates, Rachel Horwitz and Beatriz Pen̋a Molino, were the absolute best people to hang with at work every day. I've also made some really close friends during my time in the JP. TJ and Jamie, thanks for the laughs and good times. Ben and John, thanks for all the help in the lab and steak-and-cheese sandwiches from Steve's.

My family has been extremely supportive throughout my life, and are without a doubt one of the biggest reasons I've gotten to this point. Mom, thank you for being so supportive and understanding through my time so far from home, thank you for all the care packages and sweet notes and cards, you always know how to brighten my day. Dad, your comments helped immensely in the writing of this thesis, and your hypothesis about the "copepod nightclub" where all the copepods congregated is the most unique and innovative theory yet proposed. Russell and Colie, thanks for being great brothers. Also thank you to all my cousins for being so fun!

I'd like to thank our pets Henry, Haley, and Iker, for always knowing when a snuggle, cuddle, or wet dog nose is most needed. Also, thanks for the random semicolons at various places in MATLAB code that take hours to find. Lastly, I want to thank Sophie, who has supported me every single day through the last few years with very little received in return. I can't express the gratitude I feel or how amazing you've been through this process. You've made this whole thing worthwhile; I really am the luckiest guy in the world. 
This work was supported by the WHOI Coastal Ocean Institute Graduate Student Fellowship and Student Research Award, the WHOI Academic Programs Office, the NOAA National Marine Fisheries Service Northeast Fisheries Science Center (NOAA Cooperative Agreement NA09OAR4320129), and the Office of Naval Research Marine Mammals and Biology Program (Grant N00014-12-1-0208). 


\section{Contents}

1 Introduction 13

1.1 Calanus finmarchicus and the South Channel Ocean Productivity Experiment $($ SCOPEX $) \ldots \ldots \ldots \ldots \ldots$

1.2 The Gulf of Maine circulation . . . . . . . . . . . . . . . 16

1.3 The contents of this thesis $\ldots \ldots \ldots \ldots$

2 Surface freshwater in the Southwester Gulf of Maine 23

2.1 Introduction . . . . . . . . . . . . . . . . . 23

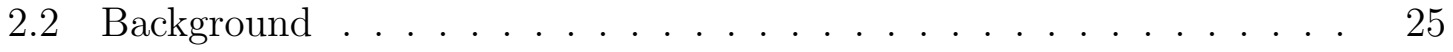

2.2 .1 Seasonal variability . . . . . . . . . . . . . . 26

2.2 .2 Shorter time scales . . . . . . . . . . . . . . . . 27

2.3 Seasonal cycle of coastal freshwater . . . . . . . . . . . . . . 29

2.3.1 Shipboard TSG Dataset . . . . . . . . . . . . . . . . . 29

2.3.2 Seasonal evolution of the coastal freshwater . . . . . . . . 29

2.3.3 River discharge vs. observed sea surface salinity . . . . . . . 36

2.3.4 Wind forcing vs. observed sea surface salinity . . . . . . . 40

2.3.5 The salinity front and its response to wind forcing and river discharge . . . . . . . . . . . . . . . . . 41

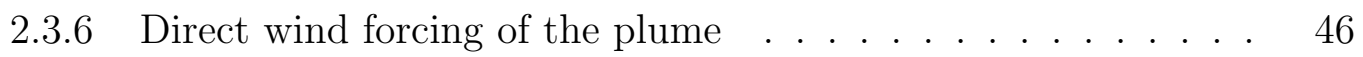

2.4 Realistic Numerical Model of the Plume and Wind Forcing Theory . 47

2.4.1 Model Description . . . . . . . . . . . . . . . . . . . 48

2.4.2 Comparison between model and surface dataset . . . . . . . 49 
2.4.3 Theory for wind forcing of buoyant plume . . . . . . . . 50

2.4.4 Wind effect on plume: Application of theory to model . . . . 59

2.5 Discussion and Conclusions _. . . . . . . . . . . . . . 63

\section{Idealized study of copepod aggregation at the edge of a buoyant} $\begin{array}{ll}\text { plume } & 69\end{array}$

3.1 Introduction . . . . . . . . . . . . . . . . . . . . 69

3.2 Plumes and convergence in the southwestern GOM _ . . . . . 72

3.2.1 Observations of a freshwater plume and copepod behavior in the GSC-autonomous underwater gliders . . . . . . . . . 73

3.2.2 Propagation of the plume (and associated convergence) far downstreamFVCOM simulations . . . . . . . . . . . . . 76

3.3 Methods . . . . . . . . . . . . . . . . . . . . . . . 77

3.3.1 2.5 layer plume and copepod concentration model . . . . . . 77

3.3.2 ROMS plume model . . . . . . . . . . . . . . . . . . . . . . 79

3.3.3 LTRANS particle tracking . . . . . . . . . . . . . . . 81

3.4 Layer model and ROMS results . . . . . . . . . . . . . . . 83

3.4 .1 Layer model validation . . . . . . . . . . . . . . . . . 83

3.4.2 Plume-relative circulation . . . . . . . . . . . . 86

3.4.3 Layer model - how does the plume affect the resulting copepod distribution? . . . . . . . . . . . . . . . . . . 93

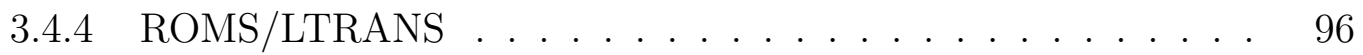

3.5 Idealizing the plume front/copepod interaction . . . . . . . . . 107

3.5.1 Scaling for expected concentration increase: one-dimensional "plow" model . . . . . . . . . . . . . . . . . . . . . . . 108

3.5.2 Two-dimensional "plow": a curved front with front-relative circulation . . . . . . . . . . . . . . . 110

3.5 .3 Slowing "plow" . . . . . . . . . . . . . . . . . . . . . . 113

3.6 Discussion/Conclusion . . . . . . . . . . . . . . . . . . . . 115 
4 Surface currents and eddy diffusivity estimates in the Gulf of Maine119

4.1 Introduction . . . . . . . . . . . . . . . . . . . . 119

4.2 The drifter dataset . . . . . . . . . . . . . . . . . . . . 122

4.2.1 GOM Drifter Observations . . . . . . . . . . . . . . . . . 122

4.3 Methods . . . . . . . . . . . . . . . . . . 123

4.3.1 Computing summertime mean fields . . . . . . . . . . . . . . . 123

4.3.2 Computing diffusivity from single-particle statistics . . . . . . 124

4.3.3 Two-particle dispersion and diffusivity . . . . . . . . . . . . 126

4.4 Results-Observations . . . . . . . . . . . . . . . . 127

4.4.1 Mean flow and EKE, $0.1^{\circ} \times 0.1^{\circ}$ grid . . . . . . . . . . . . 127

$4.4 .2 \kappa$ over the entire GOM . . . . . . . . . . . . . . . 133

4.4 .3 Pair dispersion . . . . . . . . . . . . . . . . 135

4.4.4 Three sub-regions: Interior GOM, Coastal Current, and Georges Bank ...................... 141

4.4.5 Comments on using east-north coordinates to estimate $\kappa$ in coastal regions . . . . . . . . . . . . . . . . . 143

4.5 Discussion and conclusion . . . . . . . . . . . . . . . . 145

5 Evaluating Lagrangian methods in the Gulf of Maine 151

5.1 Introduction . . . . . . . . . . . . . . . . . . 151

5.2 The drifter data, model, and model drifters . . . . . . . . . . . . . 153

5.2.1 GOM Drifter Observations . . . . . . . . . . . . . . 153

5.2.2 FVCOM simulations of the GOM . . . . . . . . . . . 153

5.2.3 Particle tracking in FVCOM . . . . . . . . . . . . . . 155

5.3 Methods . . . . . . . . . . . . . . . . 157

5.3.1 Comparing mean fields computed via standard gridding and adaptive clusters . . . . . . . . . . . . . . 157

5.3.2 Computing diffusivity from single-particle and two- particle statistics . . . . . . . . . . . . . . . . 158 
5.3.3 Error estimates by bootstrapping . . . . . . . . . . . . . 159

5.3 .4 Derivation of $\kappa_{G F} \ldots \ldots \ldots \ldots \ldots \ldots \ldots$

5.4 Standard gridding vs. adaptive clusters . . . . . . . . . . . . . 161

5.5 Evaluating the model, model drifters, and observational processing techniques . . . . . . . . . . . . . . . . . . . 164

5.5.1 Mean flow and EKE differences . . . . . . . . . . 166

5.5.2 Testing the particle tracking spatial interpolation . . . . . 169

5.5.3 Testing the effect of low-pass filtering the drifter positions . . 171

5.5.4 Testing the effect of computing the drifter velocity from 1st differences . . . . . . . . . . . . . . . . . . . 172

$5.6 \kappa$ derived from model drifters vs observations . . . . . . . . . 175

$5.6 .1 \kappa$ over the entire GOM, model drifters . . . . . . . . . 175

5.6.2 Pair dispersion and eddy diffusivity . . . . . . . . . . 177

5.6.3 Three sub-regions: Interior GOM, Coastal Current, and Georges Bank . . . . . . . . . . . . . . . . . . 179

5.6.4 Diffusivity from Green's Functions _. . . . . . . . . . . . 184

5.7 Diffusivity uncertainty estimates . . . . . . . . . . . . 185

5.7.1 Single-particle, all GOM . . . . . . . . . . . . . . 186

5.7 .2 Single-particle, three sub-regions $\ldots \ldots \ldots \ldots$

5.7 .3 Particle pairs . . . . . . . . . . . . . . . . . 193

5.8 Discussion and conclusion $\ldots \ldots \ldots \ldots \ldots$

6 Discussion and conclusions 201

6.1 Major contributions of this thesis . . . . . . . . . . . . . 201

6.2 Future work . . . . . . . . . . . . . . . . . . 203

6.3 Concluding remarks . . . . . . . . . . . . . . . 206 


\section{Chapter 1}

\section{Introduction}

Aggregation of prey in the ocean plays a vital role in food web ecology. Many predators may not be able to survive without prey aggregation. For example, sea lions, killer whales, and sea birds feed on schools of herring (Pitcher and Calkins (1981), Nøttestad and Similä (2001), Ostrand et al. (2004)), blue whales feed on swarms of krill (Schoenherr (1991)), and basking sharks, cod, haddock, and right and sei whales feed on highly concentrated patches of copepods (Sims and Quayle (1998), Wishner et al. (1995), Werner et al. (2003)). The processes that aggregate prey therefore play an important role in fisheries management and species conservation.

This thesis aims to understand the physical mechanisms that may be responsible for dense aggregations of the copepod Calanus finmarchicus in the Gulf of Maine (GOM), a semi-enclosed basin in the northwest Atlantic Ocean (figure 1-1). These aggregations are a food source for many species, including the endangered right whale (Eubalena glacialis), which return to the southwestern GOM every spring to forage. We approach the problem from two separate but related points of view. The first part of the thesis explores a mechanism through which ocean circulation may cause copepods to aggregate, while the second part of the thesis investigates the ways in which the flow and circulation may cause a patch of copepods to spread out. Both of these points of view are related to copepod patchiness, and are thus important in understanding the interaction between the flow and these important organisms. 
Before introducing the contents of this thesis, we discuss some of the past work on this problem, beginning with the South Channel Ocean Productivity Experiment (SCOPEX).

\subsection{Calanus finmarchicus and the South Channel Ocean Productivity Experiment (SCOPEX)}

Calanus finmarchicus is one of the two species of zooplankton that dominates the waters of the Gulf of Maine in the spring. Due to its relatively large size $(>3 \mathrm{~mm}$ as adults, Lalli and Parsons (1997)), C. finmarchicus is responsible for a large portion of the zooplankton biomass in the region. These organisms typically swim at speeds ranging the orders of 0.1-10 cm/s (Buchanan et al. (1982); Epstein and Beardsley (2001); Genin et al. (2005)), allowing them to swim effectively against vertical currents; however $C$. finmarchicus individuals can swim at speeds up to $80 \mathrm{~cm} / \mathrm{s}$ to avoid predators (Lenz et al. (2004)).

The life history of $C$. finmarchicus has been the topic of intensive investigation, which is reviewed in Tarrant et al. (2008) and summarized here. The distinguishing feature of $C$. finmarchicus life history is a period preceding adulthood known as diapause, in which the organisms enter a resting state. Individuals emerge from diapause in early winter, molt into adults, and mate (Durbin et al. (1997)). After hatching, the earliest nauplii stages do not feed, but the later nauplii and all copepodite stages feed on the spring phytoplankton bloom. In the later copepodite stages, individuals develop oil sacs, which reach their maximum size by the 5th copepodite stage (Baumgartner et al. (2007)). After this stage, a portion of the population migrates to depth and enters diapause, while the rest of the population molts into adults and mate again. In the Gulf of Maine two or more generations a year may occur (Durbin et al. (1997)). Eggs and nauplii stages of C. finmarchicus are an important source of food for cod and haddock (Kane, 1984), while later copepodite stages are the primary prey of right whales (Wishner et al. (1995)), thus patchiness in populations of $C$. fin- 
marchicus likely influences these important predators. Previous work, in particular the South Channel Ocean Productivity Experiment (SCOPEX) has elucidated some of the characteristics of the dense patches of $C$. finmarchicus in the southwestern GOM. SCOPEX (Wishner et al. (1995)) was an interdisciplinary project during the late 1980's and early 1990's that focused on the whale-copepod-circulation system. The SCOPEX team noted that the location of the densest patches occurred within the peak in $C$. finmarchicus abundance, which appeared to coincide with the salinity front on the eastern edge of the Channel (Wishner et al. (1995)). Using net-tows, the densest patches were found to range in size from 100-1000 m across, while the vertical extent of these patches is in the range of 1-10 m (Wishner et al. (1995), Baumgartner and Mate (2003)). Observations of peak density within a patch are in the range of $10^{3}-10^{4}$ copepods $/ \mathrm{m}^{3}$ (Wishner et al. (1995), Beardsley et al. (1996)), which is one to three orders-of-magnitude larger than typical values measured in the GOM in springtime (Epstein (1995), Baumgartner et al. (2003)). The temporal persistence of the patches is somewhere between hours and days, however what determines this time scale is unknown (M. Baumgartner, pers. comm.).

The leading hypothesis to explain high concentrations of copepods found in patches in the southwestern GOM after SCOPEX was that advection by circulation and coupled physical-biological mechanisms were likely to be aggregating copepods. The SCOPEX team ruled out in situ growth and social behavior as likely causes for the observed high-concentration patches in the southwestern GOM. The physical side, however, was not ruled out, and in fact some evidence for a coupled physicalbiological aggregating mechanism was observed (Epstein and Beardsley (2001)). Since SCOPEX, there have been no major advances in understanding how the circulation and copepod patches interact. This thesis attempts to understand what role the circulation may play in forming, maintaining, and destroying these dense patches. 


\subsection{The Gulf of Maine circulation}

The GOM has received attention from researchers due to its economically-important fisheries; some of this work is summarized here. The GOM resides on continental shelf in the northwest Atlantic Ocean (figure 1-1). It stretches from Cape Cod to Nova Scotia, and is separated from the northwest Atlantic by Georges Bank, a shallow embankment that is an important fishing ground (Fry (1988)). The interior GOM is shallower than $200 \mathrm{~m}$ depth except in the three deepest basins, Jordan, Wilkinson, and Georges.

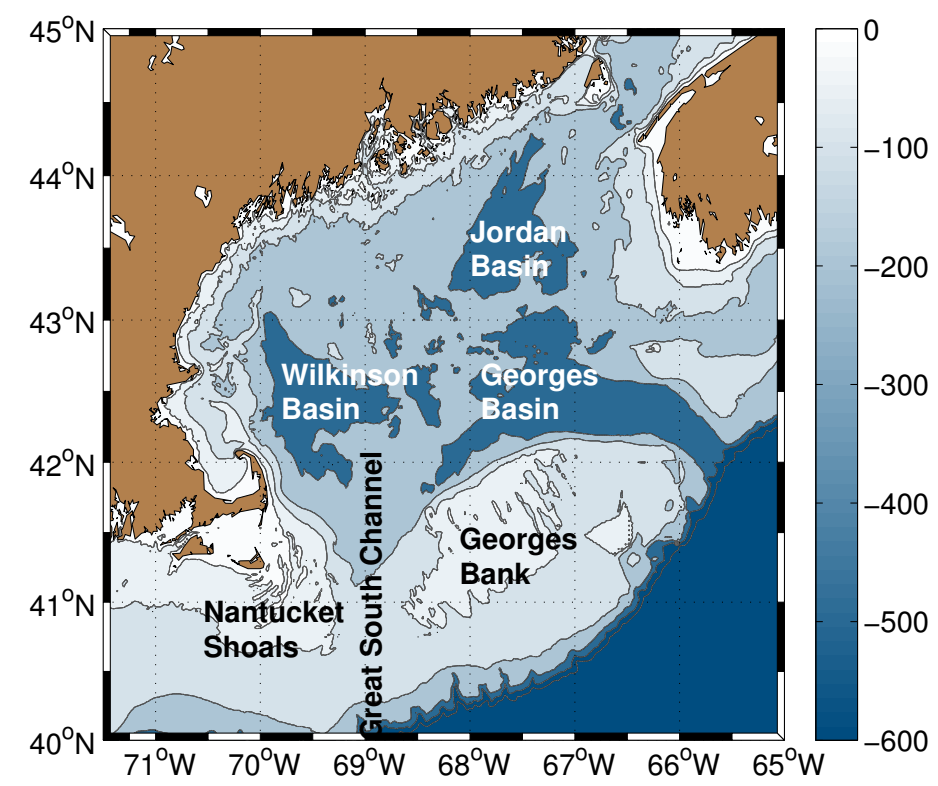

Figure 1-1: The Gulf of Maine. Colors are depths (in meters)

The circulation in the GOM is complicated, as there are many different physical processes at play. There is a coastal current that persists year-round (Pettigrew et al. (2005)), buoyant river plumes (Fong et al. (1997)), strong tides, steeply-sloped bathymetry, and associated tidally-rectified flows (Loder (1980)), wind-driven circulation (Hannah et al. (1997)), and internal waves (Haury et al. (1983)), among others. Some of these processes may be important to copepod aggregations. For example, work by Epstein and Beardsley (2001) has suggested that a buoyant river plume 


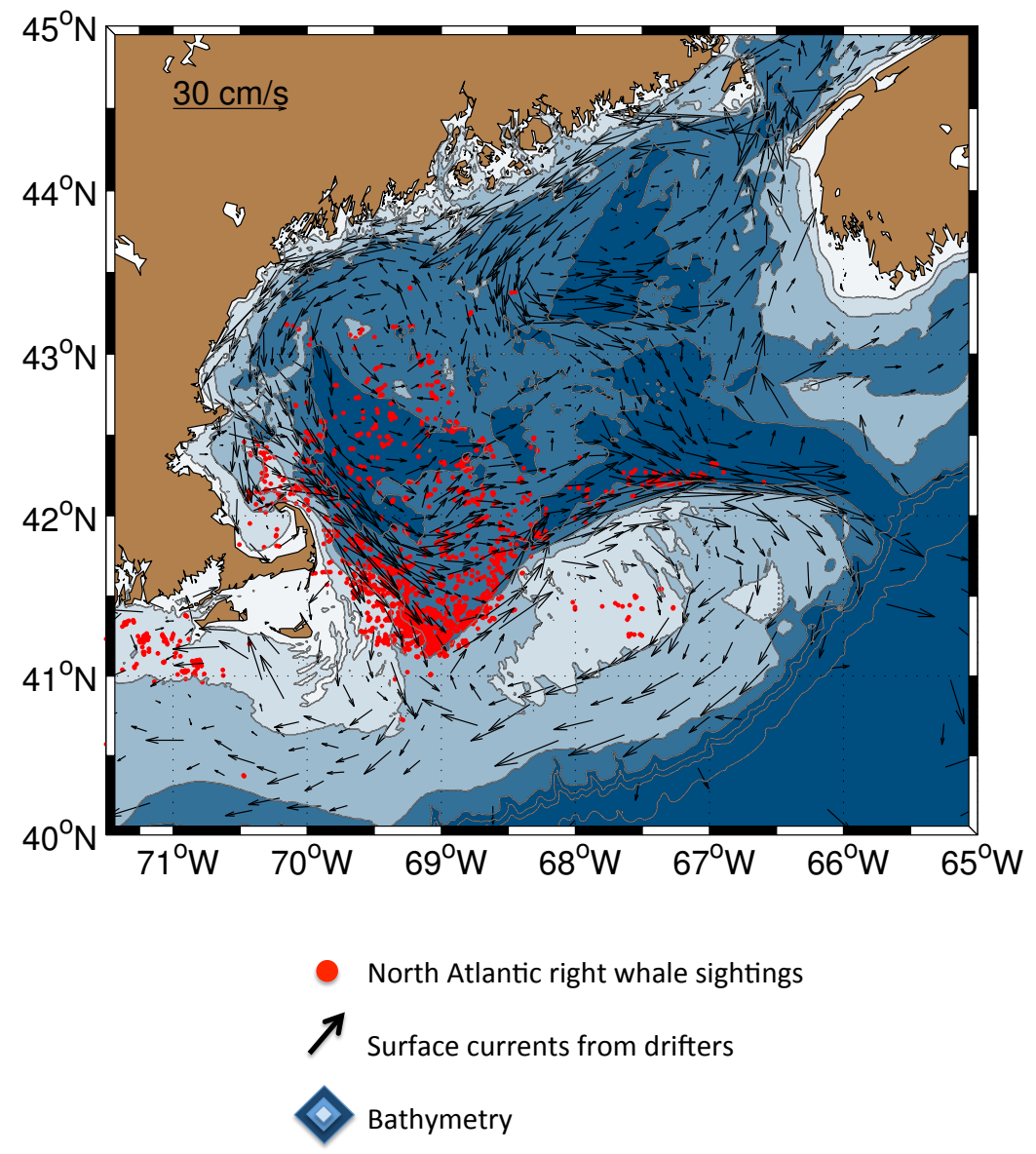

Figure 1-2: Gulf of Maine Circulation (surface drifter summertime climatology, data from Manning et al. (2009)) and North Atlantic right whale sightings (March-May)

may interact with copepod swimming behavior to generate a dense aggregation (see chapter 3 for a full explanation of the mechanism).

Despite this past work, the complex nature of the GOM circulation and how that relates to copepod aggregations is not well understood. Figure 1-2 shows the distribution of right whale observations in spring (April-June) from a historical database (OBIS SEAMAP, Halpin et al. (2006)), as well as the spring-summer surface circulation derived from surface drifters (using data from Manning et al. (2009), see chapter $4)$.

It is clear that the predominance of right whale sightings in spring occurs in the southwestern GOM. Since right whales come to this region to feed, figure 1-2 
implies that there are lots of dense copepod patches there. What is special about this region of the world's oceans? What physical processes are present there, and which are important? Does the proposed mechanism of Epstein and Beardsley (2001) actually occur in this region? Are there other characteristics of the flow that have not been considered that may relate to copepod aggregations? These are the questions addressed in this thesis.

\subsection{The contents of this thesis}

The first two chapters of the thesis address a mechanism through which a component of the flow in the southwestern GOM, specifically the buoyancy-driven flow associated with freshwater river plumes, aggregates copepods. In chapter 2, surface freshwater patterns and the corresponding salinity front east of Cape Cod, Massachusetts are investigated using NOAA shiptrack surface salinity data and a realistic numerical model. The goal of this work is to determine the spatial and temporal variability of this freshwater on scales relevant to dense copepod aggregations, including interannual, seasonal, and synoptic time scales. The strong seasonality of the freshwater there is partly due to river dicharge into the Gulf of Maine, which propagates downcoast as a part of the coastal current. Accordingly, the strength of the salinity front (defined as the cross-shore gradient in salinity at the boundary between fresh coastal water and salty interior water) and the salinity anomaly of the plume (the difference in salinity inshore and offshore of the salinity front) vary seasonally according to river freshwater discharge. The position of the salinity front, defined as the location of the maximum cross-shore salinity gradient, is advected on- or off-shore in response to downwelling and upwelling winds, respectively. A simple theory for the wind-driven response of a coastal freshwater plume is compared to an FVCOM simulation of the Gulf of Maine, which indicates that the theory exhibits some skill in predicting the width of the coastal freshwater. Including a parameterization for variable freshwater supply improves the theory's skill in predicting the cross-sectional area of the coastal 
freshwater, but the theory fails to predict variability in plume depth and density anomaly. This chapter provides one of the first comprehensive analyses targeted with understanding the salinity front in the southwestern GOM. In addition, it elucidates some weaknesses in the current theoretical understanding of how coastal freshwater plumes react to wind forcing (as outlined by Lentz (2004) and Moffat and Lentz (2011)).

Chapter 3 investigates the interaction of a buoyant plume with copepods using a series of idealized models. Conceptually, the density front associated with the nose of a buoyant plume can interact with copepod swimming behavior to generate a dense copepod patch in the following manner: as the plume propagates alongshore, it drives the ambient, heavier fluid downward. Copepods in the ambient fluid will feel the downward motion and swim against the downwelling to stay near the surface. As more and more copepods intersect the front and swim up, copepod density at the front will increase, forming a dense patch. This work expands upon this concept by applying it to idealized, three dimensional buoyant plume systems. A simple layer model, an idealized Regional Ocean Modeling System (ROMS) simulation, and a suite of conceptual models are used to investigate this problem. The front shape, plume-relative circulation, and copepod behaviors are all important in determining the resulting copepod distribution. The results of this chapter show the effect of including three-dimensional flow, which causes the copepod concentration to increase more slowly than that predicted by two-dimensional models. Also, copepods swimming to maintain a certain depth end up with a very different distribution from those that are sitting upon an isopycnal surface or those that move completely passively with the flow. It is clear from this work that copepod patchiness depends upon the dynamics of river plumes, and these results can be used to guide future field experiments.

Where the first part of the thesis addressed how the flow can generate dense copepod aggregations, the second part of the thesis addresses the inverse: how can the flow spread out a patch of copepods? To address this question, a surface drifter dataset is 
analyzed to attempt to understand patterns and variability in the surface circulation in the GOM from a Lagrangian point of view (chapter 4). Using Lagrangian drifters is particularly applicable because copepods, like most plankton, are largely at the whim of the horizontal flow, and thus may act similarly to drifters. Mean flow and eddy kinetic energy are computed on previously unresolved scales using a new, adaptive binning technique which also reduces uncertainty in the estimate. Single-particle eddy diffusivity is also calculated from the drifter tracks, which indicates that the diffusivity tensor is not isotropic in the GOM due to the limiting effect of the coastline. Spatial variability of the diffusivity tensor, as well as particle-pair diffusivity is also computed. While the coastal regions (those in which more whales and, by inference, more copepod patches) are found to be somewhat more diffusive, the effective resolution of the drifter data is shown to be at best roughly the scale of observed copepod patches. The calculations in this chapter therefore may be more indicative of larger scale processes that could be generating smaller scale motion in the flow important to copepod aggregations. Nonetheless, there is some indication from these results that regions where right whales are more often observed feeding on copepods are those in which the diffusivity is smaller.

Chapter 5 uses numerical drifters in a realistic simulation of the GOM (Finite Volume Coastal Ocean Model, FVCOM) to investigate the sensitivities of Lagrangian analysis. The model, model drifters, and observations are all compared to one another to validate the adaptive clustering techniques, data processing methods, and the uncertainty in calculating Lagrangian statistics from a small number of drifters. The methods used in processing the data and computing mean and EKE fields are shown to have some errors associated with them, but these errors are small $(<20 \%)$ over most of the GOM, allowing for definitive statements about the circulation to be made on previously unresolved spatial scales. The uncertainty on single-particle diffusivity is found to depend crucially upon the number of drifters used in the calculation, consistent with other studies in other regions. The uncertainty in the particle-pair diffusivity is also quantified for the first time in this work. While there are enough 
drifters in the observational dataset to make concrete statements about the GOMscale eddy diffusivity, it proves difficult to make detailed estimates about the spatial variability of this important quantity. Of the various results of this chapter, perhaps the most compelling is the need for more detailed and comprehensive Lagrangian data in the GOM in order to address dispersion and diffusion relevant to copepod patches.

The final thesis chapter will serve as a conclusion. The important contributions of this thesis will be summarized there, and some possible future work will be discussed. 


\section{Chapter 2}

\section{Surface freshwater in the Southwestern Gulf of Maine}

\section{$2.1 \quad$ Introduction}

Coastal freshwater plumes are an important feature commonly found in the coastal ocean (Shcherbina and Gawarkiewicz, 2008a). Accordingly, many theoretical and observational studies have focused on describing the governing dynamics of these systems (e.g. Lentz and Helfrich (2002)). Buoyant coastal currents are found where freshwater impinges on the shelf from a river or estuary; examples include the Chesapeake Bay (Lentz and Largier (2006),) Delaware Bay (Whitney and Garvine (2005)), the Western Adriatic (Magaldi et al. (2010)), East Greenland (Sutherland and Pickart (2008)), and Western Gulf of Maine (Fong et al. (1997)). The role of wind forcing has been shown to play a role in determining the structure of freshwater plumes (e.g. Chao (1987), Fong et al. (1997), Yankovsky and Chapman (1997), Fong and Geyer (2001), Lentz (2004), Lentz and Largier (2006), Magaldi et al. (2010)).

Herein, the coastal freshwater east of Cape Cod is investigated using surface observations and a realistic numerical model. The goal of this work is to describe the spatial and temporal variability of freshwater along the coast of Cape Cod on scales relevant to copepod aggregations. Epstein and Beardsley (2001) noted that obser- 
vations of North Atlantic right whales in spring 1989 occurred at the location of a salinity front separating fresh inshore water from salty offshore water, and discussed a model by which copepods may aggregate at this location. Calanus finmarchicus, the species of concern here, develop and mature over the course of the spring season in response to the spring phytoplankton bloom (Baumgartner et al. (2007), Tarrant et al. (2008)), so the first part of this work will focus on the seasonal cycle of coastal freshwater. On shorter time scales (a few days to a week), persistent dense patches of copepods are targeted by right whales and other organisms for fodder (what sets this time scale is unknown (M. Baumgartner, personal communication)). Studies of freshwater plumes indicate that wind forcing can cause variability on this range of time scales (Fong et al. (1997), Lentz (2004)), and so the second part of this work will discuss the effect that wind forcing has on the coastal freshwater along Cape Cod. Thus, the central question addressed by this work is how does the freshwater east of Cape Cod vary on time scales ranging from several months (seasonal) to a few days (synoptic weather events), and what drives this variability?

Information about the structure and variability of the surface freshwater is obtained using surface data from research ships throughout the Gulf of Maine. This dataset is supplemented by the Finite Volume Coastal Ocean Model (FVCOM, Chen et al. (2003)), which provides a realistic simulation of the circulation and water properties of the Gulf of Maine, and a simple theory for a buoyant plume's response to wind forcing (Lentz (2004) and Moffat and Lentz (2011)). Combined, the data, model, and theory provide insight into the structure and dynamics of the water properties east of Cape Cod on time scales from months to days.

Section 2.2 provides context and motivation for this study. In section 2.3 the seasonal evolution of the coastal freshwater and its response to wind forcing and river freshwater input is discussed using data collected from underway research vessels. In section 2.4, the plume's response to wind forcing on shorter time scales is analyzed using the numerical model and a simple theory. The conclusion section 2.5 synthesizes the results. 


\subsection{Background}

Freshwater is carried to outer Cape Cod via the Western Maine Coastal Current, which flows from Cape Ann, along Cape Cod and into the Great South Channel (see figure 2-1). The portion of this current to the north of Cape Ann has been studied by

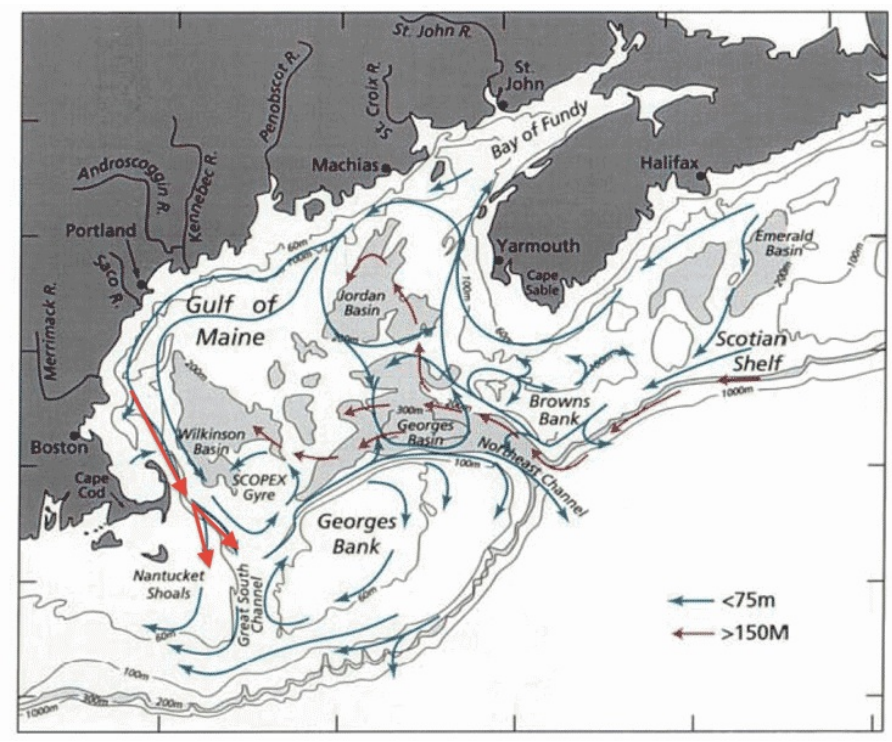

Figure 2-1: Schematic of currents in the Gulf of Maine. Large red arrows indicate the southern extension of the Western Maine Coastal Current (approximately from Cape Ann to the Great South Channel), which carries the surface freshwater to Cape Cod. Figure is adapted from Miller et al. (1998)

Fong et al. (1997) and Fong and Geyer (2001), among others, but the downstream extension along Cape Cod has received less attention (e.g.Chen et al. (1995), Shcherbina and Gawarkiewicz (2008b), Shcherbina and Gawarkiewicz (2008a)). A background flow exists even in the absence of a freshwater plume (Shcherbina and Gawarkiewicz (2008b)), which may be indicative of a larger-scale pressure gradient. Superimposed on this background flow is the surface freshwater, which is the topic of this study. The current and the associated freshwater may play a role in determining the distribution of ecologically and economically important plankton (including Calanus finmarchicus, a key food source for several marine mammals and fisheries species, and Alexandrium fundyense, one of the primary harmful algal bloom species). Additionally, it plays 
a role in nearshore watermass modification and the movement of freshwater and nutrients between the Gulf of Maine and the Middle Atlantic Bight (Shcherbina and Gawarkiewicz, 2008b).

\subsubsection{Seasonal variability}

Chen et al. (1995) provide one of the only accounts of the springtime evolution of the freshwater east of Cape Cod. Therein, the authors suggested that a freshwater plume forms and spreads eastward and becomes fresher from April to June due to seasonal variability of local river discharge, upwelling-favorable wind stress, and large-scale circulation within the Gulf of Maine. These conclusions were largely based upon two hydrographic datasets, consisting of only a few realizations of the surface freshwater spread out over many years. Specifically, a cruise in May-June 1989 found a wider and fresher plume than a similar cruise in April 1988, which the authors attributed to the three-week difference in timing of the cruises relative to the seasonal cycle and a larger river discharge in 1989.

In contrast, two cruises off Cape Cod in spring 2005 separated by one week showed a different pattern: The earlier cruise saw fresher water shallower and farther offshore than the later cruise (see figure 2-2, data from M. Baumgartner). By looking just at these sections, one may reach a very different conclusion from that of Chen et al. (1995) about the seasonality of the surface freshwater east of Cape Cod. Given the nature of these observations, it is necessary to reinvestigate the conclusions about the seasonal cycle of surface freshwater east of Cape Cod using a more comprehensive dataset. The surface underway data analyzed in the following section allows for a comprehensive picture of the seasonality that may be important for copepod aggregation. The first part of this study seeks to test the conclusions of Chen et al. (1995), that the seasonal variability in patterns of freshwater east of Cape Cod is due primarily to riverine input and seasonal wind patterns. 

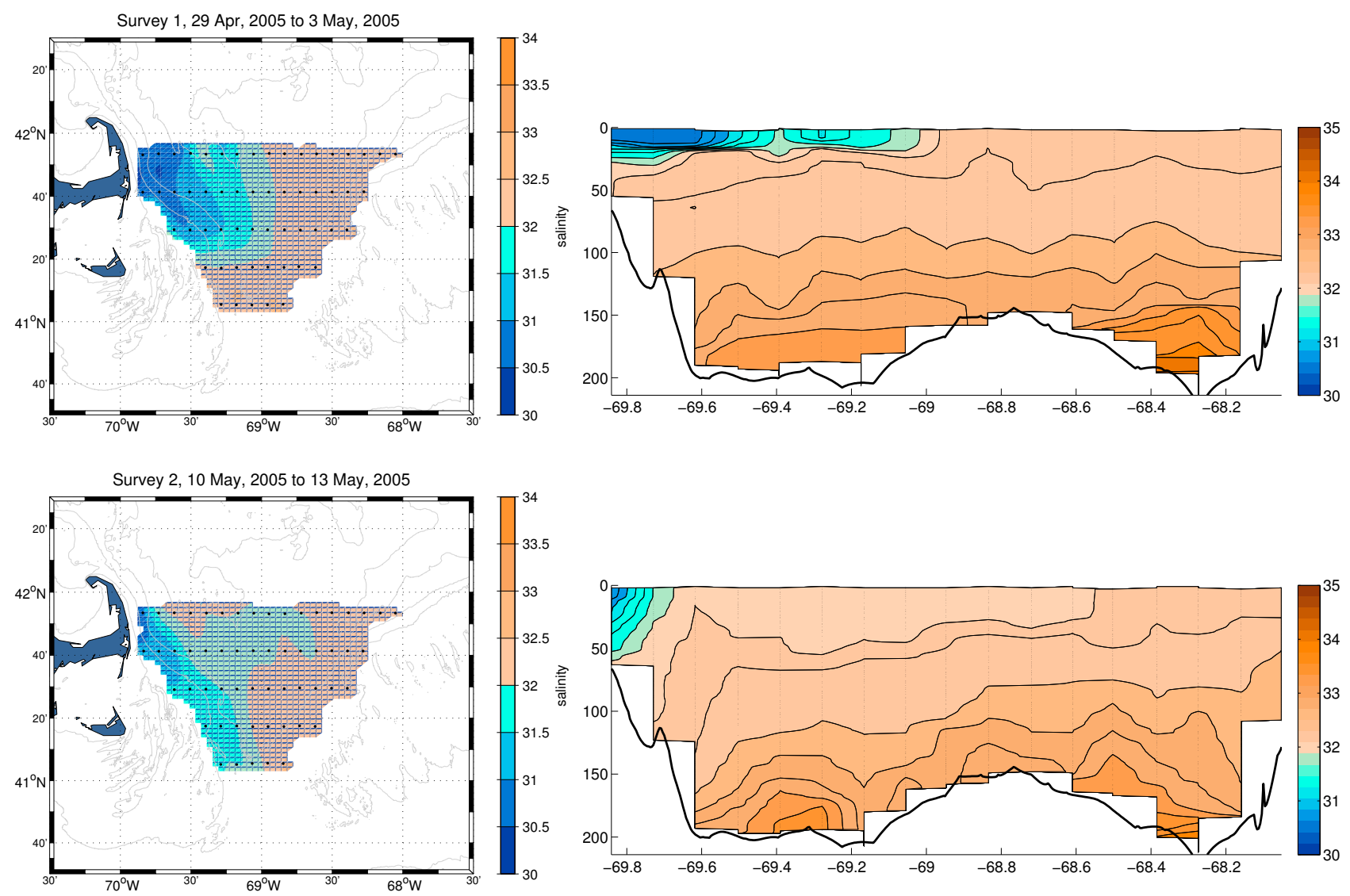

Figure 2-2: Objectively-mapped surface and across-shore CTD sections of salinity before (upper) and after (lower) a large downwelling wind stress event in early May, 2005. Before the storm, the plume is wide, but relatively shallow. After the storm, the plume edge has been advected towards the coast, and the plume reaches much deeper in the water column. Depth sections were taken along upper-most CTD line shown by black dots in left-hand panels. Data from M. Baumgartner, WHOI

\subsubsection{Shorter time scales}

If the single sections of figure 2-2 are ineffectual in describing the seasonal cycle, they do exhibit variations in the surface freshwater on shorter time scales which may also be important to copepod aggregation. In between the two sections, a large storm transited the area. During the storm, strong downwelling winds persisted for a few days, advecting the freshwater at the surface towards the coast. The response of a buoyant plume to wind forcing, as shown by Lentz and Largier (2006) among others, is conceptually simple: A downwelling (positive) wind stress will make the plume narrower, while an upwelling (negative) wind stress will make the plume wider. 
Fong and Geyer (2001), Lentz (2004) and Moffat and Lentz (2011) have developed companion theories for the response of an alongshore-uniform buoyant coastal plume to upwelling and downwelling wind-forcing, respectively, which has been shown to perform well in predicting characteristics of some coastal plumes. If the theories are able to predict the structure of the surface freshwater in this region, and if copepod aggregations form and persist near the edge of the surface freshwater, then the theories may provide a means to predict the cross-shore location of copepod aggregations.

A form of the theory for the response of buoyant plumes to along-shore wind forcing similar to Lentz (2004) and Moffat and Lentz (2011) is reviewed and compared to the numerical model in section 2.4. The derivation will largely follow the other works, but a few key points will be emphasized. First, in the previous applications of this theory, the absolute geometry of the plume is predicted given an initial plume state before the onset of wind forcing; the theory here is used instead to predict changes in the plume characteristics that were compared to changes observed in the model. Secondly, versions of the theory that allow for different levels of entrainment of interior salty fluid into the fresh plume are presented here: one with no entrainment, one with entrainment only due to deepening of the wind-mixed layer, and one with continuous entrainment. This will indicate which modes of entrainment are important for this system. Finally, the previous work is strictly two-dimensional; it assumes a constant flux of freshwater from some distance far upstream. A version that attempts to compensate for variation in the upstream conditions is included. In all, the following will provide four separate estimates of the plume characteristics, which can be compared to each other and the realistic model to help elucidate the plume's physical controls on daily to weekly time scales.

These theories were applied mostly to regions where a classical buoyant plume with a steady supply of freshwater impinges on the continental shelf and propagates down the coast of its own accord. East of Cape Cod, however, is hardly a classical buoyant plume: There is the aforementioned background flow independent of the freshwater supply, as well as some of the most vigorous tides in the world, a highly 
variable supply of freshwater, bottom slope and coastline, as well as two pathways for freshwater to reach this location (one along Stellwagen Bank, the other from Cape Cod Bay). Here, the theories are adapted to apply to the region to evaluate their predictive skill in light of the complicating factors.

\subsection{Seasonal cycle of coastal freshwater}

\subsubsection{Shipboard TSG Dataset}

The dataset described herein includes surface salinity and temperature measurements made from moving research vessels. Many research vessels are equipped with a thermosalinograph (TSG), which measures surface temperature and salinity while the vessel is underway. The Oceanography Branch of the NOAA Northeast Fisheries Science Center archives TSG data from each of its cruises throughout the Gulf of Maine. This data may be accessed online through a database found at www.nefsc.noaa.gov. Additionally, regional surveys aboard the R/V Oceanus as a part of the Gulf Of Maine TOXicity program (GOMTOX) were conducted throughout the Gulf of Maine, and

are included with the NOAA data. Surface data from CTD casts archived by the National Oceanographic Data Center are included as well. In all, the data spans the years from 2000 to 2010, and includes more than 100 different research cruises. As shown in figure $2-3$, the data covers a large portion of the region defined by $71-68^{\circ} \mathrm{W}$ and $41-43^{\circ} \mathrm{N}$. However, the data is not uniformly dense in time, but rather reflects a significant seasonal bias (also in figure 2-3).

\subsubsection{Seasonal evolution of the coastal freshwater}

Chen et al. (1995) surmised that the surface properties east of Cape Cod are a result of river discharge and seasonal wind patterns. To test this hypothesis, wind and river discharge data were compared to surface properties to ascertain the sources of variability. 


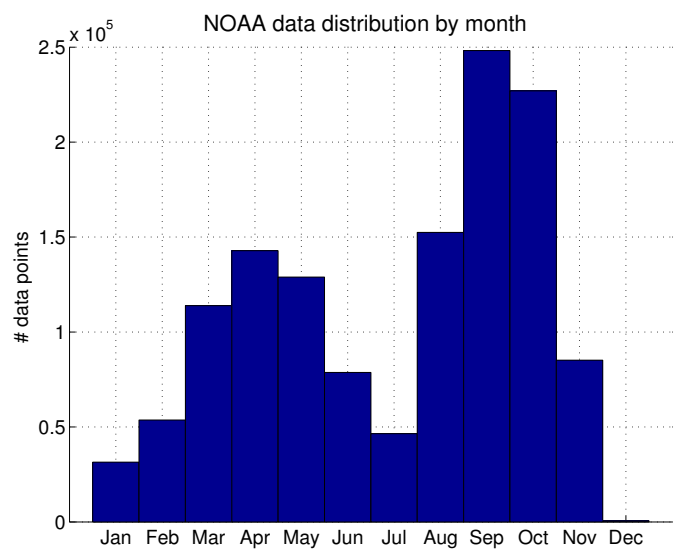

(a) Histogram showing data distribution by month

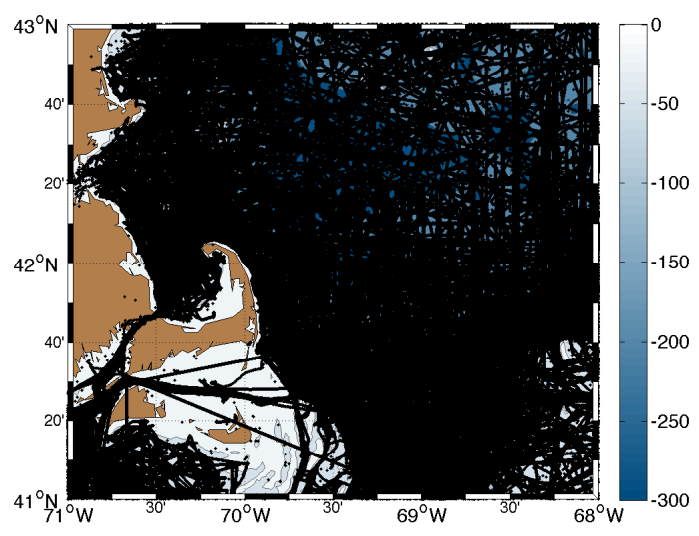

(b) Map of all surface data points

Figure 2-3: Data distribution in time $(a)$ and space $(b)$ for the shipboard underway data

\section{Surface properties from shipboard dataset}

The surface shipboard underway data was box averaged in 5 along-coast bins (see figure 2-4). All the data in each box was averaged by month to create a time series of temperature and salinity in each box (shown in figure 2-5). The shape of these bins is chosen to follow the local bathymetry and to include data along the approximate pathway of the coastal current (as shown in figure 2-1). We chose to use bins defined by the local bathymetry to account for along-coast variability in the bathymetry.

To create a composite yearly cycle, data in each box were averaged by day of the year (shown in figure 2-6). The composite yearly averages clearly show the seasonal cycle of temperature and salinity. In temperature, the cycle repeats itself almost exactly from year to year mostly in response to the seasonal cycle in surface heat flux. In contrast, there is more variability in the salinity pattern, both in an interannual and alongshore sense. In all boxes, surface salinity is lowest in early summer. This signal is largest in the northern boxes, and is almost non-existent in the box farthest south (box 1).

In order to elucidate some of the different modes of variability, empirical orthogonal functions (EOFs) were computed. As one might expect, almost all (96\%) of the 


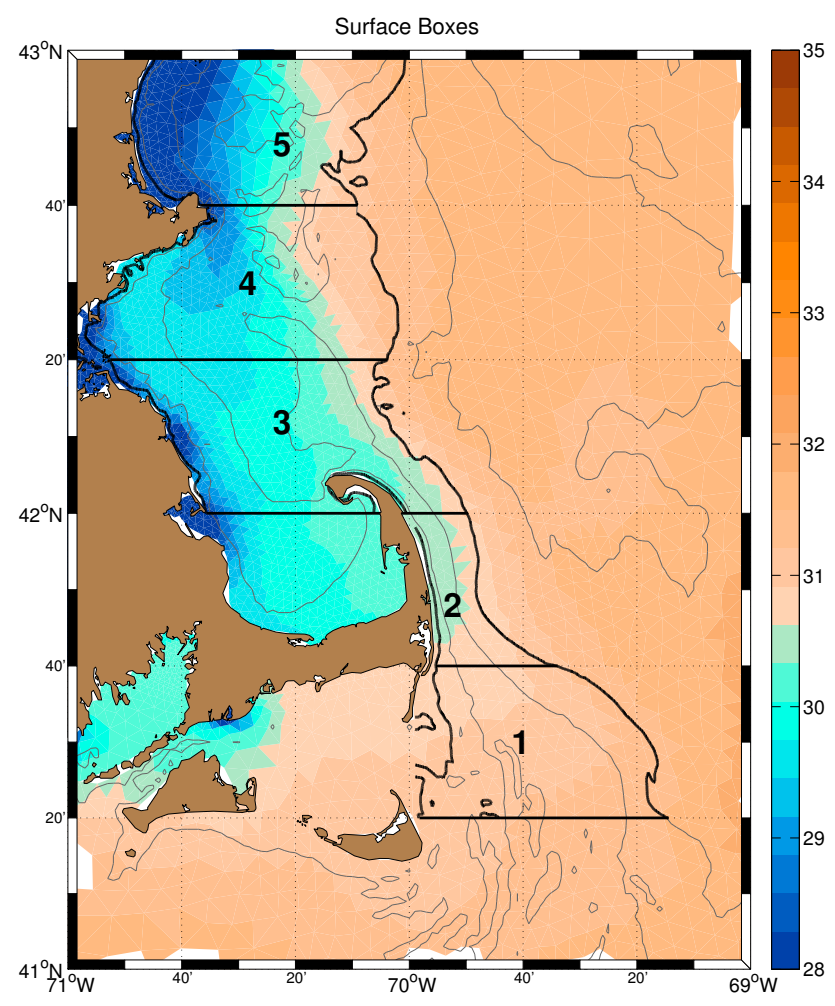

Figure 2-4: Bins for box averaged data. Each bin extends $\frac{1}{3}^{\circ}$ in latitude, and is bounded inshore by the $10 \mathrm{~m}$ isobath and offshore by the $100 \mathrm{~m}$ isobath. Boxes are numbered from box 1 (farthest south) to box 5 (farthest north). Background color is a snapshot of surface salinity from a numerical model (FVCOM).

variance in the temperature time series is contained in the first EOF, which represents the seasonal cycle in sea surface temperature. The magnitude of this mode is slightly larger in the southern boxes than in the north, but mostly the strength of the seasonal cycle is equivalent in all boxes.

The first EOF of salinity, as in the case of temperature, includes part of the seasonal cycle. The cycle for salinity will reach a minimum in the late spring after the spring freshet, and will peak in the late fall/early winter. This mode contains approximately $83 \%$ of the variance in the salinity time series. It appears that this mode also contains some of the inter-annual variability, including the anomalously fresh spring of 2005 (Anderson et al. (2005)). The structure function of this mode (figure 2-7, panel $b$, upper) shows that the magnitude is largest in the north (box 5) 


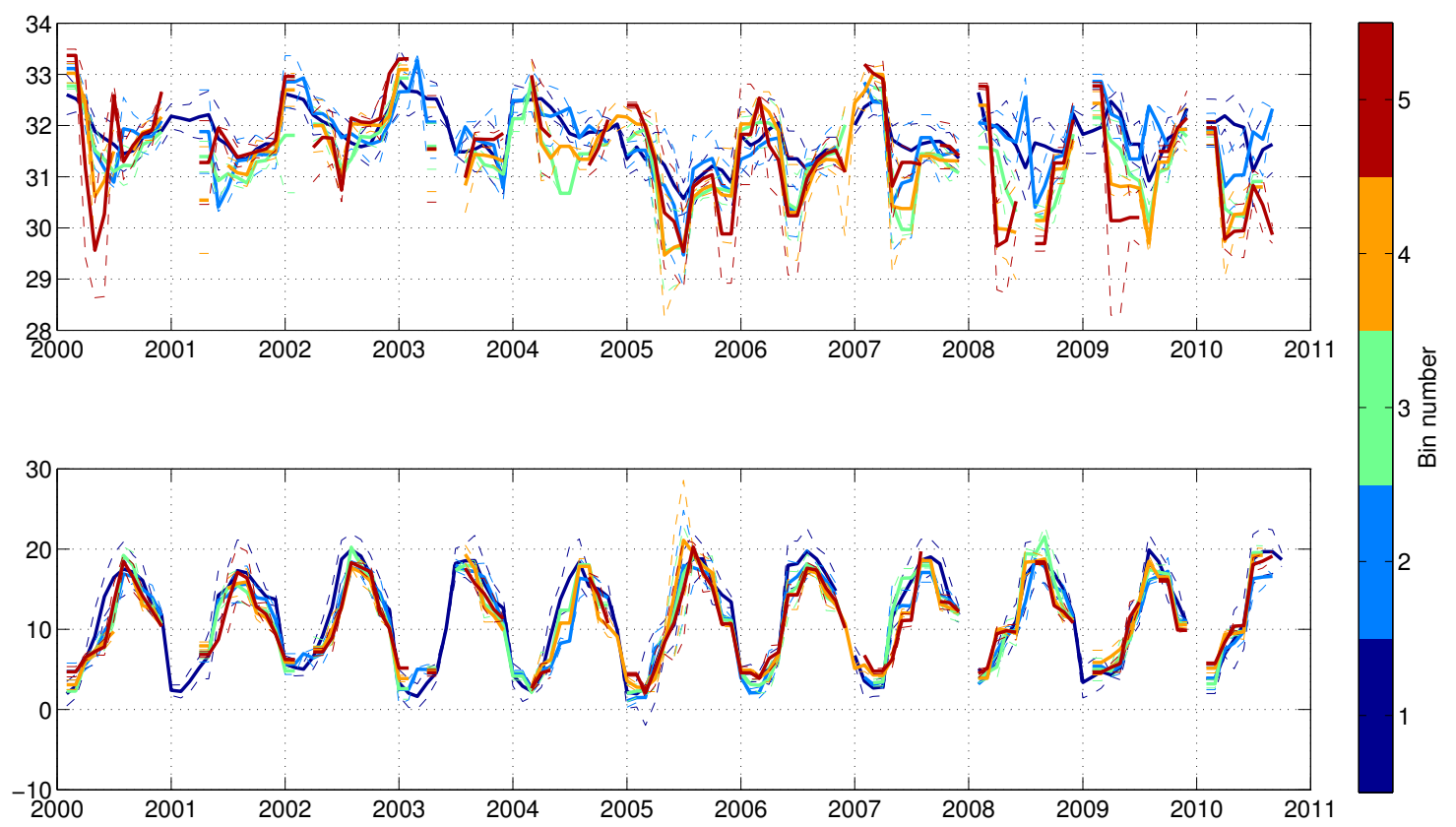

Figure 2-5: Box-averaged monthly salinity (upper panel) and temperature (lower panel) for the 5 boxes pictured in figure 2-4. Dashed lines indicate standard deviation of the data averaged within this box.

and weakest in the south (box 1). This is consistent with the notion that the seasonal cycle has the largest amplitude in the north, closer to the river sources.

The second EOF of salinity contains close to $10 \%$ of the variance. The timeseries function contains some seasonal variability as well, but slightly shifted in time to peak in mid summer (especially in later years of the record). This mode may represent the effect of seasonal changes in wind forcing, however more data is required.

\section{Surface forcing: river discharge and winds}

Hetland and Signell (2005) compiled the river discharge in cubic meters per second for five major Gulf of Maine rivers (Penobscot, Kennebec, Androscoggin, Saco, and Merrimack); this will be used in conjunction with the surface winds at the NDBC buoy in Massachusetts Bay (NDBC buoy \#44018) to analyze the effect of river discharge and wind forcing on the surface freshwater in the lower Gulf of Maine. The river discharge data were taken from USGS streamflow gauges in each river. The timing 

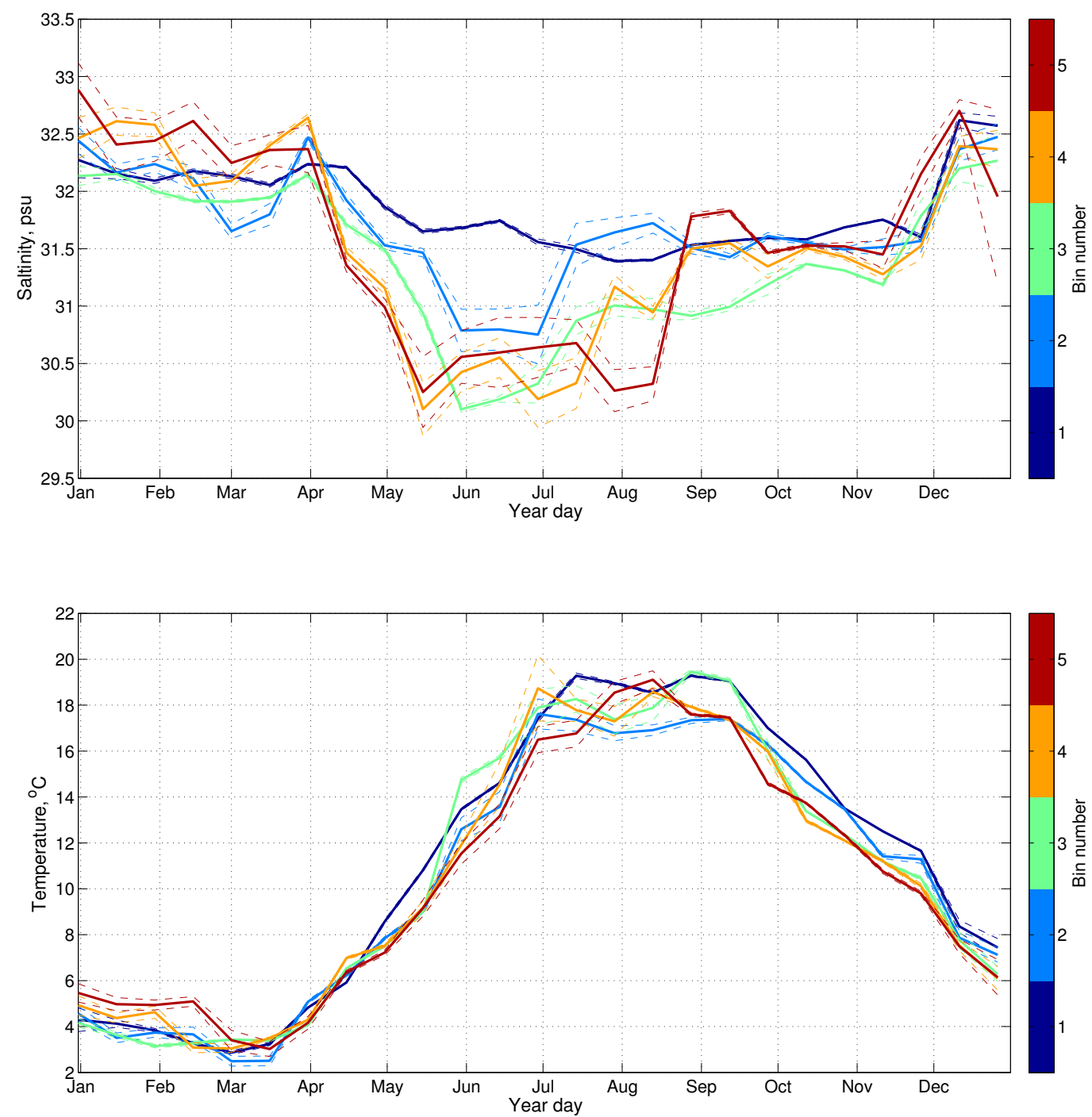

Figure 2-6: Yearly composite surface salinity (upper) and temperature (lower) for the 5 boxes in figure 2-4. Data were bin-averaged by yearday (15 day bins). Dashed lines show standard deviation of the binned data.

and difference in relative magnitude of the river discharge is fairly consistent between rivers. Here, the wind speed and direction have been smoothed with a 30 hour boxcar filter to remove the daily cycles. To visualize the seasonal cycle in wind forcing and riverine discharge, a composite yearly average is formed by averaging all data by day of the year, which is shown in figure 2-8. For the river discharge, all rivers are first summed together, then averaged.

The seasonal cycle of riverine discharge is such that every spring there is a large 

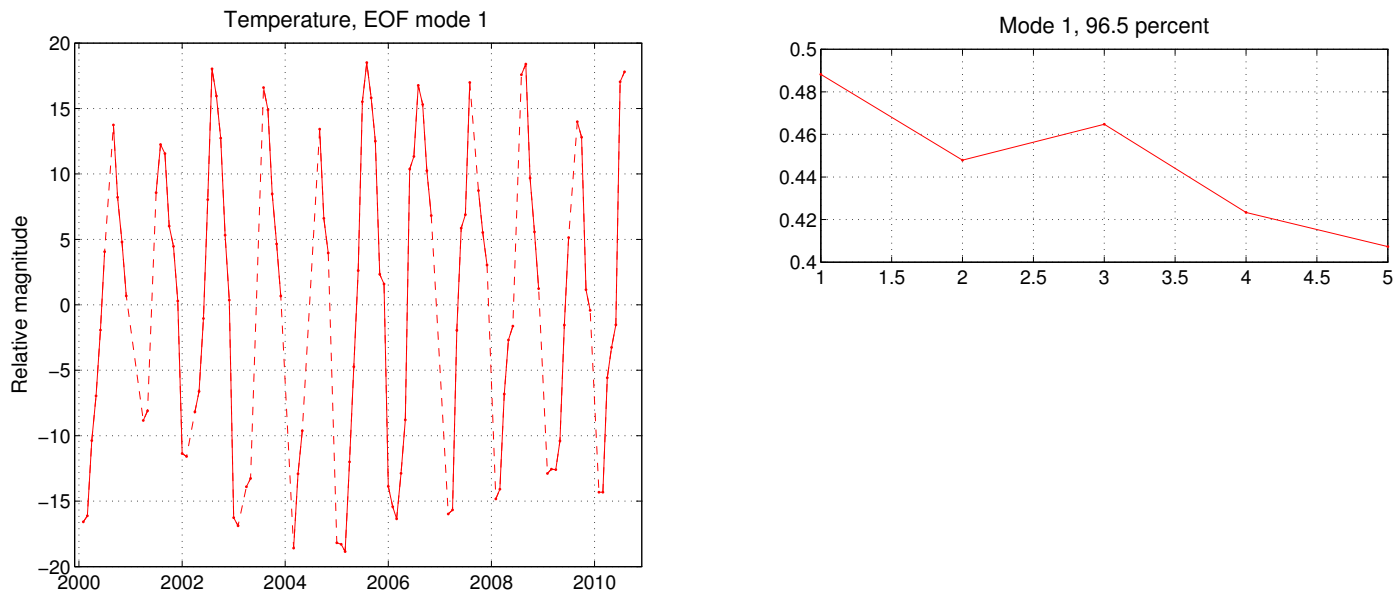

(a) First EOF of temperature
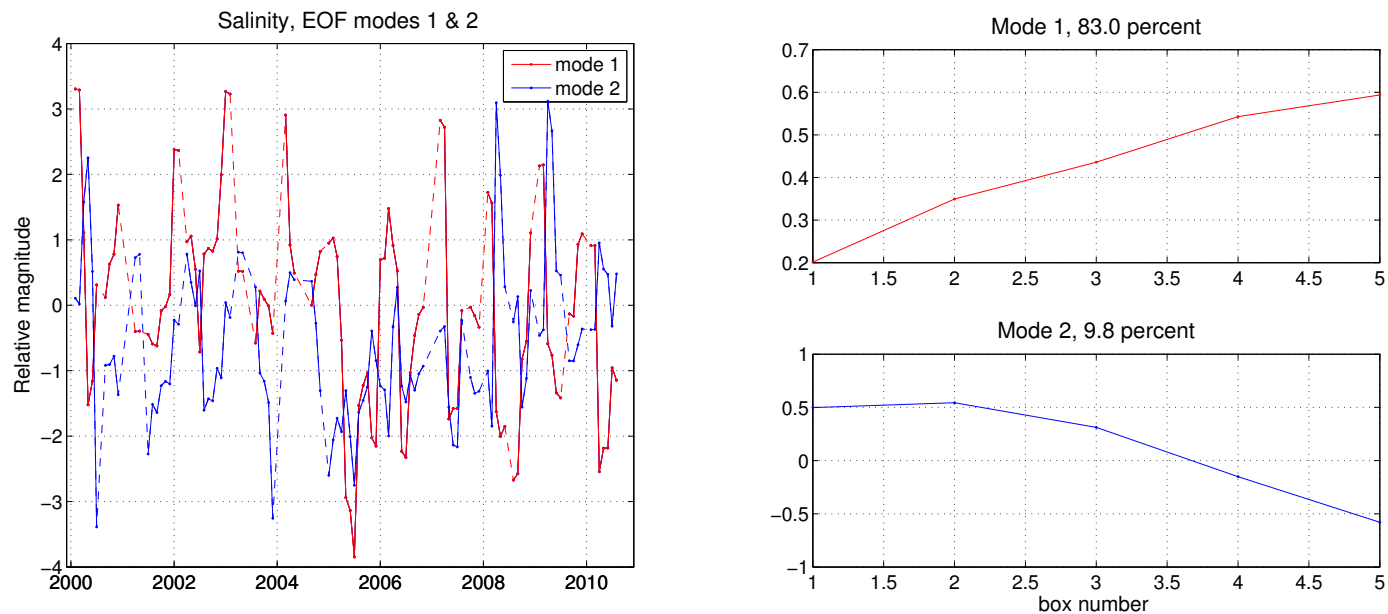

(b) First two EOFs of salinity

Figure 2-7: Empirical orthogonal functions of temperature $(a)$ and salinity $(b)$. Lefthand panels are time series, right-hand panels represent structure of each EOF as a function of box number. Box number 5 is the northern-most box (near Cape Ann), while box 1 is the southern-most (in the Great South Channel, see figure 2-4). Dashed lines in time series connect points separated by missing values, while solid lines connect all adjacent points. Percentage of variance contained in each mode is shown in the title of the right-hand panels

freshet event, which inputs a significant amount of freshwater to the coastal ocean. This event occurs at some point in April or early May. The river discharge decreases throughout the summer, and is followed by a slight increase in the late fall/early winter. The surface winds in winter are relatively strong and either in the cross-shore 

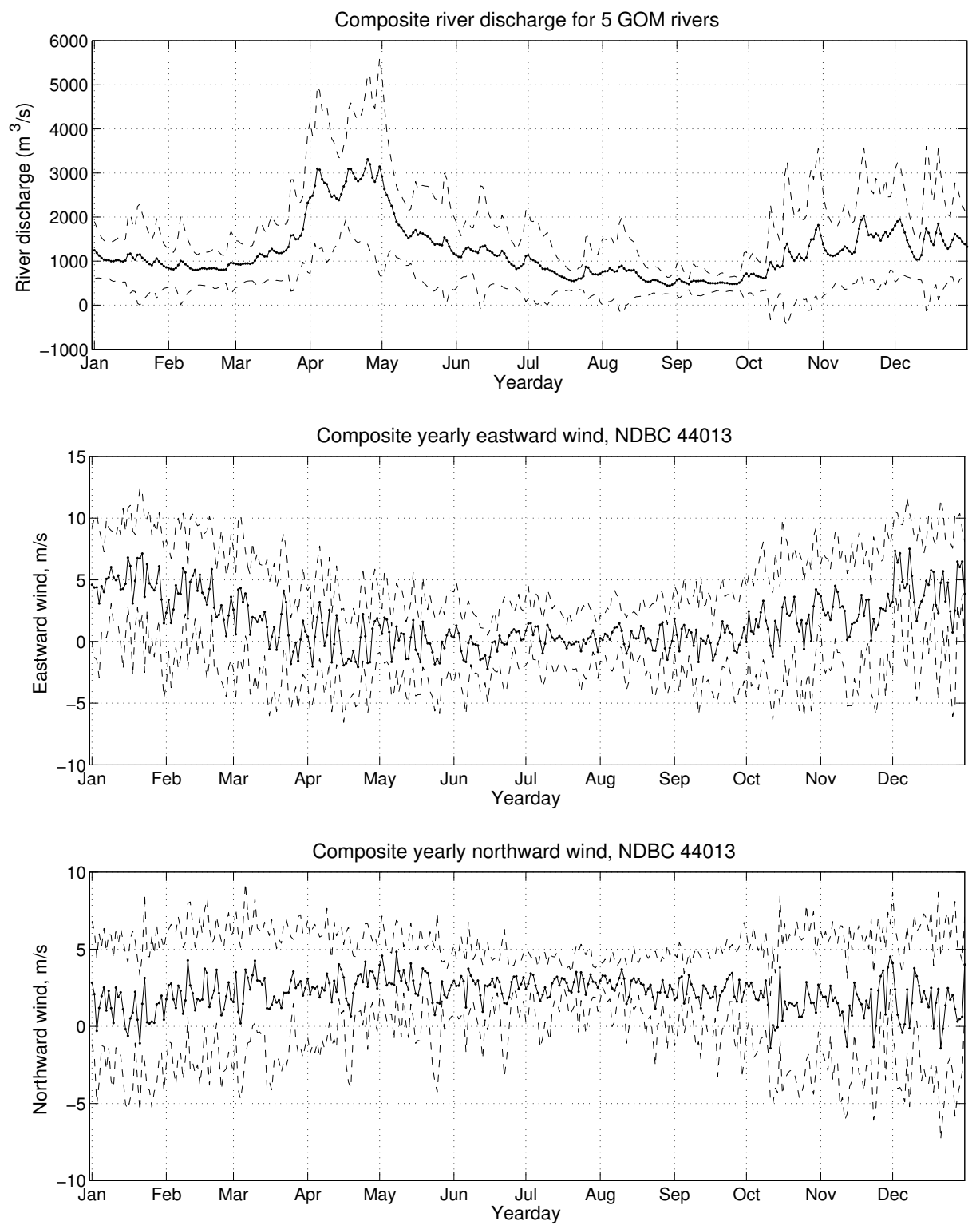

Figure 2-8: Yearly average river discharge sum for the five major GOM rivers (top), eastward wind (middle), and northward wind (bottom) at NDBC buoy 44013. Dashed lines indicate \pm 1 standard deviation about the daily mean.

direction or downwelling-favorable (northwesterly), while the winds in summer are generally weaker and upwelling-favorable (southwesterly). The springtime transition from downwelling to upwelling happens in roughly March or April. Of course, there are significant departures from these mean cycles on time scales ranging from daily to interannual. For example, river discharge varies significantly between years: In April-May 2005 it was much larger than a typical year (more than twice as large). 
Also, synoptic wind events lasting for a few days can lead to wind speeds much faster than the average, and can be in the entirely opposite direction. The shorter time-scale variability will be tackled in a subsequent section.

Figure 2-9 shows the composite average river discharge plotted versus the composite average eastward and northward wind speed. There is a weak, but statistically significant, correlation between the wind speed and river discharge $(0.14, \mathrm{p}$-value $=$ 0.004), indicating that stronger winds tend to coincide with higher discharge rates. This is not likely a causal relationship, but rather reflects the fact that there are seasonal cycles in both the wind and river discharge, resulting in a correlation. Neither the direction of the wind nor the eastward or northward wind components exhibits a statistically significant relationship to the river discharge rate (figure 2-9).

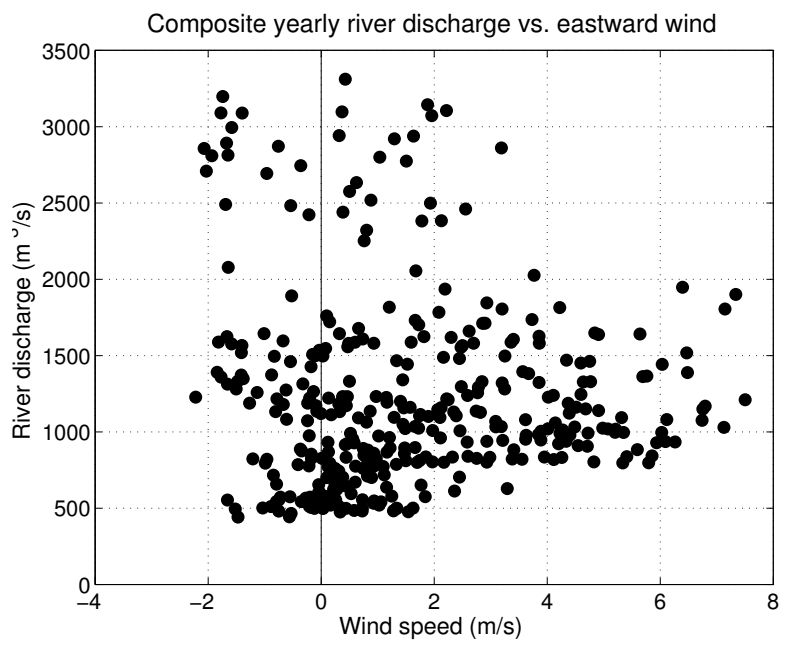

(a) Eastward wind speed

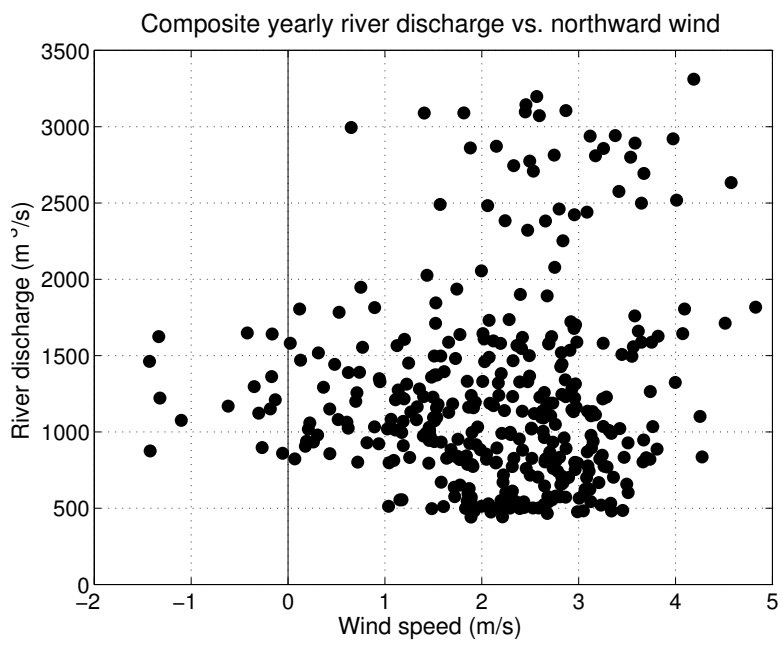

(b) Northward wind speed

Figure 2-9: River discharge plotted versus eastward (left) and northward (right) wind.

\subsubsection{River discharge vs. observed sea surface salinity}

Seasonal cycles of river discharge and salinity in each box are shown in figure 2-10. The salinity in each box decreases in spring in response to enhanced river discharge. The correlation between the seasonal cycles of river discharge and surface salinity is -0.4 ; as river discharge increases in the spring, the coastal waters become more 


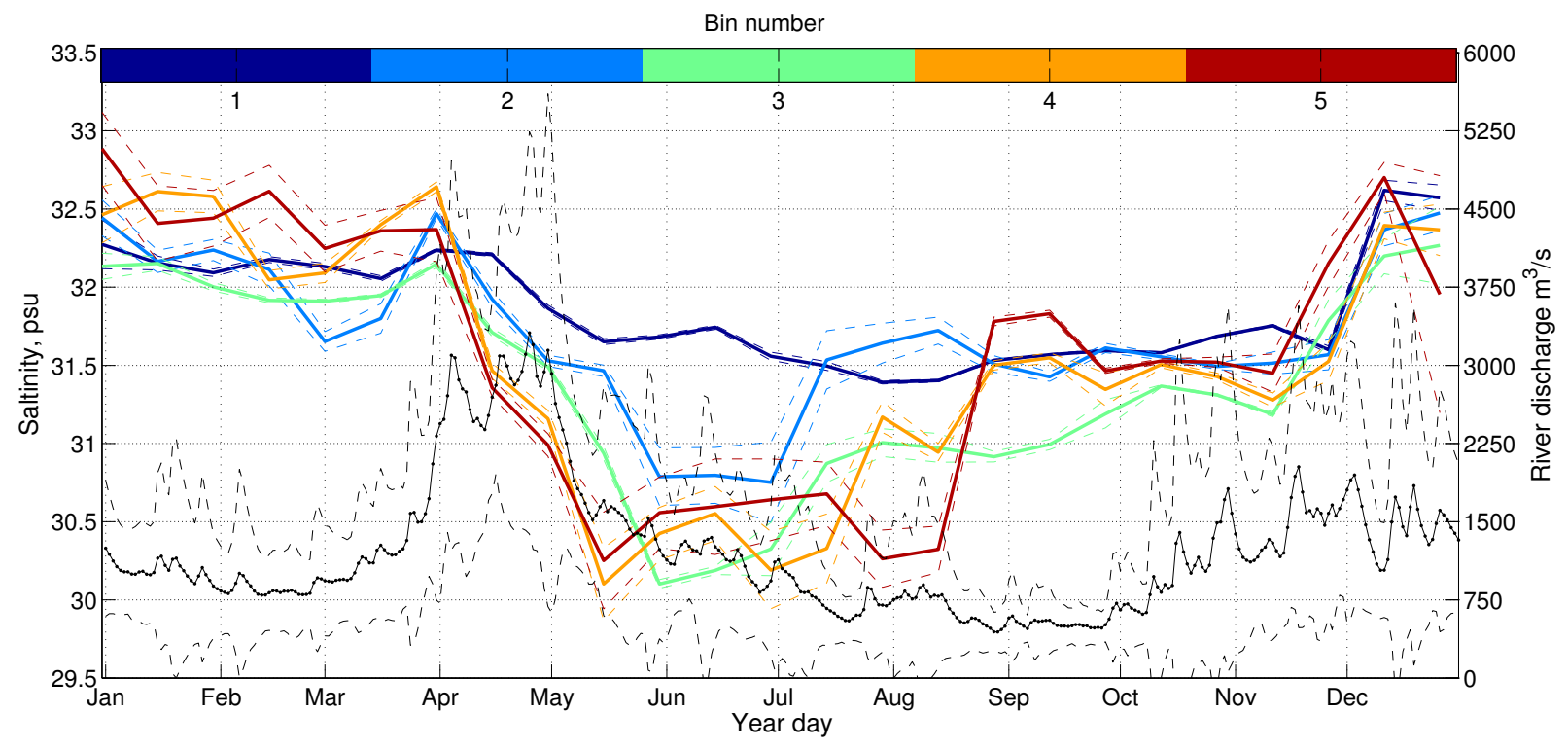

Figure 2-10: Seasonal river discharge versus surface salinity (bin averaged to 15 days). Correlation coefficient between the two time series is -0.4 (p-value $<0.005)$.

fresh and salinity decreases. We have not quantified here the role of Scotian shelf waters, which have their own seasonal cycle (fresher in the spring, saltier in the fall). There is a slight increase in the river discharge in the fall which does not correspond to a decrease in surface salinity in the coastal waters. This could be due to the Scotian shelf water (upstream of the GOM rivers) becoming more salty during this time (competing against the effect of the increased river discharge during this time), but we are unable to resolve this increase with the data in hand.

The data in figure 2-10 indicates a plausible relationship between the river discharge and salinity east of Cape Cod, however it is not clear that the relationship is causal; both time series contain an intense seasonal cycle that may or may not be related. A better test for causality would be to compare the interannual variability. Figure 2-11 shows the monthly mean salinity in box 2 versus the cumulative river discharge from the five major rivers in figure 2-8 with the seasonal cycles removed. The correlation between the two time series suggests that the surface salinity at Cape Cod is due in part to riverine input to the Gulf of Maine, with larger river discharges corresponding to lower surface salinities. The fact that the correlation is stronger 


\begin{tabular}{|c|c|c|c|c|}
\hline Box $\mathbf{5}$ & Box 4 & Box $\mathbf{3}$ & Box $\mathbf{2}$ & Box $\mathbf{1}$ \\
\hline$-0.55(35.5$ days $)$ & $-0.56(26.5$ days $)$ & $-0.48(36$ days $)$ & $-0.38(41.5$ days $)$ & $-0.40(43.5$ days $)$ \\
\hline
\end{tabular}

Table 2.1: Correlation (lag) between 15-day average salinity and river discharge for each of the 5 boxes.

between the two time series when the rivers are lagged forward in time suggests that the salinity signal takes some time to propagate from the riverine sources to box 2 .

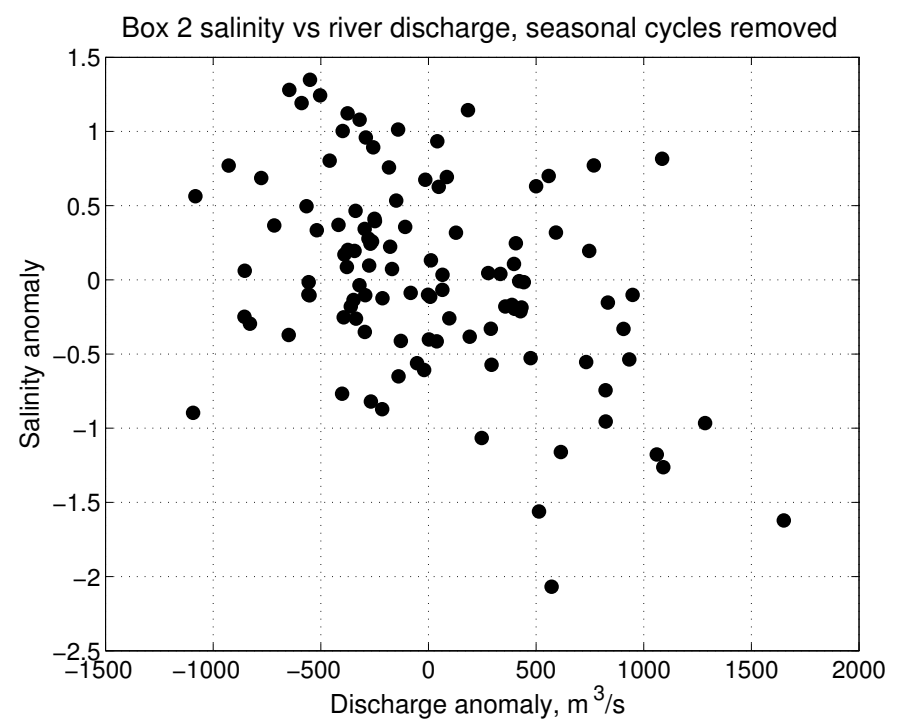

Figure 2-11: Monthly river discharge anomaly plotted versus monthly surface salinity in box 2, with seasonal cycles removed. Monthly river discharge has been forwardlagged in time by approximately 40 days (the lag that gives maximum correlation to salinity anomaly). Correlation coefficient $=-0.42$ (p-value $<<0.005)$

There are no significant rivers injecting freshwater close to Cape Cod, so if the seasonal variation in freshwater at Cape Cod is due to Gulf of Maine rivers, then the freshwater must propagate to the Cape from the upstream sources. Thus, one might expect that the surface salinity in the northern boxes would "see" the effect of the large spring freshet first, while the boxes farther downstream would lag behind those upstream by a factor consistent with the advective time between the boxes. Qualitatively, one may notice such a pattern in figure 2-6, where it appears that the surface salinity in box 5 begins to decrease earliest in the spring, followed shortly thereafter by the other boxes. 
Using surface drifters Manning et al. (2009) estimated the average advective time from Cape Ann to Cape Cod to be approximately 13 days, with a standard deviation of 8.1 days, corresponding to an advective speed of about $14 \mathrm{~cm} / \mathrm{s}$. Figure $2-12$ shows the lagged correlation between shipboard surface salinity in box 5 and box 2 for an average spring. The lag at maximum correlation for these two time series is 11.1 days, and the distance separating the boxes is approximately $100 \mathrm{~km}$, corresponding to an advective speed qualitatively similar to that observed by Manning et al. (2009). Table 2.2 shows the lags between each of the 5 boxes. Mostly, the farther separated the boxes are in space, the larger the lag between them, again suggesting propagation of the surface signal alongshore over the progression of the season. This suggests that the surface salinity off Cape Cod is advected with the coastal current flow from upstream freshwater sources in the Gulf of Maine.
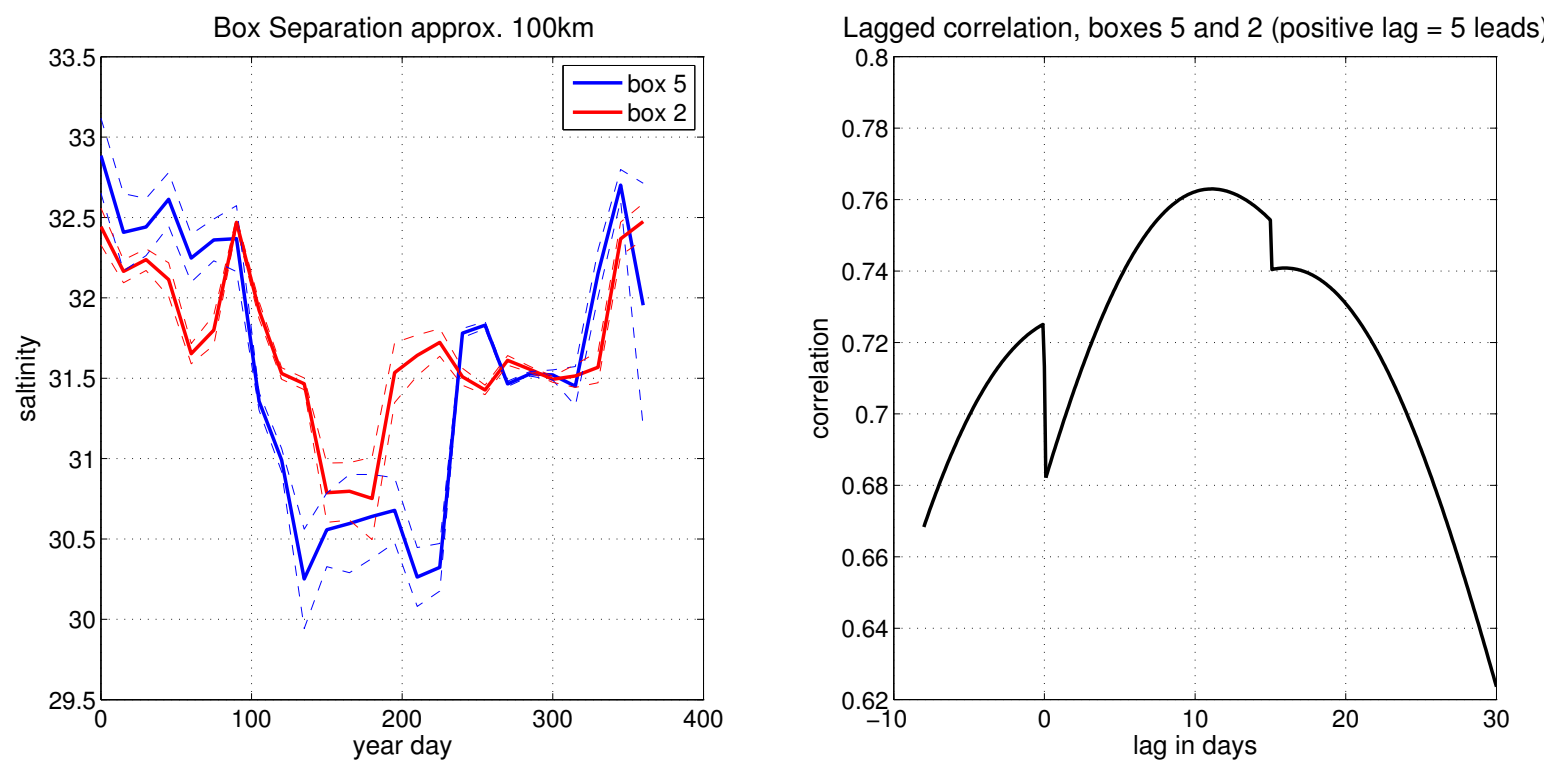

Figure 2-12: Time series (left) and lagged correlation (right) between 15-day average surface salinity in box 5 and box 2. Positive lag, box 5 leads. 


\begin{tabular}{|l|c|c|c|c|c|}
\hline & Box 5 & Box 4 & Box 3 & Box 2 & Box 1 \\
\hline Box 5 & - & $4.8(.92)$ & $10.1(.90)$ & $11.1(.76)$ & $12.6(.80)$ \\
\hline Box 4 & $-4.8(.92)$ & - & $6(.92)$ & $5.2(.84)$ & $7.6(.76)$ \\
\hline Box 3 & $-10.1(.90)$ & $-6(.92)$ & - & 0 & $8.8(.80)$ \\
\hline Box 2 & $-11.1(.76)$ & $-5.2(.84)$ & 0 & - & $4.5(.74)$ \\
\hline Box 1 & $-12.6(.80)$ & $-7.6(.76)$ & $-8.8(.80)$ & $-4.5(.74)$ & - \\
\hline
\end{tabular}

Table 2.2: Lags (in days) between 15-day average salinity in each box. Number in parentheses is correlation at maximum lag.

\subsubsection{Wind forcing vs. observed sea surface salinity}

Does the wind forcing play a role in determining the observed patterns in sea surface salinity? Figure 2-13 shows the seasonal wind speed at NDBC buoy 44018 as well as the average salinity in the 5 boxes. The wind speed is weaker in the summertime when the surface is fresher, and stronger in the winter when the surface waters are saltier. As a result, there is good correlation between the wind speed and surface salinity. The wind direction also has a seasonal cycle (northwesterly in winter, southwesterly in the summer), but this seasonality is small compared to the high degree of variability of the wind direction on shorter time scales (particularly synoptic storms). The fact that wind speed and surface salinity are correlated could be due to the effect of mechanical mixing by the wind: strong wind in winter mixes the coastal freshwater with saltier interior water, thus driving surface salinity upward. In summer, when the wind relaxes, the coastal waters become more fresh. This relationship, however, is likely not the leading cause of the seasonal cycle in coastal freshwater.

On longer time scales, interannual variability in the wind speed and direction are difficult to discern given the level of high-frequency variability in both time series. On shorter time scales (namely, that of synoptic storms), however, the winds may be very important in determining the structure of coastal freshwater. We explore this possibility in the following section. 

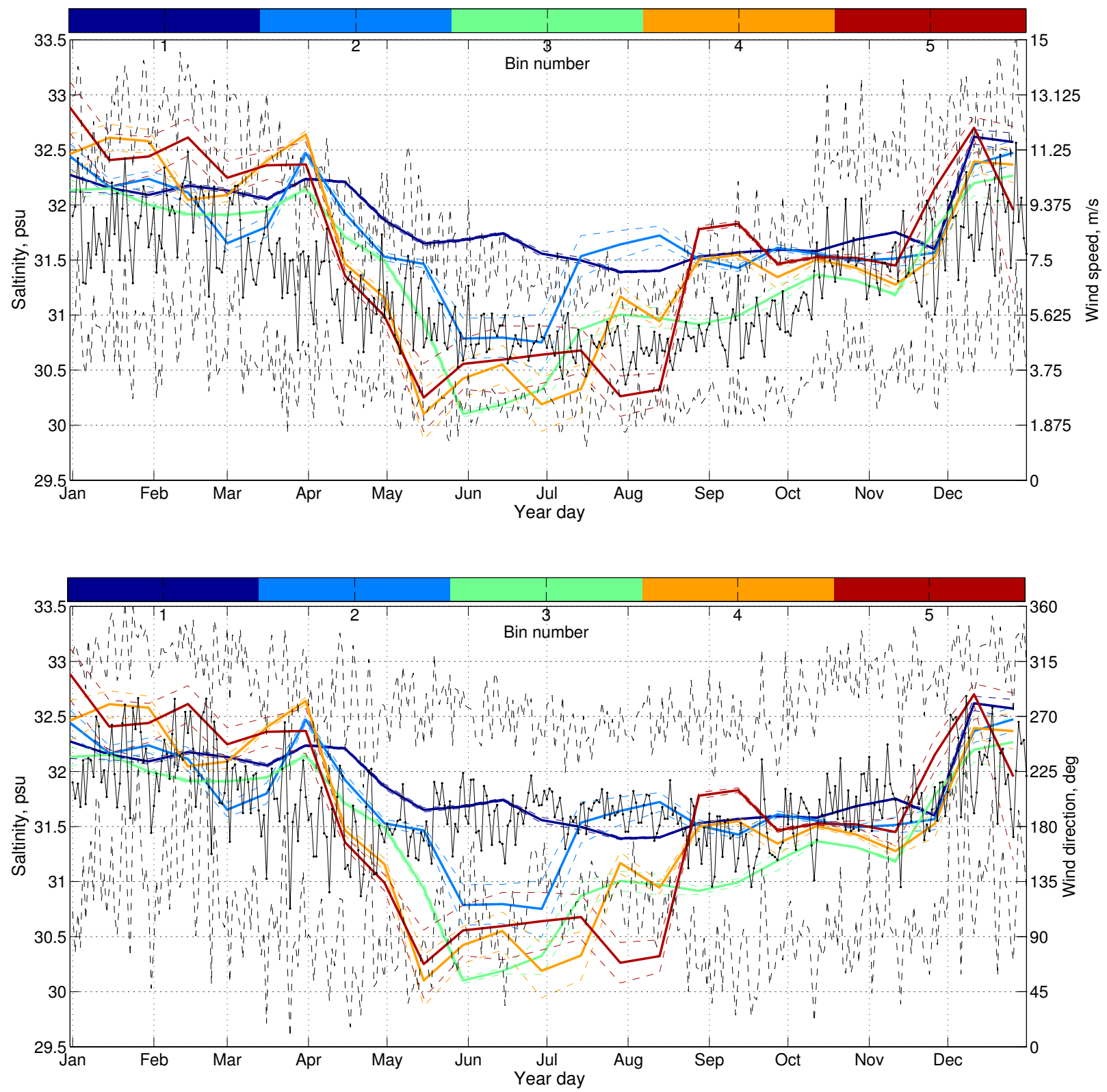

Figure 2-13: Seasonal wind speed and direction versus surface salinity (bin averaged to 15 days). Wind speed is strongly correlated to the surface salinity $(\mathrm{r}=0.75)$. Seasonal cycle in wind direction not well determined (large uncertainties due to variable wind direction).

\subsubsection{The salinity front and its response to wind forcing and river discharge}

In order to analyze the effect of wind forcing and river discharge on the structure of the surface freshwater, it is necessary to develop a metric to identify the edge of the 
coastal freshwater. From 2000 to 2010, there were more than 100 different research cruises in the Southern Gulf of Maine region. When a vessel travels from inshore waters offshore, it crosses the region that may contain the freshwater pool. In all, there are more that 220 such instances, from here on called "crossings", in the dataset. It is possible to use the cross-shelf salinity distribution in each of these crossings for the location of the edge of the pool of freshwater by identifying the position of the intense across-shore salinity gradient (the salinity front). This analysis is described here.

The across-shore sections are extracted by visually identifying periods in the data when a vessel was moving approximately east-west. Most of the crossings chosen are those in which the vessel is transiting to some point far enough offshore such that any reasonable coastal freshwater, if it exists, will be entirely transited by the vessel. The extracted data are binned zonally in $1 \mathrm{~km}$ sized bins, then smoothed with a running-mean filter to reduce noise. In this study, the edge of the freshwater pool is identified by a relatively intense salinity gradient separating fresher water near the coast from saltier water offshore. First, the location and magnitude of the maximum salinity gradient is determined from the smoothed salinity section. Next, the maximum salinity gradient is compared to the average salinity gradient across the entire crossing. If the maximum gradient is larger than five times the average, and the salinity inshore of the location of the maximum gradient is smaller than the salinity offshore, then the crossing is retained for analysis; otherwise, it is discarded. The location of the salinity front is taken to be the location of the maximum salinity gradient. Finally, the crossing is grouped into one of five possible locations: Nantucket Sound, Cape Cod, Race Point, Stellwagan Bank, and Cape Ann, depending roughly on the latitude of the crossing (determined by visual inspection). Of the 228 crossings in the dataset, 77 contained a useable observation of the salinity front based upon the above criteria. An example of such a crossing is shown in figure 2-14. For each salinity front the position of the front, the magnitude of the salinity gradient at the front ("frontal strength"), and the salinity anomaly (salinity difference between 
inshore and offshore of front) are compared to river discharge and winds.

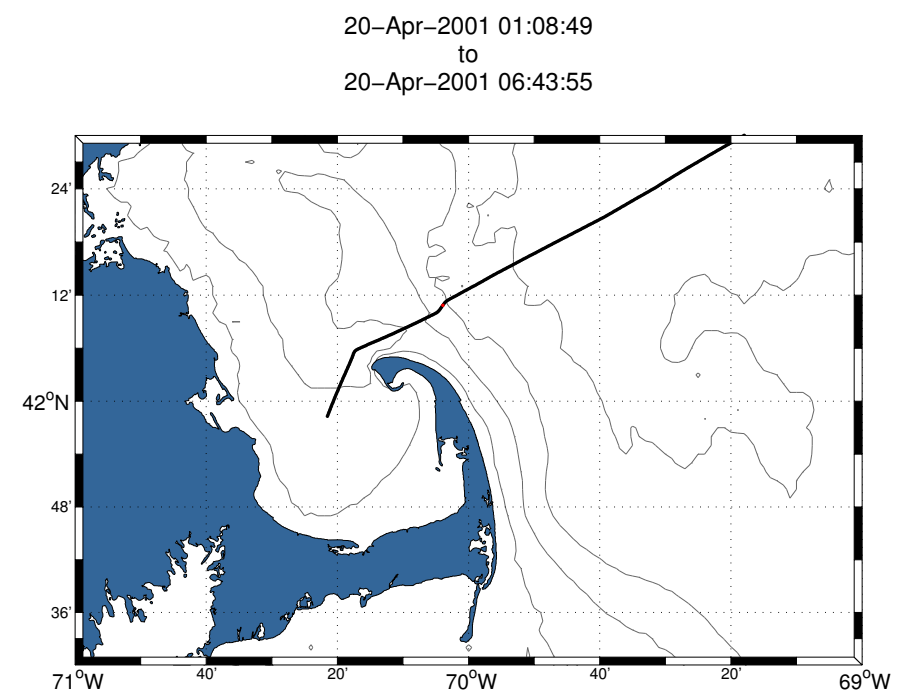

(a) Map of crossing

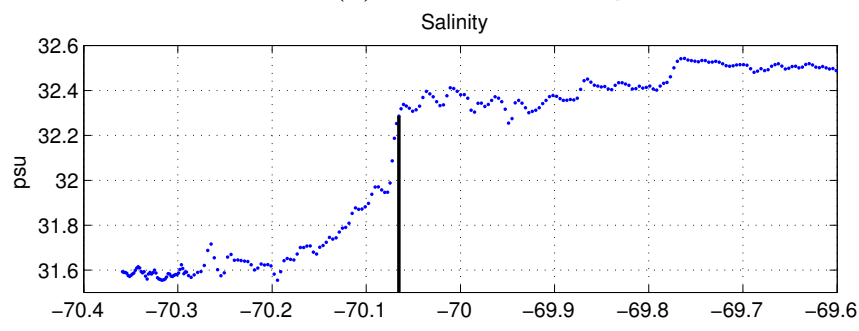

(b) Salinity vs longitude

Figure 2-14: A crossing of the coastal freshwater in April, 2001 from the R/V Albatross IV containing a salinity front. Surface salinity (bottom) measured by thermosalinograph while exiting Cape Cod Bay and traveling to the west across the southern edge of Stellwagan Bank. The measured salinity increased as the ship crossed the surface freshwater pool. The position of the salinity front, indicated by the black line, is found using the method described.

After the analysis was completed, the distance from the $20 \mathrm{~m}$ isobath to the salinity front (this isobath was chosen because it closely follows the coastline but is slightly smoother, allowing the calculation to be made easily), the salinity gradient at the front, and the salinity anomaly between the water inshore and offshore of the front were found for each of the 77 realizations.

Figure 2-15 depicts a histogram of the number of the 77 observations by month normalized by the number of crossings in that month. The histogram was normalized to account for a seasonal sampling bias (see figure 2-3). Even though this figure 
attempts to normalize to account for sampling effort in terms of number of crossings, no effort has been made to account for the relative quality of the crossing. This dataset represents an amalgamation of different cruises, each with unique scientific objectives, which may make the crossing during that particular cruise more or less likely to contain a good observation. Also, the quality of the TSG data collected on these individual cruises may vary. Without the ability to account for the objectives of individual cruises, however, figure 2-15 indicates that one is slightly more likely to observe a salinity front in summer or fall than in winter or spring (possibly due to enhanced mixing during seasons characterized by stronger winds).

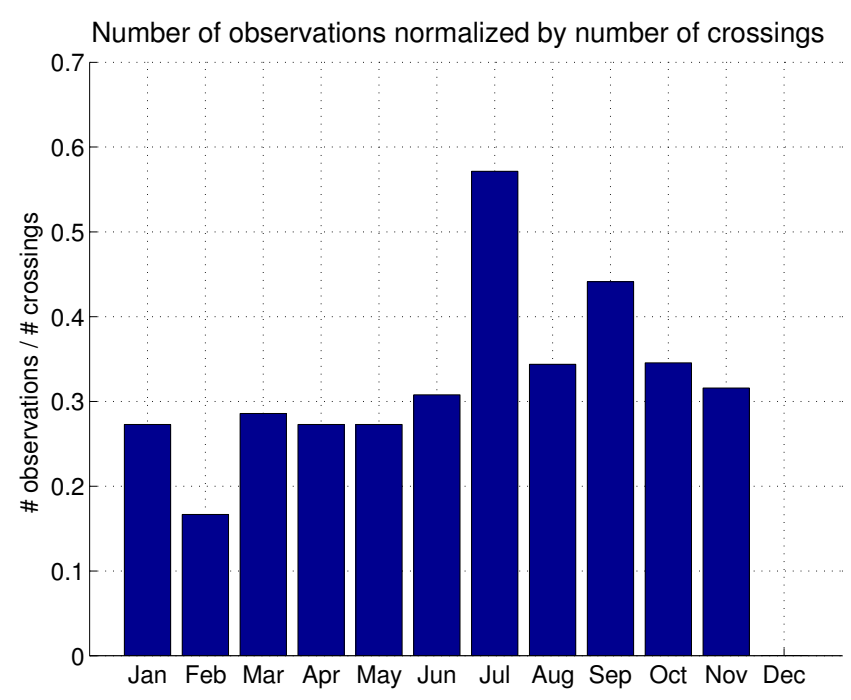

Figure 2-15: Histogram of number of coastal freshwater observations by month, normalized by the number of crossings.

Figure 2-16 shows each of the three characteristics as a function of month. The distance to the front is smallest in winter (if it exists at all), grows throughout the spring, and is maximum in summer (June-August). The front intensity and salinity anomaly are maximum during the spring, specifically the months of April and May.

The intensity and salinity anomaly appear to respond in an intuitive manner to the river discharge. The relatively intense fronts observed in April and May are likely due to their proximity in time to the spring freshet. As the rivers discharge high volumes of freshwater, relatively intense fronts with high salinity anomalies (corresponding to fresher water inshore of the front) form. After the spring freshet, the salinity front 

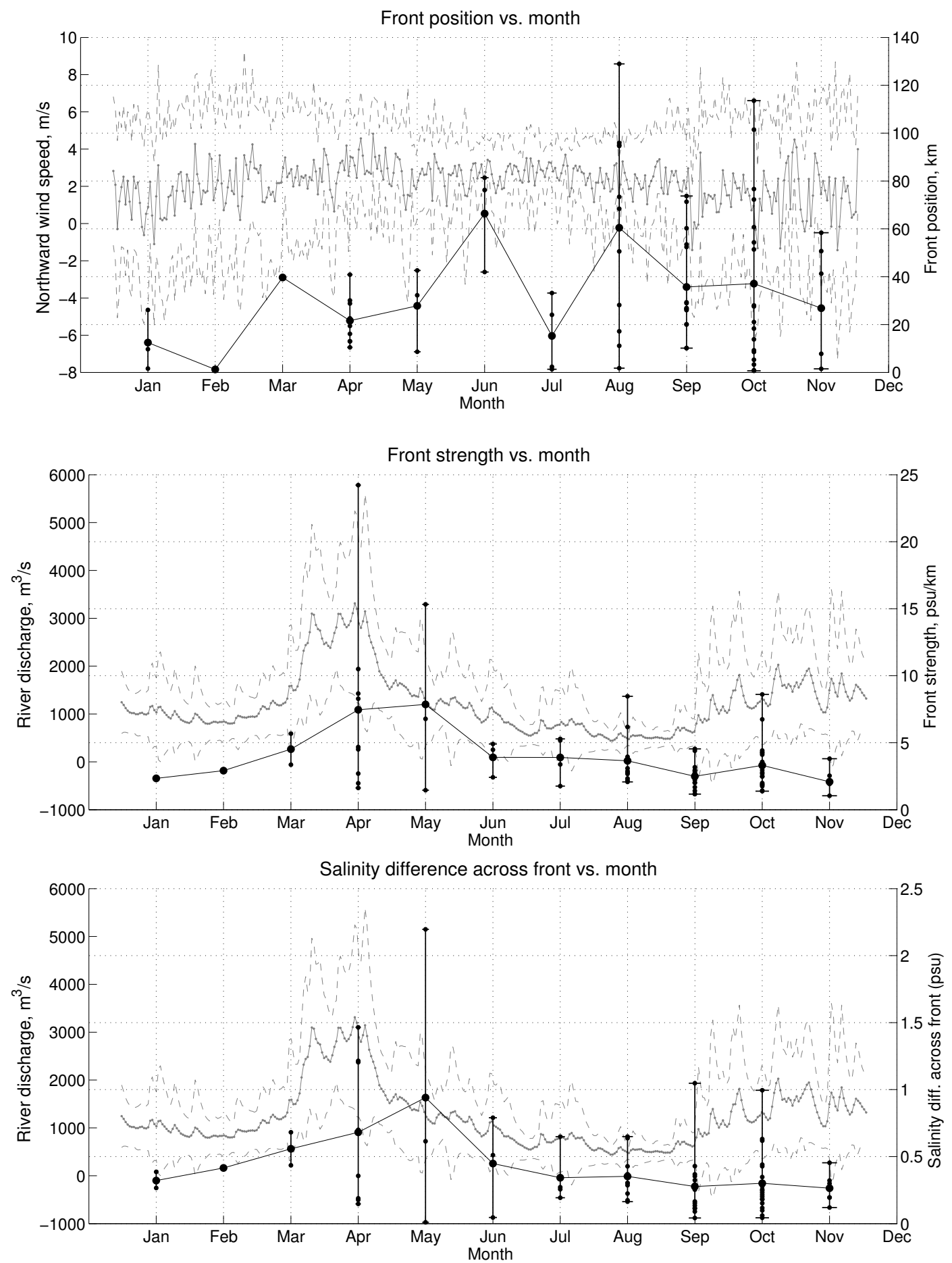

Figure 2-16: Monthly averages of distance from 20m isobath to front (upper), front strength (middle), and plume salinity anomaly (bottom) versus month. Small dots are 77 individual crossings, large dots show monthly mean, while bars indicate range over that month. In grey, the seasonal cycles of northward wind (upper) and river discharge (middle and lower) are plotted versus yearday with \pm 1 standard deviation. 
intensity will progressively weaken as the freshwater is mixed with interior waters.

Indeed, figure 2-16 shows weaker plumes in the summer and fall, when river discharge is lower.

Downwelling winds in winter advect the front onshore, while upwelling winds in summer advect the front offshore, in a manner consistent with Ekman theory. Thus, one might expect to find the front further offshore at the end of the upwelling season (early fall). It is difficult to tell that this is the case in figure 2-16, however the position of the front may also vary on shorter time scales (see, for example, figure $2-2)$.

\subsubsection{Direct wind forcing of the plume}

To evaluate the effect that winds have on shorter time scales, the 77 plume observations were compared to instantaneous along-shore wind forcing to assess the impact local winds have on the front position. Two meteorological buoys (NDBC 44013 in Massachusetts Bay and 44018 in the northern Great South Channel) were used to estimate the along-shore and across-shore components of the wind (using whichever buoy was closest to the front). Winds were again smoothed with a 30-hour filter, and wind stress was computed following Large and Pond (1981), and the wind stress was rotated $90^{\circ}$ to approximately align with the bathymetry; thus, a positive wind stress corresponds to downweling-favorable conditions.

Figure 2-17 shows front position as a function of both alongshore and across-shore wind stress. These data suggest that the salinity front off Cape Cod is moderately sensitive to synoptic wind forcing. The correlation coefficient between the alongshore winds and front position is -0.35 , and in general downwelling winds correspond to front positions closer to shore while upwelling winds correspond to front positions farther offshore. This is qualitatively consistent with observations of other coastal freshwater systems (see, for example, Lentz and Largier (2006)). 

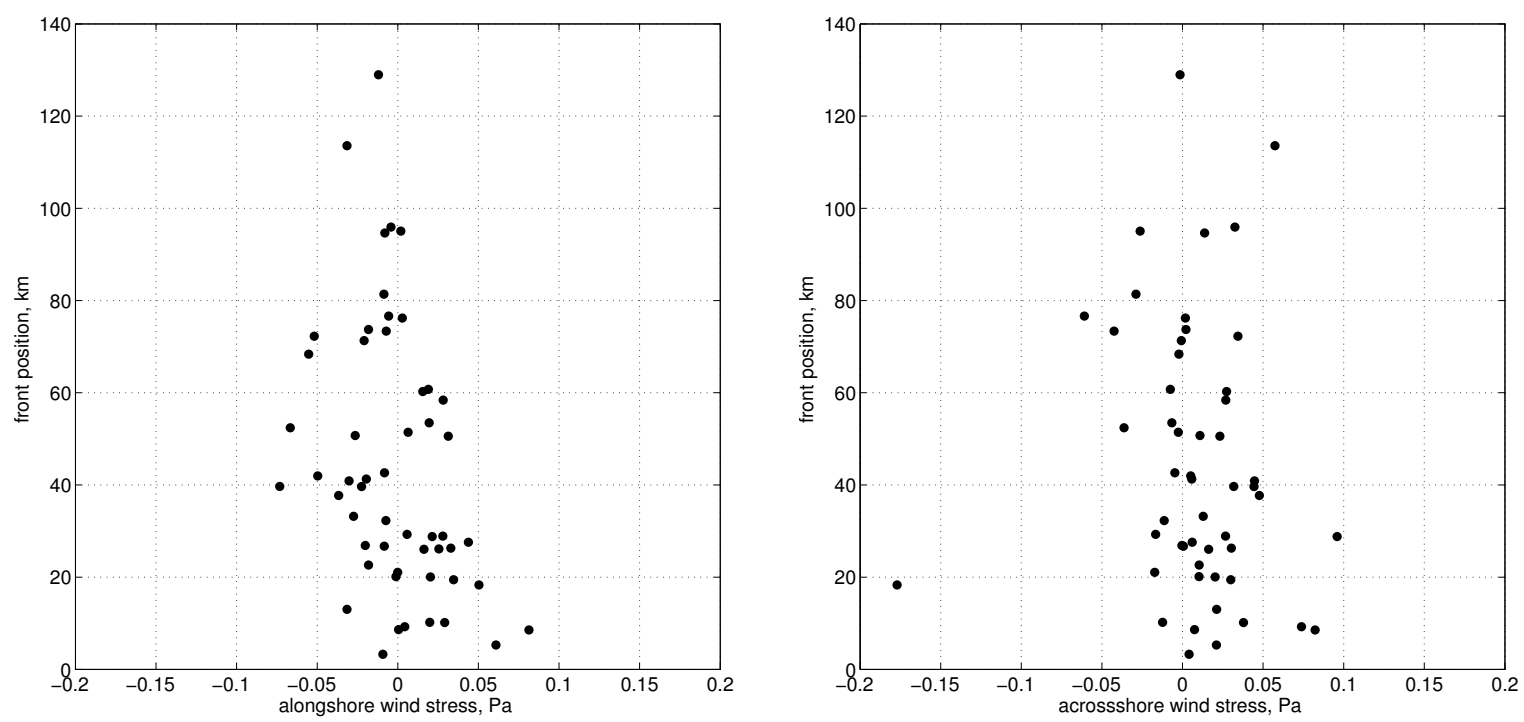

(a) Alongshore wind. Positive is southward (down-(b) Across-shore wind. Positive is offshore, negative welling), negative is northward (upweling) is onshore

Figure 2-17: Front position (km) vs alongshore (left) and across-shore (right) wind stress $(\mathrm{Pa})$. Correlation coefficients are $-0.35(\mathrm{p}$-value $=0.01)$ and $-0.14(\mathrm{p}$-value $=0.32)$ for the alongshore and across-shore winds, respectively.

\subsection{Realistic Numerical Model of the Plume and Wind Forcing Theory}

Since the freshwater front appears to respond in an intuitive manner to along-shore wind forcing, it is possible that one may be able to predict certain characteristics of the freshwater structure using a simple theory. There are three primary goals for this section: First, after describing the model in section 2.4.1, the model's skill will be evaluated against the underway TSG dataset (section 2.4.2). Second, theory for the response of a buoyant plume to along-shore wind forcing will be developed to apply specifically to the Cape Cod freshwater system (section 2.4.3). In section 2.4.4, the structure of the freshwater in the model will be compared with theoretical estimates to analyze the theory's skill. 


\subsubsection{Model Description}

Simulations using the FVCOM model are used in this analysis. This unstructuredgrid, finite-volume, free-surface primitive equations model has a variable horizontal grid resolution that ranges from $1 \mathrm{~km}$ in the coastal waters to $10 \mathrm{~km}$ offshore. Vertically, the model has 31 sigma-levels. Closure is achieved via the Mellor-Yamada 2.5 and Smagorinsky turbulence closure schemes for vertical and horizontal turbulence, respectively. The model's 1 hour time step resolves tidal motions well, and three years of data (2005-2007) are used in this analysis.

The high spatial resolution near the coast makes this model well-suited for studying the freshwater plume. A hydrographic section from the model starting near Nauset Harbor extending offshore 130km (pictured in figure 2-18) is used in the following analysis. At every model time step, plume width, depth, cross-sectional area, and density anomaly are identified. Collectively, these plume characteristics will be compared to the simple theory derived in a subsequent section.

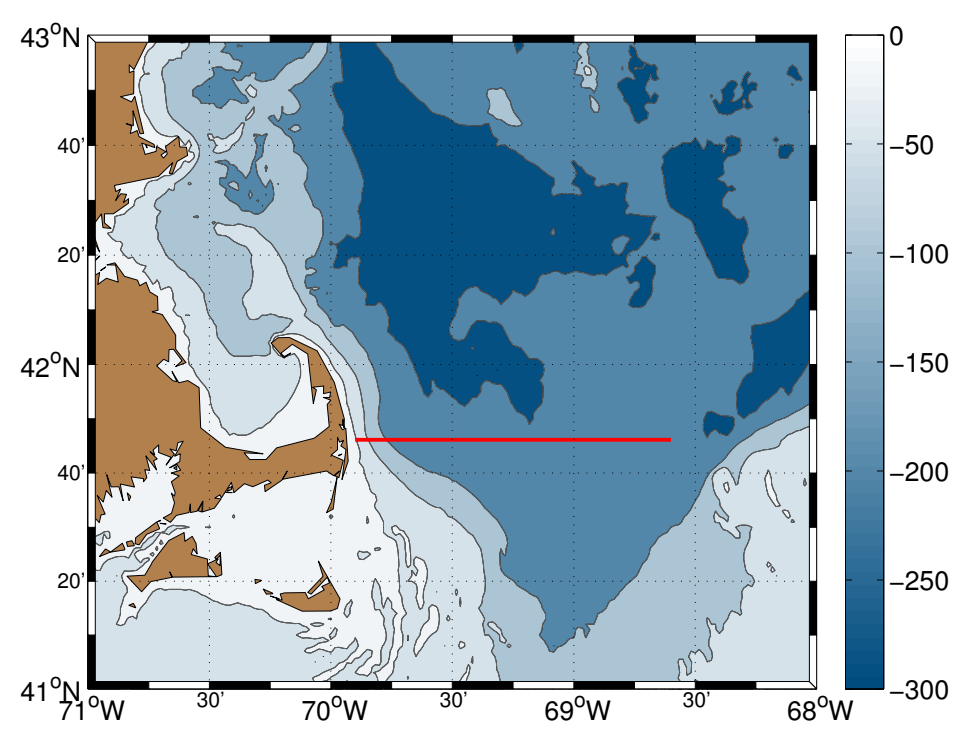

Figure 2-18: Analysis is performed using model hydrographic data taken along a section extending from approximately Nauset, MA, offshore 130 kilometers. 


\subsubsection{Comparison between model and surface dataset}

Since the model contains information at a much higher spatial and temporal resolution covering the entire spring, it is better suited to analyze the effect of wind forcing on the plume in question. However, without some indication that the model captures the variability seen in the data, any conclusions made about wind forcing would be tenuous at best. In section 2.3.2, the seasonal cycle of salinity was analyzed by binaveraging surface data. To validate the model for use in representing the coastal waters, a similar analysis is performed here and compared to the results of section 2.3.2. The model's uppermost layer and the surface TSG data are bin-averaged in identical boxes in order to see how the model compares. The model years 2005 through 2007 are compared with surface TSG data over the same period. The first two EOFs are computed for the model and data, and are plotted together in figure $2-19$.
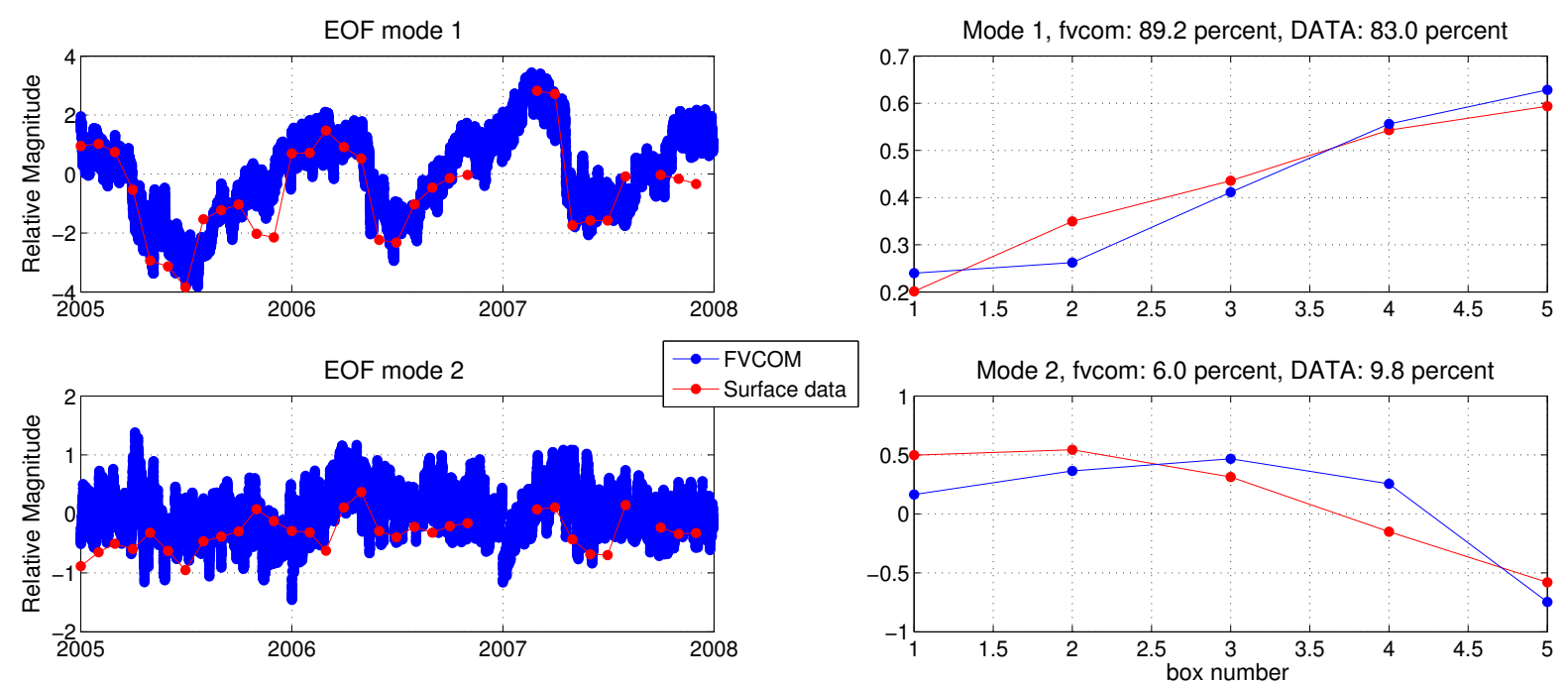

Figure 2-19: First two empirical orthogonal functions of surface salinity in 5 surface boxes in southwestern Gulf of Maine. Red lines correspond to surface salinity data, while blue lines represent the FVCOM surface salinity. Left-hand panels are time series, right-hand panels represent structure of each EOF as a function of box number. Variance contained in each mode is shown in titles

EOF mode one, the component containing $89 \%$ and $83 \%$ of the variance in $\mathrm{FV}$ - 
COM and the data, respectively, shows the decline of the salinity along the coast over the course of the spring. The structure and time series functions of this mode are very similar: both appear to essentially contain the springtime freshening of the coastal waters, with the magnitude of the seasonal cycle larger farther north. The time series, structure, and percentage of variance explained of the second EOF mode in the model also appears to agree with the data.

Plotted in figure 2-20 is the position of the salinity front at the Nauset line (figure 2-18) versus along- and across-shore wind stress for the model (black dots) and observations (red dots). For completeness, the depth at which the frontal isohaline intersects the bottom (the so-called "plume depth") is shown versus the same winds. As in the observations, the front position is related to the along-shore wind forcing consistent with the simple conceptual understanding: Upwelling (negative) wind stress yields fronts farther offshore, while downwelling (positive) wind stress yields fronts closer to shore.

In other coastal systems it has been shown that upwelling winds make the plume shallower, while downwelling winds make the plume deeper, which appears to loosely be the case here. In spring time in the southwestern GOM, there does not appear to be much of a relationship between the across-shore winds and the plume depth. To better understand the response to wind forcing, a simple theory is developed in the following section to compare to the model. In the following, four versions of the theory will be presented. These four forms allow for three different levels of entrainment of interior water into the plume and one version that includes a parameterization for the upstream conditions (termed the "no entrainment", "some entrainment", 'full entrainment", and "upstream" versions).

\subsubsection{Theory for wind forcing of buoyant plume}

The theory developed below is designed to describe a buoyant coastal plume. While the coastal freshwater described above has some of the characteristics of a buoyant plume, one may argue that it is not a true buoyant plume in a classical sense (i.e. a 

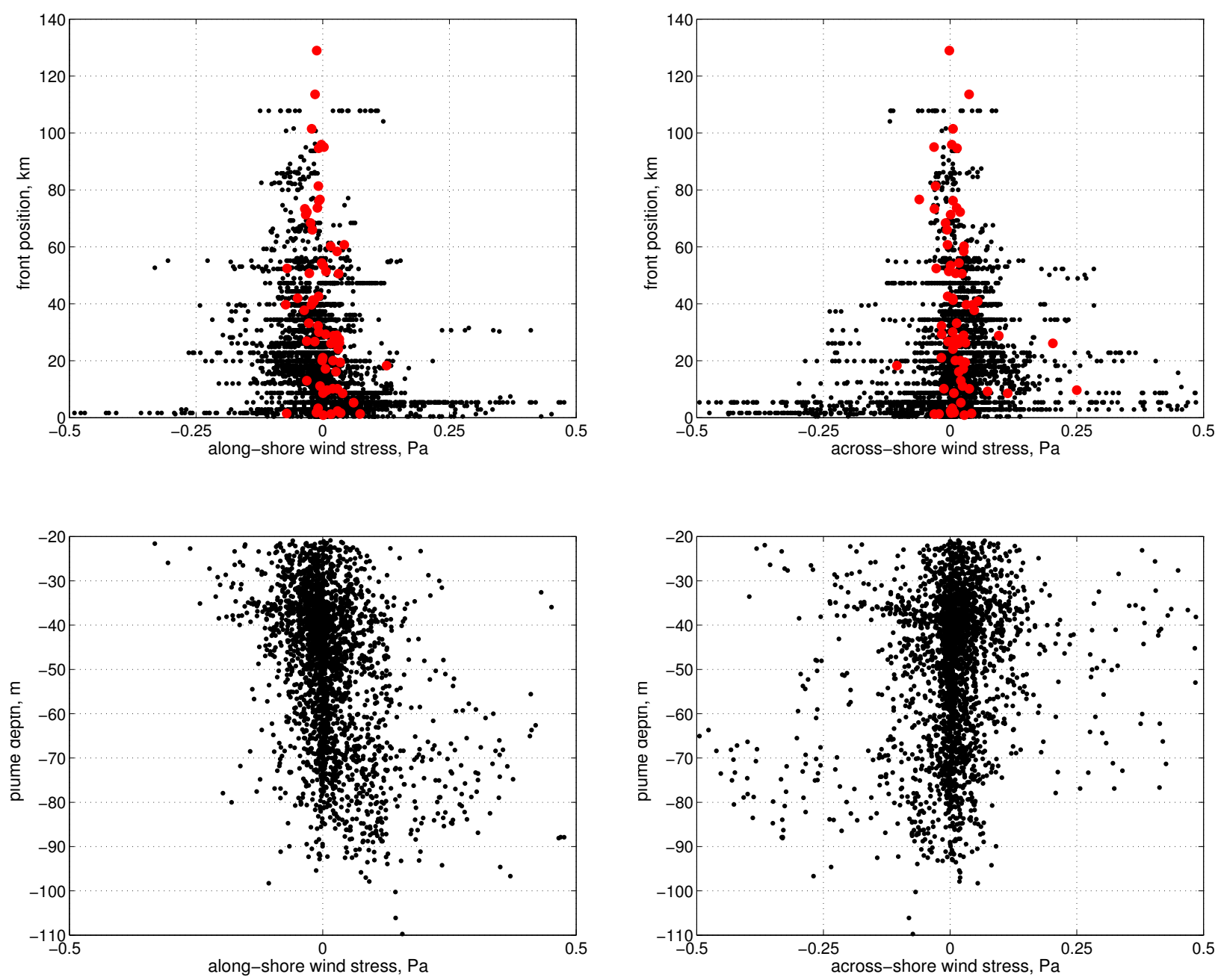

(c) Alongshore windstress, positive is southward (down-(d) Across-shore windstress, positive is offshore, negawelling), negative is northward (upwelling) tive is onshore

Figure 2-20: Plume width (km, upper) and depth (m, lower) vs alongshore (left) and across-shore (right) wind stress $(\mathrm{Pa})$ for FVCOM in April-June. Correlation coefficients are (in clockwise order starting from the top left) $-.22, .12,-.38, .16$. All p-values are $<0.005$.

pulse of freshwater dumped on the continental shelf by a river which propagates in the direction of coastally-trapped waves, like that discussed in Lentz (2004) and Moffat and Lentz (2011)). The following will proceed assuming that the coastal freshwater is indeed a classical plume, then this assumption will be revisited in the discussion section. 
Observations of the plume off of Cape Cod have shown that it is mostly isolated from contact with the bottom (see figure 2-2), and it will be assumed that the plume is "surface trapped". The system is two-layered, in which the active plume overlays a quiescent interior ocean. The theory focuses on the region behind the nose of the plume, which is in geostrophic and hydrostatic balance so that the alongshore Coriolis force $f u$ is balanced by an across-shore pressure gradient. From here on, $\rho_{i}$ is the density of the plume (assumed uniform within the plume), and $\rho_{0}$ is the density of the interior (which is assumed motionless).

\section{Review of two-dimensional theory: The "no entrainment", "some entrain- ment" and "full entrainment" models}

In previous work (Lentz (2004), Moffat and Lentz (2011)), an assumption of alongshore uniformity is used so that $\frac{\partial}{\partial x}=0$, which will be retained here. Other parameters include the bottom slope $\alpha$, the total width of the plume $W_{p}$, the depth of the foot of the front $h_{p}$, the distance from shore to the foot of the front $W_{\alpha}$, and the distance from the foot to the edge of the plume $W_{w}$ (see figure 2-21).

At the onset of wind (or when the wind stress magnitude increases), a mixed layer forms (or deepens) at the edge of the plume of a depth $\delta_{e}$ given by (following Pollard et al. (1972)):

$$
\delta_{e}=\left(\frac{\tau^{2} z_{i}}{\rho_{0} f^{2} g \Delta \rho}\right)^{1 / 4}
$$

where $\tau$ is the wind stress, $z_{i}$ is the initial depth of the plume, $\rho_{0}$ is the reference density, $f$ is the Coriolis parameter, $g$ is the gravitational constant, and $\Delta \rho$ is the density anomaly of the plume.

In the "no entrainment" version, the depth of this layer and the Ekman transport determine the rate at which the edge of the plume moves (assuming the Ekman transport is distributed over the depth $\delta_{e}$ ). It is important to note that equation 2.1 assumes some entrainment when the mixed layer forms. In the "no entrainment" version of the theory, we ignore the density change due to this entrainment and only 

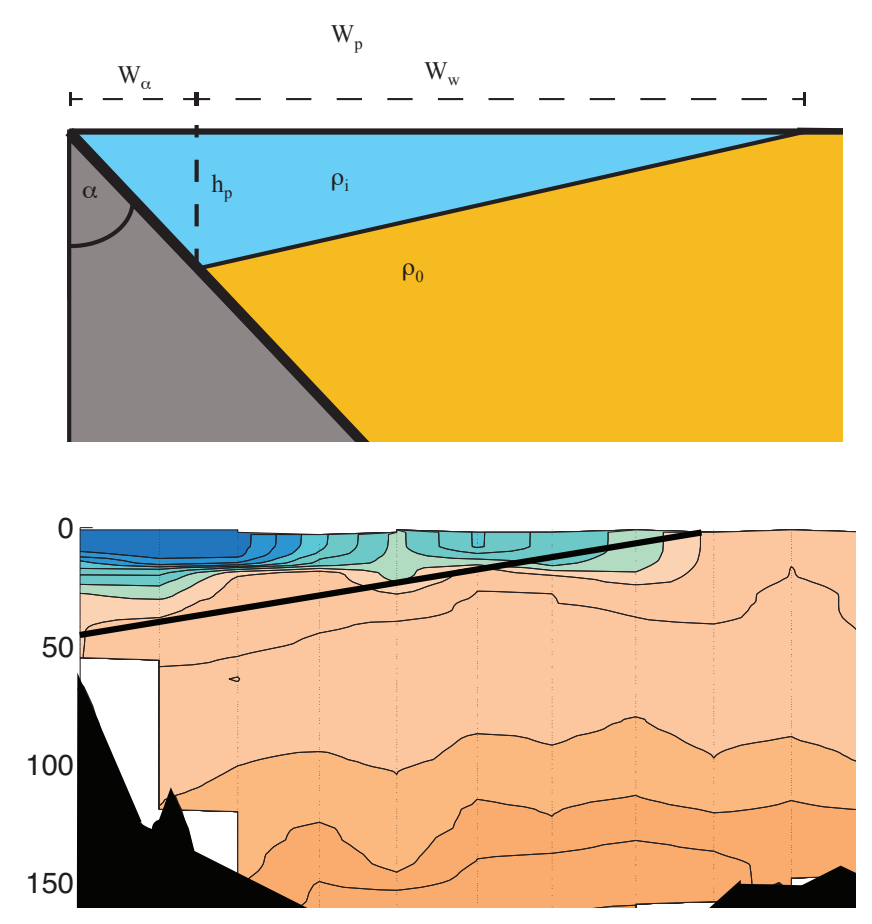

Figure 2-21: Top: Plume parameters, as defined in the text. Bottom: Section of salinity off Cape Cod (as in figure 2-2 showing surface freshwater, which is approximated by the idealized plume on the left.

use this scale to determine the movement of the plume (similar to Fong and Geyer $(2002))$.

In the versions of the theory including entrainment, the formation of this mixed layer leads to entrainment of ambient interior water into the plume. The crosssectional area after this entrainment is simply the initial plume area plus the area entrained by the newly formed mixed layer $A_{e}$ :

$$
A_{o}=\left\{\begin{array}{cc}
A_{i}+A_{e}=\frac{1}{2}\left(W_{p} h_{p}+W_{e} \delta_{e}\right)=\frac{1}{2}\left(W_{p} h_{p}+\frac{\delta_{e}^{2}}{\gamma}\right) & \delta_{e}<h_{p} \\
A_{i}+A_{e}=\frac{1}{2}\left(W_{p}\left(h_{p}+h_{f}\right)-\frac{\left(h_{f}-\delta_{e}\right)^{2}}{\alpha}\right) & h_{p}<\delta_{e}
\end{array}\right\}
$$

where $\gamma$ is the isopycnal slope and $h_{f}$ is the bottom depth at the edge of the plume:

$$
h_{f}=\alpha W_{p}=\alpha\left(W_{\alpha}+W_{w}\right)=h_{p}+\alpha W_{w}=h_{p}\left(1+\frac{\alpha}{\gamma}\right)
$$


The entrainment of interior fluid into the plume increases the area and reduces the

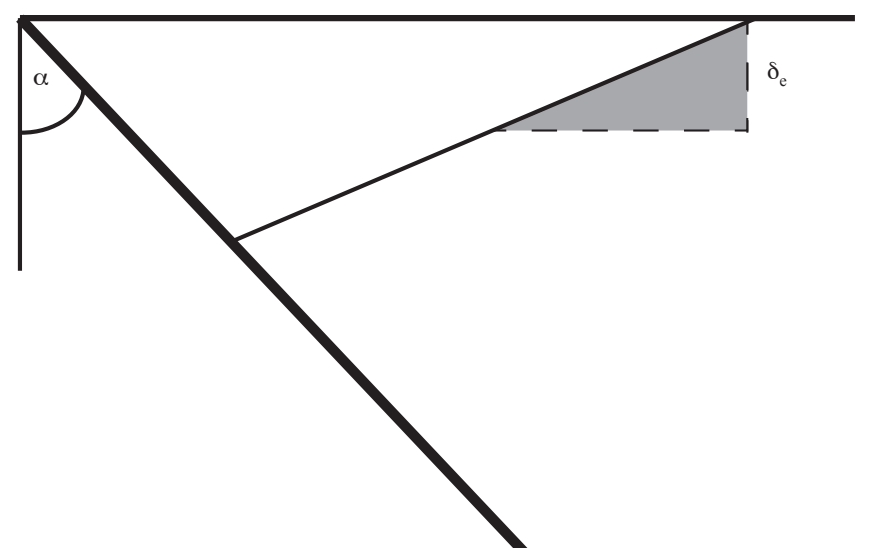

(a) $\delta_{e}<h_{p}$

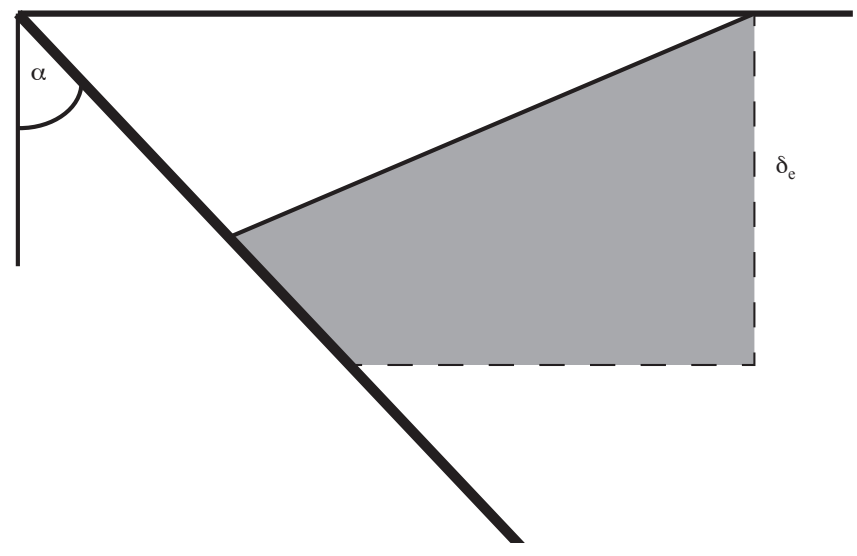

(b) $h_{p}<\delta_{e}$

Figure 2-22: Plume area increases after the onset of winds due to entrainment in the mixed layer. The cross-sectional area of fluid entrained depends upon whether $\delta_{e}<h_{p}$ or $\delta_{e}>h_{p}$

density anomaly of the plume.

Following Lentz (2004), in the "continuous entrainment" theory, an upwelling wind will cause a small amount of entrainment to occur continuously at the offshore edge of the plume after deepening of the wind-mixed layer. When the wind is upwelling-favorable, lighter fluid is transported offshore, which tends to flatten the plume interface. At the offshore edge of the plume, there will be a narrow region where the plume interface slopes upward toward the surface (figure 2-23). Assuming 
that this region is geostrophically adjusted, the width of the region containing the sloping interface is given by the deformation radius:

$$
W_{e}=\frac{\sqrt{g^{\prime} \delta_{e}}}{f}
$$

In this region, the plume interface is shallower than $\delta_{e}$, and so will be mixed with ambient fluid. This would tend to steepen the interface towards the vertical, which counters the effect of offshore Ekman transport of lighter water tending to flatten the interface. This balance can be used to estimate the entrainment into the plume as a function of time. Assuming that the plume slope adjusts on a time scale consistent with geostrophic adjustment $(1 / f)$, the rate at which the area of the plume increases due to entrainment can be derived as in Lentz (2004):

$$
\frac{\partial A}{\partial t}=A_{e} f=\frac{\sqrt{g^{\prime} h} h}{2 f} f=\frac{1}{2}\left(\frac{g \Delta \rho \delta_{e}^{3}}{\rho_{0}}\right)^{1 / 2}
$$

Assuming that the mixing happens stably (in a bulk Richardson number sense, consistent with 2.1), substituting for $\delta_{e}$ gives

$$
\frac{\partial A}{\partial t}=\frac{1}{2} \frac{\tau^{s}}{\rho_{0} f}
$$

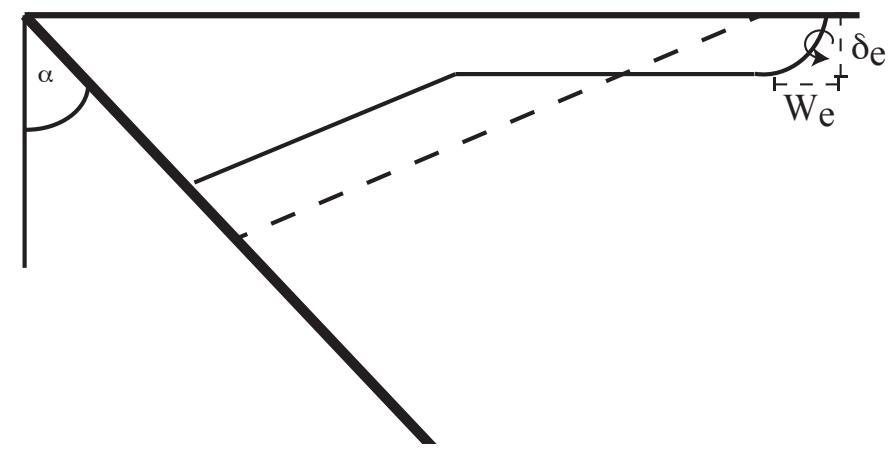

Figure 2-23: Mixing at the edge of the plume during upwellling. Here, competition between the offshore Ekman transport and mixing in the surface layer entrains interior fluid into the plume. 
Moffat and Lentz (2011) have not included continuous entrainment in their theory for the response to downwelling wind; here, a possible mechanism through which continuous entrainment may occur is derived. In contrast to the upwelling case, the Ekman transport and wind-mixing are both working to maintain a vertical plume interface at the edge of the plume. As the plume edge moves towards the coast, however, the depth of the vertical part of the plume interface grows, and the plume gets deeper. From equation 2.1, as the plume depth increases, $z_{i}$ grows, then so too does $\delta_{e}$. As $\delta_{e}$ increases, a small amount of ambient fluid will become entrained into the plume here (figure 2-24). Assuming that the plume interface in the interior between the bottom layer and the surface layer maintains a constant isopycnal slope $\gamma$, a geometric argument gives the cross-sectional area of the fluid entrained:

$$
A=\frac{1}{2} \gamma\left(\frac{\tau t}{\rho_{0} f \delta_{e}}\right)^{2}
$$

Other mechanisms of entrainment during downwelling winds are possible (including

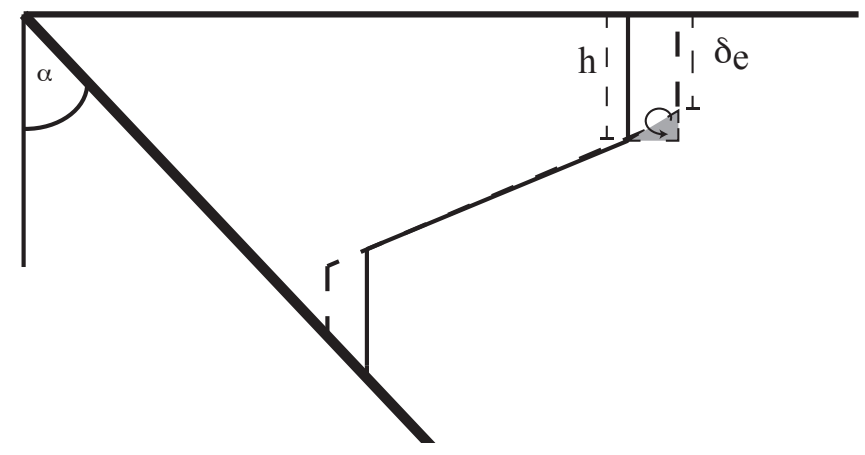

Figure 2-24: Mixing at the edge of the plume during downwelling. Here, deepening of the mixed layer as surface Ekman transport moves the plume edge towards shore leads to entrainment into the plume.

shear-driven mixing or mixing due to instability of the along-shore current, (C. Moffat personal communication)), however these are ignored for now.

In all versions of the theory, the edge of the plume is advected onshore (offshore) for downwelling (upwelling) wind. Integration of the wind stress distributed over the 
surface Ekman layer determines the change in plume width:

$$
\frac{\partial W}{\partial t}=-\frac{\tau}{\rho_{o} f \delta_{e}}
$$

Assuming a triangular cross-sectional plume geometry, plume area and plume width can be used to calculate the depth of the plume.

$$
h_{p}=\frac{2 A_{\text {plume }}}{W_{p}}
$$

Similarly, conservation of buoyancy is used to estimate the density anomaly of the plume for the versions of the theory containing entrainment:

$$
\rho_{f}=\frac{A_{i} \rho_{i}}{A_{\text {plume }}}
$$

where $A_{i}$ and $\rho_{i}$ are the initial plume area and density anomaly.

\section{Accounting for changes in upstream freshwater supply}

In Lentz (2004) and Moffat and Lentz (2011), the cross-sectional area of the plume is conserved except for increases due to entrainment because the supply of freshwater is assumed to be constant. Thus, in the "no entrainment", "some entrainment", and "full entrainment" versions of the theory, the plume area must remain constant or slowly increase, but can never decrease. However, in the coastal ocean east of Cape Cod the supply of river freshwater is of course not constant. Plume area estimates from observations or the model show that the plume area increases and decreases in response to changes in upstream supply of freshwater. These changes may depend upon wind forcing near the source, but also may be completely independent.

The varying supply of freshwater is parameterized in the "upstream" version of the theory using observed ocean salinity at a mooring near Cape Ann (GOMOOS

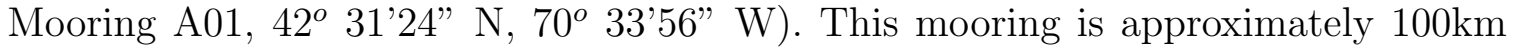
north of the Nauset line, and measures salinity at $11 \mathrm{~m}$ depth. Conceptually, changes 
in freshwater supply will reach the buoy first as they propagate down the coastline. Thus, it may be possible to predict changes in the plume structure at Nauset using information upstream.

Figure 2-25 depicts the average springtime salinity at GOMOOS A01 and the average springtime plume area at the Nauset line. Indeed, it appears that a short time after the salinity at the buoy decreases the area of the plume increases. An empirical

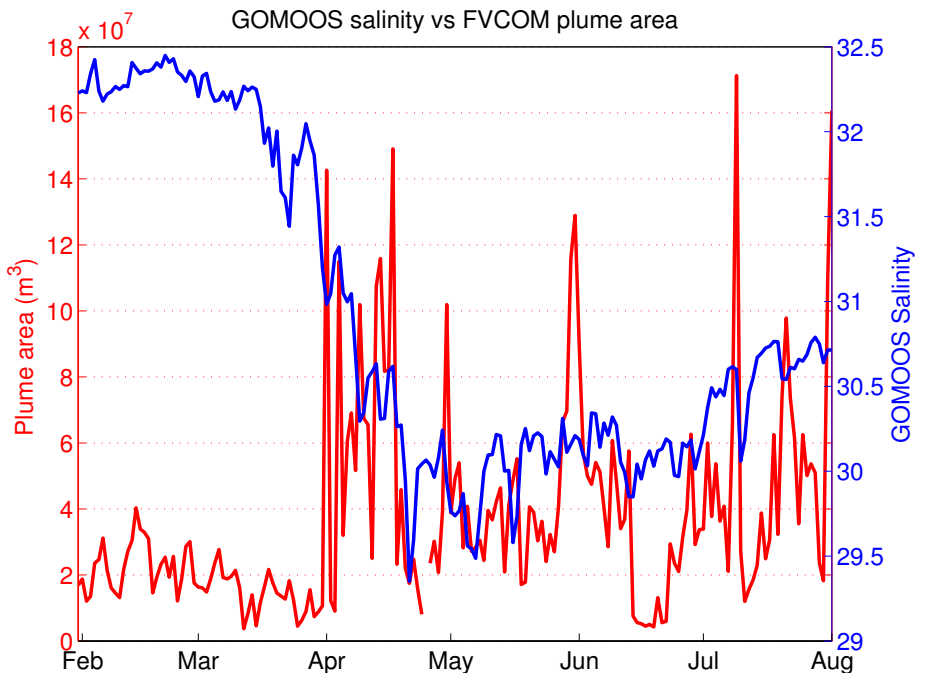

Figure 2-25: Time series of average springtime salinity at GOMOOS A01 (blue) and plume area at the Nauset line (red). Average is taken over February 1-August 1 for the years 2005-2007.

relationship can give an estimate of the changes in plume area at the Nauset line due to changes in upstream conditions as a function of the buoy salinity. It is conceivable that the buoy salinity would not only depend upon the supply of freshwater, but would also vary due to wind. It is assumed that the effect of winds on the observed salinity is of secondary importance to the supply of freshwater, so the buoy salinity is taken to be representative of the freshwater supply.

When estimating the plume area changes due to upstream conditions, a time-lag is applied to the buoy salinity to account for the advective time between the mooring and the Nauset line. Manning et al. (2009) noted a large standard deviation (8 days) in the drifter advective time from Cape Ann to Cape Cod, which indicates that the lag 
time applied should change depending upon changes in the advective time between the mooring and the Nauset line. The appropriate lag time is determined by the lag that gives the maximum correlation to the plume area at the Nauset line for the time period over which the theory is compared to the model (so-called "plume events", described in the next section).

\subsubsection{Wind effect on plume: Application of theory to model}

To apply the theory, "plume events" (times when pulses of freshwater move through the Nauset line) are identified by visual inspection of the surface salinity in the model. There is no strict definition of an "event" here, this simply gives specific time periods for application of the theory. These events last for a few days to more than one week. It appears that these events may be triggered by a combination of enhanced freshwater supply and winds (and thus may be predictable), however this has not been investigated. The lag time between GOMOOS A01 and the Nauset line is determined separately for each event to account for different advective times for different events. The theory is integrated from the beginning of the plume event forward in time for the duration of the event, and changes in plume characteristics are compared to those predicted by the theory.

For example, a plume event occurred following a freshet in early May, 2005 (see figure 2-26). From the end of April till about May 7, the winds were fairly weak, with periods of upwelling-favorable wind. During this time, freshwater from upstream river sources moved down Cape Cod across the Nauset Line. Starting on May 7, the winds shifted to downwelling-favorable and increased in intensity when a large storm system moved through the northeast United States (figure 2-27).

During this period, changes in plume characteristics are compared between the model and the theory. The along-shore wind stress (pictured in figure 2-27) was upwelling-favorable for two days, followed by a period of weak winds which oscillated between downwelling and upwelling winds, and ending with the large downwellingfavorable event. 

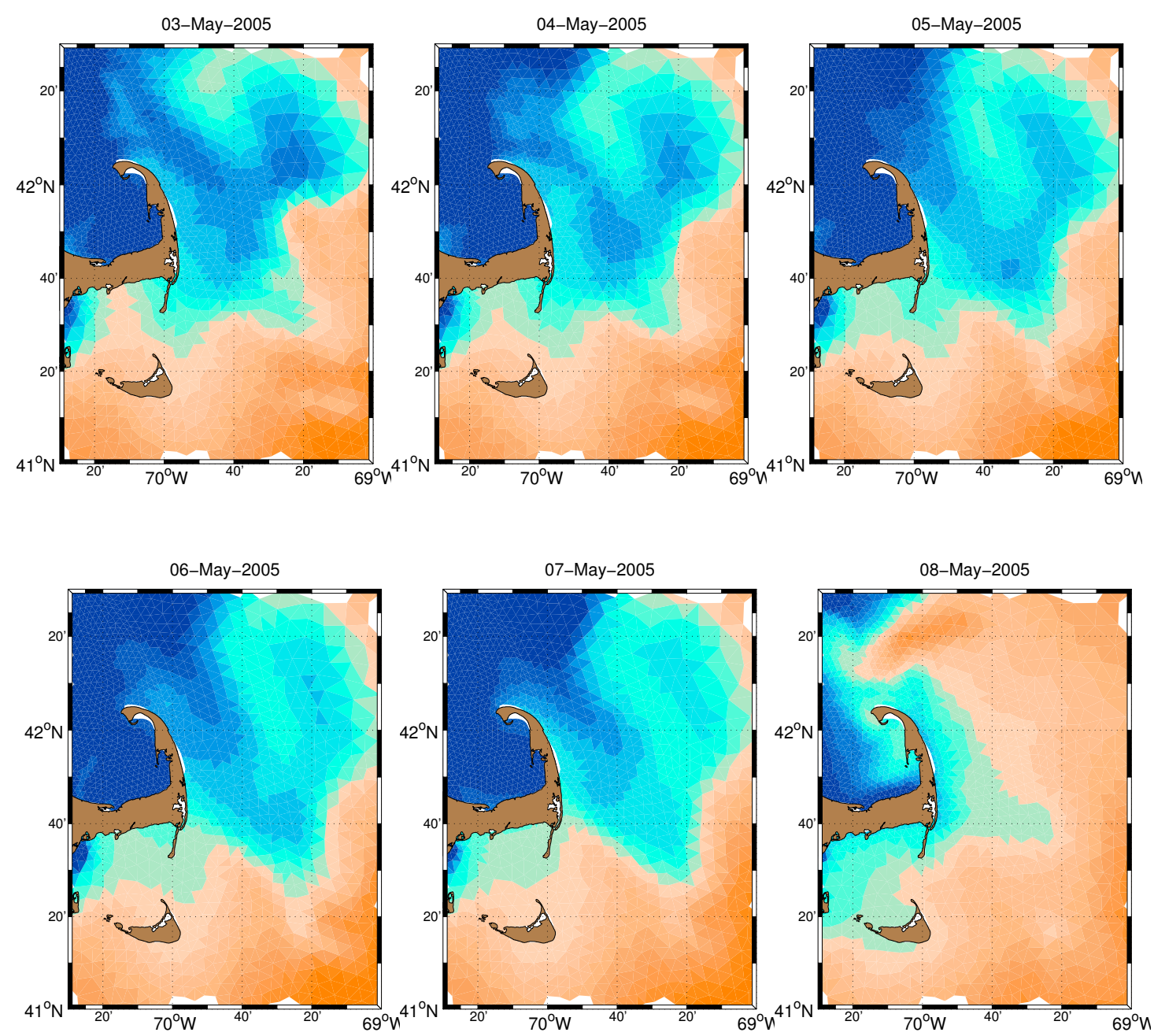

Figure 2-26: Eight snapshots of sea surface salinity from FVCOM from May 2005, showing the plume event described in the text.

Figure 2-28 shows the change in plume width, height, area, and density anomaly as a function of time for the numerical model from May 2 to May 9, 2005. This period corresponds to the wind stress shown in figure 2-27. In all panels, the changes in the model plume are shown in black, while the theoretical predictions are shown in color. The plume width (figure 2-28, panel a) changes in response to the winds in a consistent way to the theoretical prediction described above: upwelling winds make the plume wider, while downwelling winds make the plume narrower. All theories agree here because the plume edge is advected by Ekman velocity, which is independent of the version of the theory. The model plume (black dots) widens for most of the early part 


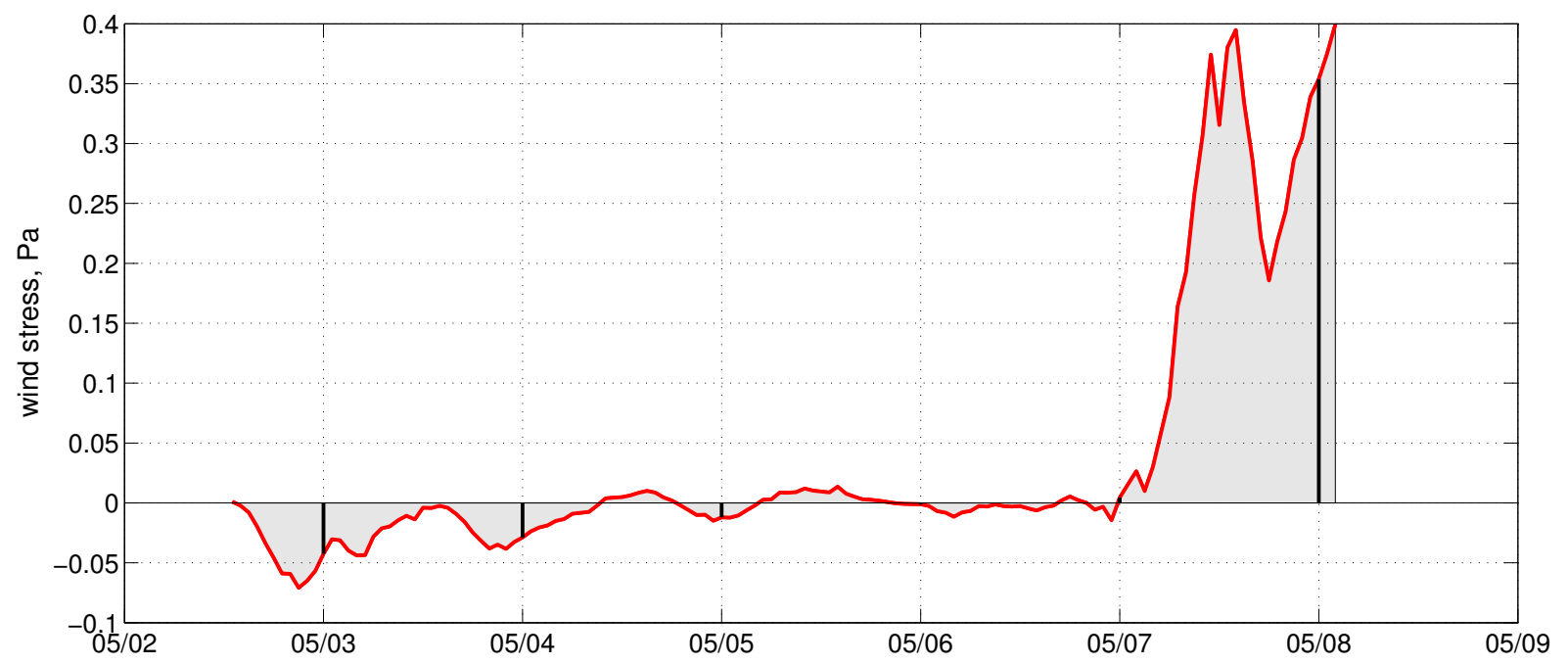

Figure 2-27: Along-shore wind stress for early May, 2005 plume event. Negative (positive) wind stress corresponds to upwelling- (downwelling-) favorable winds. Vertical black lines indicate the times of the 6 snapshots in figure 2-26

of May, but drastically decreases after May 7. The theory mostly predicts plume width changes consistent with the model except for the two short periods of downwellingfavorable wind stress on May 4 and May 5. Also, the narrowing that occurs during the end of the example is under-predicted by the theory.

Changes in the plume area (panel b) show significant differences between theories. The two entrainment theories without an upstream parameterization ("full" and "some") tend to agree with one another, indicating that continuous entrainment at the offshore edge of the plume does not play a significant role in determining plume area as parameterized. For the "no entrainment" version, the plume area remains constant, and in the "some" and "full" models, the plume area can only increase due to entrainment. The model plume area increases for most of the record, but is not well predicted by the theory. At the end of the record, the entrainment theories predict an increase in plume area, while the actual plume area is decreasing; clearly changes in the plume area are not well predicted by the theory.

In the theory, plume depth (panel c) is diagnosed from changes in plume area and plume width (equation 2.9). The versions of the theory including entrainment 


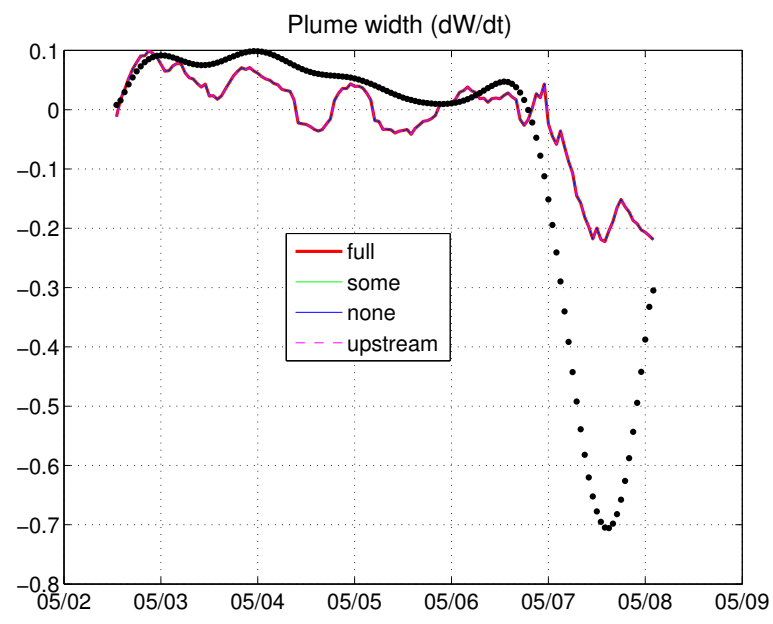

(a) Changes in plume width $(\mathrm{km} / \mathrm{s})$

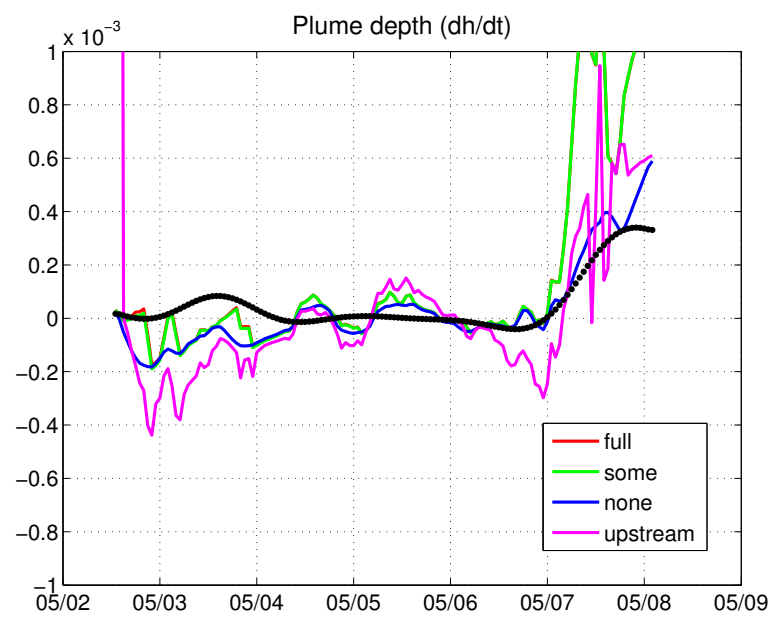

(c) Changes in plume depth $(\mathrm{m} / \mathrm{s})$

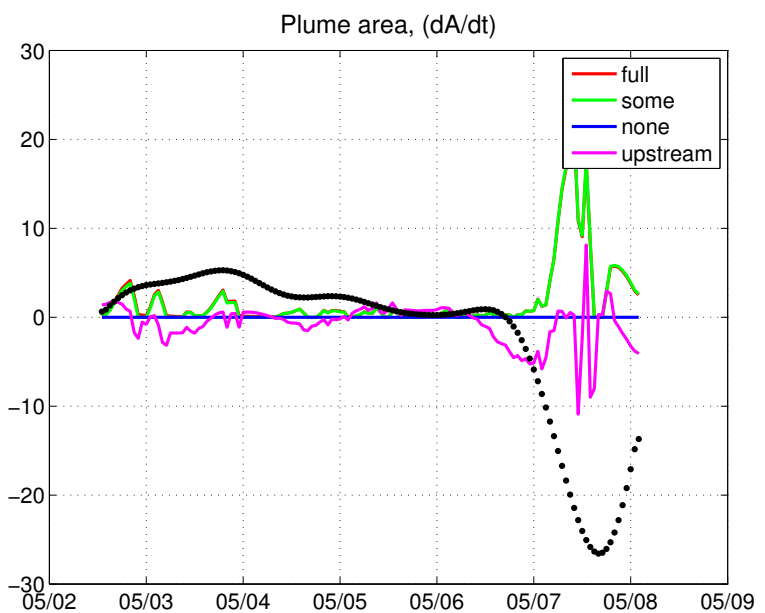

(b) Changes in plume area $\left(\mathrm{m}^{3} / \mathrm{s}\right)$

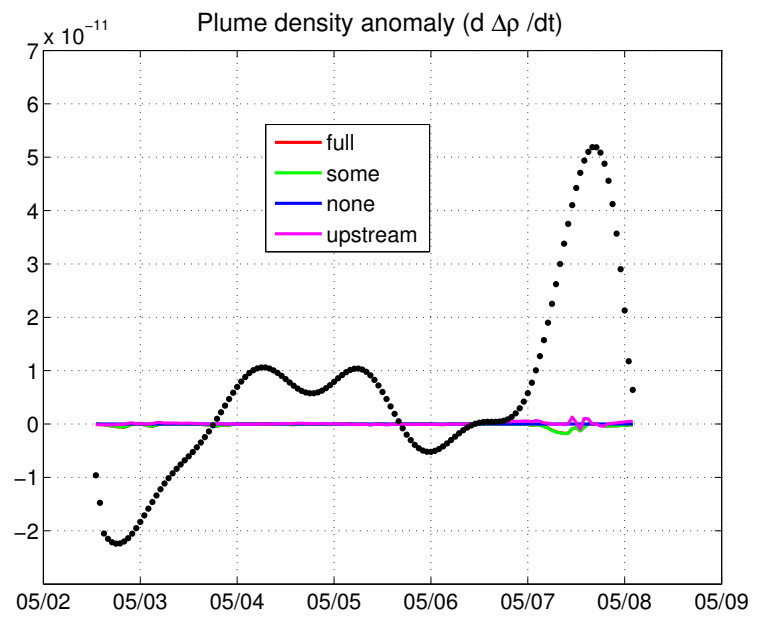

(d) Changes in plume density anomaly $\left(\mathrm{kg} / \mathrm{m}^{3} / \mathrm{s}\right)$

Figure 2-28: Time series of changes in plume characteristics (black dots) plotted versus time with four different theoretical predictions. The "none" (blue) "some" (green), "full" (red) and "upstream" (magenta) theories are shown.

(including the "upstream" version) only predict the plume depth at the end of this example, when the plume depth is increasing as a result of the downwelling wind stress. Earlier in the record, however, there are times during which the plume depth is increasing while the theories predict decreasing.

The theory also struggles to accurately predict changes in the plume density anomaly, which is diagnosed from buoyancy conservation (following equation 2.10). All versions of the theory assume that buoyancy is conserved except through entrain- 


\begin{tabular}{|l|l|l|l|l|}
\hline Characteristic & "None" & "Some" & "Full" & "Upstream" \\
\hline Plume width & $.46,1.34$ & $.47,1.34$ & $.47,1.34$ & $.47,1.34$ \\
\hline Plume area &,-- & $.02(.36), .064$ & $.03(.17), .095$ & $.007(.76), .005$ \\
\hline Plume depth & $.11, .036$ & $.12, .035$ & $.12, .035$ & $.05(.027), .010$ \\
\hline Plume density anomaly &,-- & $-.02(.42),-35.8$ & $.02(.40),-36.95$ & $-.016(.50),-4.6$ \\
\hline
\end{tabular}

Table 2.3: Correlation coefficients and slopes of best-fit lines between each characteristic in figure 2-29 and each of the four versions of the theory (corr coeff, slope). All p-values (except for those shown in parentheses next to correlation coefficients) are $<<.005$. Area and density anomaly are constant for "none" version, thus there is no correlation between the model and this version of the theory.

ment; the fact that the theory performs so poorly indicates that plume buoyancy is not actually conserved in the model, but rather is likely more dependent upon upstream conditions than is even predicted in the "upstream" version of the model.

The same methodology shown in the example plume event in early May, 2005 is applied to 14 different plume events spread out over 3 years of model data. Theoretical predictions of changes in width, depth, area, and density anomaly of the plume are compared to the realistic model, and are plotted against one another for all plume events in figure 2-29. Correlation coefficients and slopes of the best fit line between the theories and model are shown in table 2.3. The patterns in the representative example from June 18, 2004, are largely repeated during all events: the theory best predicts the changes in plume width while exhibiting less skill in predicting the other characteristics. In each figure, the thin black line $(y=x)$ indicates perfect agreement between the model and theory.

\subsection{Discussion and Conclusions}

Freshwater may be found east of Cape Cod throughout the year. This freshwater responds to forcing on time scales ranging from seasonal to daily. A shipboard TSG dataset, a realistic numerical model of the Gulf of Maine, and a simple theory provide useful tools in assessing the variability of the plume on time scales relevant to distribution of important zooplankton species like copepods. 


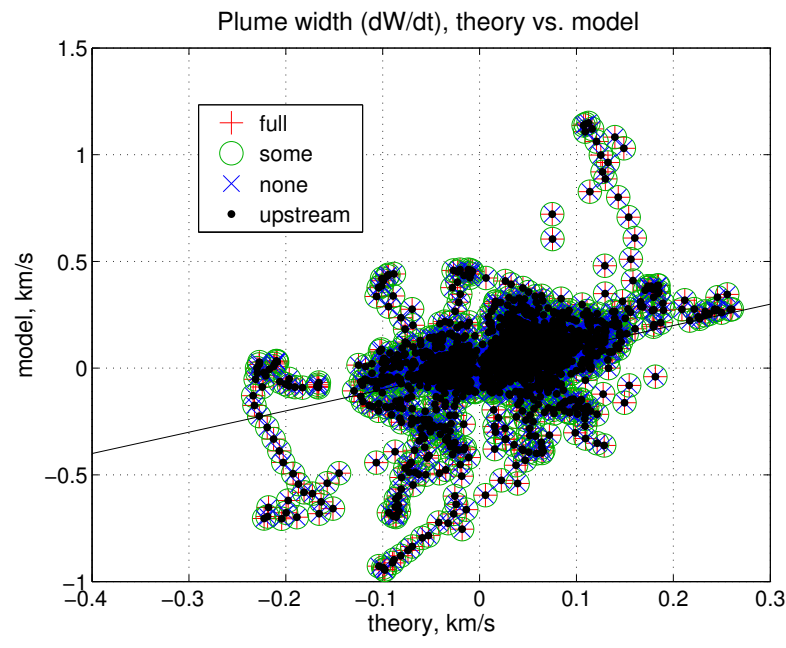

(a) Changes in plume width

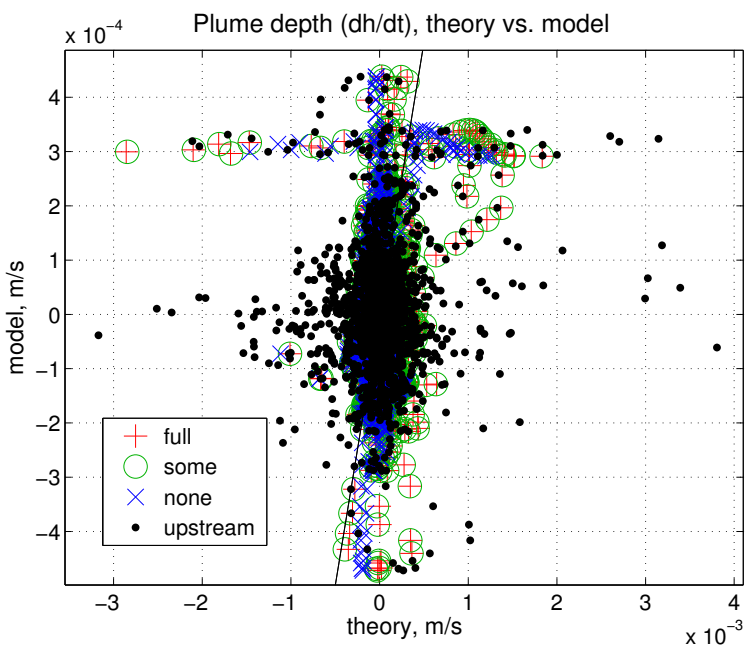

(c) Changes in plume depth

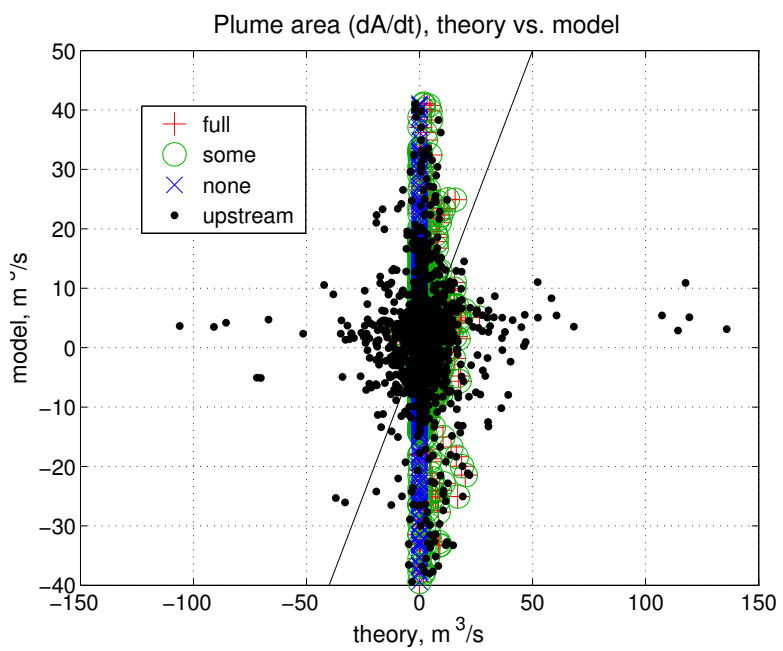

(b) Changes in plume area

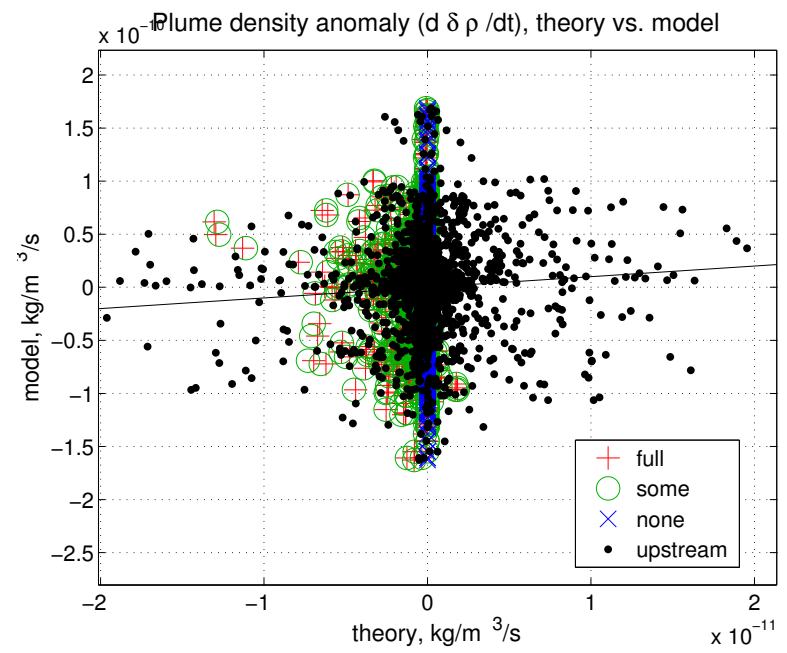

(d) Changes in plume density anomaly

Figure 2-29: Model plume characteristics plotted versus four different theoretical predictions. The "none" (blue x) "some" (green circle), "full" (red +) and "upstream" (black dot) theories are shown. Thin black line indicates 1:1 agreement between model and theory.

The major results of this study are as follows.

- Variability of the surface salinity on seasonal and interannual timescales in the southwestern Gulf of Maine is partly due to river discharge (figures 2-10 and 2-11).

- Freshwater propagates down the coast at the speed of the coastal current, reach- 
ing Cape Cod at a later time roughly consistent with observations of surface velocity (figure 2-12).

- The intensity of the salinity front (magnitude of maximum cross-shore salinity gradient), and the salinity anomaly (difference in salinity inshore and offshore of salinity front) varies seasonally according to the amount of river discharge (figure 2-16).

- The position of the salinity front (distance from shore) varies according to alongshore wind forcing (figure 2-17). Downwelling winds push the front closer to shore, while upwelling winds move the front farther offshore.

- A simple theory for the response of a buoyant plume to along-shore wind forcing is able to predict changes in the position of the edge of the freshwater plume (plume width) for plume events lasting up to 10 days (figure 2-29).

- Including continuous entrainment in the theory (as parameterized in the "full entrainment" version) or a parameterization for the changes in the upstream supply of freshwater do not appear to improve predictability of the plume area (figure 2-29).

- Plume depth and density anomaly are largely independent of wind forcing (figures 2-20 and 2-29).

As mentioned previously, the freshwater east of Cape Cod may not act as a classical buoyant plume. This region is characterized by a background flow that is present even in the winter time (Shcherbina and Gawarkiewicz (2008a)), upon which the riverine freshwater is superimposed. The presence of this flow may be what dictates the depth of the surface freshwater, rather than the wind. Also, some of the strongest tidal flows in the world were found in this region. Vigorous tidal mixing along the bottom may act to fix the depth at which the surface freshwater intersects the bottom ("plume depth" in figure 2-20), thereby making it less susceptible to surface winds. 
Compensating for these factors in the theory may prove to increase the predictive skill.

Another potential problem of the theory is that it assumes that buoyancy is conserved to some extent (recall the assumption of two-dimensionality). We attempted to compensate for that to some extent in the "upstream" model by accounting for a variable river source. This is, however, still only two-dimensional in the sense that three-dimensional processes (e.g. instability of the coastal jet) are completely ignored. These processes can lead to entrainment of interior fluid into the plume, or acceleration of the jet, or any number of other affects that would cause the two-dimensional theory to fail. Additional work is required to understand how the dynamics of a fully three-dimensional plume system were different from a two-dimensional one.

The variability of the coastal freshwater near Cape Cod discussed here may be important for dense copepod aggregation dynamics. The life cycle of copepods in the Gulf of Maine is such that many organisms will be in the surface waters in the springtime (Tarrant et al. (2008)), when the riverine input is large. Copepods in the surface waters near riverine sources may then become embedded in the freshwater plume and be subsequently advected with the freshwater from the north to Cape Cod. Forcing that adjusts the structure of the surface freshwater discussed above may also re-distribute copepods at the surface to make them more readily available for predators. Although the work presented here does not focus on variations in surface freshwater on longer (interannual) time scales, other studies (Balch et al. (2012) and Li et al. (2013)) have indicated that the surface waters in the GOM may be getting fresher due to increased precipitation. It remains to be seen how this change will alter the salinity front structure near Cape Cod, but it nonetheless may be important for the resulting copepod distribution in this region.

The seasonal evolution of the surface freshwater occurs in conjunction with the seasonal growth of the copepod population. It was noted above that continuous entrainment at the edge of the plume did not appear to be much of a factor affecting the plume shape, which may indicate that the coastal freshwater isn't drastically 
mixed with interior water except during stronger wind events. Copepods contained in the surface freshwater may tend to stay contained, which would result in copepods traveling with this freshwater.

In fact, the salinity front itself may provide a mechanism by which copepods aggregate. Epstein and Beardsley (2001) showed that a convergent front, like that found east of Cape Cod, might cause an aggregation of plankton to form via an interaction with downwelling flow and upward swimming behavior. If this process is occurring at the salinity front, then the location of the front, as determined by wind forcing and upstream conditions, may contain aggregations of plankton upon which whales and fish may feed. Also, the strength of the front (as determined by the cross-shore gradient in salinity) may play a role in determining whether or not aggregations form/are maintained there, and how long this process may take/last.

This work provides a framework through which the variability and evolution of the freshwater plume may be understood. Recall, however, that we care about the variability of this front because of its possible role in aggregating copepods. In the following chapter we will investigate the possibility of copepod aggregations forming at or near salinity fronts in the GOM. 


\section{Chapter 3}

\section{Idealized study of copepod aggregation at the edge of a buoyant plume}

\subsection{Introduction}

Right whales, fish, and bird species feed upon copepod aggregations in springtime in the Great South Channel (GSC) in southwestern Gulf of Maine. These aggregations form an important part of the marine ecosystem, yet little is known about the mechanisms-be they physical, biological, or a combination-that lead to these dense aggregations.

The role of convergent fronts in aggregating zooplankton has been the focus of several different studies (Olson and Backus (1985), Franks (1992), Epstein and Beardsley (2001),Genin et al. (2005)). While the details of each investigation differ, the main message is consistent between them: as neutrally buoyant or actively swimming organisms are swept towards a convergent front, the concentration of organisms will increase at the front as the flow sinks and the organisms are left behind (either by actively swimming or being constrained to a depth surface). Schematically, this is shown in figure 3-1: 


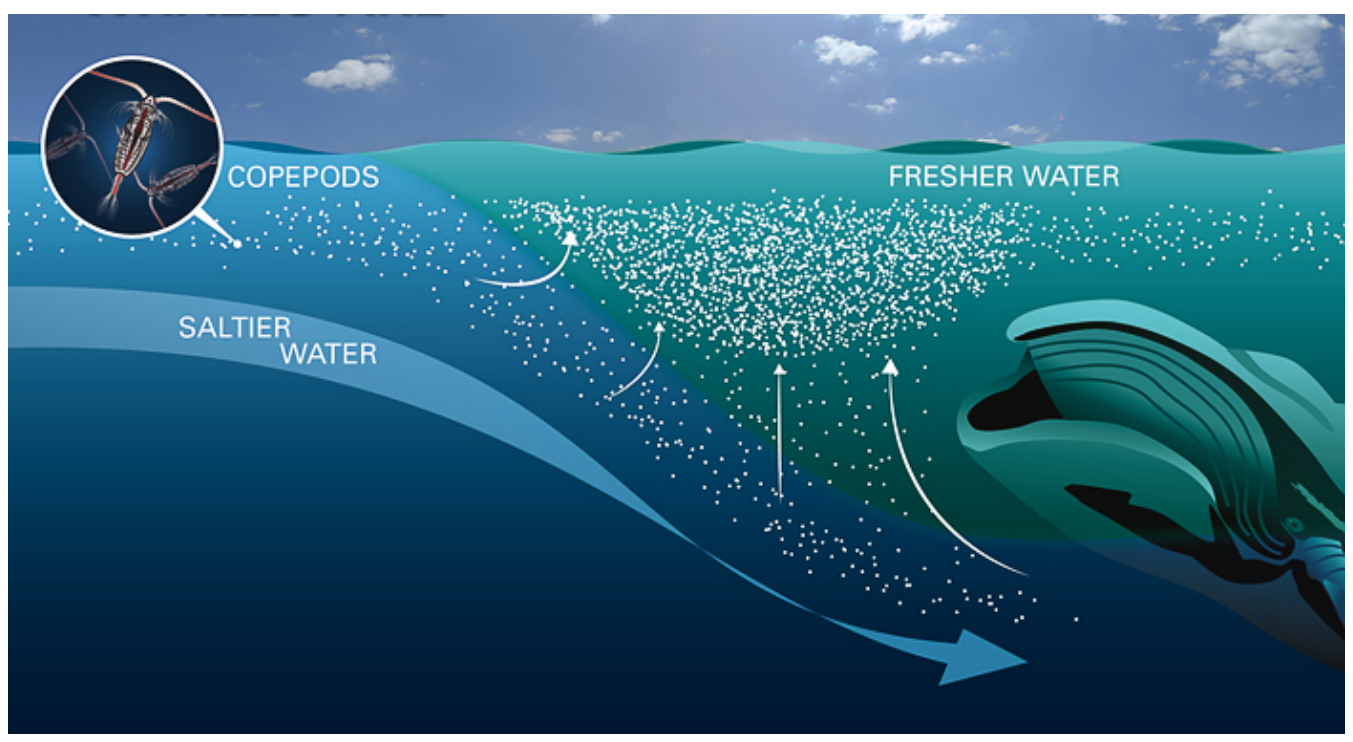

Figure 3-1: Copepods (white dots) near the surface in the vicinity the density front between a buoyant plume and the saltier interior. As the plume advances, the heavier interior fluid sinks beneath the plume. Copepods, wishing to remain near the surface, swim upward against this downward flow. As more copepods are advected into the frontal region, a dense aggregation forms (Illustration by Amy Caracappa-Qubeck, WHOI)

Epstein and Beardsley (2001) applied such a mechanism specifically to the problem of copepod aggregations in the GSC. Using a simple model of a convergent front, they were able to show that copepods swimming to a target depth would increase in concentration at the location of the front. The front in their model is, however, perhaps not realistic to those found in the southwestern Gulf of Maine. They prescribed the frontal circulation in their model, but it is not clear how well this circulation compares to that of real plumes. They assumed along-front uniformity, which is certainly not the case for river plumes, which are limited in both their alongshore and across-shore extent. How does this front-relative circulation change the conceptual model?

Salinity fronts are often found in the Gulf of Maine, especially in the spring time. River runoff forms buoyant plumes which can propagate many kilometers down the coast, stretching even into the GSC (see chapter 2). Is a situation such as that described by Epstein and Beardsley (2001) actually occurring at the edge of a river 
plume? In this chapter, I will investigate under what physical and biological conditions a dense copepod patch may form at the edge of a buoyant plume. Specifically, I will address the following questions:

1. What parameters of an idealized river plume/depth-seeking copepod system (e.g. incident freshwater flux, density anomaly within plume, stratification in the interior, target depth of the copepods etc.) control whether the copepod concentration increases at the plume front?

2. How is the movement of a copepod maintaining a constant depth different from a copepod that is constrained to a constant density surface, or a copepod drifting passively with the flow?

In the following I will use a combination of simple physical models of buoyant plumes coupled to simple copepod concentration and individual copepod models. The work herein follows along that of Epstein and Beardsley (2001), yet is different in a few key ways: The first question focuses specifically on river plume fronts, as opposed to the generic convergent fronts seen in other studies. The river plumes and circulation in this study evolve from a river source to closely simulate the actual front found in nature, whereas in Epstein and Beardsley (2001), the front is twodimensional (across-front distance and depth) and the circulation is kinematic, not dynamic (i.e., it is prescribed beforehand, rather than allowed to evolve). The second question focuses on subtle but perhaps important differences in how particles and copepods behave in the environment. In Epstein and Beardsley (2001), the copepods were assigned a depth-keeping behavior, whereas in this study we compare depthkeeping behavior with completely passive and isopynal-following "behaviors". There is some evidence (see below) that copepods may sometimes reside on surfaces of constant density, as opposed to swimming actively to a certain depth, which may result in different distributions of copepods.

The structure of this chapter is as follows: section 3.2 motivates the subsequent sections by showing realistic plumes in the southwestern GOM and some anecdotal 
evidence for copepod patchiness there. Section 3.3 outlines the models used in this study, section 3.4 shows the results from the study, where we investigate how copepod distributions depend upon the structure of a more complicated front/plume structure and different copepod "behaviors". In section 3.5, we re-evaluate the two-dimensional fronts similar to that of Epstein and Beardsley (2001), and attempt to reconcile the differences between two- and three-dimensional systems; this is followed by discussion and a conclusion in section 3.6.

\subsection{Plumes and convergence in the southwestern GOM}

Epstein and Beardsley (2001) showed some anecdotal evidence for aggregation at a salinity front in observations collected in the southwestern GOM, and suggested that the circulation associated with the salinity front may be responsible. In the introduction it was suggested that a propagating buoyant plume could generate an aggregation, however there are many prerequisites for this to be the case. First of all, the copepods must be behaving in a certain way, namely swimming to stay near the surface. Can we see this behavior in the observations? Also, most of the whale sightings (and, by proxy, the copepod aggregations) are not found near the mouth of rivers, but rather some distance downstream. Do the river plumes in the southwestern Gulf of Maine propagate far enough away from their sources to reach the location in the Great South Channel where right whales are most often seen (see figure 1-2)? And does the horizontal convergence at the edge of the propagating plume remain large enough for an aggregation to form? Before we dive into the models, we first explore these questions to determine the feasibility of the proposed mechanism. This section details some simple analysis of a realistic numerical model and autonomous underwater glider observations that show that the necessary pieces for the proposed mechanism are in place in the southwestern GOM 


\subsubsection{Observations of a freshwater plume and copepod be- havior in the GSC-autonomous underwater gliders}
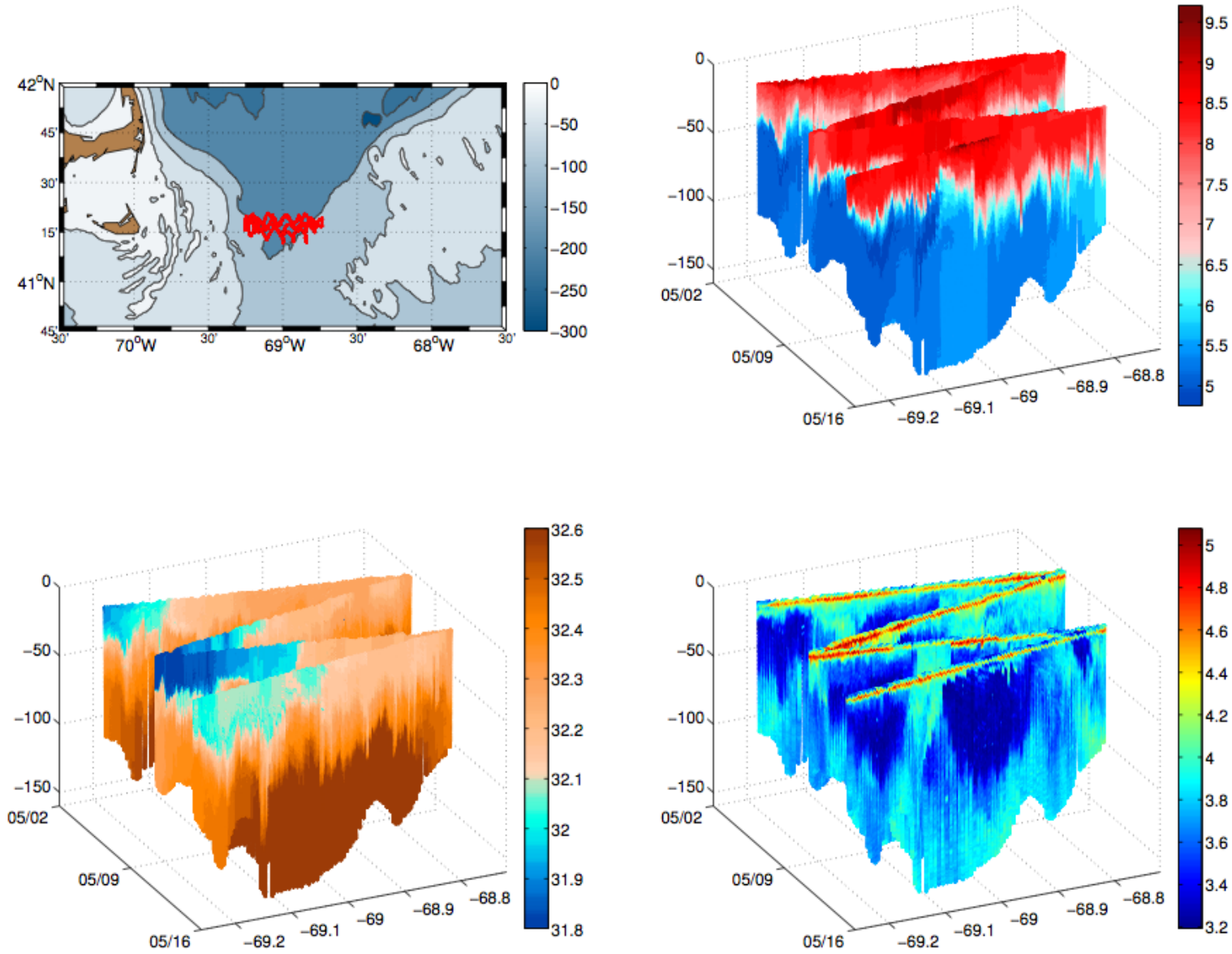

Figure 3-2: Time-depth-longitude plots of temperature (upper right), salinity (lower left) and acoustic backscatter (lower right) from glider we04 in May, 2010

In 2010, three gliders were deployed in the GSC. One glider, we04, performed zonal across-channel sections, each time crossing from the nearshore waters nearly to Georges Bank. Figure 3-2 shows the path taken by the glider, as well as time-depth sections of temperature, salinity and acoustic backscatter. First, one may notice that a thermocline at about $20-50 \mathrm{~m}$ depth is present throughout the glider's deployment; this is a result of springtime warming of the surface waters. The second thing to notice is that the surface waters are freshest on the western half of the glider line, 
especially during the middle portion of the deployment. This freshening is the result of a freshwater plume propagating down the coast of Cape Cod into the GSC, one of the criteria necessary for the proposed mechanism to occur.
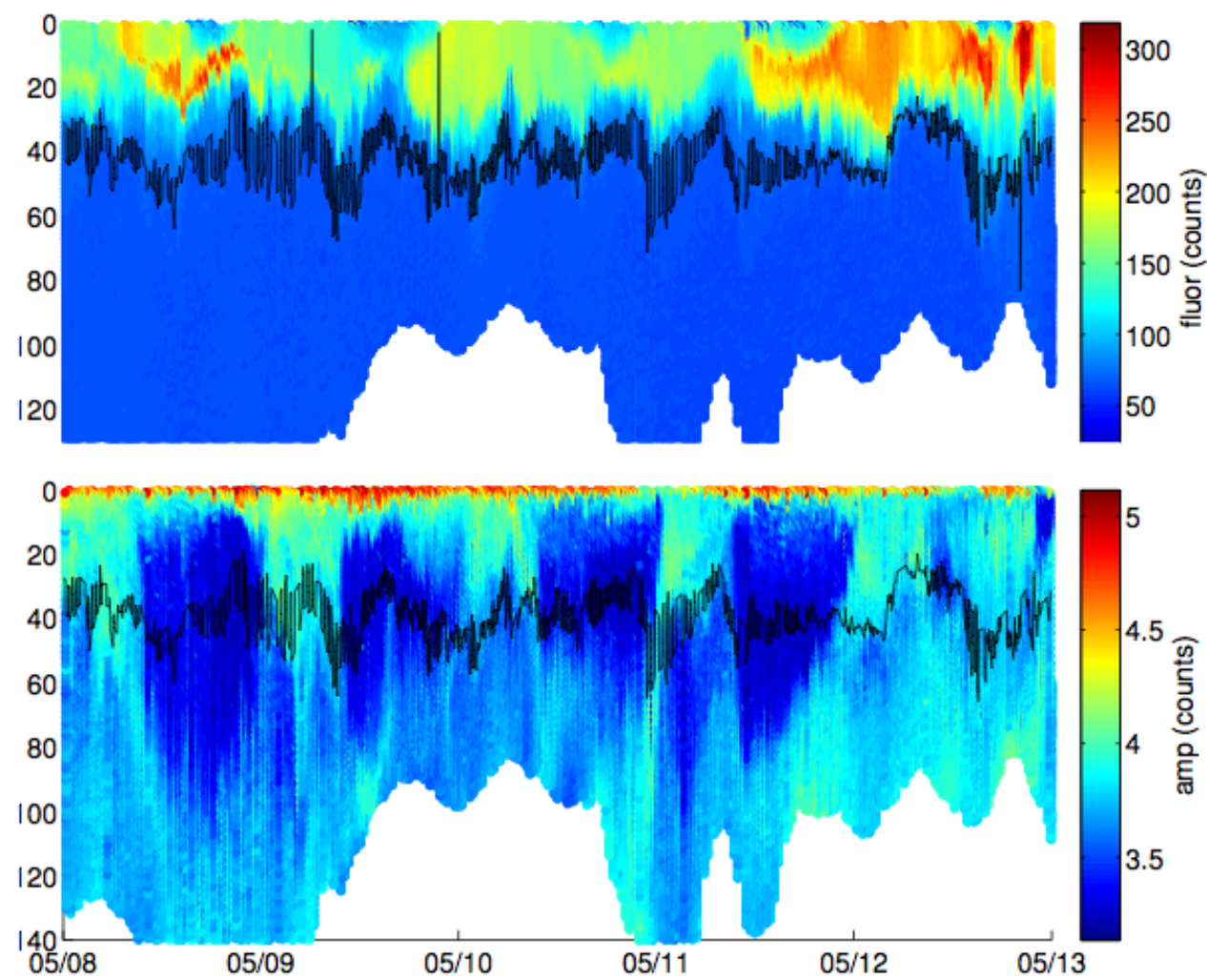

Figure 3-3: Chlorophyll fluorescence (top) and acoustic backscatter (bottom) from glider we04 in May, 2010. Black line is the $\sigma=25.4 \frac{\mathrm{kg}}{\mathrm{m}^{3}}$ contour

In addition to the physical parameters, we are able to observe optical and acoustic proxies of biological fields from the glider. Pictured in figure 3-3 is the chlorophyll fluorescence and $1 \mathrm{MHz}$ acoustic backscatter from the glider. The fluorescence is highest in the surface waters, reflecting the fact that most of the phytoplankton are found there. The thickness of the layer of phytoplankton sitting at the surface increases and decreases roughly as the $25.3 \mathrm{~kg} / \mathrm{m}^{3}$ density contour (shown in black) moves up and down, indicating that the phytoplankton are moving up and down in the water as the density surfaces rise and fall. 
The acoustic backscatter from the glider's ADCP also shows some interesting patterns. The strength of the backscatter at the ADCP's frequency $(1 \mathrm{MHz})$ gives some information about the number of sound scatterers in the path of the acoustic beam, whether they are bubbles, detritus, or copepods. First, the strong backscatter signal from the surface is readily apparent. It is not possible to distinguish between the surface signal and copepods, some of which may be sitting at or very near the surface. Similarly, the enhanced backscatter near the bottom could be due to suspended sediment or copepods resting in the bottom waters. We can, however, see that there appears to be a group of high scatterers moving up and down in the water column. The periods when this patch is high in the water correspond to night time, and vice-versa. These are likely copepods performing diel vertical migration (DVM). DVM is a well-known behavior whereby copepods swim to the surface at night presumably to feed on phytoplankton, but swim to depth during the day to avoid predators. These observations are consistent with similarly collected data presented in Baumgartner and Fratantoni (2008), and provides evidence that copepods are indeed actively swimming in the southwestern GOM, another of the prerequisites for the aggregation mechanism to occur.

Perhaps most interesting is what happens to the copepods when they are residing near surface at night, after having swum up from depth. During these times, the bottom of the copepod layer appears to also be moving up and down with the 25.3 isopycnal, similar to the phytoplankton. This is consistent with the idea that the copepods are swimming to the surface to feed: If the phytoplankton are "riding" the isopycnals up and down, then it makes sense that the copepods would also.

The vertically migrating copepods observed in 2010 are swimming, but not always swimming to the surface, which is another part of the requirement for the hypothetical mechanism. Is there other evidence for copepods staying near the surface? Baumgartner et al. (2011) showed using a combination of video plankton recorder and optical plankton counter measurements that at several stations throughout the southwestern GOM copepods were found sitting at or just near the surface (within a few meters). 
It is not clear from these images, however, whether the copepods were swimming to stay near the surface, or were simply lying on a density surface. While the active swimming behavior has been the focus of other studies on particle movement near two-dimensional convergent fronts, the movement of particles resting on a constant isopycnal has not received as much attention, and will be addressed in this work.

\subsubsection{Propagation of the plume (and associated convergence) far downstream-FVCOM simulations}

Observations have shown that freshwater plumes do indeed propagate into the southwestern GOM (chapter 2), but what we cannot see is whether there is appreciable horizontal convergence at the edge of the plume. For this, we turn to realistic model simulations (FVCOM, Chen et al. (2006)). Figure 3-4 shows Hovmoller diagrams

of salinity and horizontal convergence $\left(\frac{\partial w}{\partial z}\right.$, detided with a 38-hour low-pass filter) offshore of Nauset, MA, in late spring 2005 from FVCOM. As a plume of freshwater propagates across the Nauset section starting on May 29, the salinity near the coast decreases, and the edge of the plume (the boundary between the blue and tan waters) moves offshore (to about $25 \mathrm{~km}$ from shore). At the same time, we see a prolonged (approximately 1 week) period of enhanced convergence just at the offshore edge of the plume.

Similar relationships between convergence and salinity front events can be seen throughout the spring (see figure 3-5). During the salinity front or plume events, the salinity difference across the section increases as the freshwater propagating down the coast crosses the section. During many of these times, a peak in horizontal convergence can be seen. Although this evidence is only circumstantial, it suggests that freshwater plumes could potentially play a role in creating dense copepod aggregations. Additionally, this shows that buoyant plumes can propagate far from their sources, reaching into the GSC where whales are found feeding upon copepod patches. 

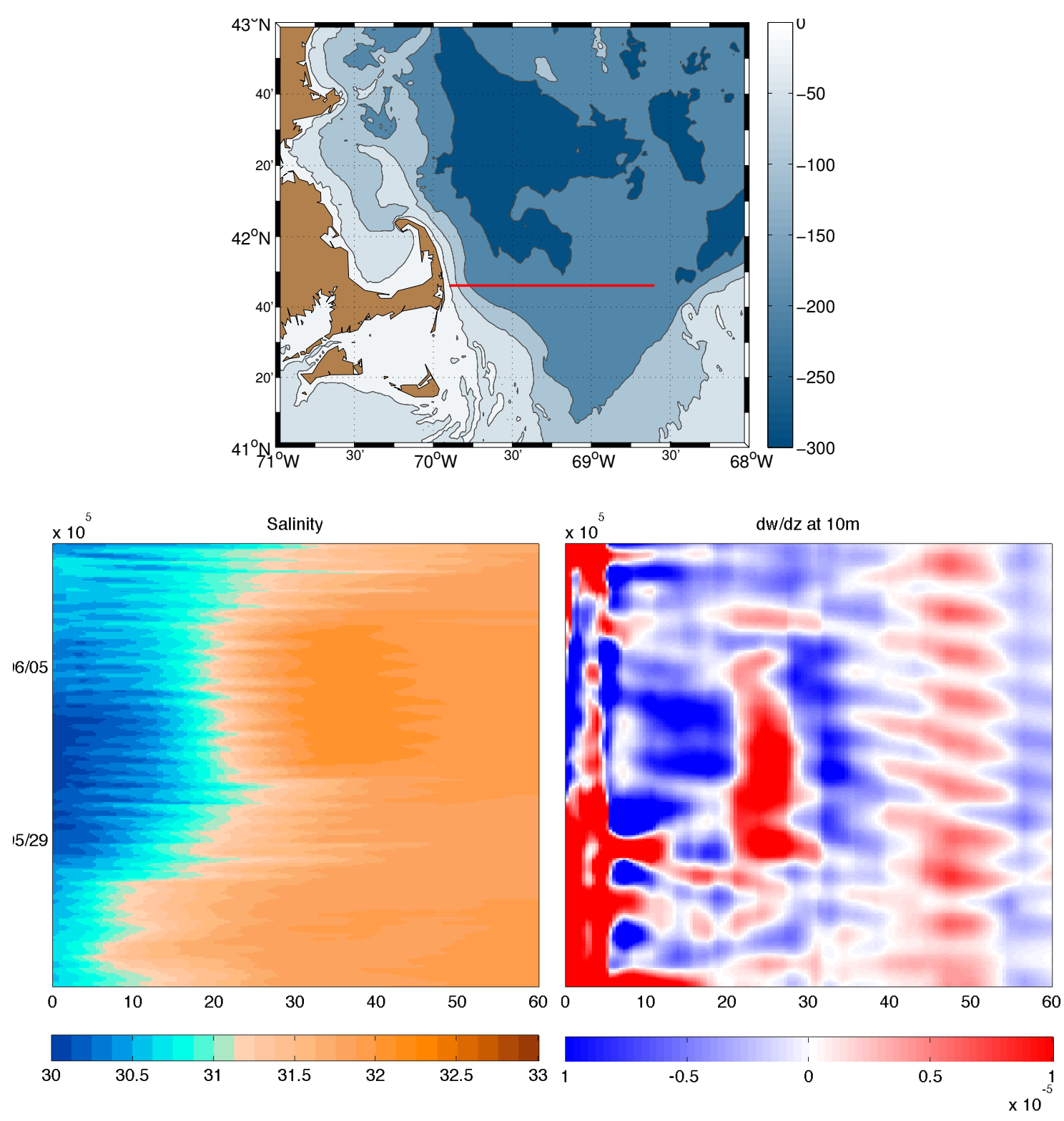

Figure 3-4: Hovmoller diagrams of salinity (left) and detided convergence (right) for the section offshore of Nauset, MA (above). A plume of freshwater crosses the section beginning on May 29. For the week following the start of the event, convergence can be found just offshore of the plume (near $25 \mathrm{~km}$ from shore).

\subsection{Methods}

\subsubsection{5 layer plume and copepod concentration model}

The first model used to study the plume-copepod system is a 2.5 layer model similar to that used in Wood (2008). The model formulation is described therein, and is only 


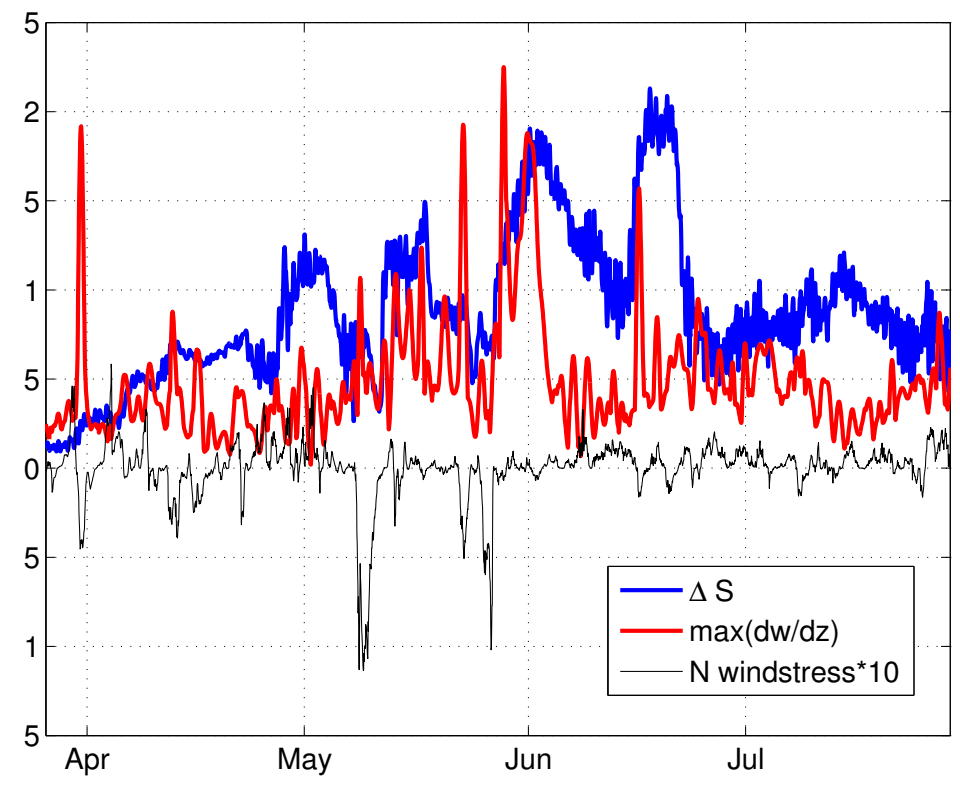

Figure 3-5: Maximum salinity difference across the section (blue) and maximum convergence along the section (red). Plume events are seen as periods when salinity difference across the plume increases (indicating fresher water inshore). During many of these times, the convergence increases.

summarized here. The model is hydrostatic and Boussinesq, and within each layer the velocity is vertically uniform. The model solves the vorticity-Bernoulli form of the momentum equations:

$$
\frac{\partial \mathbf{u}}{\partial t}+(f+\zeta) \mathbf{k} \times \mathbf{u}=-\nabla\left(g^{\prime} h+\frac{1}{2} \mathbf{u}^{2}\right)
$$

where $\mathbf{u}$ is the layer velocity (vertically uniform within each layer), $f$ is the Coriolis parameter, $\zeta$ is the relative vorticity, $g^{\prime}$ is the reduced gravity of the layer, and $h$ is the layer thickness. Transports in the model are computed using a flux-corrected scheme, and simple Euler stepping is used for the integration in time. $f$ is set to a constant $\left(10^{-4} \frac{1}{s}\right)$ for all the model runs. The $25 \mathrm{~km}$ by $400 \mathrm{~km}$ model interior is set up with $500 \mathrm{~m}$ horizontal resolution, and the time step is 150 minutes.

The ambient interior water is divided into two layers: a stagnant bottom layer (infinitely deep), and an initially stagnant upper layer that represents a surface mixed 
layer that forms in the GOM in the late spring/early summer. The uppermost layer consists of the freshwater plume, which is initially very thin (not zero for numerical stability). At the beginning of the model run, freshwater starts discharging from a river mouth and propagates downstream as a buoyant current in the direction of coastal-trapped waves. The river source remains on for the entire model run. As the fluid enters the domain, it not only has mass, but some momentum as well (analogous to river discharge impinging on the coastal ocean). Note that there is initially no flow in the domain; this is not consistent with observations in the GOM which show a coastal current present independent of a river plume (Shcherbina and Gawarkiewicz (2008b)). If a background flow were present, the plume may propagate relative to this flow, and so we ignore it here.

Embedded in the physical model we have a simple copepod concentration model, similar to that used in Epstein and Beardsley (2001). In this model, the copepods are constrained to sit at a prescribed depth $d_{b i o}$, and copepod concentration is determined by

$$
\frac{\partial}{\partial t} C+\nabla \cdot(\mathbf{u} C)=\nabla \cdot(\kappa \nabla C)
$$

where $\mathbf{u}$ is the horizontal velocity at the copepod depth $d_{b i o}$, which depends upon the layer in which the copepods reside. This velocity field depends upon whether $d_{b i o}$ is within the plume, the mixed layer, or the deep layer. Initially, the tracer is distributed uniformly across the domain downstream of the freshwater source. This tracer then passively follows the horizontal flow, but is not allowed to deviate from its target depth.

\subsubsection{ROMS plume model}

While the layer model is well suited to understanding some of the simple dynamics of the plume-copepod system, other aspects require a higher level of sophistication. Most notably, in order to understand how the different vertical positioning of the copepods (depth-seeking vs. isopycnal vs. passive) influences concentration, it is 
necessary to use a model with many vertical layers. For this study, we use the Regional Ocean Modeling System (ROMS, Shchepetkin and McWilliams (2005)), a widely used primitive equation model. The model is implemented in a simple setup to simulate the movement of a buoyant plume along a straight coastline.

In our setup, the base model is set up as a re-entrant channel, with a buoyant fluid source on one of the solid boundaries. The domain is rotating at a constant rate, so a buoyant coastal current forms and propagates along the boundary in the sense of coastally-trapped waves.

The domain for our setup is $25 \mathrm{~km}$ wide by $300 \mathrm{~km}$ long, and water leaving one end of the domain enters again at the other end. The solid walls, separated by $25 \mathrm{~km}$, are far enough apart that the plume does not feel the effects of the opposite wall. The horizontal resolution is $500 \mathrm{~m}$ in the alongshore direction and $250 \mathrm{~m}$ in the across-shore direction. The vertical resolution varies from between about $1 \mathrm{~m}$ near the surface to 2 meters at the bottom of the domain, and there are 26 vertical layers. A summary of the salient model parameters is listed here:

- 4th order Akima advection used in vertical and horizontal

- Large-McWilliams-Doney turbulence closure

- Quadratic bottom friction (bottom is flat and is $50 \mathrm{~m}$ deep)

- Model domain is periodic in the along-shore direction, with solid vertical walls on the other two sides boundaries (width $=25 \mathrm{~km}$, length $=300 \mathrm{~km}$ )

- Along and across-shore resolutions are 500m and 250m, respectively.

- Point source (7500 $\frac{\mathrm{m}^{3}}{\mathrm{~s}}$, equivalent to layered model) on one boundary, density is approximately $1000 \frac{\mathrm{kg}}{\mathrm{m}^{3}}$ (fresh water).

- Time step is 30 minutes, model is run until plume nose wraps around model back to source 
The model is initialized with a horizontally-uniform interior density. While the salinity is initially uniform with depth, the vertical structure of the initial temperature profile, shown in figure 3-6 is chosen to be representative of typical springtime conditions in the Gulf of Maine. The thermocline in this setup starts at a depth of about $20 \mathrm{~m}$. In this way, this model setup is different from typical buoyant plume investigations (e.g. Griffiths (1986)), which assume a uniform interior. Figure 3-6 also shows a snapshot of the surface density distribution for the base ROMS model run. The plume source is located $270 \mathrm{~km}$ from the end of the domain. The velocity output from this model run is then used to advect particles using the Larval TRANSport model.
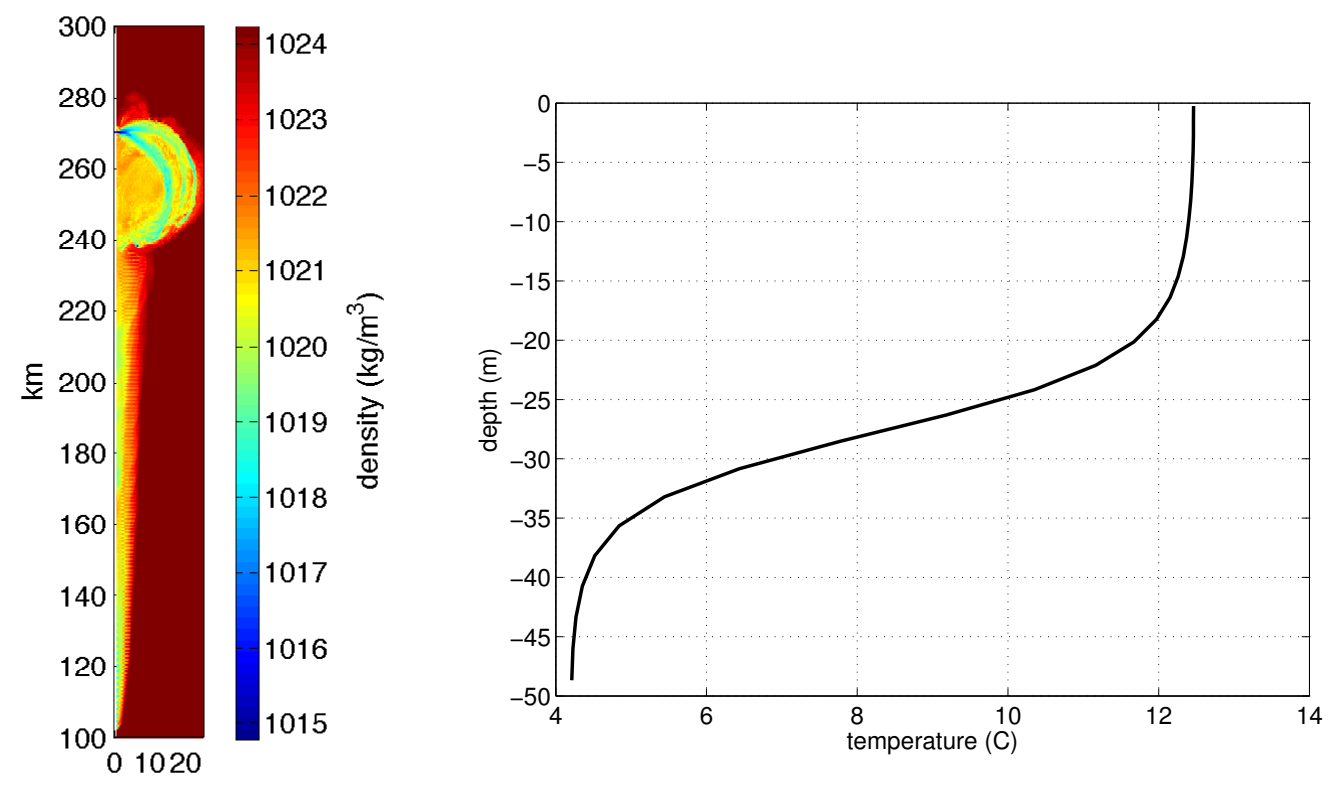

Figure 3-6: (Left) Example snapshot of surface density showing plume propagation down-coast. (Right) Initial vertical profile of temperature.

\subsubsection{LTRANS particle tracking}

Conceptual models of copepod aggregation at a density front (e.g. Epstein and Beardsley (2001)) depend upon the copepods actively swimming to maintain a target depth (analogous to an isobaric or iso-geopotential float). However, as noted in section 3.2, 
observations of copepods indicate that the organisms may not be sitting at a constant depth or geopotential surface, but rather at a constant density surface (analogous to an isopycnal float). Using the base ROMS model run, three different drifter behaviors were implemented using the Larval TRANSport model (LTRANS, Schlag and North (2012)) particle tracking package:

- Depth-seeking-These drifters were constrained to remain at their release depth $(1-35 \mathrm{~m})$

- Isopycnal- These drifters were constrained to remain at their release density

- Passive- These drifters are advected by the full three-dimensional flow

LTRANS uses a water-column interpolation method to determine the water velocity at the exact location of each particle. In this method, water properties (namely $u, v$, and $w)$ are interpolated along the levels of the model to the horizontal locations of the particles, yielding a vertical profile at the particle's location. A tension-spline curve is then fitted to the profile to compute the value at the specific depth of the particle. The model then uses a fourth-order Runge-Kutta scheme in space and time to compute the advection of particles from the model's velocity field. Particles that encounter either a horizontal or vertical boundary in this model are reflected back into the domain.

LTRANS was originally designed to advect particles completely passively (following the three-dimensional flow) or along a depth-surface (following only the horizontal flow at that depth). For this study, we added the isopycnal component. At every time step, the particles follow the three-dimensional flow, as if they were a passive particle, except that at each time step the water density at the particle's location is computed. If the density at the location of the particle doesn't change (or is within $0.01 \mathrm{~kg} / \mathrm{m}^{3}$ of its previous value), then nothing happens. If, however, the density is different by more than $0.01 \mathrm{~kg} / \mathrm{m}^{3}$, then the particle is moved up or down until the density difference is less than this threshold. 
Studies using numerical particle tracking have recently become more popular; other studies have used LTRANS with ROMS output successfully (e.g. North et al. (2006), North et al. (2011)). In this application, three blocks of drifters were released ahead of the nose of the plume, each block assigned a different behavior. The initial dimensions of the block were $250 \mathrm{~m} \times 500 \mathrm{~m} \times 2 \mathrm{~m}$ in the across, along, and depth dimensions, respectively. The plume source is upstream of the block, and the plume propagates through the drifter block, advecting the drifters appropriately.

\subsection{Layer model and ROMS results}

\subsubsection{Layer model validation}

Before continuing with the model, first we test whether the properties of the plume agree with simple theory. Scales for plume depth, width, and nose propagation speed can be derived for a plume incident on an infinitely deep interior with a vertical coastal wall (a 1.5 layer system). As summarized in Lentz and Helfrich (2002), the plume depth at the wall $h_{p}$ scales as:

$$
h_{p}=\sqrt{\frac{2 Q f}{g^{\prime}}}
$$

where $g^{\prime}=g \frac{\left(\rho_{0}-\rho_{p}\right)}{\rho_{0}}, \rho_{p}$ and $\rho_{0}$ are the plume and ambient densities, respectively, $Q$ is the riverine volume flux, and $f$ the Coriolis parameter. The width of the plume scales as:

$$
W_{p}=b_{1} L_{r}=b_{1} \frac{\sqrt{g^{\prime} h_{p}}}{f}
$$

where $L_{r}$ is the deformation radius. Finally, the plume nose advances at a speed that scales like the internal gravity wave speed:

$$
c_{p}=b_{2} c_{w}=b_{2} \sqrt{g^{\prime} h_{p}}
$$


Both $b_{1}$ and $b_{2}$ have been shown experimentally to be $\approx 1$.

To test the layer model, we compare $h_{p}, W_{p}$, and $c_{p}$ from equations $3.1,3.2$, and 3.3 to the parameters of the plume diagnosed from the model run with different initial conditions and plume forcing. It is important to note here that we take the "ambient"

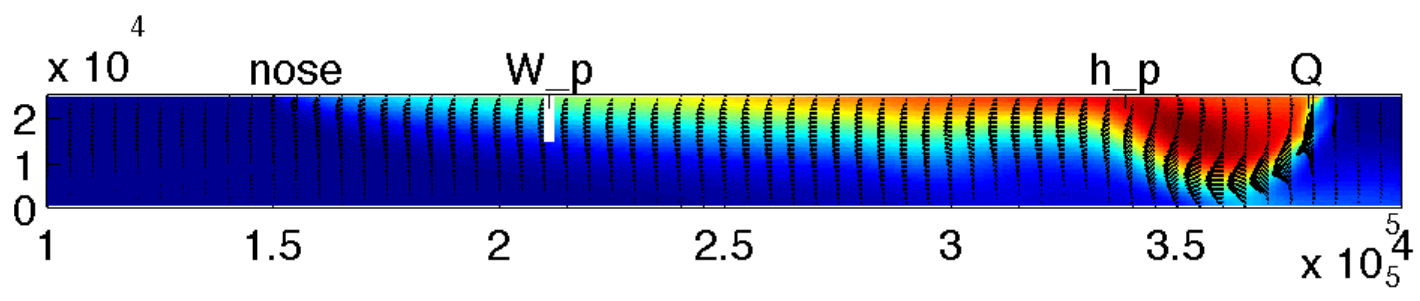

Figure 3-7: Plume scales. Distances (x and y axes) are in meters.

density $\rho_{0}$ in equations $3.1,3.2$ and 3.3 to be the layer 2 density, i.e. the density of the thermocline layer. In making the comparison to the simple theory, we are testing whether having a finite middle layer in the model makes a difference for the plume scales that come out of the model.

A single value of $h_{p}, W_{p}$, and $c_{p}$ is plotted for each run versus its theoretical value. For each model run, $h_{p}$ is given as the time-average of the maximum value of the depth of layer 1 along the coastal wall at each time step, $W_{p}$ is diagnosed as the distance from the coastal wall to the edge of the plume at a distance $\frac{3}{4}$ of the way between the river input and the nose of the plume, and $c_{p}$ is taken to be the speed at the very front edge of the deepening plume propagates down-coast.

For the plume depth, nose propagation speed, and plume width, the correlation coefficient between the model and theory is .89, .91, and .31 (p-values $<<.0001$ for $h_{p}$ and $c_{p}$, p-value $=.05$ for $W_{p}$ ), respectively (see figure 3-8). Despite the high correlation, the theory overestimates $h_{p}$ and $c_{p}$ while underestimating $W_{p}$. The finite-depth intermediate layer in the model does respond to the deepening of the plume layer, and there is flow in this layer which may sap some energy from the plume layer. While the details of the 2.5 layer system may differ from the 1.5 layer system, we nonetheless continue with the model as it is in fairly good agreement with our theoretical 

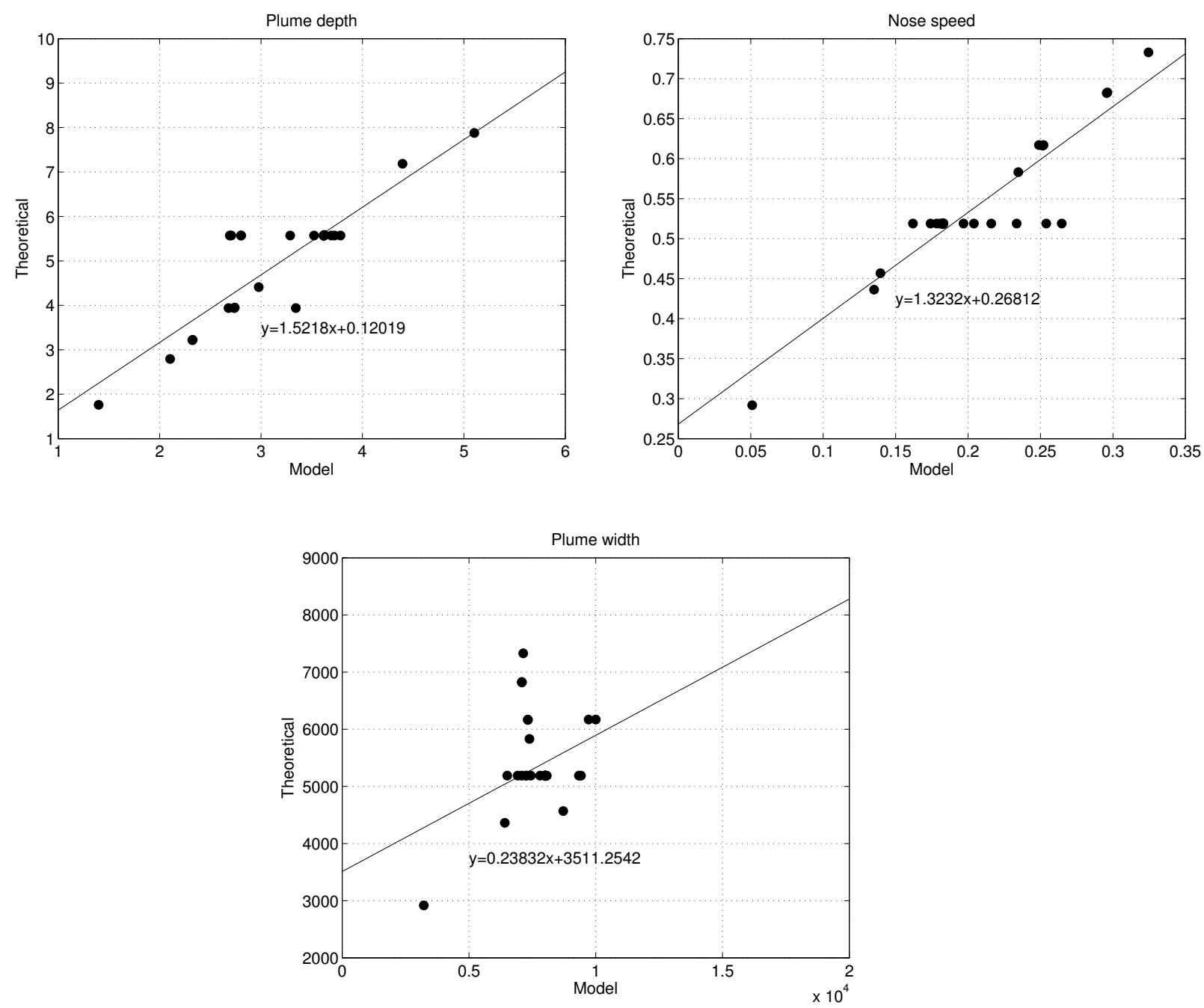

Figure 3-8: Plume depth, width, and propagation speed, model vs. theory

understanding of buoyant coastal plumes.

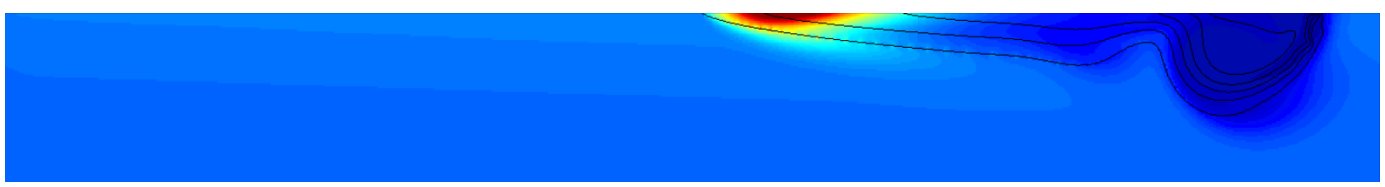

Figure 3-9: Snapshot of copepod concentration (colors) and plume layer depth (contours) from an example model run (same model run and axes as figure 3-7). Colors range from 0 to 6 .

The tracer flux into the model through the rivers is zero, so we can test how well the model conserves tracer relative to the other fields (for instance, volume of a 
specific layer). As the plume develops and moves down the coastline, concentration increases at the plume nose due to stretching of the plume layer beyond the target depth of the copepods. While the volume of the plume layer increases, the total amount of copepods in the domain should remain fixed, to within the precision of the model. To test this, we compare the percentage change of layer 2 volume to that of copepod concentration. Figure 3-10 indicates that the percentage change of layer 2 volume and copepod concentration integrated over the domain are nearly identical, and are much less that $1 \%$. Thus, we can conclude that the model does a reasonable job of conserving these properties.

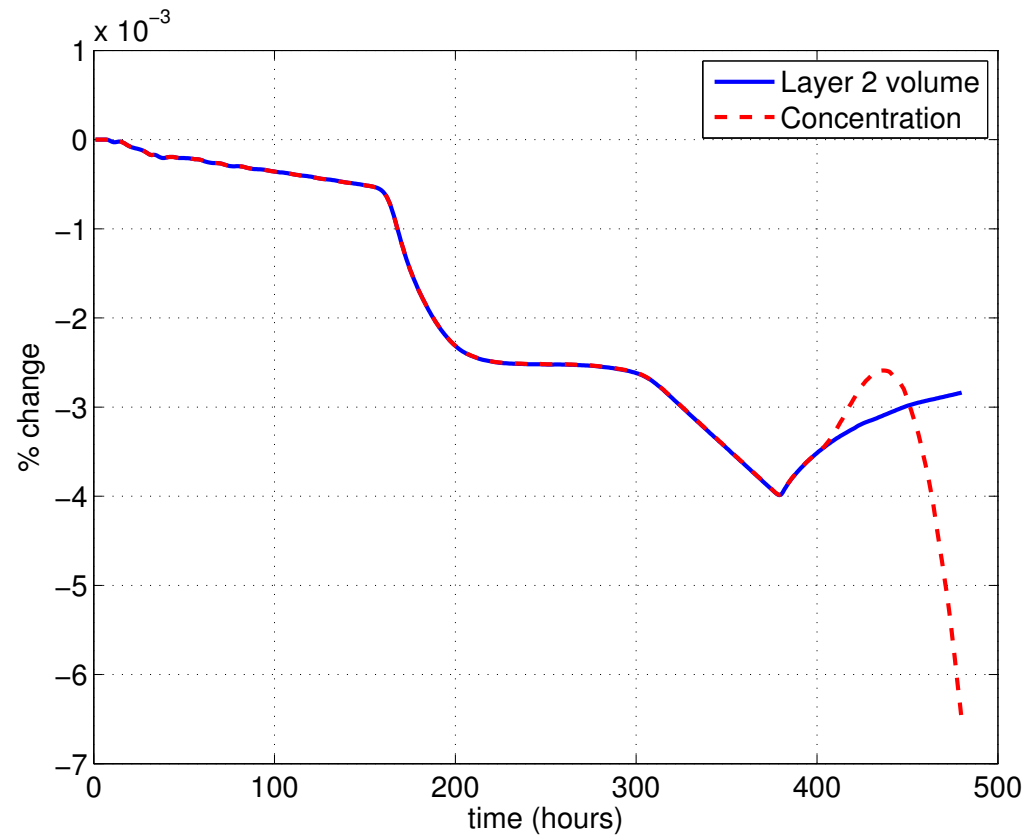

Figure 3-10: Percentage change of copepod concentration integrated over entire model domain, and layer 2 volume

\subsubsection{Plume-relative circulation}

The circulation relative to the moving plume determines how the copepods will move, and thus is pivotal to determining the final copepod distribution. In this section, we isolate the circulation relative to the plume in order to better understand how it will 
effect copepod distributions given different behaviors.

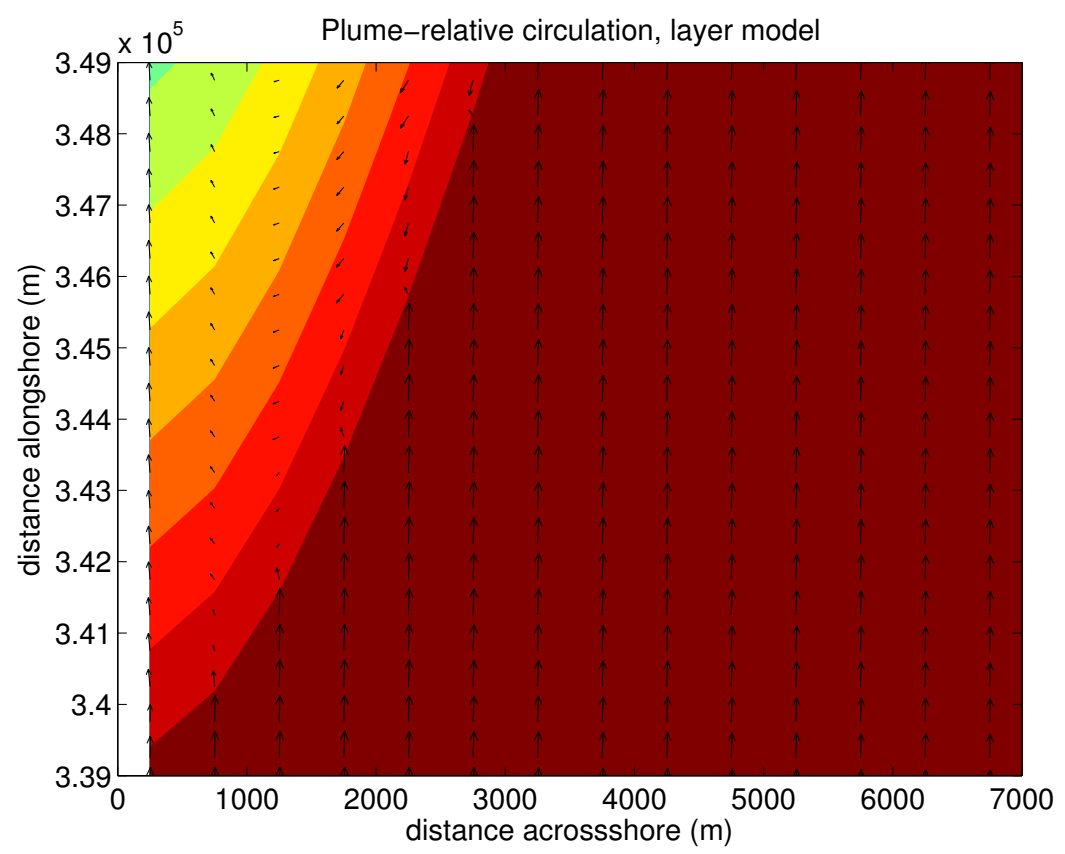

Figure 3-11: Circulation around nose of plume relative to plume (mean plume speed removed) for layer model. Colors are plume-layer thickness.

In the ROMS setup, the horizontal circulation relative to the moving nose of the plume is such that the interior fluid is deflected offshore and runs along the edge of the plume (figure 3-11). Within the plume, the flow is towards the nose along the wall, and away from the nose offshore. In this model, depth-keeping copepods will be deflected offshore as the plume moves down the coast. For the layer model, the sense of the plume-relative circulation is the opposite: along the wall the flow is away from the nose, while at the offshore of the edge the plume the flow is towards the nose. In this case, we may expect to find copepods at or near the intersection of the plume with the coastal wall.

The ROMS circulation is consistent with what we may expect from a plume propagating along a vertical coastal wall, while that of the layer model is more like what we'd expect from a plume propagating over a sloping bottom (see Lentz and Helfrich (2002)), although for a different reason. In the case of a coastal plume flowing over a sloping bottom, the plume-relative circulation results from entrainment along the 


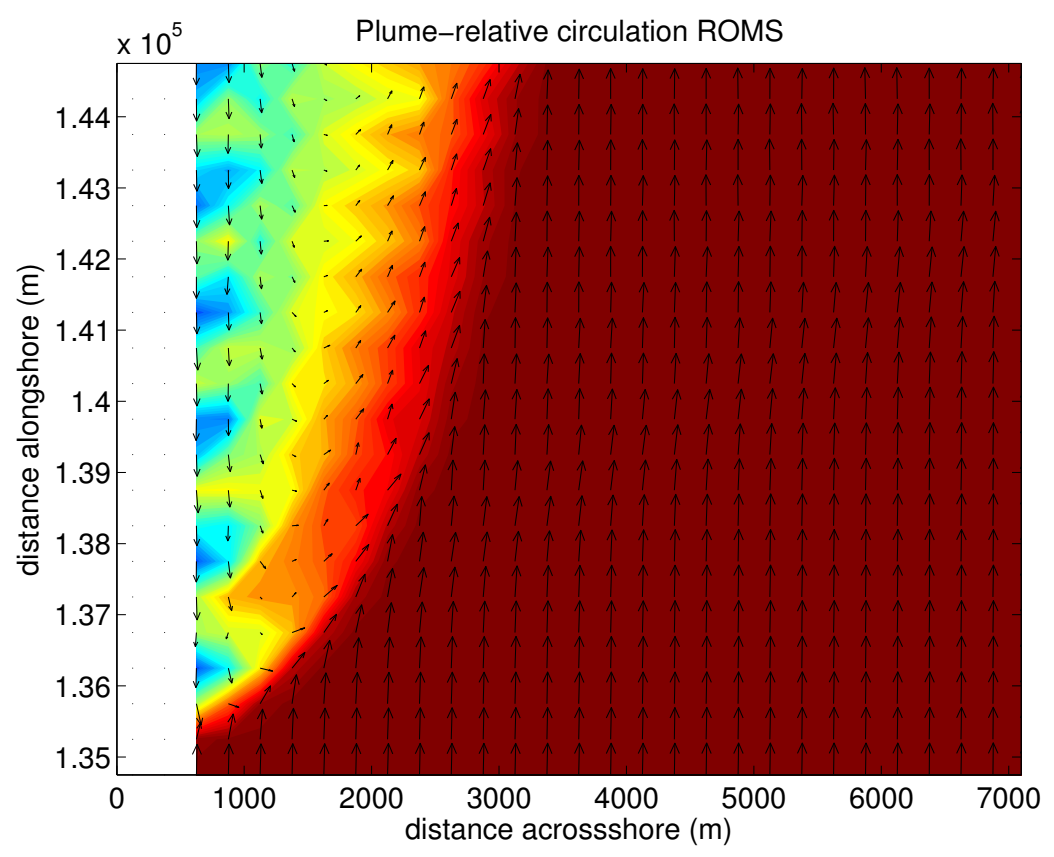

Figure 3-12: Circulation around nose of plume relative to plume (mean plume speed removed) for the ROMS plume. Colors are surface density

offshore edge of the plume. There is no entrainment in the layer model; the reason that the plume-relative circulation in ROMS and the layer model are in opposite directions is likely due to the effect of the coastal boundary condition. The boundary condition at the wall in the layer model is "no slip", meaning the flow in the along-shore direction must go to zero there. When we look at the flow in a reference frame moving with the nose, it appears as if the flow is moving away from the nose here. Switching the boundary condition to "free-slip" causes the along-shore flow to be maximum along the wall, and the resulting plume-relative flow reverses direction. The results that follow do not depend upon the boundary condition being "free-slip" or "no-slip", and thus we continue with the "no-slip" boundary condition.

In ROMS it is possible to look at the vertical distribution of circulation associated with the propagating nose. While the depth-keeping copepods will not move vertically, the isopycnal and passive copepods will. In a reference frame moving with the plume (figure 3-13) we can see that the interior water moves towards the nose and downward at the nose. This vertical motion extends downward to the thermocline, 

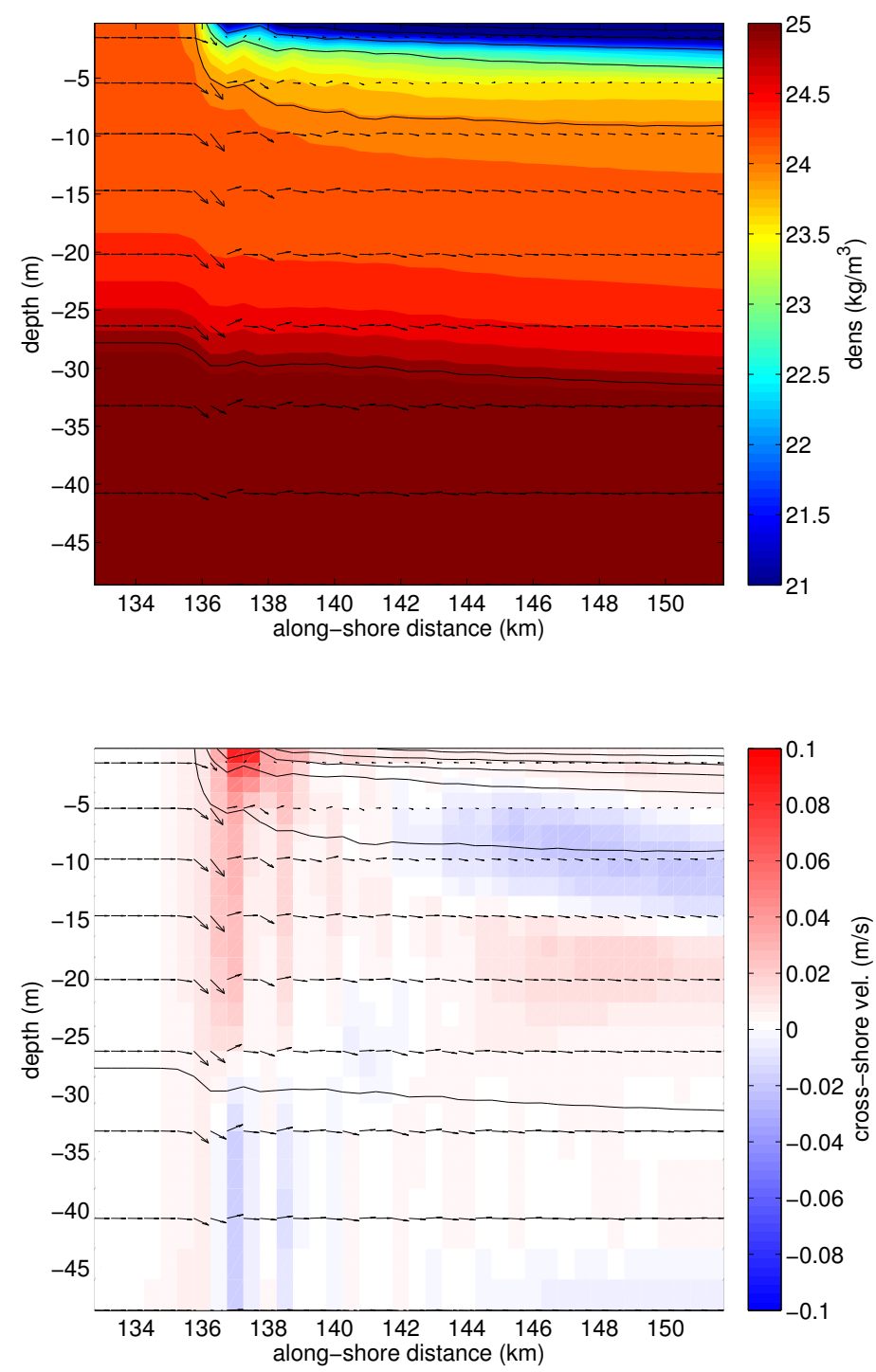

Figure 3-13: Vertical section of circulation around nose of plume relative to plume (mean plume speed removed) from ROMS.

where it begins to decay with depth. In this sense, even copepods that are sitting beneath the depth of the plume would feel the downwelling effects of the nose as it propagated overhead. This type of plume-relative motion is exactly the type of horizontally-convergent motion necessary for the coupled aggregation mechanism to work.

Helfrich and Pineda (2003) noted the importance of non-hydrostatic flow in the 
nose of a gravity current. While their work is in a non-rotating reference frame, we nonetheless compare the flow from ROMS in figure 3-13 (a hydrostatic model) with similar sections from Helfrich and Pineda (2003). First, we notice that the spatial scale of the gravity current in their model is much smaller than ours. They also have much higher grid resolution in the nose vicinity than we do in our model. ROMS does parameterize non-hydrostatic processes, but how well does this parameterization capture the non-hydrostatic nature of the nose? The important characteristics of the plume-relative flow in Helfrich and Pineda (2003) is that the flow is towards the nose (faster than the nose) at the surface, turns downward at the leading edge of the nose, and is away from the nose at the base of the plume. As a result of this flow, the plume is slightly deeper in the nose region than behind the nose. We see some similar hints of circulation in ROMS. The flow is actually slightly faster towards the nose at the surface and away from the nose at the base of the plume, and the plume at the nose appears to be slightly deeper than the plume just behind the nose. The differences in resolution, rotation, and the fact that Helfrich and Pineda (2003), like other studies, ignores three-dimensional flow, make a more-direct comparison between the two models difficult, but the good qualitative agreement between the two models implies that ROMS is capturing the important characteristics of the plume-relative flow.

The cross-shore motion, ignored in many studies such as Epstein and Beardsley (2001), may also turn out to have an important impact on the resulting copepod distribution. In the ROMS model run, at the plume nose there is flow in the offshore direction, which is strongest near the surface but extends down to the thermocline (approximately $25 \mathrm{~m}$ depth) (right hand panel of figure 3-13, red is out of page). About $10 \mathrm{~km}$ behind the nose of the plume, in the waters between the plume and the top of the thermocline, we see an interesting two-layer flow appear; the water just under the plume is flowing towards the coast, while the flow above the thermocline is flowing away from the coast.

Figure 3-14 shows a cross-shore section of density and velocity taken approxi- 
mately $10 \mathrm{~km}$ behind the plume nose. Here, we see the cross-shore flow described above appear as a recirculating flow towards the coast just beneath the plume, downward along the base of the plume and at the coastline, then turning offshore at the top of the thermocline, and finally upwelling near the offshore edge of the plume.
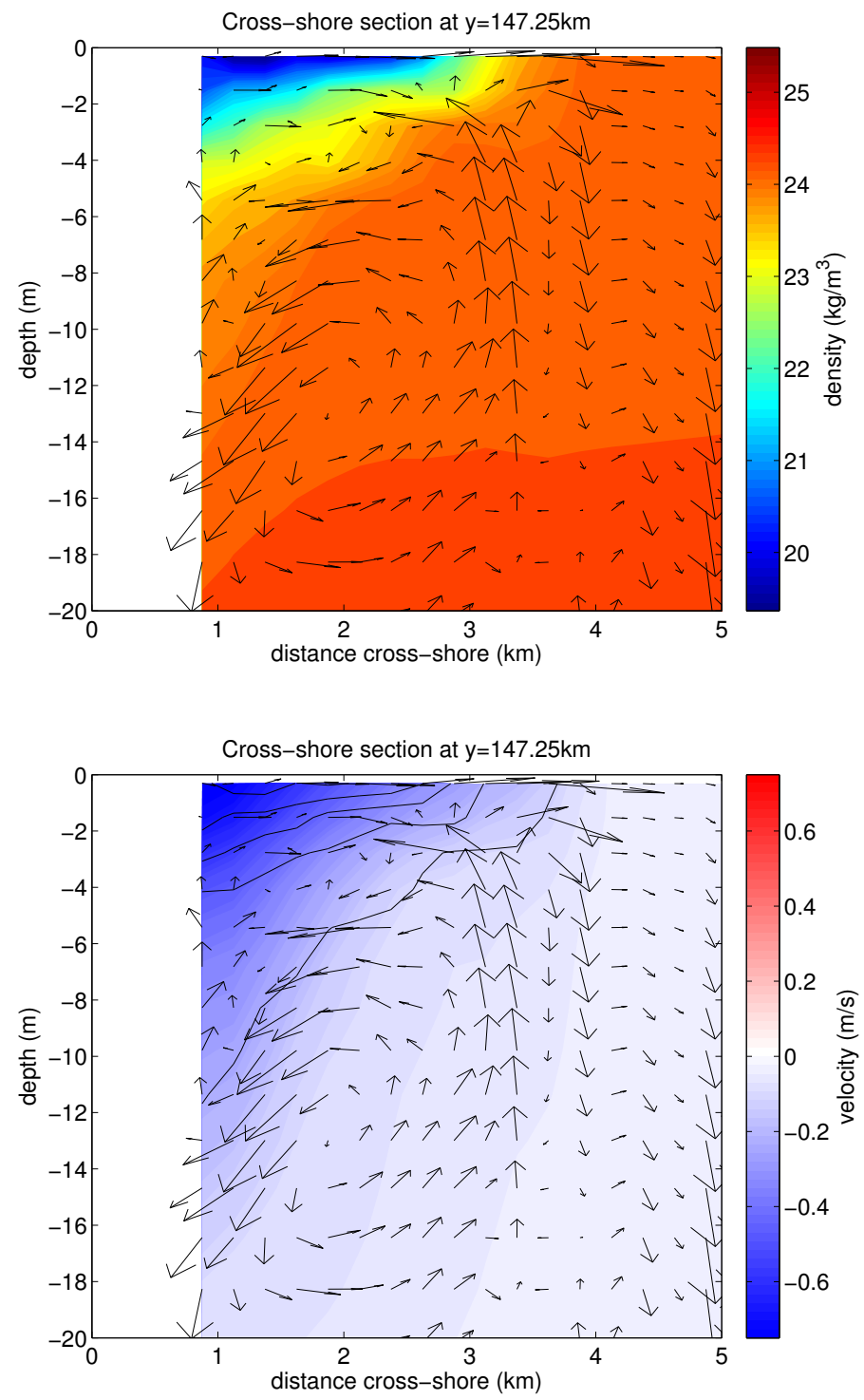

Figure 3-14: Vertical section of cross-shore circulation behind nose of plume relative to plume from ROMS.

The nature of this flow is similar to ageostrophic circulation often associated with fronts (Niiler (2009)). Plotted in figure 3-15 are profiles showing the magnitude of 
different terms in the momentum equations just behind the nose. We can use these profiles to try and understand the dynamics of this overturning circulation. It is well known that the along-shore flow in the plume is geostrophically balanced by an acrossshore pressure gradient, which we can see in the cross-shore momentum terms (Lentz (2004)). What is less clear is what the balance is in the along-shore direction. None of the terms in the momentum equations are particularly dominant here, but what is clear is that the flow is not geostrophic. At the depths of the recirculation (roundly $5 \mathrm{~m}$ to $15 \mathrm{~m}$ ), the horizontal and vertical advection terms are largest in magnitude, and are largely balanced by one another.

At this point, we can also test our assumption of the copepods as "perfect swimmers". For the depth-keeping copepods, we have assumed that the copepods are "perfect swimmers", in that they can swim faster than the fastest vertical flow, which allows them to stay exactly at their target depth. Typical copepod swimming speeds are near $1-10 \mathrm{~cm} / \mathrm{s}$. The downwelling in ROMS is roughly $0.008 \mathrm{~m} / \mathrm{s}$, which is less than what we expect are typical copepod swimming speeds (although not much less).

A similar test can be executed in the layer model. While there is no explicit vertical velocity in the layer model, we can estimate what that vertical velocity would be using different methods. The first uses the plume propagation speed and plume nose slope, which combined will tell us how quickly fluid is driven downward against the plume nose. The slope of the plume nose is much smaller in the layer model than in ROMS, and thus we get a scale for the downwelling velocity of roughly $1 \times 10^{-4} \mathrm{~m} / \mathrm{s}$. The second method we can use is to calculate $\frac{\partial h}{\partial t}$ for the upper layer, which is analogous to $w$ for this model. The maximum value of $\frac{\partial h}{\partial t}$ for a typical layer model run is approximately $1 \times 10^{-4} \mathrm{~m} / \mathrm{s}$. The velocities in the layer model are much smaller than the typical copepod swimming speed, so in this model the assumption of "perfect swimmers" is not bad. 


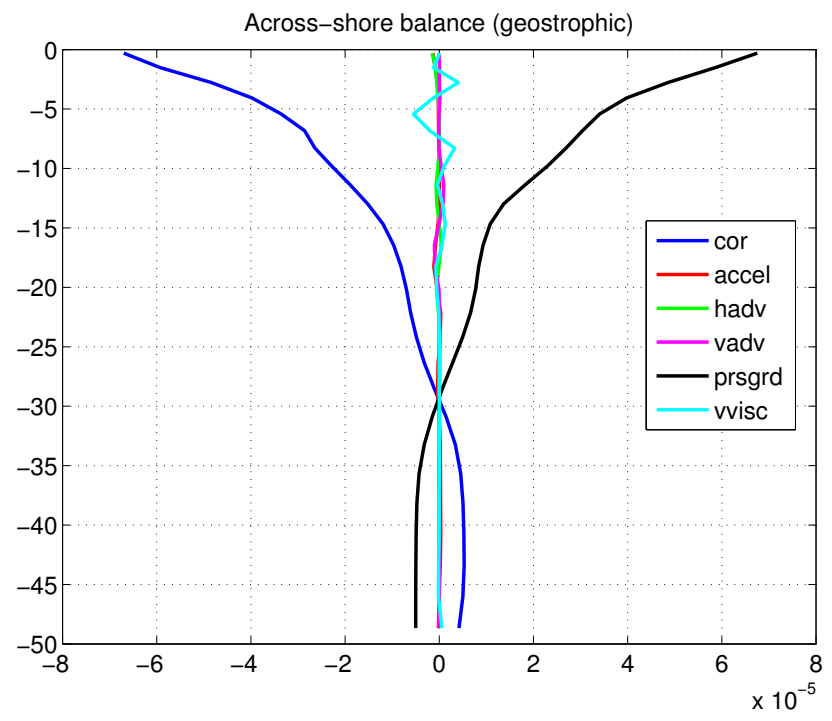

(a) Across-shore balance

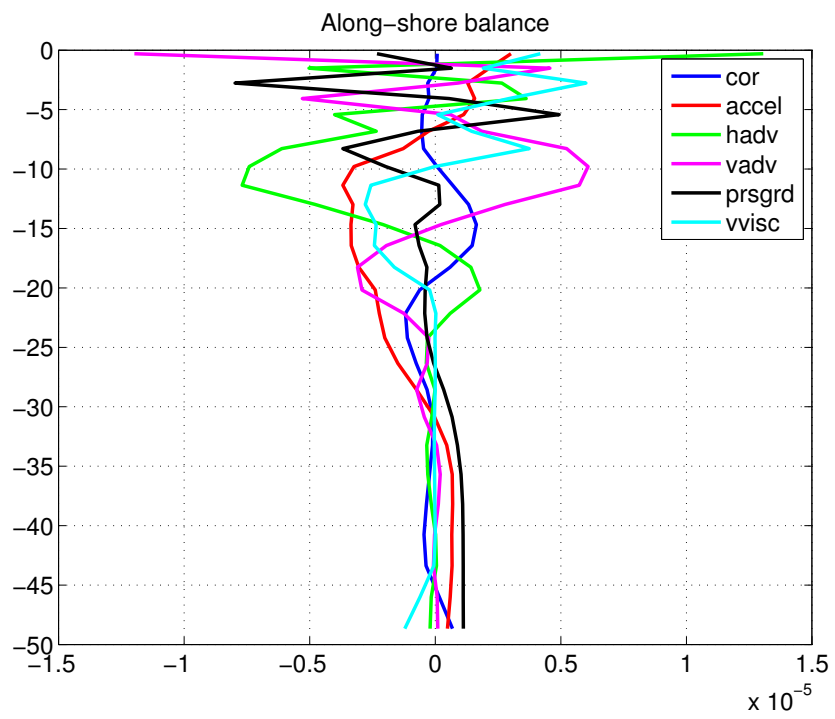

(b) Along-shore balance

Figure 3-15: Vertical profiles of terms in the momentum equations behind the nose of the plume.

\subsubsection{Layer model - how does the plume affect the resulting copepod distribution?}

Since the layer model coupled to the copepod concentration model reproduces a plume consistent with simple theory (section 3.4.1), it can be used to evaluate how the 
characteristics of a river plume affect the distribution of copepods within it. In the following many parameters of the idealized plume are changed:

- Upper layer (plume) density anomaly $\rho_{p}$

- Middle layer (mixed layer/thermocline) density anomaly $\rho_{2}$

- Diffusivity $\kappa$

- Copepod depth $d_{b i o}$

- River discharge $Q$

- Thermocline depth

Changing these different parameters have the effect of changing the plume depth $h_{p}$, nose propagation speed $c_{p}$, and width of the plume (see equations 3.1, 3.2, and 3.3), which can thereby affect the resulting copepod distribution.

The effects of the different parameters above on the maximum tracer concentration achieved by each model run can be understood by a simple empirical parameter, $S$ :

$$
S=\sqrt{\frac{h_{p}}{d_{b i o}} \frac{W_{p} c_{p}}{\kappa}}
$$

Figure 3-16 shows the maximum concentration for each model run as a function of S. Almost $90 \%$ of the variance in the maximum concentration is explained by this parameter, and the two are highly correlated.

The various parameters in the scale factor $S$ can be summarized as an advectivediffusive balance modified by the relative depths of the plume and copepod target depth. The advective-diffusive balance, shown by the ratio $\frac{W_{p} c_{p}}{\kappa}$ indicates that copepod concentration increases at the nose due to copepods being swept up by the plume, and is tempered by diffusion of copepods away from this location. Although the biological target depth $d_{b i o}$ is a somewhat crude biological parameter in that it assumes "perfect" swimming by the organisms, it affects the resulting distribution in interesting ways. For a given plume, the smaller $d_{b i o}$, the closer the organisms are to the 


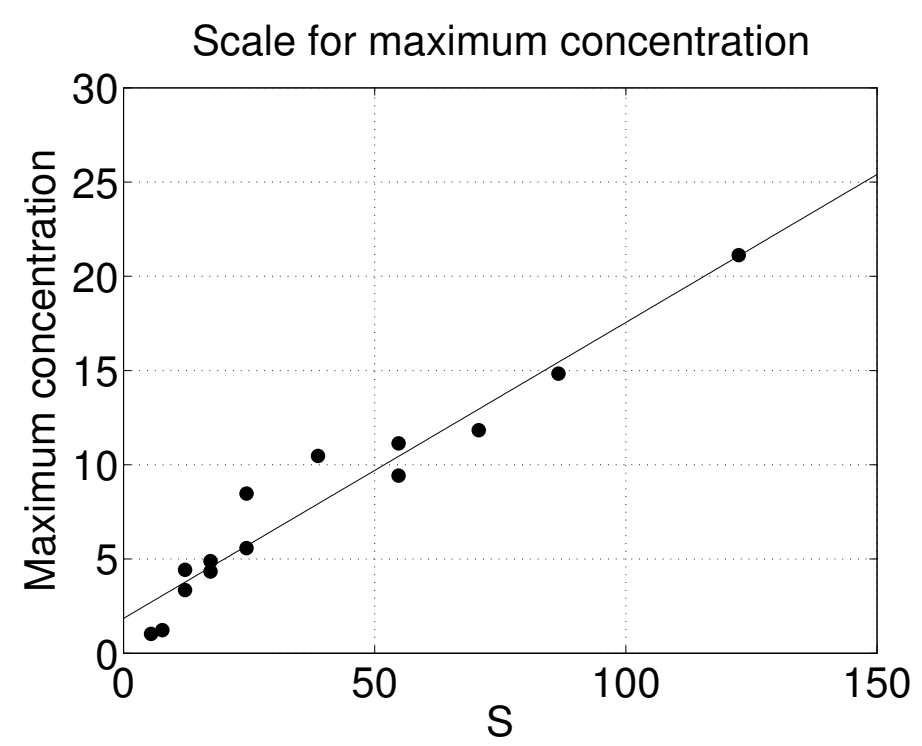

Figure 3-16: Maximum concentration in a given model run as a function of nondimensional parameter $S$

surface, and a higher concentration results. Similarly, for a given biological target depth, deeper plumes result in higher copepod concentrations. This is because the copepod concentration increase is proportional to the water-column stretching at the copepod depth, which depends upon how deep the plume stretches beyond the depth of the copepods.

We can also develop a scale for the area of the patch that results from the model run. In order to normalize patch area over many runs with different maximum concentrations, we define the area of a patch as the total area in the model where the concentration is greater than $\frac{1}{3}$ of the largest concentration in the model.

$$
S_{a}=\sqrt{\frac{d_{b i o} \kappa}{W_{p}^{2} h_{p} f}}=\sqrt{\frac{d_{b i o}}{h_{p}} \frac{\kappa f}{c_{p}^{2}}}=\frac{1}{S}
$$

The factor $S_{a}$ is the inverse of $S$; larger patch area (as defined) will result when diffusion outweighs advection due to the motion of the plume. The ratio between $d_{b i o}$ and $h_{p}$ appears again in equation 3.5, but is reversed from that in equation 3.4. $\kappa$ appears in 3.5 in a way that is not surprising. Diffusion acts to spread a patch 


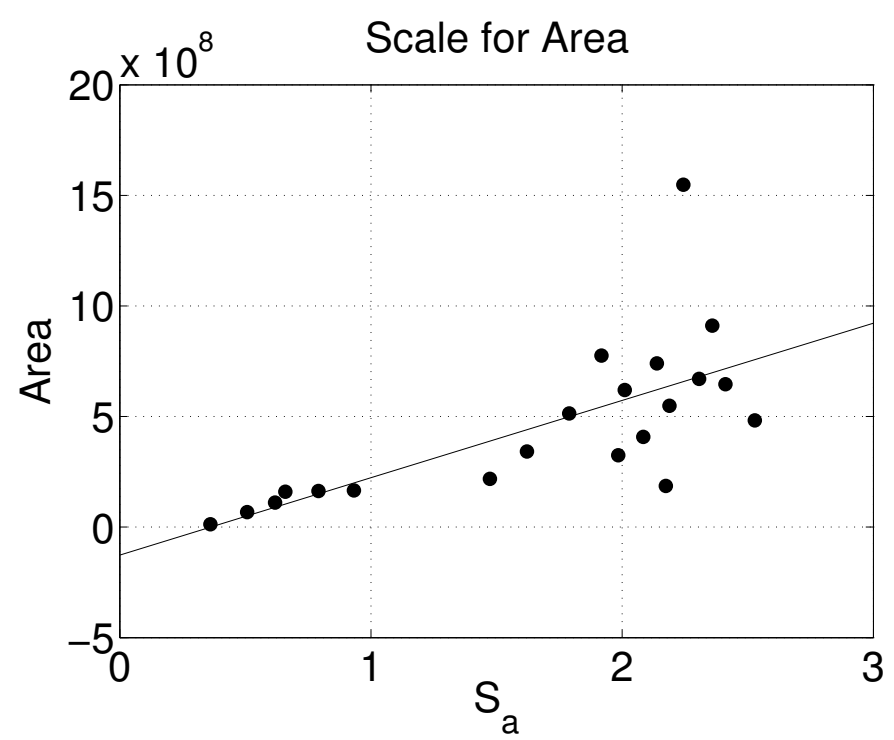

Figure 3-17: Patch area in a given model run as a function of nondimensional parameter $S_{a}$

out, thus for runs with higher diffusion the patch is more spread, resulting in a larger patch area. Plume width and plume nose speed both appear in the denominator of $S_{a}$ because they approximate the scale of the advection.

\subsubsection{ROMS/LTRANS}

Drifters are deployed at different depths throughout the model, but in the following discussion we divide these drifters into two groups: those deployed near the surface and those deployed near the top of the thermocline. It is not clear from observations whether actual copepods prefer to be just at the surface, or somewhere in the mixed layer, or at the base of the mixed layer, and so we differentiate between these parts of the surface waters in order to discern how the different behaviors determine the resulting copepod distribution. We begin with drifters initially deployed at the surface. 


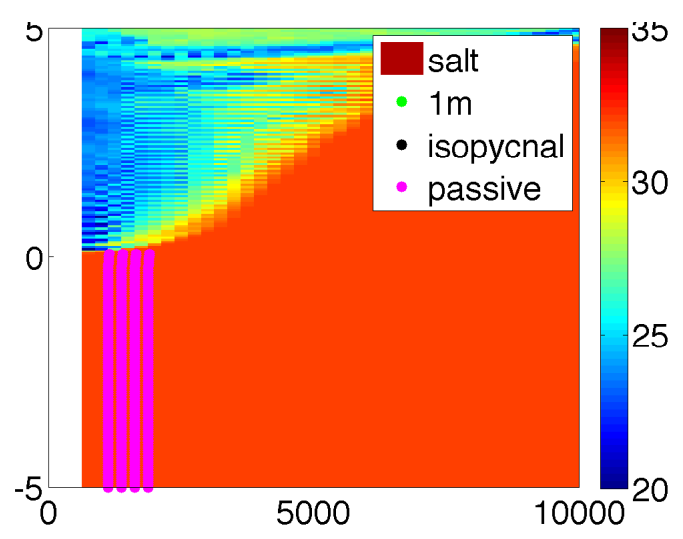

(a) $t=44$ hours

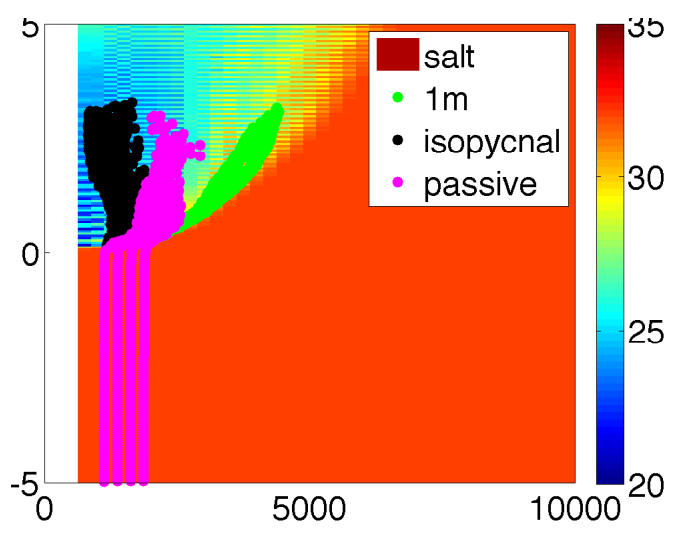

(c) $\mathrm{t}=68$ hours

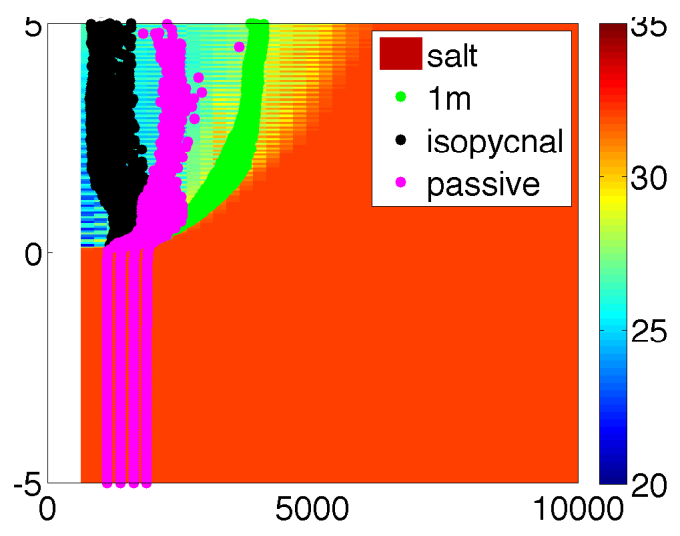

(e) $t=92$ hours

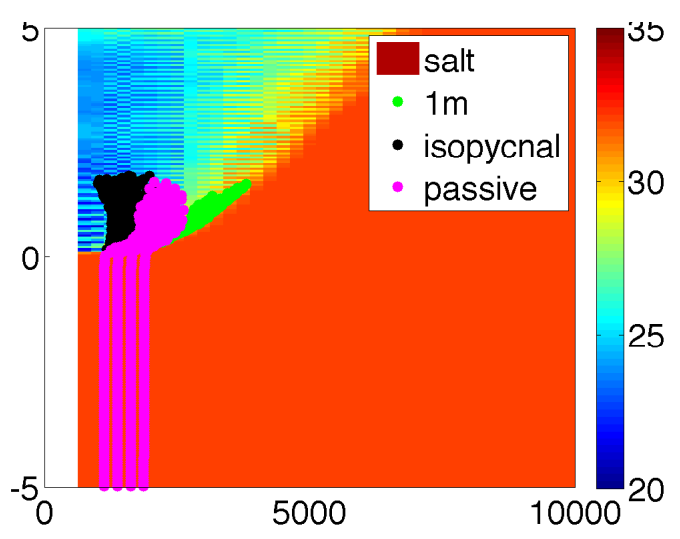

(b) $\mathrm{t}=56$ hours

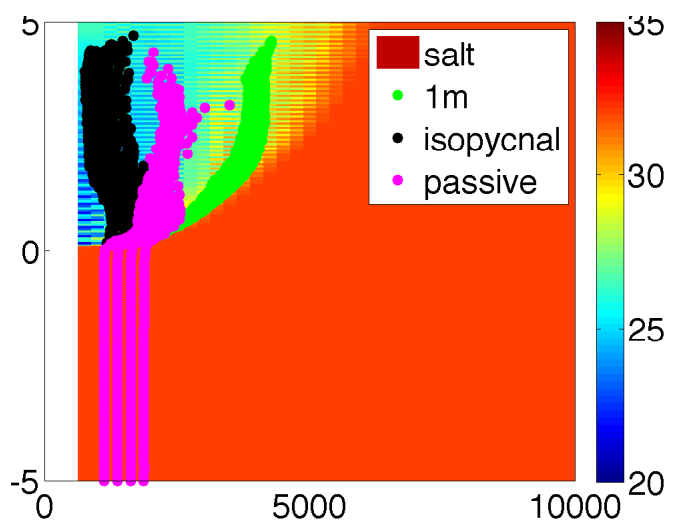

(d) $t=80$ hours

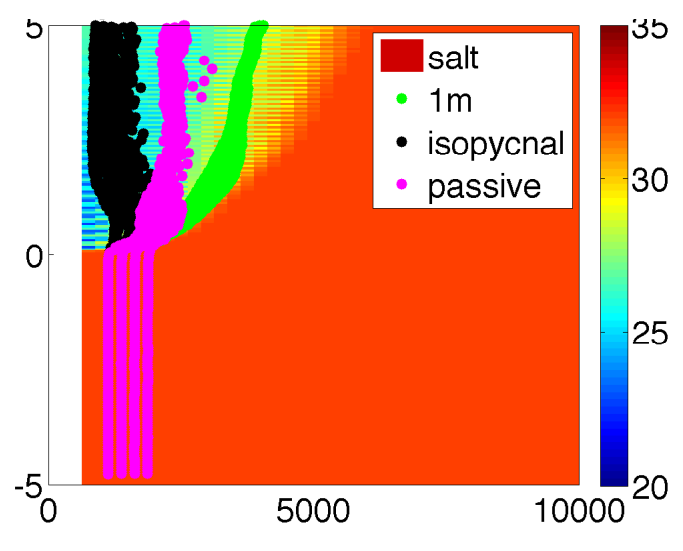

(f) $t=104$ hours

Figure 3-18: Horizontal maps showing the distribution of surface drifters at various times during the plume's propagation through the drifter array. Snapshots taken in a reference frame moving with the plume nose. $\mathrm{x}$ and $\mathrm{y}$ axes are distances in meters. 


\section{Surface drifters}

Figure 3-18 shows the lateral distribution of the three different drifter types deployed at $1 \mathrm{~m}$ while the nose of the plume propagates through the drifter array. The depthkeeping drifters are advected alongshore by the plume (as we might expect from the theoretical mechanism), but are also deflected offshore by the plume. The passive drifters also deflect offshore, but not to the same extent as the depth-seeking drifters. It is not apparent in these images, but these drifters actually sink beneath the plume in addition to being advected offshore. The isopycnal drifters are initially pushed offshore when they first contact the nose of the plume (and also sink as the passive drifters do), but after the passage of the nose, the isopycnal drifters actually move back towards the coastal wall, ending up closer to the wall than they had started.

The pathways taken by the drifters deployed at the surface also vary in the vertical. Figure 3-19 shows the along-plume distribution of the three drifter types. As the plume first contacts the drifter blocks (around hour 44), we notice the drifters at the leading edge of the cube are compressed towards each other, and the isopycnal and passive drifters are pushed downward. The isopycnal drifters are driven downward deeper than the passive drifters because they are constrained to remain at the same density surface; the passive drifters are following water parcels that get mixed with lighter water, thus causing the density of that parcel to change (and thus ends up at a shallower depth than the isopycnal drifters). This difference in vertical position matters for the amount to which these drifters are deflected in the across-shore direction. As we can see, the depth-keeping drifters remain in the surface layer, which is always moving offshore along the edge of the plume. Thus, these drifters continue to move farther and farther away from shore. The passive drifters sink to a level where there is very little cross-shore flow, and so after the passage of the nose they do not move in the cross-shore direction much. The isopycnal drifters are driven down the farthest, and thus end up in water moving back towards the coast.

The cross-shore distribution of drifters is also enlightening (figure 3-20). Again, we can see that the isopycnal drifters are driven much deeper than the passive drifters. 

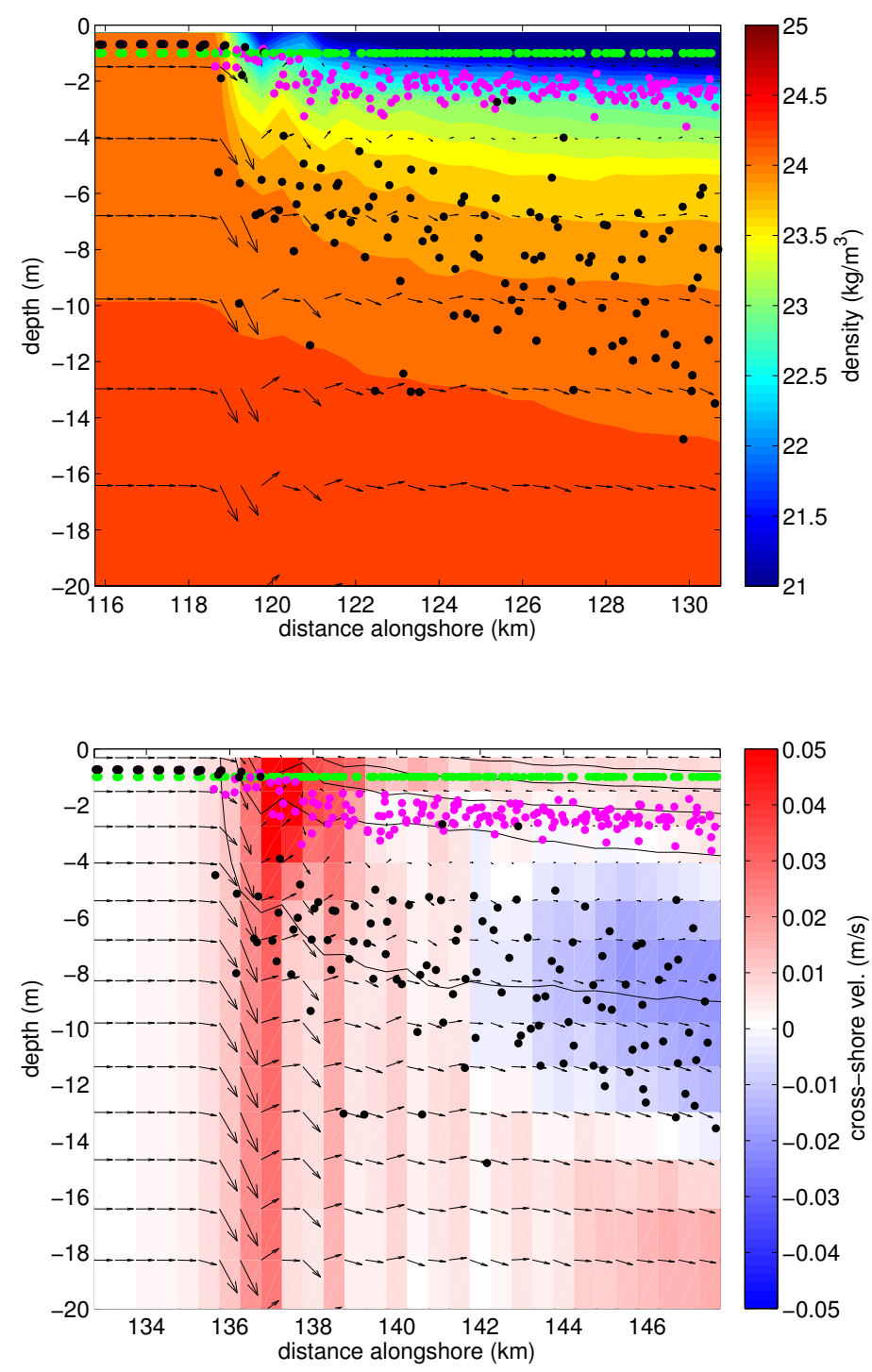

Figure 3-19: Vertical section (1km offshore) of circulation around nose of plume relative to plume (mean plume speed removed)

We can also see where these drifters fall in relation to the cross-shore circulation cell noted in a previous section. The passive drifters sit just above this cell, and are mostly unaffected by it. The isopycnal drifters, however, experience the effect of the cell, and move towards the coast and downward. As the flow turns offshore at the top of the thermocline, however, these drifters are left behind since they are inhibited in their vertical movement by the thermocline. 

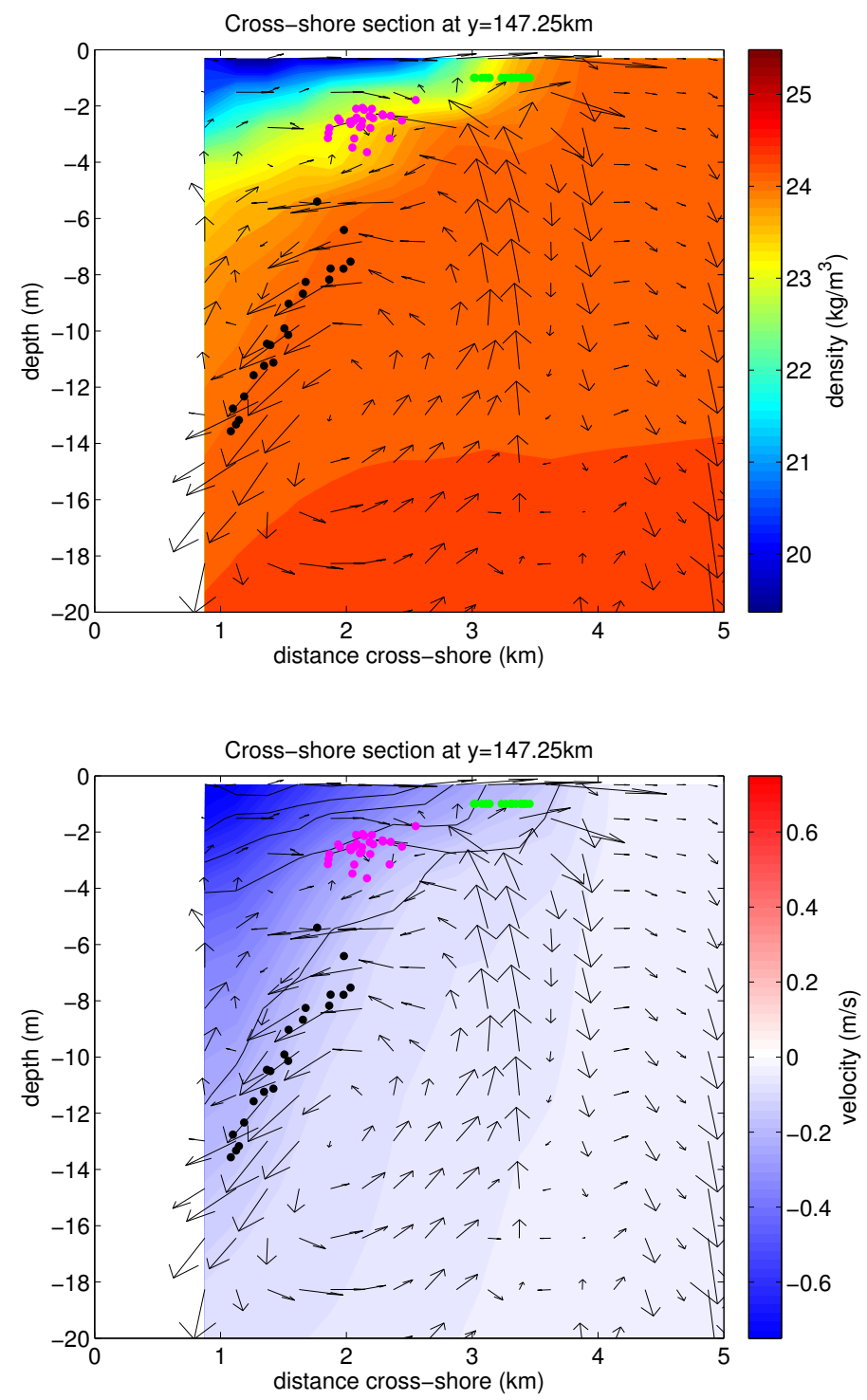

Figure 3-20: Vertical section of density and velocity taken approximately $10 \mathrm{~km}$ behind the plume nose

\section{Thermocline drifters}

Drifters released at depths between $10 \mathrm{~m}$ and $15 \mathrm{~m}$ are deeper than the plume, and so may behave differently from those deployed just beneath the surface. In this section we explore how these drifters move relative to one another.

Figure 3-21 shows the horizontal distribution of the three different drifter behaviors. It is apparent that there isn't a lot of difference between the different drifters: 
All three drifter types are slightly deflected offshore at the nose, then spread out behind the nose. Horizontally, there is no appreciable difference between these drifters. It is only in the vertical in which we see the difference.

Figure 3-22 shows the distribution of the thermocline drifters in the along-plume direction. Here, we can see that the depth-keeping drifters remain at their release depths. The passive drifters spread out in the vertical: some move upward, while others move downward to deeper water. The isopycnal drifters, however, appear to uniformly move downward by about $1-2 \mathrm{~m}$. The depth range of the drifters plotted encompasses the depths of the cross-shore circulation cell mentioned in previous sections, which explains why some of the drifters move shoreward while others move offshore.

Figure 3-23 shows the distribution of drifters deployed at the thermocline with respect to their cross-shore position $10 \mathrm{~km}$ behind the nose of the plume. Again, the passive drifters are more spread out in the vertical, while the isopycnal drifters are mostly deeper than their release depth. We can also see from this that the drifters here are released at a depth near the cross-shore recirculation. In particular, the isopycnal drifters in this snapshot appear to be similarly distributed compared to those drifters released at the surface (shown in figure 3-20). This illustrates the fact that the isopycnal drifters are compressed in the vertical (the surface drifters end up at the same depth as those drifters deployed at 10-15m), which will be important in the following section.

\section{Drifter diagnostics}

We have qualitatively explained the differences between the different drifters, but now we attempt to quantify these differences, and what they might mean for copepod distributions in the wild. The first diagnostic we'll define is the nearest-neighbor distance. To compute this metric, for every drifter we compute the distance between this drifter and its nearest neighbor. Plotted in figure 3-24 is the nearest neighbor distance averaged at the surface and at the thermocline. 


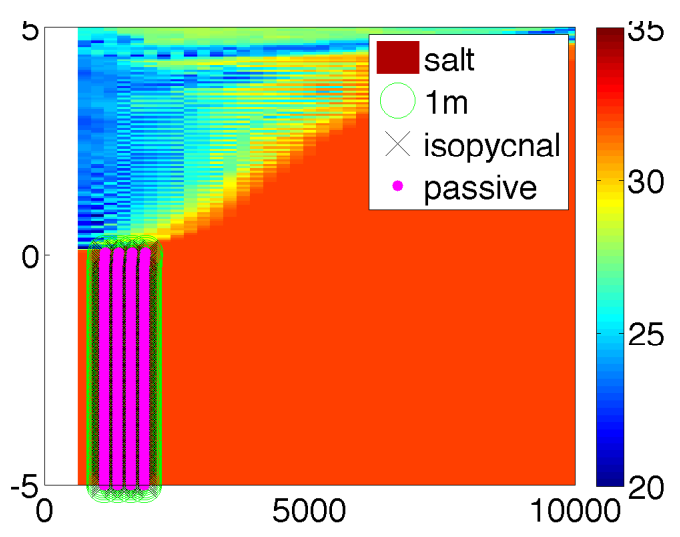

(a) $t=44$ hours

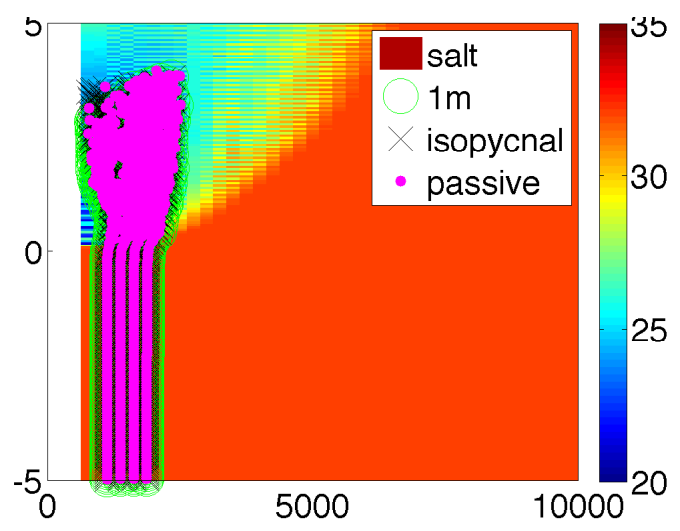

(c) $\mathrm{t}=68$ hours

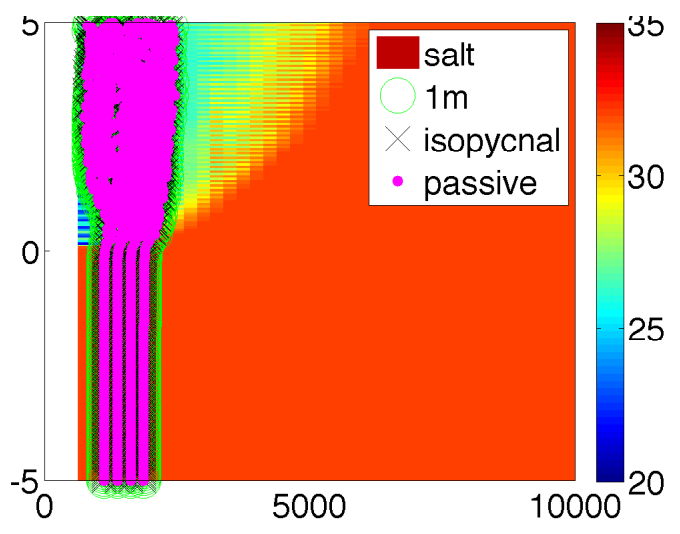

(e) $t=92$ hours

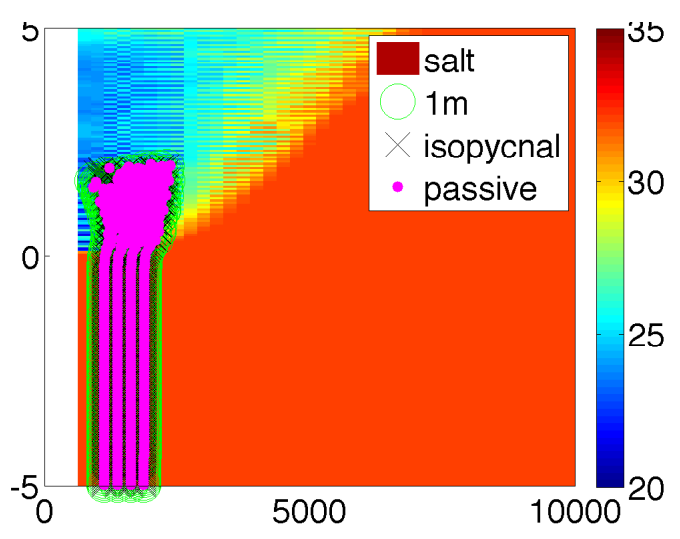

(b) $\mathrm{t}=56$ hours

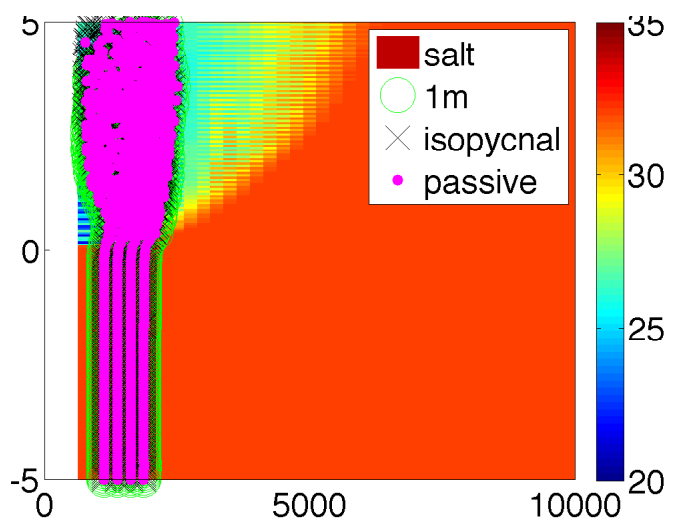

(d) $t=80$ hours

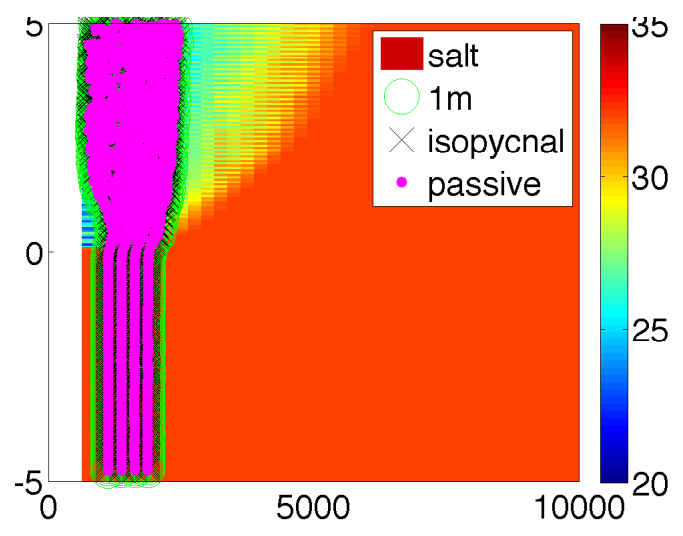

(f) $t=104$ hours

Figure 3-21: Horizontal maps showing the distribution of thermocline drifters at various times. Snapshots taken in a reference frame moving with the plume nose

For drifters deployed at the surface, the average distance to the nearest neighbor goes down, no matter what behavior the drifters exhibit. The depth-keeping drifters, 

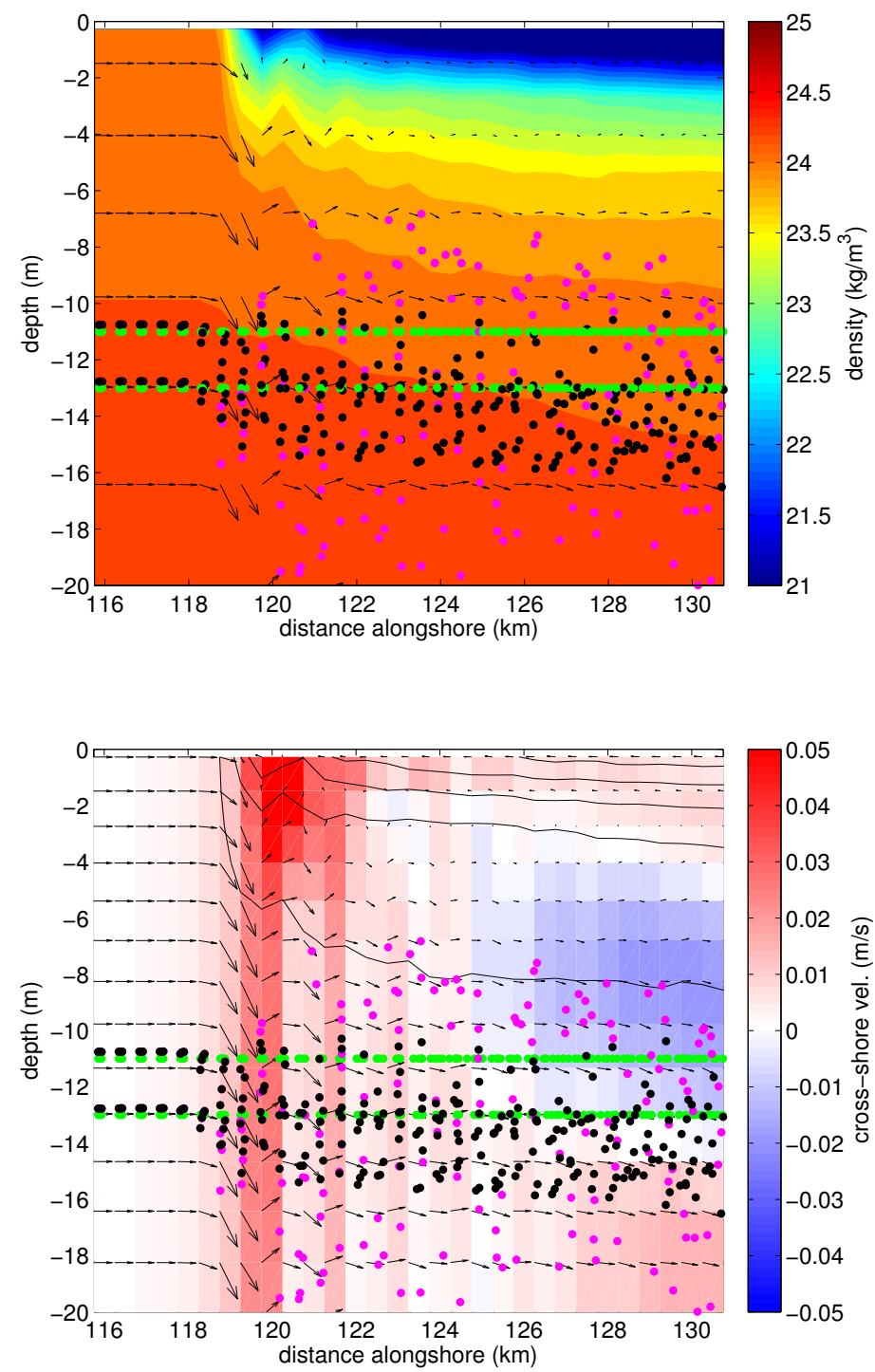

Figure 3-22: Vertical section of density and velocity taken approximately $10 \mathrm{~km}$ behind the plume nose.

however, become on average much closer to their neighbors than either of the other two behaviors. We saw in the previous sections that the isopycnal and passive drifters end up in very different places relative to the plume, however the average distance to their nearest neighbors is very similar, indeed perhaps are indiscernible from one another. This result suggests that copepods that are isopycnal-following will not end up closer on average to their nearest neighbor than a completely passive particle. 

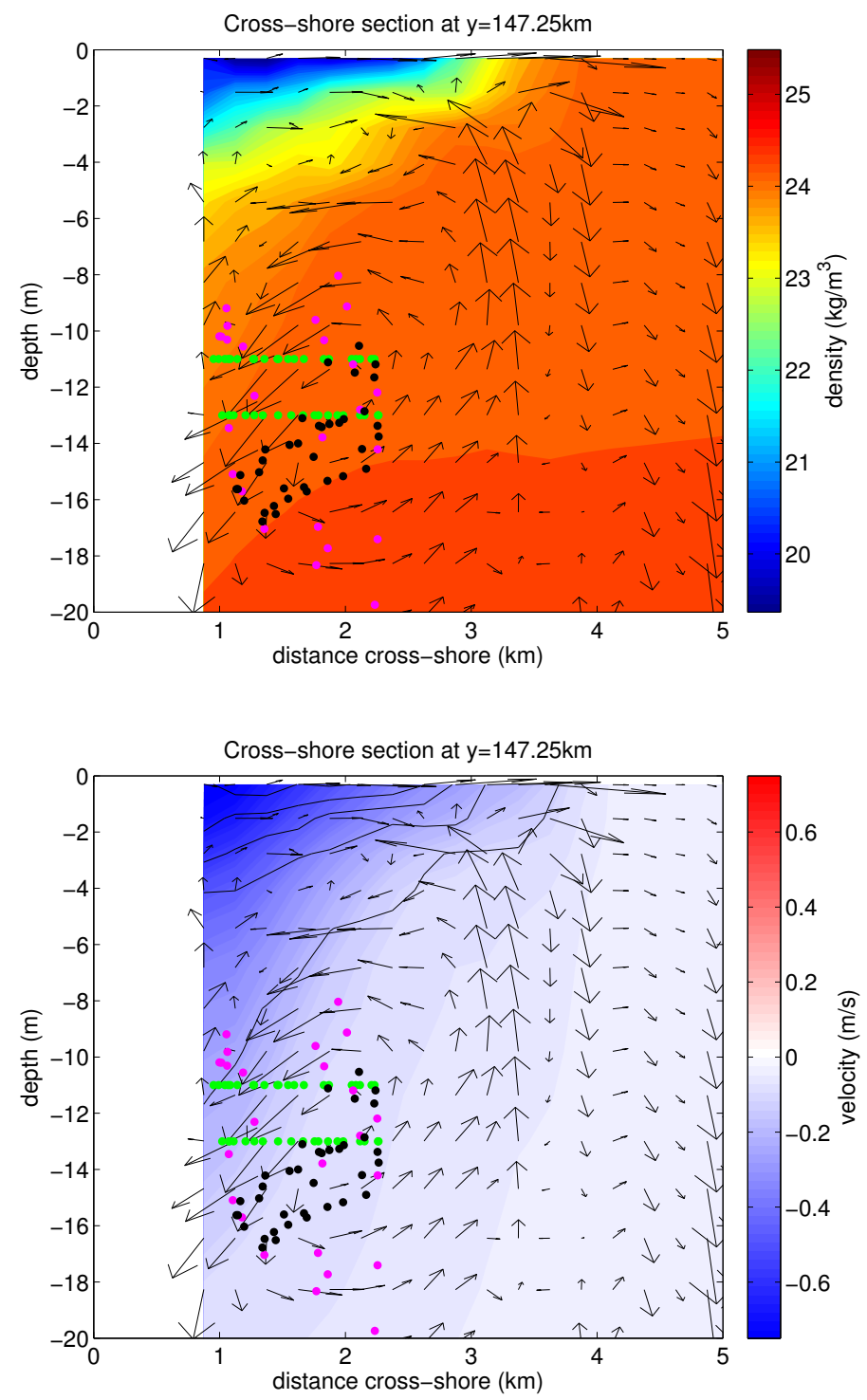

Figure 3-23: Vertical section of density and velocity taken approximately $10 \mathrm{~km}$ behind the plume nose

The nearest-neighbor distance for drifters deployed at the thermocline exhibit some different behavior than the surface drifters. First, the nearest-neighbor distances don't change nearly as much as they do at the surface. Also, the different behaviors are very similar: the nearest-neighbor distance decreases for each behavior by roughly the same small amount. It is apparent from this that the behavior exhibited in the thermocline does not matter as much for the resulting distance to the nearest neighbor 

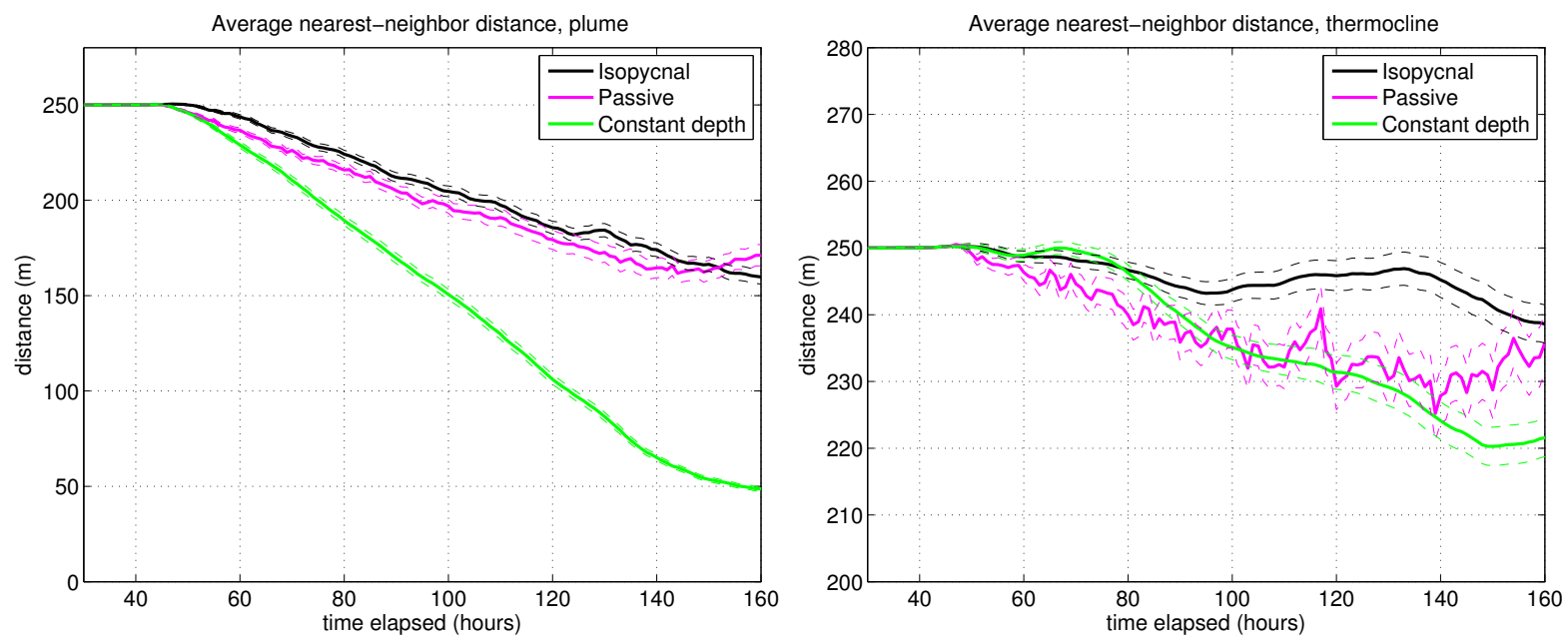

Figure 3-24: Average nearest-neighbor distance for surface and thermocline drifters. Note that the scales of the two plots are different.

as it does in the surface waters.

Another metric we can use to quantify the differences between the different types of drifters is a measure of the clustering of the particles. We compute clustering by counting the number of particles within $1 \mathrm{~km}$ horizontally and $4 \mathrm{~m}$ vertically of each particle, then averaging over all the particles in the domain. Again, we split the results into those drifters deployed near the surface and those deployed in the thermocline.

Figure 3-25 shows the average clustering for drifters deployed in the surface and thermocline, normalized by the initial clustering. For drifters deployed in the surface waters, the clustering increases with time. The passive and depth-keeping drifter clustering increases very similarly till about hour 90, after which time the depthkeeping clustering continues to increase while that of the passive drifters levels off. Interestingly, the increase is most drastic for the isopycnal drifters, which increase their clustering by more than a factor of two, larger than either the depth-keeping or passive drifters. The reason for this can be seen in figure 3-26. The isopycnal drifters deployed in the surface mixed layer all have the same initial density, despite being deployed over a wide range of depths. When the plume propagates along the coast, 

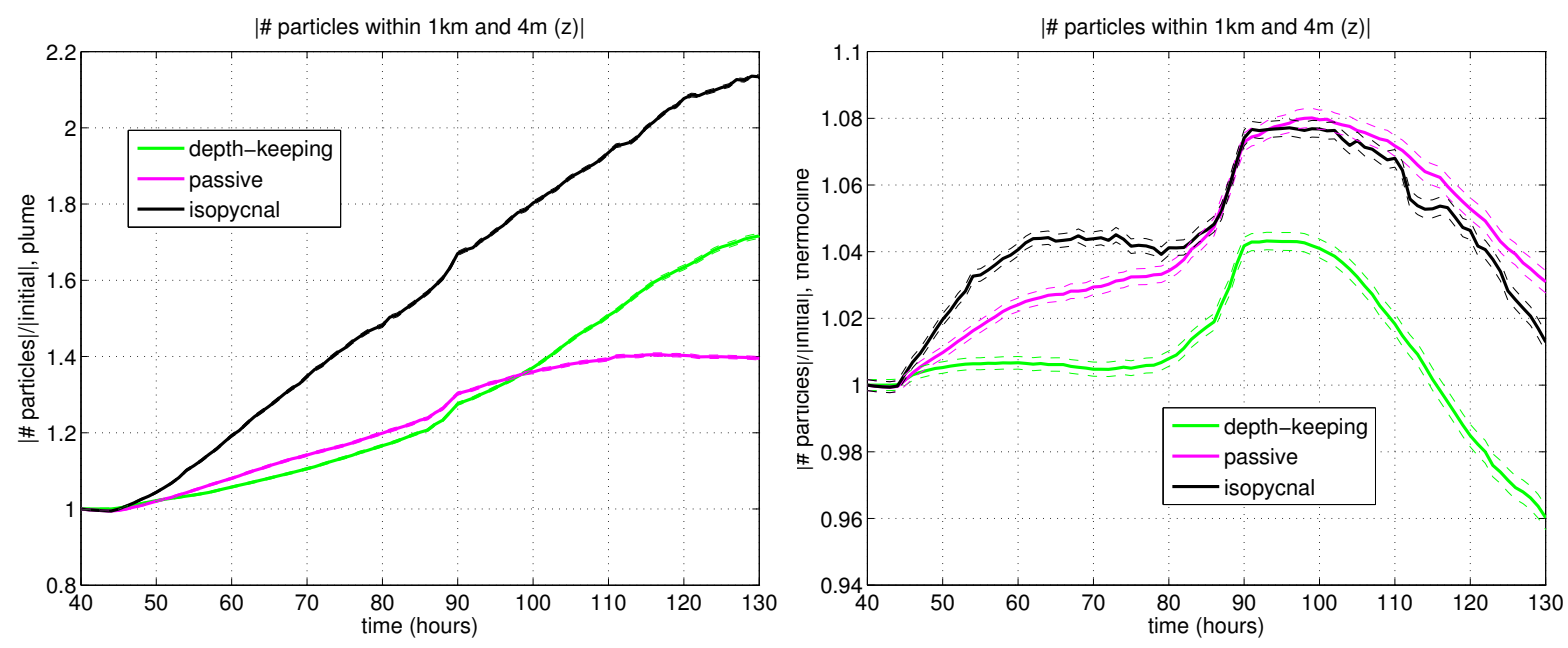

Figure 3-25: Average drifter clustering for surface and thermocline drifters. Note the different vertical scales on the two plots.

it compresses the surface mixed layer in the vertical. This means that the isopycnal drifters initially deployed over a wide range of depths ends up much closer together in the vertical.
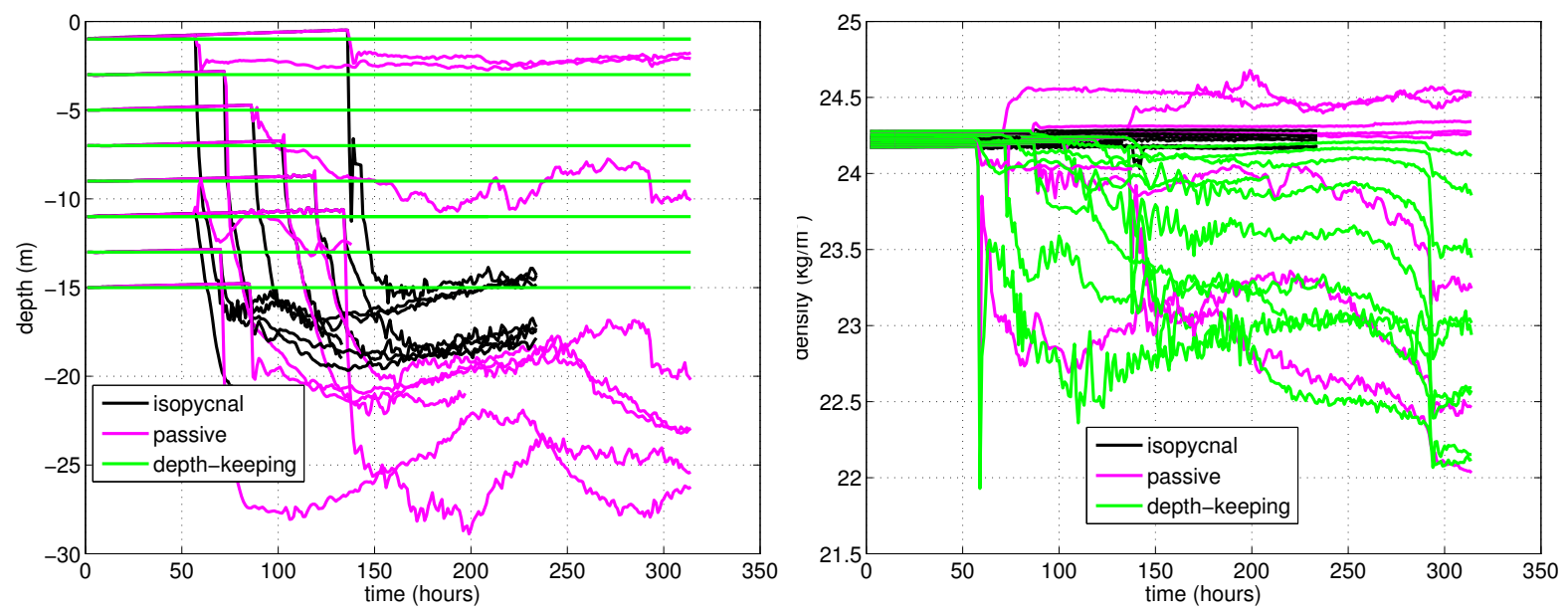

Figure 3-26: Particle depth (right) and water density (right) for several particles in ROMS.

Figure 3-26 shows the depths of the three different particle behaviors. Obviously, the depth-keeping particles stay at their deployment depths, while the other two types 
of drifters sink to deeper depths. The passive drifters remain more spread out in the vertical than the isopycnal drifters, which all end up at depths between about $15 \mathrm{~m}$ to $20 \mathrm{~m}$. This compression in the vertical explains why the clustering in figure 3-25 was so much higher for the isopycnal drifters than the other two drifter types.

The water density observed at the locations of the particles is also shown in figure 3-26. Since the depth-keeping drifters remain near the surface, the water density there decreases in time as a result of these drifters being advected into plume water. The passive drifters experience some mixing as they follow the 3-dimensional velocity field, and as a result some end up in lighter water while others end up in heavier water than what they started in. The isopycnal drifters are constrained to stay at a constant density, and obviously the water density following those particles does not change.

\subsection{Idealizing the plume front/copepod interac- tion}

In the introduction, we discussed how the model considered in Epstein and Beardsley (2001) may be an oversimplification of the system, most notably that plume fronts in the southwestern GOM are not infinitely wide, but were actually finite in their cross-shore extent. Until this point, however, we have not tried to quantify the differences between that model and the layer and ROMS models. In the following section, we address this point. First, we derive a scale for a simple front analogous to that considered in Epstein and Beardsley (2001) and compare it to what is observed in the layer and ROMS models. As we will see, there are discrepancies between them. We attempt to reconcile these differences by considering a model that bridges the gap between the more-realistic ROMS and layer models and the more idealized models, which helps us to simply understand the effect of a buoyant plume front on the distribution of copepods. 


\subsubsection{Scaling for expected concentration increase: one-dimensional "plow" model}

Lets begin at the simplest case, a one-dimensional front. In this model, we have an infinitely-wide front moving with some speed $c$ into a region of uniform initial copepod concentration. In this case, we ignore any along-front variability. As the front contacts copepods, the concentration at the front increases (analogous to a snow plow moving down the street, with snow piling up at the front of the plow). Diffusion acts to inhibit concentration growth, and some concentration is diffused behind the front. Figure 3-27 shows a concentration snapshot as a function of distance from the front.

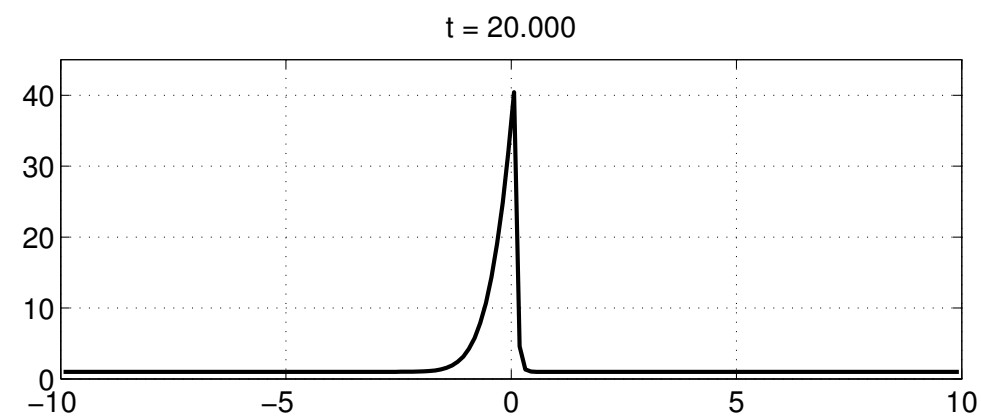

Figure 3-27: Snapshot of concentration as a function of distance from the front. The front is moving from left to right.

The concentration at the front is determined by the flux of copepods into the front (proportional to the propagation speed of the front) and the diffusion of copepods out of the frontal region:

$$
\frac{\partial b}{\partial t}=-\frac{\partial}{\partial x}\left(c b-\kappa \frac{\partial b}{\partial x}\right)
$$

where $b$ is the copepod concentration, $c$ is front speed, and $\kappa$ is the diffusivity.

The concentration at the front changes proportional to the flux in from in front of the plume (equal to $c b_{0}$, where $b_{0}$ is the ambient concentration), while the flux away from the front is due simply to diffusion (equal to $\frac{b \kappa}{L}$, where $L$ is the diffusive length of the plume, which is equal to $\sqrt{\kappa t}$ ). Thus the balance equation becomes: 


$$
\frac{\partial b}{\partial t}=-\frac{1}{\delta x}\left(c b_{0}-\sqrt{\frac{\kappa}{t}} b\right)
$$

This equation yields a scale for the expected concentration increase as a function of time:

$$
\frac{b}{b_{0}} \approx \sqrt{\frac{t c^{2}}{\kappa}}
$$

In this simple one-dimensional model, we expect the concentration to increase roughly as a function of $\sqrt{t}$. Plotted in figure 3-28 is the numerically-calculated concentration at the front as a function of time (blue line). Indeed, we see that the concentration does increase as $\sqrt{t}$. Again, this is what one might expect from an infinitely wide front.

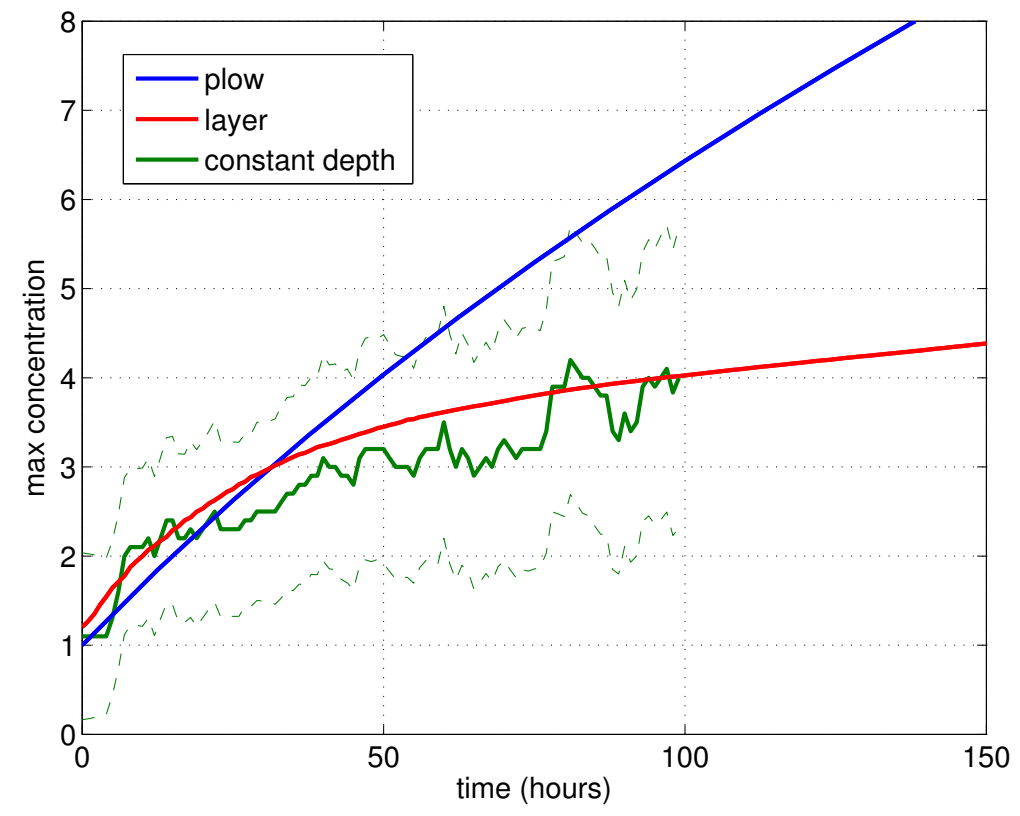

Figure 3-28: Maximum copepod concentration (copepods $/ \mathrm{m}^{3}$ ) in the simple "plow", average layer, and depth-keeping ROMS models. Dashed lines are 1 standard deviation above and below ROMS concentration.

Also plotted in figure 3-28, we see the maximum concentration from an average layer model run (red line) and maximum depth-keeping particle density from ROMS (green line). These two concentrations increase more slowly than the simple plow 
model, roughly like $\log (t)$ rather than $\sqrt{t}$. As we've seen, even the highly idealized plumes discussed in previous sections are not infinitely wide, and they also have circulation relative to the front that is not included here. What happens when we have this front-relative circulation? For this, we consider a two-dimensional front:

\subsubsection{Two-dimensional "plow": a curved front with front- relative circulation}

The question we now address is whether the front- (or plume-)relative circulation accounts for the slower growth in time of the concentration at the front. Consider now a two-dimensional "plow" model, where the front is curved as in figure 3-29.
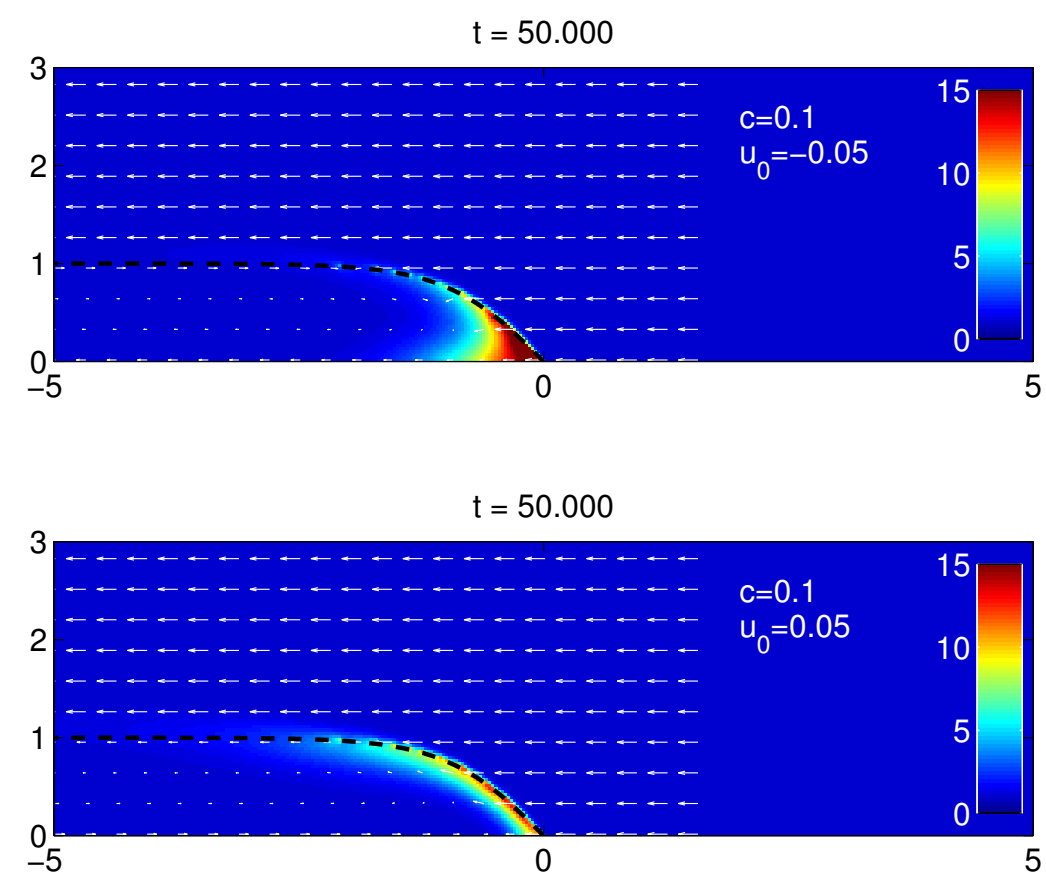

Figure 3-29: 2D plow examples. Colors are biological concentration, vectors are frontrelative flow, $u_{0}$ is flow along wall relative to front (positive is towards front, negative away), which is delineated by the black dashed line.

The front in this model is steadily advancing (from left to right in figure 3-29) at a speed $c$, and the concentration builds up at the front. In addition to diffusion away from the nose, there is advection by the plume-relative circulation within the 
plume, which tends to remove concentration from the nose and advect it away, either along the coastal wall (analogous to the layer model), or along the offshore edge of the front (analogous to the ROMS drifters). Depending upon the direction of this plume-relative flow, the concentration either builds up near the intersection of the front with the wall, or along the extension of the front offshore. Here, we define the plume-relative flow by the parameter $u_{0}$, which is the flow relative to the front along the coastal wall (positive is towards the front, negative is away, see figure 3-29). Figure 3-30 shows the maximum concentration in the model as a function of time for several values of the parameter $u_{0}$.

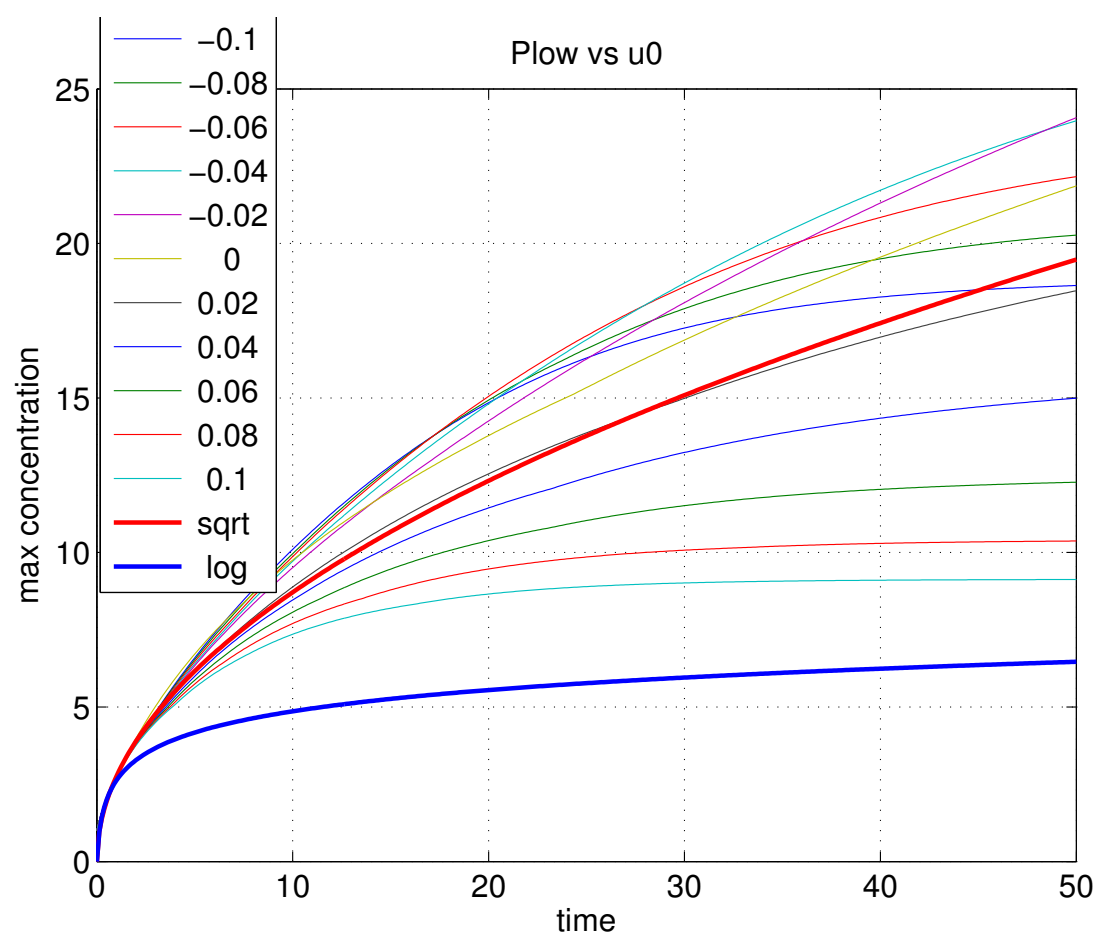

Figure 3-30: 2D plow concentration for different values of $u_{0}$. Here, $c=0.1$. Thick red and blue lines represent logarithmic and square root idealizations. Time units are hours.

By changing the sign and magnitude of $u_{0}$, we can alter the sense and strength of the front-relative circulation in order to understand its effects on the concentration at the front. Figure 3-31 shows the maximum concentration in the model as a function of time for many of the 2D plow experiments (gray), all the layer-model experiments 


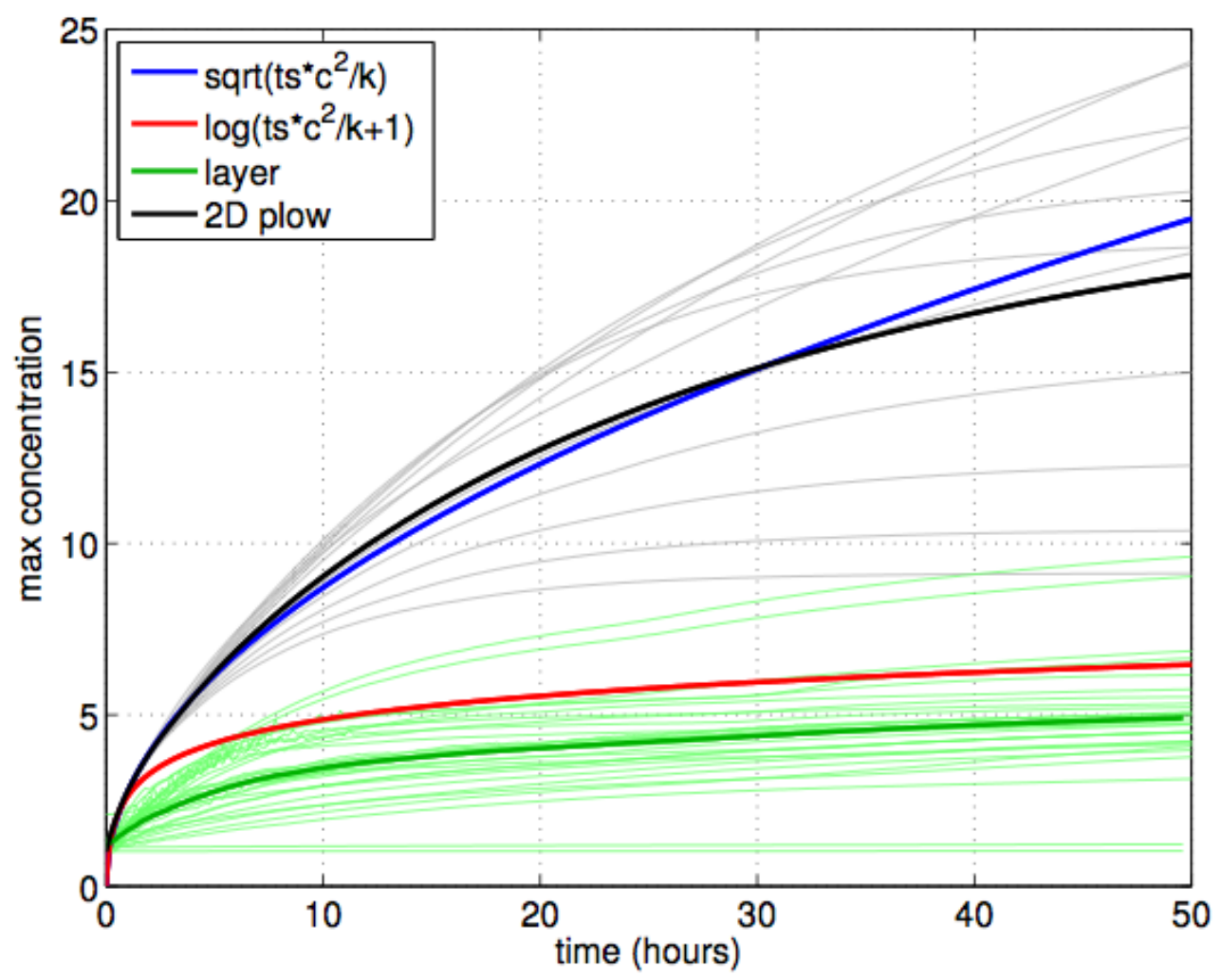

Figure 3-31: 2D plow vs. layer model. Red and blue lines represent logarithmic and square root idealizations. The layer model increases slower than any of the $2 \mathrm{D}$ plow models.

(green), as well as two theoretical models for the growth in time of the maximum concentration $(b \approx \sqrt{t}$, as shown from the $1 \mathrm{D}$ plow model, and $b \approx \log (t)$, as observed in the layer model and ROMS drifter concentration). Here, we vary $u_{0}$ from -.05 to .15 (where $c=0.1$ ). For reference, $c_{p}$ tends to be between 1-2x as large as $u_{0}$ for the different layer model runs, while for ROMS the nose speed is roughly $1.5 x$ faster than the plume-relative flow along the wall.

The maximum concentration that results is larger for $u_{0}<0$; this is because the concentration that builds up all along the front is advected towards the stagnation point where the front intersects the wall. When $u_{0}>0$, the circulation moves concentration along the front away from the wall, thus resulting in a smaller increase in concentration. From this we can see that concentration in the $2 \mathrm{D}$ plow model in- 
creases slower than $\sqrt{\frac{t c^{2}}{\kappa}}$, but not quite as $\log \left(\frac{t c^{2}}{\kappa}\right)$, as the layer model approximately does. The addition of plume-relative circulation explains some of the discrepancy between the 1D plow and the layer model results, but not all of it.

\subsubsection{Slowing "plow"}

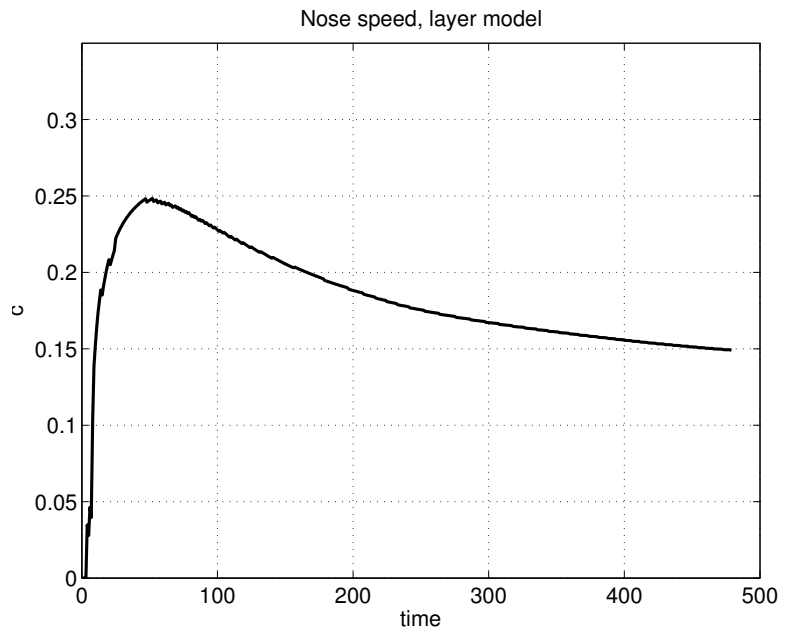

(a) Plume nose speed in layer model

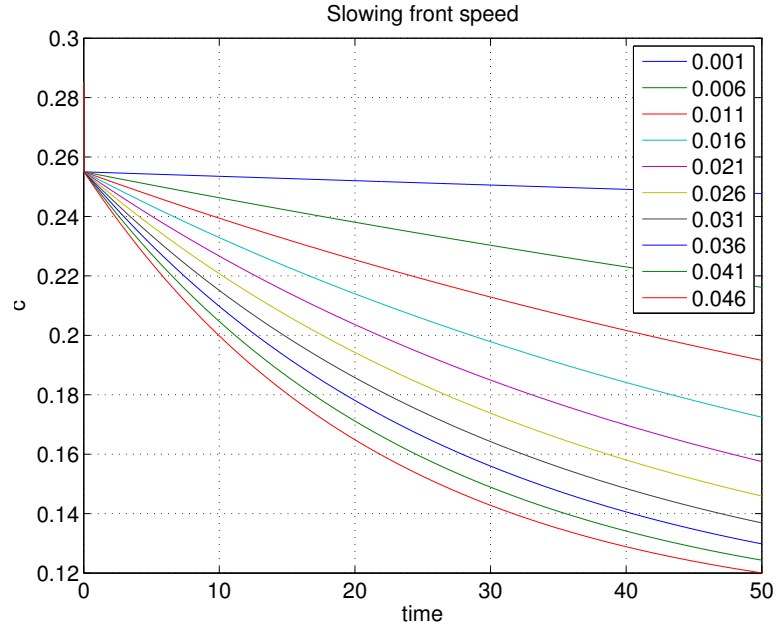

(b) Example front speed in plow model

Figure 3-32: Front speed $(\mathrm{m} / \mathrm{s})$ as a function of time. Front in plow model slows at a rate proportional to $\alpha$

So far in this section, we have only consider steadily-advancing fronts, however, plumes that form from steady freshwater sources actually slow down in time (e.g. Fong and Geyer (2002)). Indeed, the nose speed diagnosed from the layer model shows exactly this behavior (see figure 3-32). If the front slows down, then the flux of concentration to the front gets smaller in time, and may alter the resulting concentration at the front. Also of note, when the front slows down in the layer model, the plume-relative circulation also slows down such that the ratio between the two is roughly constant in time. How does this affect the resulting concentration increase in time?

In the "slowing plow" model, we consider the front speed to be a function of time 


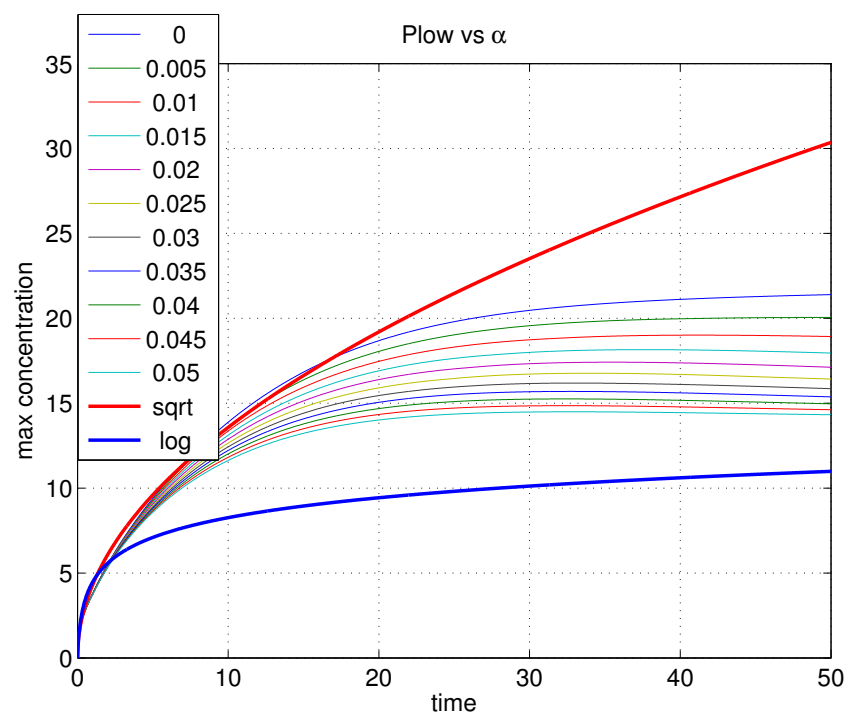

Figure 3-33: Maximum concentration as a function of time for 2D plow where front speed and plume-relative circulation slow by different fractions at each time step.

according to:

$$
c=c_{0}+c_{1} e^{-\alpha t}
$$

where $c_{0}=.105 \mathrm{~m} / \mathrm{s}$ and $c_{1}=.15 \mathrm{~m} / \mathrm{s}$, and $\alpha$ is the e-folding scale of the front speed. The e-folding scale of the plume front in the layer model is between .001 $\frac{1}{h r}$ and $.006 \frac{1}{h r}$ (corresponding to decay times scales roughly between 200 and 1000 hours). Figure 3-33 shows the maximum concentration that results from the slowing 2D plow model for several different $\alpha$. In the limit where the front doesn't slow much $(\alpha=0)$, we end up with the steadily-moving front results from the previous section. As $\alpha$ increases, however, we see that the concentration increase is inhibited, and for large-enough $\alpha$ the concentration actually decreases with time. For large $\alpha$ (or long-enough time), the front will slow to a point where diffusion and/or advection of concentration away from the nose is stronger than the increase of concentration due to movement of the front; at this time the concentration will begin to decrease. 


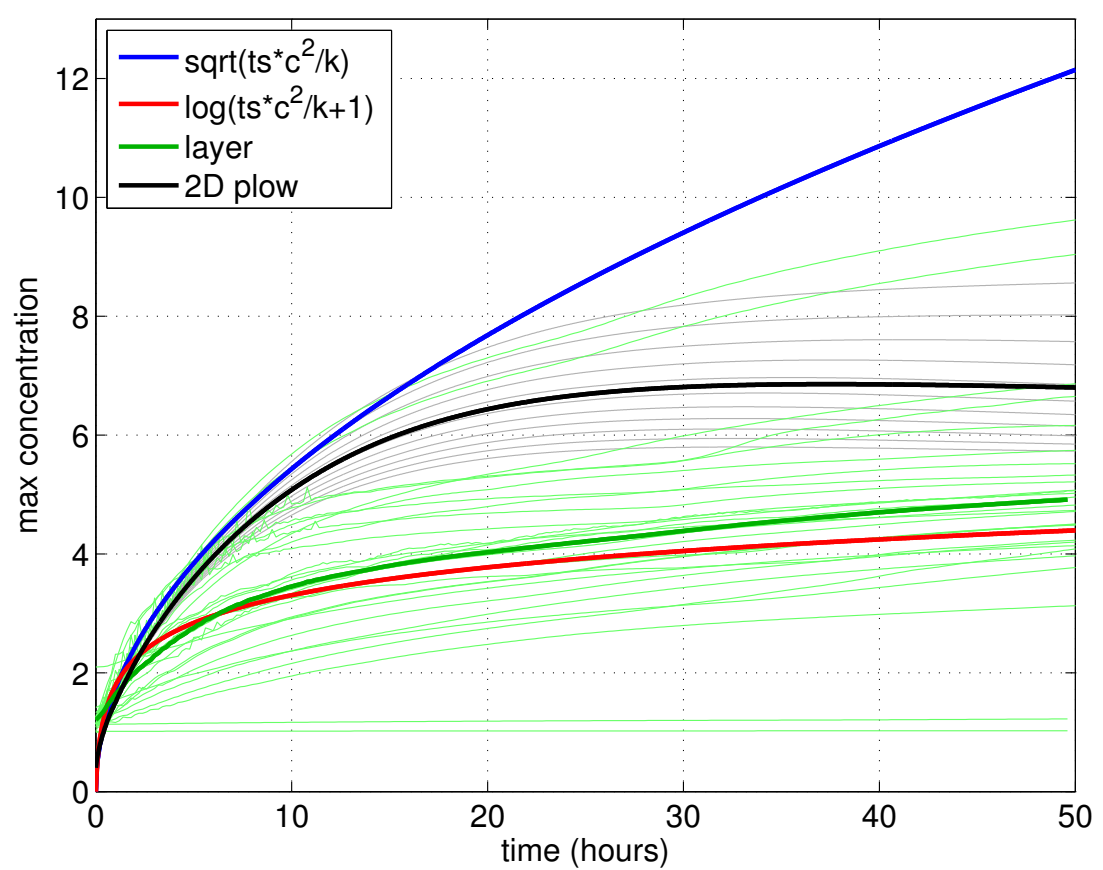

Figure 3-34: 2D plow vs. layer model. Red and blue lines represent logarithmic and square root idealizations. The layer model increases slower than any of the $2 \mathrm{D}$ plow models.

\subsection{Discussion/Conclusion}

The important contributions and inferences from this work are summarized as follows:

- Observations and realistic numerical models indicate that the southwestern Gulf of Maine contains salinity fronts, buoyant plumes, and associated lateral convergence that, when combined with depth-keeping copepod behavior, may result in aggregations of copepods qualitatively consistent with the hypothetical aggregation mechanism of Epstein and Beardsley (2001) (section 3.2). Recall that observations of copepod density in dense patches is roughly an order of magnitude or two larger than background concentrations (Epstein (1995), Baumgartner and Fratantoni (2008)). Model results show that amplification of copepod concentration ranges from 1 to 30 times the initial concentration (see, for example, figure 3-34), consistent with observations. 
- For depth-keeping organisms interacting with a buoyant plume, the resulting maximum concentration is shown to scale with $S \sim \sqrt{\frac{h_{p}}{d_{b i o}} \frac{W_{p} c_{p}}{\kappa}}$, while the area of the resulting patch scales with $S_{a} \sim \sqrt{\frac{d_{b i o}}{h_{p}} \frac{\kappa f}{c_{p}^{2}}}$ (section 3.4.3).

- Different copepod "behaviors"-namely, isopycnal vs. depth-keeping vs. passive drifting-result in very different copepod distributions when interacting with the same plume. Particles/copepods that are initially deeper than the plume are not affected by the plume as much, and are mostly similar to one another (section 3.4.4)

- Passive particle concentrations do not change (since particles are following incompressible flow), but can become horizontally or vertically closer to one another, on average.

- Depth-keeping particles deployed near the surface (such that they directly interact with the plume) are compressed laterally, and on average become much closer together

- Isopycnal particles do not compress laterally more than average passive particles, but are compressed to a high degree in the vertical, resulting in much higher numbers of particles within a certain distance, on average.

- A simple scale for the expected growth in concentration can be found for a simple one-dimensional "plow" model, where $b \sim \sqrt{t c^{2} / \kappa}$, which is faster than either the layer model or ROMS, which exhibit a behavior more like $b \sim \log t c^{2} / \kappa$. Including two-dimensionality and a non-steady front speed in the "plow" model compensates for the difference (3.5).

An important contribution of this work is that we are now able to bridge the gap conceptually between the idealized Epstein and Beardsley (2001) model and realistic, three-dimensional plumes and fronts in the southwestern GOM. Specifically, we're able to compenstate for the discrepancy between the theoretical concentration increasing as $\sqrt{\frac{t c^{2}}{\kappa}}$, while the layer and ROMS models show slower growth $\left(\log \frac{t c^{2}}{\kappa}\right)$. 
From the idealized work in section 3.5, we can now think of a buoyant plume and front as a curved plow that is slowing down as it gets farther from its source. Behind the front there is some relative circulation which changes the resulting distribution of copepods, and inhibits the growth in maximum concentration.

Epstein and Beardsley (2001) noted a salinity front near the patch of copepods that motivated their study, but there haven’t been many other observations of patches collocated with fronts, bringing the front mechanism into some question. The work above provides a few alternative hypotheses. First, the front-relative circulation may take enhanced copepod concentrations and move them away from the front. Thus, when these patches of enhanced concentration are observed, they may not be near the front that caused the concentration to increase in the first place. Second, if the copepods are not swimming to stay at a fixed depth but rather are sitting on a certain isopycnal surface, they may move under the plume and become compressed vertically more so than horizontally. This may enhance concentrations not near the front itself but some distance away from the front.

One copepod behavior not explicitly discussed in the modeling study but often observed in the wild is diel vertical migration (DVM, figure 3-3). While we haven't explicitly modeled the effect of this behavior, we can hypothesize about what to expect in this case. Assume that the copepods are swimming up as a uniform sheet towards the surface at night time ( such that $\left.\frac{\partial w_{s w i m} C}{\partial x}=0\right)$; when the copepods reach depths shallower than the plume, the concentrations will begin to rise at the nose and will continue to rise for as long as the copepods are at the surface. The big difference between this and the concentration of organisms sitting always at the surface is that the concentration for the DVM organisms is limited by how long they are at the surface. If, however, the ambient initial concentration is high enough, then the limited increase during the time when the copepods are at the surface may be sufficient for a dense aggregation to form. Also, note that the copepod concentration can increase through the swimming behavior alone, if $\frac{\partial w_{\text {swim }} C}{\partial x}<0$ (i.e., the copepods are swimming towards higher concentrations, thereby increasing the copepod concentration 
completely independent of the physics). 


\section{Chapter 4}

\section{Surface currents and eddy diffusivity estimates in the Gulf of Maine}

\subsection{Introduction}

The Gulf of Maine (GOM) is an ecologically and economically important region off the coast of New England. Many species of marine mammals and important fisheries species inhabit the waters of the GOM. The circulation in the GOM plays an important role in determining the distribution of the plankton, which in turn are a vital part of the marine ecosystem. In the previous two chapters we discussed a mechanism through which the GOM circulation could generate a dense aggregation of copepods at a salinity front associated with a freshwater plume. Now, we shift gears to discuss how the flow may take a patch and spread it out. This chapter focuses on the variability of the surface flow in the GOM in order to better understand its role in determining the distribution of copepods. Surface drifters have been used in many regions of the world's oceans to understand the circulation (e.g. Davis (1991), Swenson and Niiler (1996), Brink et al. (2003), Lacasce (2008)). In this work, a surface drifter dataset (Manning et al. (2009)) and numerical drifter simulations will be 
used to quantify the variability of the surface flow in the Gulf of Maine. The main questions addressed in this chapter are:

1. What are the patterns and structures in the mean GOM circulation?

2. What are the spatial and temporal patterns in eddy kinetic energy and eddy diffusivity?

Manning et al. (2009), Brink et al. (2003), and Naimie et al. (2001) discuss in some part the circulation of the Gulf of Maine as observed from surface drifters, and some of their data is used in this work. Brink et al. (2003) and Naimie et al. (2001) focus in particular on the Georges Bank circulation, while Manning et al. (2009) considers the GOM overall. What follows in this study is an updated picture of the circulation from these past studies. With more data and new techniques, we are able to obtain a higher spatial resolution and higher statistical significance than previous studies. Also, we attempt to characterize spatial and temporal variability in eddy diffusivity using both single-particle and pair statistics, which to our knowledge has not been attempted in past studies due to lack of sufficient data.

The main goal of this chapter is quantifying the variability of the flow. The second part of this study (in a subsequent chapter) evaluates the methods used in the Lagrangian analyses, but this chapter focuses on the results in particular. The main data used in this chapter is a 20-year archive of surface drifter data (Manning et al. (2009)), which is discussed in section 4.2. Section 4.3 explain methods used in this work to analyze the Lagrangian data. In section 4.4, the mean, eddy kinetic energy (EKE), and eddy diffusivity are computed from the drifter dataset. A discussion and conclusion section follows in section 4.5.

\section{Background}

In order to understand the role the flow plays in determining the distribution of tracer in the flow, one traditionally begins with the advective-diffusive equation. This 
equation can be written for a passive tracer $C$, following Lacasce (2008) and Davis (1991), as:

$$
\frac{\partial}{\partial t} C+U \cdot \nabla C=-\nabla \cdot<u^{\prime} C^{\prime}>=\nabla \cdot(\kappa \nabla C)
$$

where $U$ is the mean flow and $\kappa$ is the effective diffusivity. Understanding how the tracer is redistributed by the flow basically amounts to understanding these two quantities. Note that equation 4.1 dictates how the flow moves and distributes things, including surface drifters and copepods. A similar equation can be written for biological quantities that includes biological behavior (e.g. swimming, growth, social behavior), but in this work we focus solely on a passive tracer in an incompressible fluid.

The mean advection of copepods and copepod patches, quantified by the mean flow $U$, is important because it determines in an average sense the paths along which copepods will travel. Lagrangian drifters can be used to quantify the mean flow on an Eulerian grid (e.g. Manning et al. (2009) used a 1/2 grid for the GOM), or on an adaptive grid (e.g. Koszalka and Lacasce (2010)). Both will be done in this work.

The tendency of a copepod patch to remain as a patch depends directly upon the forces (eddies) in the fluid trying to tear it apart. The strength of eddies can be quantified by eddy kinetic energy, EKE, and/or $\kappa$, the eddy diffusivity. Using drifters it is possible to characterize variability of $\kappa$ in space and time (e.g. Davis (1985), Davis (1991), Zhurbas and Oh (2003), and Lacasce (2008)).

There are many ways to quantify the mean flow and eddy diffusivity; we choose to approach the problem from a Lagrangian point of view, and utilize surface drifters. Copepods (and really anything that drifts with the flow, including plankton, pollutants, flotsam, etc.) are largely at the whim of the horizontal ocean currents, and thus may act similarly to a drifter. 

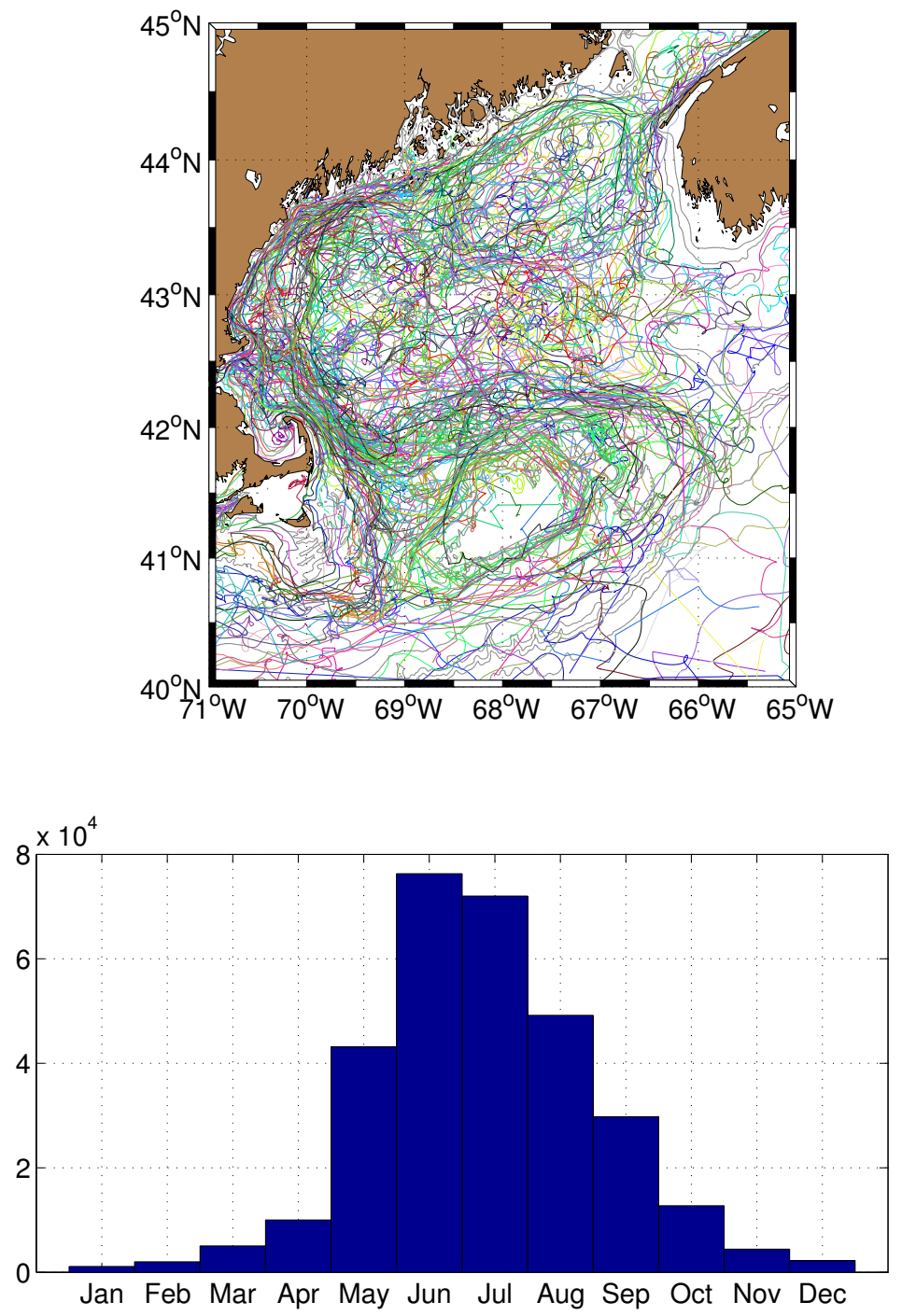

Figure 4-1: (left) All 363 drifter tracks used in this analysis. Each color is a separate drifter trajectory. (right) Distribution of drifter observations by month. Wintertime data coverage is too low to allow for seasonal analysis, so only summer-time is discussed herein.

\subsection{The drifter dataset}

\subsubsection{GOM Drifter Observations}

This study utilized surface drifter data collected by the NOAA's Northeast Fisheries Science Center as part of an ongoing mission to monitor and assess the Northeast 
Continental Shelf ecosystem. The observational data consists of 363 drifters deployed in the GOM from 1988-2010. Most (340) were drogued in the upper 15m, while 11 and 12 drifters were drogued at $40 \mathrm{~m}$ and $50 \mathrm{~m}$, respectively. The drifters were of the type described in Manning et al. (2009), and the raw data may be obtained from the Northeast Fisheries Science Center website (nefsc.noaa.gov). Figure 4-1 shows the tracks of all drifters, and the distribution of drifter observations by month. These observations are clearly heavily biased towards summer. Thus, the subsequent analyses only focuses on summertime conditions (May through September); data outside these times are discarded.

The hourly drifter positions were processed in the following way, analogous to commonly used practices with observations. Each drifter position time series is smoothed with the PL66 filter (Beardsley et al. (1985)), with a half-power of 38 hours, to remove tides and high frequency fluctuations. Low-pass filtered drifter velocities were then computed by first-differencing the drifter position. Drifter velocities were also computed from spline fitting, but the velocities obtained were essentially equivalent to first differences, so the former were used. Every drifter then has an associated time series of position and velocity.

\subsection{Methods}

\subsubsection{Computing summertime mean fields}

Since the temporal data coverage is so seasonally biased (figure 4-1), we only take the summertime data in all of the following analysis (May 1 through September 1). Two methods are employed to compute the mean velocity field and eddy kinetic energy (EKE) from surface drifters. The first is a traditional binning method, which has been undertaken in previous studies (e.g. Brink et al. (2003), Manning et al. (2009)). The data are grouped together in predefined geographic bins, and averaged together to obtain the mean velocity field. In this particular implementation, we use a smaller bin size $\left(0.1^{\circ} \times 0.1^{\circ}\right)$ than those previously used. To compute the mean velocity field, we 
take all velocity measurements within a bin and average them together. Bins through which lots of drifters pass naturally contain a more accurate (in a statistical sense) estimate of the mean flow. The Lagrangian decorrelation time scale in the GOM is roughly 1.5-3 days (Brink et al. (2003)). Thus, we only retain those bins in which we have more than 10 drifter days of data, corresponding to at least 3-7 independent estimates of the mean flow there. In addition to the mean flow, we compute EKE, defined as

$$
\mathrm{EKE}=\frac{1}{2}\left(<u^{\prime 2}>+<v^{\prime 2}>\right)
$$

where $u^{\prime}, v^{\prime}$ are the departures from the mean velocity field. $u^{\prime}, v^{\prime}$ are computed for each of the $0.1^{\circ} \times 0.1^{\circ}$ bins by removing the mean velocity field from each observation and averaging within the bin.

The second method to compute the mean velocity field is adaptive binning, or "clustering" (Koszalka and Lacasce (2010)). In this method, velocity observations are grouped together in bins shaped such that approximately the same number of observations are contained in each bin. The resolution of the grid is not regular; there will be smaller bins where there are more dense observations, and larger bins where there are no data. Here, we specify the number of clusters we wish to obtain by choosing the target number of observations per cluster. Then, following the method outlined in the appendix of Koszalka and Lacasce (2010), we use a software package (the MATLAB k-means toolbox) to obtain the locations of the cluster centers, and identify which observations belong to which cluster. The average velocity for a particular cluster is then computed in the same manner as the standard binning procedure.

\subsubsection{Computing diffusivity from single-particle statistics}

In equation 4.1, the eddy flux term has been parameterized as a diffusion with coefficient $\kappa$. This assumes that there is a clearly definable slowly-varying mean velocity field that has time and space scales of variation much longer than the fluctuations. In 
the GOM, we know the circulation varies seasonally (Brink et al. (2003)), but for the purposes of this study we assume that the circulation during the period May-August is quasi-stationary.

From Lagrangian particles, one can find the diffusivity $\kappa(\mathbf{x}, t)$ from (e.g. Davis (1991)).

$$
\kappa_{j k}(\mathbf{x}, t)=<u_{j}^{\prime}\left(\mathbf{x}, t_{0}\right)\left[\mathbf{x}_{k}-\mathbf{r}_{k}\left(t_{0}-t \mid \mathbf{x}, t_{0}\right)\right]>
$$

where the notation $r_{k}\left(t \mid \mathbf{x}, t_{0}\right)$ indicates the displacement at time $t$ of a particle at position $\mathbf{x}$ at time $t_{0}$, and $<>$ indicates an ensemble average of particles released at different $t_{0}$

As noted by Davis $(1991), v_{j}^{\prime}\left(t_{0} \mid \mathbf{x}, t_{0}\right)=u_{j}^{\prime}\left(\mathbf{x}, t_{0}\right)$, which gives

$$
\kappa_{j k}(\mathbf{x}, t)=-<v_{j}^{\prime}\left(t_{0} \mid \mathbf{x}, t_{0}\right) d_{k}^{\prime}\left(t_{0}-t \mid \mathbf{x}, t_{0}\right)>
$$

$u^{\prime}$ and $v^{\prime}$ are the departures from the Eulerian and Lagrangian velocities, respectively, and $d^{\prime}$ is the departure of the Lagrangian displacement from the mean Lagrangian displacement $D$ (calculated as the displacement due to the mean velocity). Equation 4.3 is the typical way that diffusivity is computed from Lagrangian trajectories. It is important to note that $d^{\prime}$ is computed backward in time.

In this derivation, $u^{\prime}\left(t \mid \mathbf{x}, t_{0}\right)$ is defined as the departure from the mean Eulerian velocity following a particle, which is different than the departure from the mean Lagrangian velocity $v^{\prime}$. For mean flows that vary only on large spatial scales, (large when compared to the trajectory segment used to compute $\kappa$ ), the two are roughly equivalent. In the Gulf of Maine, however, the velocity field varies on smaller scales (on the order of kilometers). For this calculation in the Gulf of Maine, is it more appropriate to remove the Lagrangian or Eulerian mean? Both will be used in this work to determine which of the two is appropriate.

Practically, we compute diffusivity from equation 4.3 following the method outlined by Swenson and Niiler (1996). Specifically, $v_{j}\left(t \mid \mathbf{x}, t_{0}\right)$ is the velocity at time $t$ of a particle at a location $\mathbf{x}$ at time $t_{0}$. As in Swenson and Niiler (1996), we treat every 
observation point in an area of question as the start of its own pseudo-trajectory, thereby improving the statistics, especially for short lags. For every trajectory, we must compute two terms:

- $u_{j}\left(t_{0} \mid \mathbf{x}, t_{0}\right)$ is the velocity at time $t_{0}$ of a particle at a location $\mathbf{x}$ at time $t_{0}$.

- $d_{k}\left(-t \mid \mathbf{x}, t_{0}\right)$ is the time series of the displacement of a particle ending at location $\mathbf{x}$ and time $t_{0}$.

We then take the product of the displacement $d^{\prime}$ with the associated velocity $u^{\prime}$, and take the mean to get $\kappa_{j k}(t) . \kappa_{j k}(t)$ has four components which are presented in the following manner: The diagonal components $\kappa_{x x}$ and $\kappa_{y y}$ are retained as is, and represent the stretching in the zonal and meridional directions, respectively. The anti-symmetric part of the tensor $\frac{1}{2}\left(\kappa_{y x}-\kappa_{x y}\right)$ represents the tendency of a parcel to rotate in a cyclonic fashion.

\subsubsection{Two-particle dispersion and diffusivity}

An additional method to compute dispersion from drifters is to look at the rate of separation of a pair drifters (Lacasce (2008)). We can define the relative dispersion $<\Delta(x, y)^{2}>$ as

$$
<\Delta x^{2}>=\sum_{i, j}\left(x_{i}-x_{j}\right)^{2} \quad<\Delta y^{2}>=\sum_{i, j}\left(y_{i}-y_{j}\right)^{2}
$$

where $i, j$ are the indices of individual drifters deployed in the same location at the same time. From this, we can compute the relative diffusion by taking a time derivative:

$$
\kappa_{\text {pair }(x, y)}=\frac{1}{2} \frac{d}{d t}<\Delta(x, y)^{2}>
$$

Equations 4.3 and 4.5 are both a measure of the diffusivity, but represent slightly different properties of the flow. Equation 4.3 is calculated from single drifter trajectories, and then averaged to provide a statistical picture of how the motion of a particle 
is correlated with itself. In contrast, the pair diffusivity in equation 4.5 provides information about how two particles are correlated with one another. In a statistical sense, both provide a measure of the spreading of a tracer due to fluctuations in the flow, and ostensibly should be equivalent. As we will see, however, this is not necessarily the case, largely because of the inclusion of spatially-correlated motion (e.g. wind-driven flow) that appear in the single-particle diffusivity as eddy activity but not in the pair statistics.

\subsection{Results-Observations}

\subsubsection{Mean flow and EKE, $0.1^{\circ} \times 0.1^{\circ}$ grid}

The mean velocity field and EKE for all drifter observations in the Gulf of Maine is shown on a standard $0.1^{\circ} \times 0.1^{\circ}$ grid in figure 4-2. The observed pattern of the surface velocity field, consistent with previous studies, is as follows: the general tendency of the circulation in the GOM is cyclonic, and there is a coastal current flowing around the perimeter of the GOM in the direction of Kelvin wave propagation. This coastal current turns in the Great South Channel and follows the northern edge of Georges Bank. There are two main exit pathways for surface fluid that are visible in this picture. The first is the Great South Channel, where flow moving southward along Cape Cod exits the GOM. The second is eastward flow along the northeast peak of Georges Bank, which exits the GOM through the southern part of the Northeast Channel. Finally, there is a meandering jet that circumvents the coastal current, splitting off near Mount Desert Island, ME, and short-circuiting to the northern flank of Georges Bank.

The patterns in EKE are subtle, but perhaps not surprising. The EKE is highest in the vicinity of strong flow, including the coastal current and Georges Bank, while lower in the interior of the GOM. This suggests that there are lots of energetic events around the edges of the Gulf of Maine, while there are relatively fewer in the interior. We may expect then to find higher values of diffusivity on the edges of the coast; this 

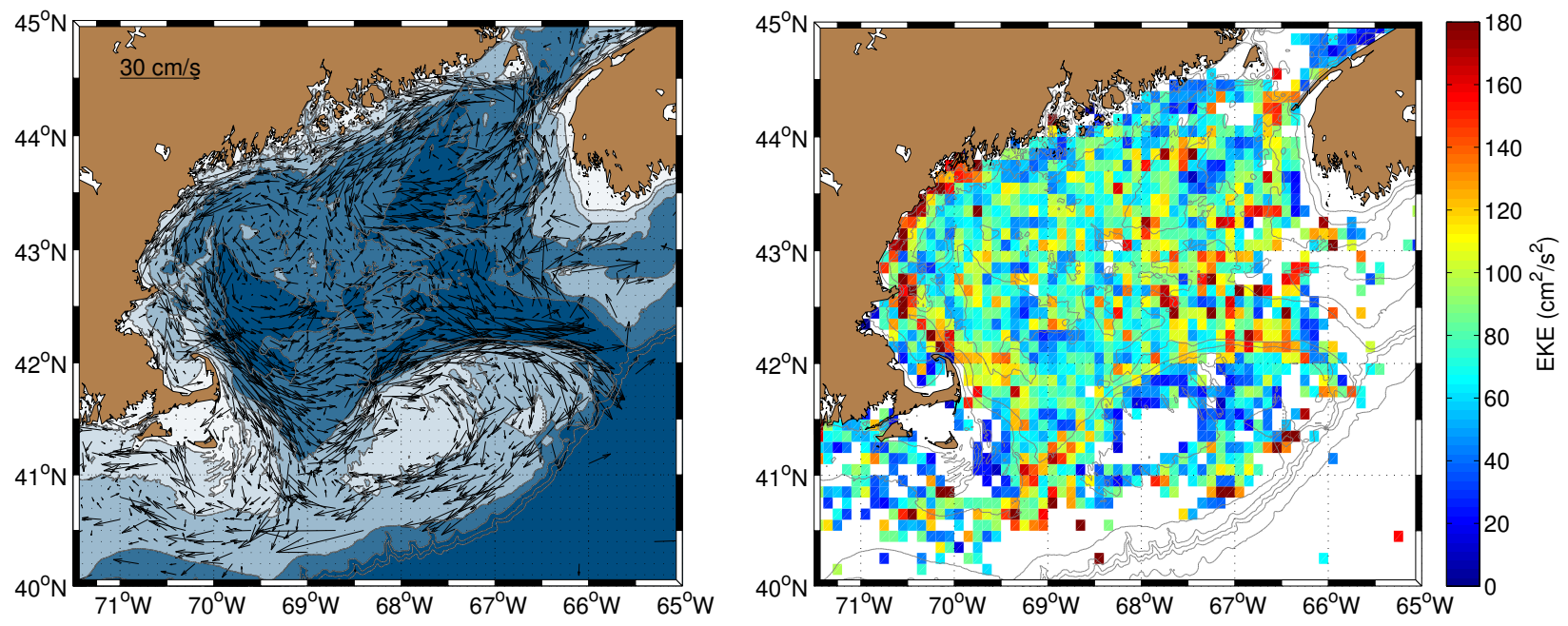

Figure 4-2: Mean velocity field (left) and EKE (right) from surface drifters, $0.1^{\circ} \times 0.1^{\circ}$ bins. Bins with fewer than 5 drifter days have been removed.

will be investigated further in a subsequent section.

\section{Clustering method}

The mean flow and EKE computed via the clustering method is shown in figure 4-3.

Here, we have chosen to have 920 clusters, which corresponds to roughly 300 individual observations per cluster. The resolution of the clustered product is roughly equivalent to that of the standard grid along the coastline where data coverage is large, but is not as good in the interior GOM and along the shelf south of the GOM. Qualitatively, the patterns in the flow are similar between the two pictures: again the presence of the coastal current, northern flank flow, and relatively quiescent interior are obvious. One notable difference between the two pictures is the flow in the northern part of the Great South Channel. Following the pathway of the coastal current from Cape Cod through the northern GSC to where it connects with the northern flank of Georges Bank, it is difficult to discern any change in the flow velocity or EKE in the gridded fields. In the clusters, however, one gets the sense that the flow slows down in the northern GSC between Cape Cod and Georges Bank, 

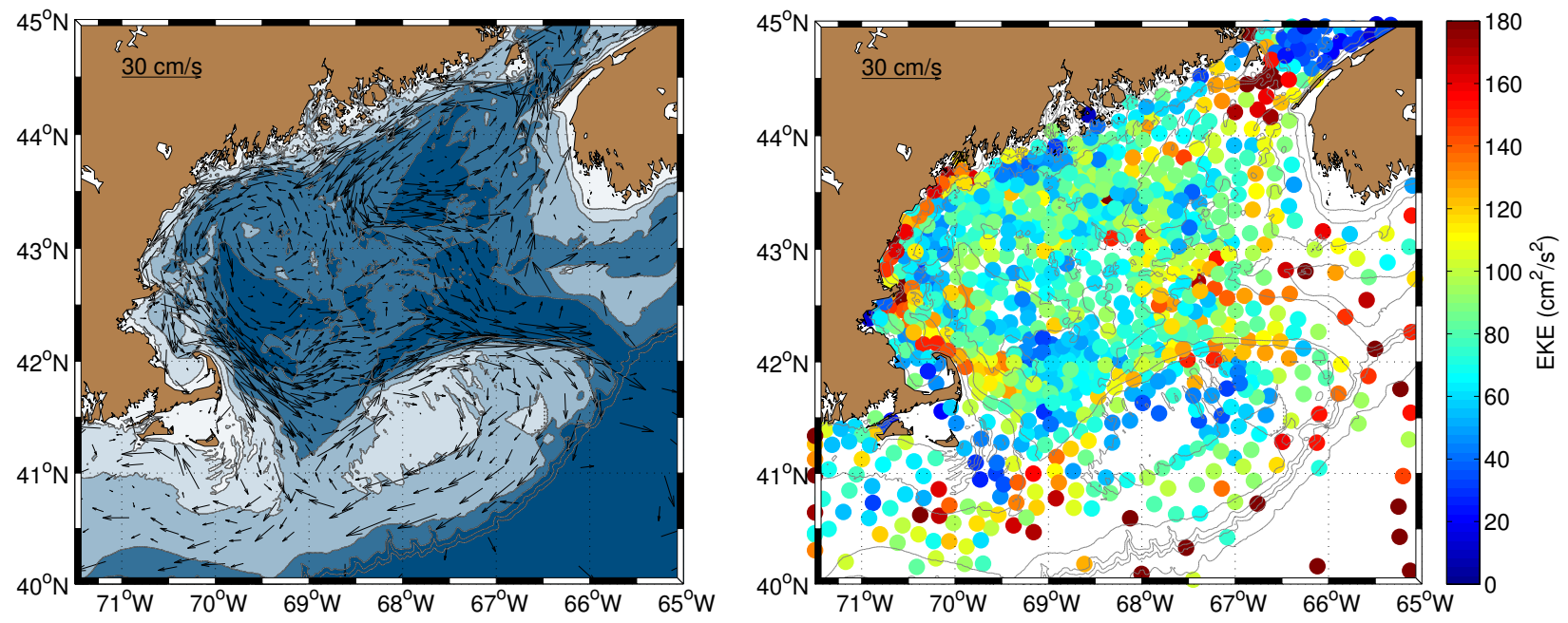

Figure 4-3: Mean velocity field (left) and EKE (right) from surface drifters in adaptive clusters. Vector scale is the same as in figure 4-2

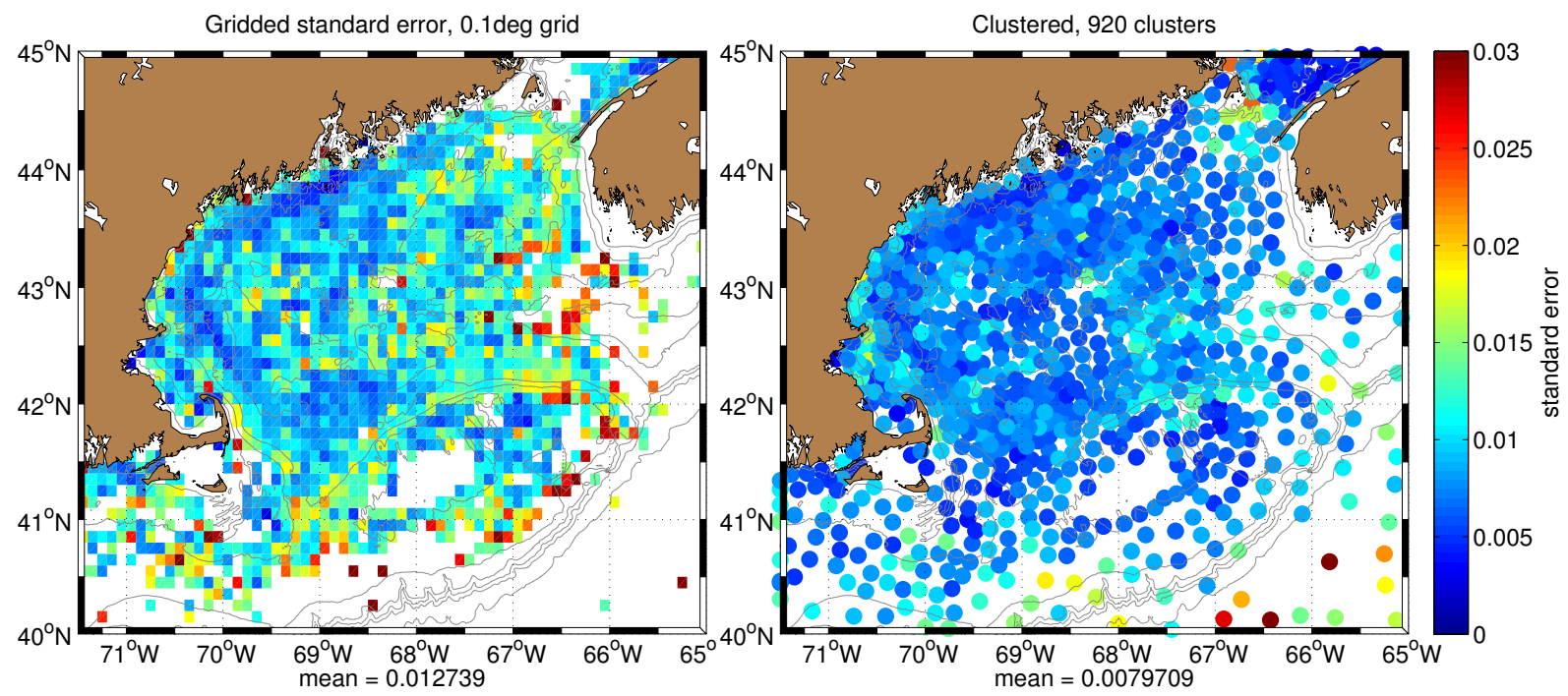

Figure 4-4: Standard error for gridded (left) and clustered (right) mean velocity estimates.

and that the EKE is actually lower there than on either side. This may be important for why whales tend to return to this region to feed as opposed to others in the GOM.

Perhaps more interesting are the standard errors associated with the averaging. Standard error is defined here as S.E. $=\frac{\sigma}{\sqrt{N}}$, where $\sigma$ is the standard deviation 
of members in the bin and $N$ is the total number of observations in the bin. In the gridded fields, the standard errors are naturally lower in bins with lots of observations (namely the bins close to shore, in the vicinity of the coastal current). The goal of the clustering approach is to standardize the number of observations in each bin. This would mean the standard error in each bin would depend solely on the standard deviation of the observations. Averaged over the entire domain, the standard error in the gridded domain is almost a factor of two larger than that of the clusters because of the large differences in number of observations per grid in the gridded domain.

\section{An updated view of the GOM mean surface flow}

We now compare the results of figures 4-2 and 4-3 with previous estimates of the GOM surface flow, and determine if there are any differences from the classical view of the circulation here. Figure 4-5 depicts a schematic of the surface flow in the GOM (adopted from He et al. (2005)). Also in figure 4-6 is the surface circulation from Manning et al. (2009), calculated on $\frac{1}{2}^{\circ}$ grid. It should be noted that Manning et al. (2009) did not remove the few drifters deployed during the winter months, which we removed to focus on the summer circulation. However, the seasonal bias in the full dataset is so drastic that Manning et al. (2009) reflects the summertime mean circulation similar to that presented above, and so we proceed assuming the bias is negligible.

The circulation in figure 4-5 is schematic, and so a quantitative comparison to our work is not feasible, however there is a discrepancy between the two which must be noted. Figure 4-5 indicates the presence of the SCOPEX gyre, a recirculating flow in the southwestern GOM where the coastal current along Cape Cod enters the northern GSC, turns eastward then northeastward along the northern flank of Georges Bank, then turns back to the west towards Cape Cod. This flow may be important in that it could retain copepods and copepod patches in the southwestern GOM for extended periods of time. Figure 4-3, however, does not show this presence of the SCOPEX gyre. The gyre could be present in the flow beneath the surface, but this flow does 


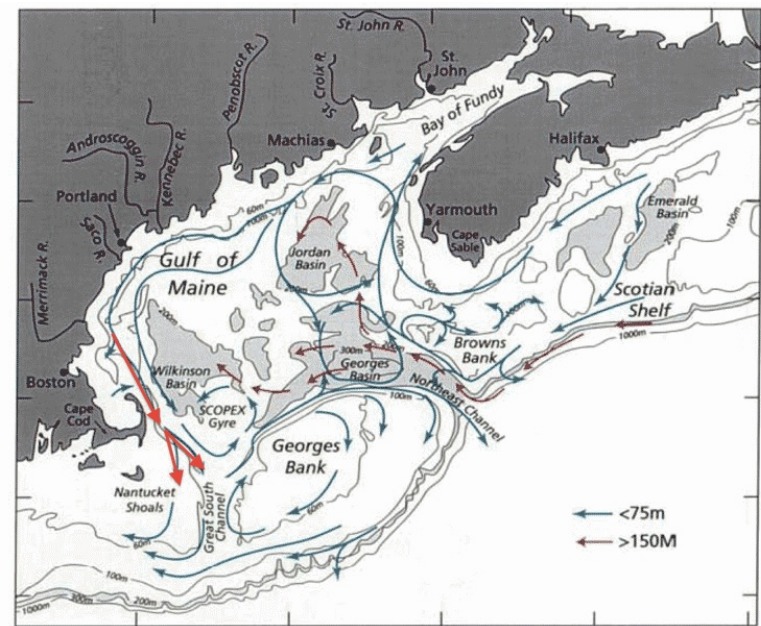

Figure 4-5: Surface circulation in the GOM (adopted from He et al. (2005))

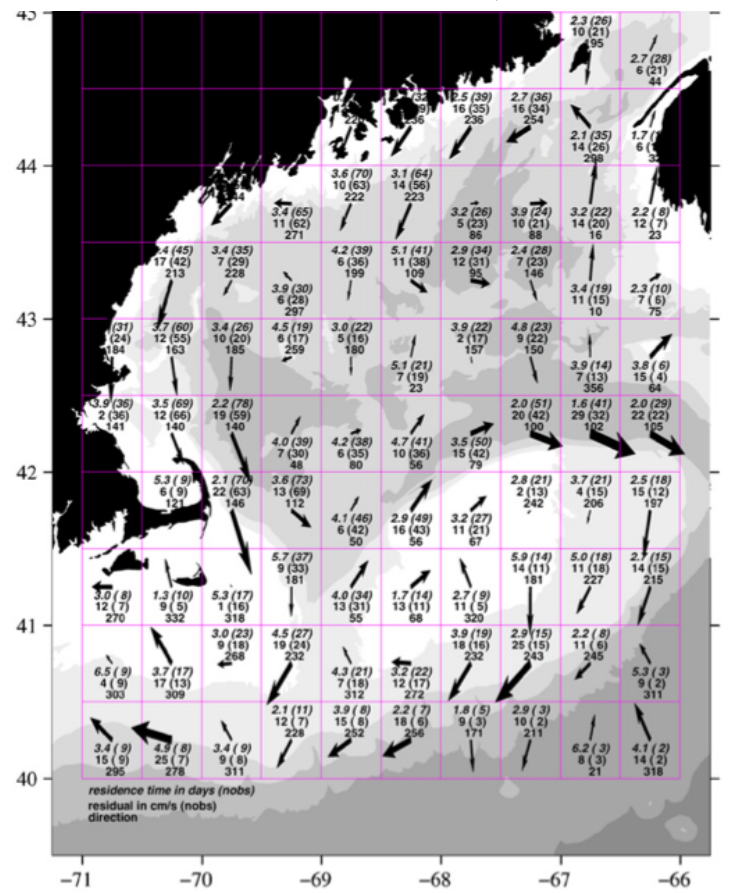

Figure 4-6: Mean flow from surface drifters on $\frac{1}{2}^{\circ}$ grid (from Manning et al. (2009))

not exist in this surface dataset.

The first difference one notices between the figure from Manning et al. (2009) and that of figure 4-3 is that the spatial resolution is much better, which makes morequantitative statements about the flow possible. For example, off the coast of Cape Cod, the width of the coastal current can be diagnosed (approximately $50 \mathrm{~km}$, less than the width of one $\frac{1}{2}^{\circ}$ grid cell, see figure 4-7). The fact that the grid used by 
Manning et al. (2009) is as large or larger than the width of the coastal current there means that the estimate of the flow speed is slightly reduced compared to the actual flow speed in the middle of the jet (e.g. $22 \mathrm{~cm} / \mathrm{s}$ for grid cell centered at $-69.75,41.75$ as opposed to $25 \mathrm{~cm} / \mathrm{s}$ for cluster nearest to that location in figure $4-3$, which is just to the south of the section shown in figure 4-7). Indeed, the reduction is small, and in fact is smaller than the standard deviation of the flow speed here. Similar effects are noticeable in other locations, including the strong flow along the northern flank of Georges Bank. Additionally, the lower resolution in Manning et al. (2009) almost completely masks the presence of the meandering jet that cuts between Wilkinson and Jordan basin (evident in the schematic in figure 4-5 and 4-3). Finally, the bifurcation of the coastal current at Cape Ann, with part of the flow turning in to Massachusetts Bay while part skirts along the eastern edge of Stellwagan Bank is noticeable in figure 4-3 but not in that of Manning et al. (2009).

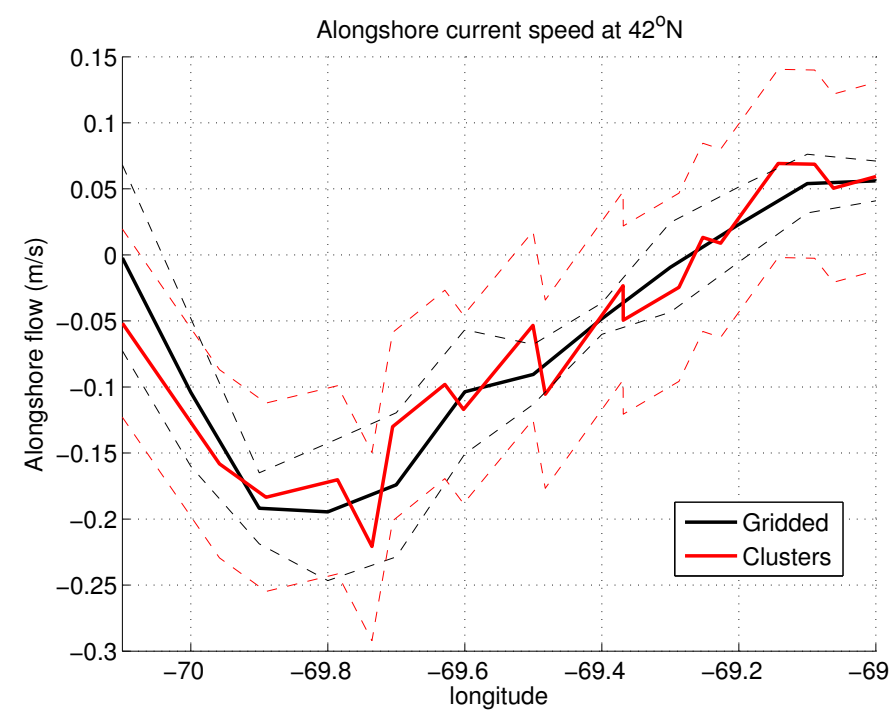

Figure 4-7: Alongshore flow section along $42^{\circ} \mathrm{N}$ extending from Cape Cod eastward into the GSC. Red (black) lines indicate speed from clusters (standard grid). Standard deviations are shown in the dashed lines. 


\subsection{2 $\kappa$ over the entire GOM}

To compute a single time-series of diffusivity from all available data, we first compute the mean Lagrangian displacement as a function of lag, shown in figure 4-8. The

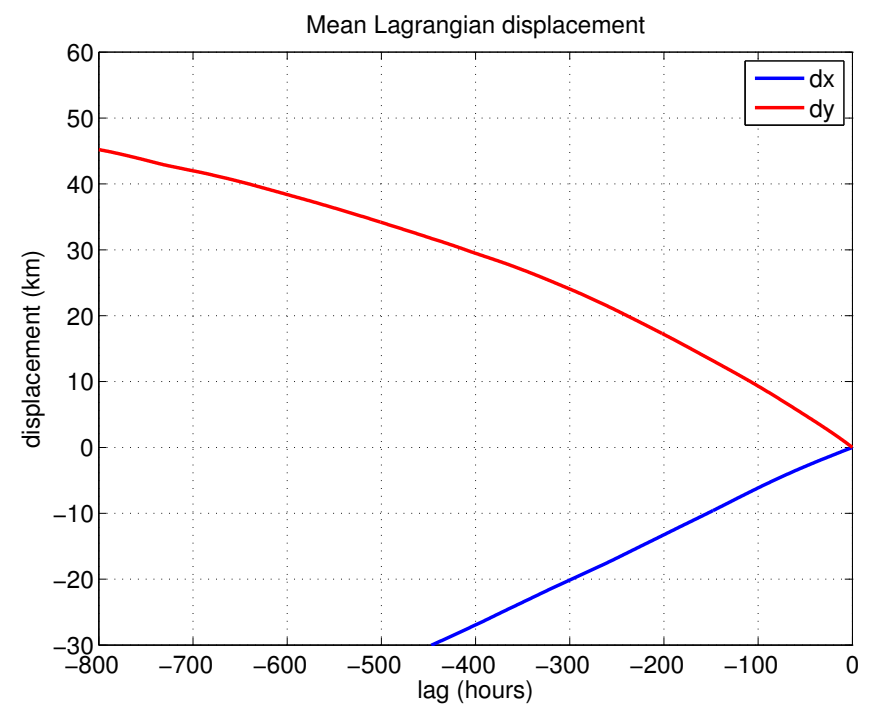

Figure 4-8: Mean Lagrangian displacements, real drifters

meridional displacement is increasingly positive for larger negative lags, indicating the tendency of southward flow in the Gulf of Maine. The zonal displacement is increasingly negative for longer negative lags, which reflects the tendency of the drifters to move from west to east, in particular along the northern flank of Georges Bank.

We can next compute the departures from the mean Lagrangian displacements and velocities and compute the diffusivity tensor, as was done in Brink et al. (2003). A rough estimate of the Lagrangian decorrelation time scale for the GOM is between 1.5 and 3 days, which indicates that the diffusivity should asymptote by about an order of magnitude longer than the decorrelation time, or by 20 days (Swenson and Niiler (1996)). For this reason, we show the diffusivities at lags up to 30 days (figure 4-9). For comparison, the identical computation from Brink et al. (2003) is shown also. In this figure, we show the diffusivity computed with Lagrangian mean removed (solid lines, $\kappa^{\text {traj}}$ ) and Eulerian mean removed (dashed lines, $\kappa^{\mathrm{Eul}}$ ). There is clearly a good comparison between $\kappa^{\text {traj }}$ and that in the companion figure from Brink et al. (2003). 

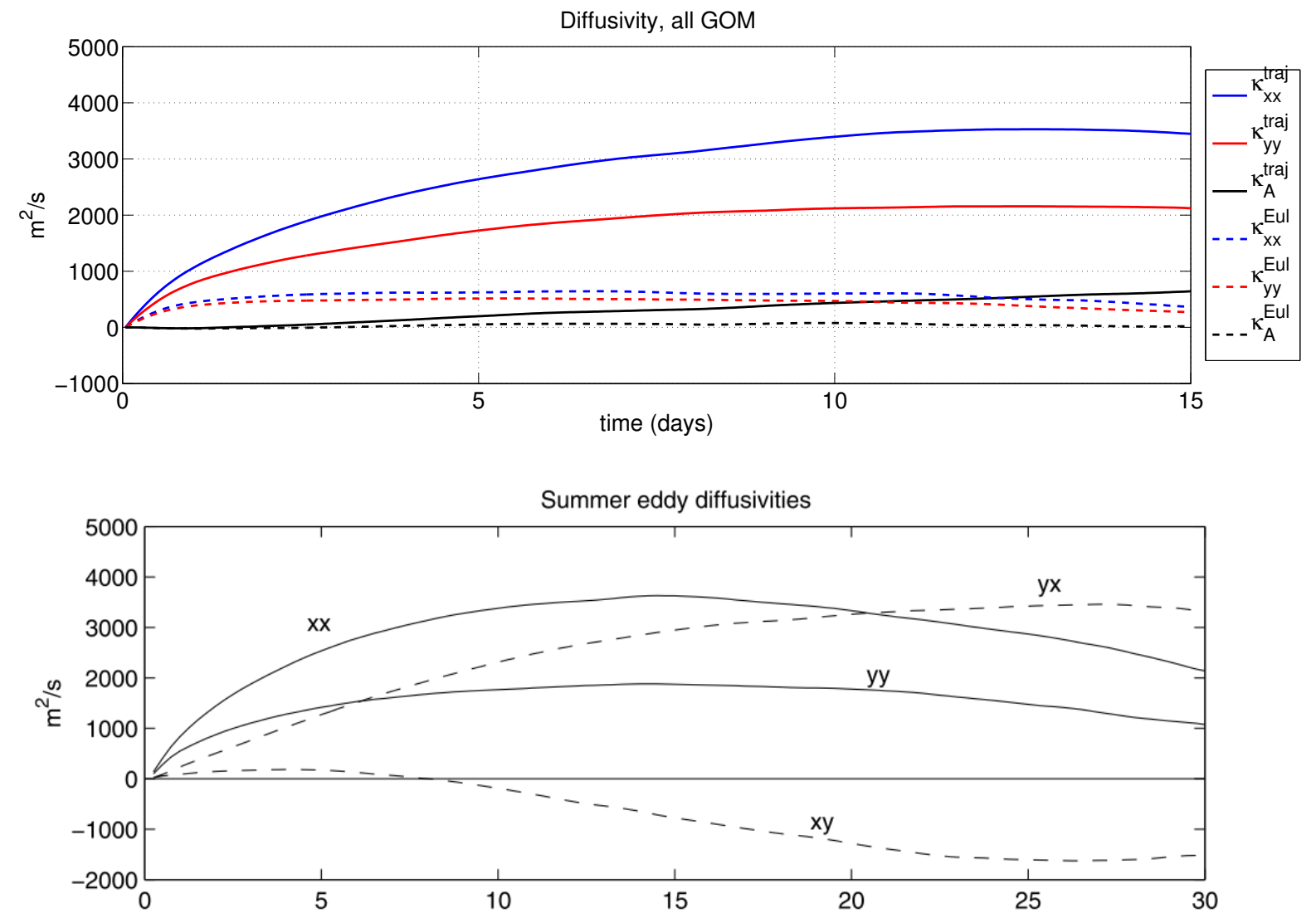

Figure 4-9: Eddy diffusivity tensor $\kappa$ for all drifters in the GOM (top), and from summer drifters as in Brink et al. (2003) (bottom). Time in both plots measured in days.

In both datasets, $\kappa_{x x}$ is almost twice as large as $\kappa_{y y}$. Single-particle diffusivities tend to be exaggerated in the direction aligned with a mean flow. There are more eastwest aligned flows in the GOM than north-south (see figure 4-2), particularly along the northern and southern flanks of Georges Bank. These east-west flows tend to accentuate the component of $\kappa$ in that direction.

Brink et al. (2003) also removed the Lagrangian mean velocity from the trajectories before computing $\kappa$. The patterns in $\kappa$ are similar between the two methods, but the values for $\kappa^{\text {Eul }}$ are much smaller than $\kappa^{\text {traj. }}$. While $\kappa^{\text {traj. }}$ is consistent with Brink et al. (2003), the more appropriate measure of eddy diffusivity is $\kappa^{\mathrm{Eul}}$. Consider the trajectory in red in figure 4-10. The spatially-variable Eulerian mean flow is shown in black, and the mean velocity associated with the trajectory (the Lagrangian mean) 
is in blue. In this example, some of the mean flow would remain after removing the Lagrangian mean from the trajectory, which would give a spuriously large eddy diffusivity. It is more appropriate to remove the spatially-variable mean flow from the trajectory, which would only leave the eddy velocity. Looking back to figure $4-3$, it is clear that the mean flow in the GOM contains lots of spatial variability, which may explain why $\kappa^{\text {traj }}$ is much larger than $\kappa^{\text {Eul }}$.

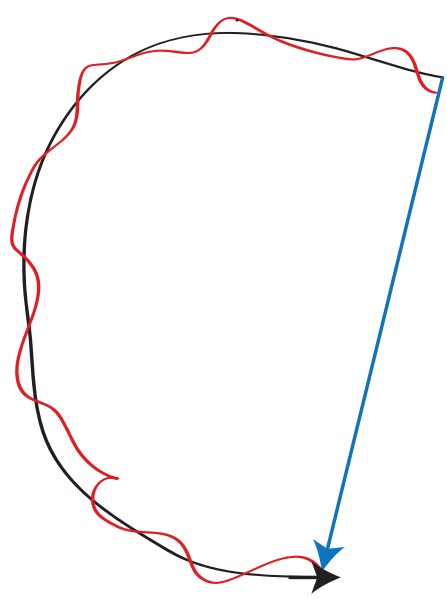

Figure 4-10: Example of Eulerian and Lagrangian mean for an imaginary drifter in the GOM. The trajectory of the drifter (red) closely follows the Eulerian mean flow (black), with small deviations due to eddies. Removing the Lagrangian mean flow calculated from this trajectory (blue) ignores the spatial variations in the mean flow, and would thus consider them to be part of the eddy field, leading to an artificially large estimate of the diffusivity.

Most studies ignore the off-diagonal components of the $\kappa$ tensor, but important information is contained therein. The anti-symmetric part of the tensor $\kappa_{A}=\frac{1}{2}\left(\kappa_{y x}-\right.$ $\left.\kappa_{x y}\right)$ indicates the tendency for particles to veer to the left (in a cyclonic sense). $\kappa_{A}$ is positive over the majority of the GOM, consistent with the cyclonic tendency of the circulation that is present year-round throughout the broader GOM.

\subsubsection{Pair dispersion}

In addition to the single-particle diffusivity, it is possible from pairs of drifters to compute a pair diffusivity, $\kappa^{\text {pair }}$. All drifter pairs used in this analysis are "chance 
pairs", meaning they were not necessarily deployed together, but rather just happen to pass within $2 \mathrm{~km}$ and 1 hour of each other during their lifetimes. Koszalka and Lacasce (2009) showed that the statistics from "chance pairs" were identical to that from pairs deployed together.

Figure 4-11 shows the number of pairs as a function of time since "release". Here, the "release" time is taken to be the time when the drifters are closest together. Also shown are the locations of each release. Initially there are 80 drifter pairs, but this number falls off to fewer than 30 pairs after 30 days. There also tend to be more pairs near the coast.

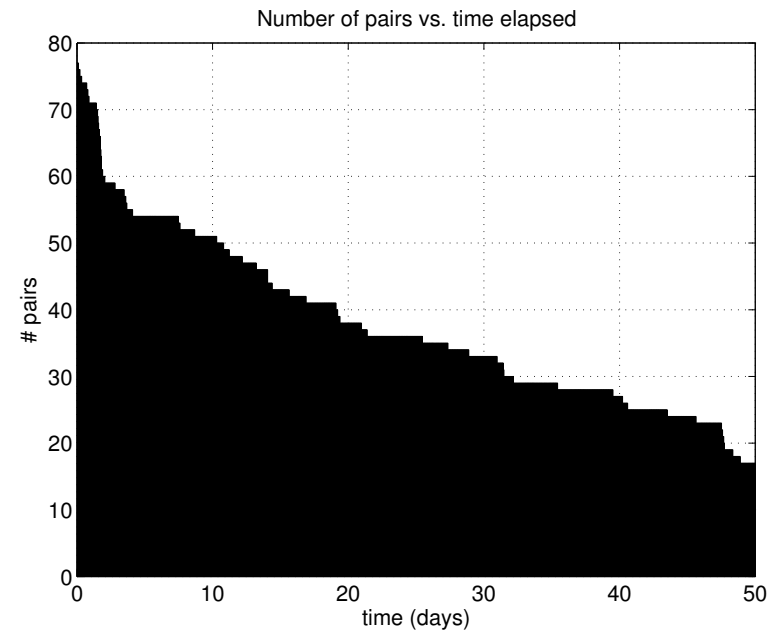

(a) Number of drifter pairs as a function of time

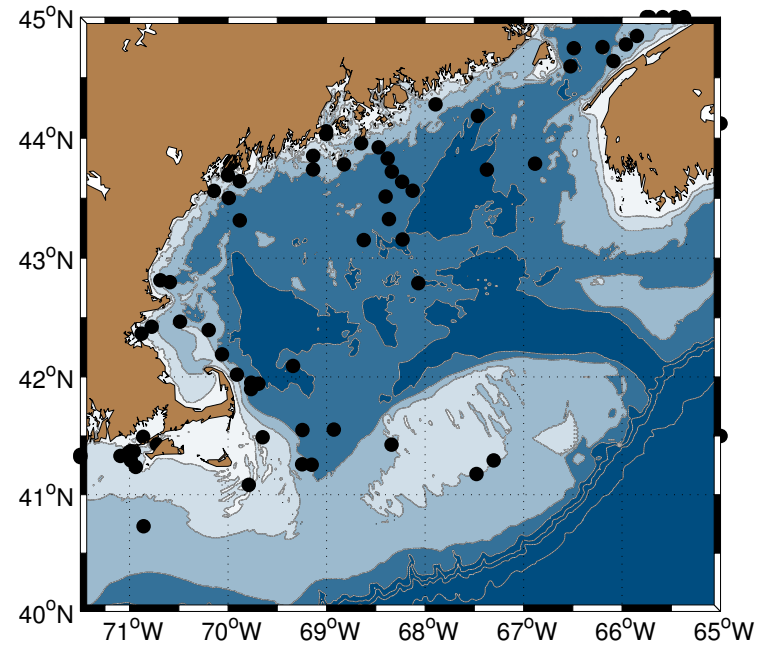

(b) Location of "release" of each drifter pair

Figure 4-11: Time (a) and space (b) distribution of drifters in the GOM observations.

For all drifter pairs, the dispersion (defined as separation distance squared) is binned as a function of time (figure 4-12). The dashed lines indicate standard error. Pair dispersion studies in other parts of the world (e.g. Koszalka and Lacasce (2009)) have observed three different regimes in dispersion curves: an initial phase from release time till about 3 days, during which the dispersion increases with increasing speed; an intermediate phase, where the dispersion increases quasi-linearly, indicating some coherent motion, and a purely dispersive phase from 8-10 days till about 20 days, 
during which the two drifters are effectively independent. The pair dispersion in the GOM may be slightly different: The data were initially low-pass filtered, so dispersion on short time scales (i.e. those less than 1 day), are perhaps misleading. Although the dispersion curve does appear to have an initial phase consistent with other studies, the low-pass filtering brings this conclusion into some question. From about 2 days to between 10 and 20 days, the dispersion increases roughly logistically, consistent with the "intermediate" phase observed in other studies. The amount of drifter pair data after 20 days is very small, and thus the pair dispersion is too noisy after about this time to make any kind of inference.

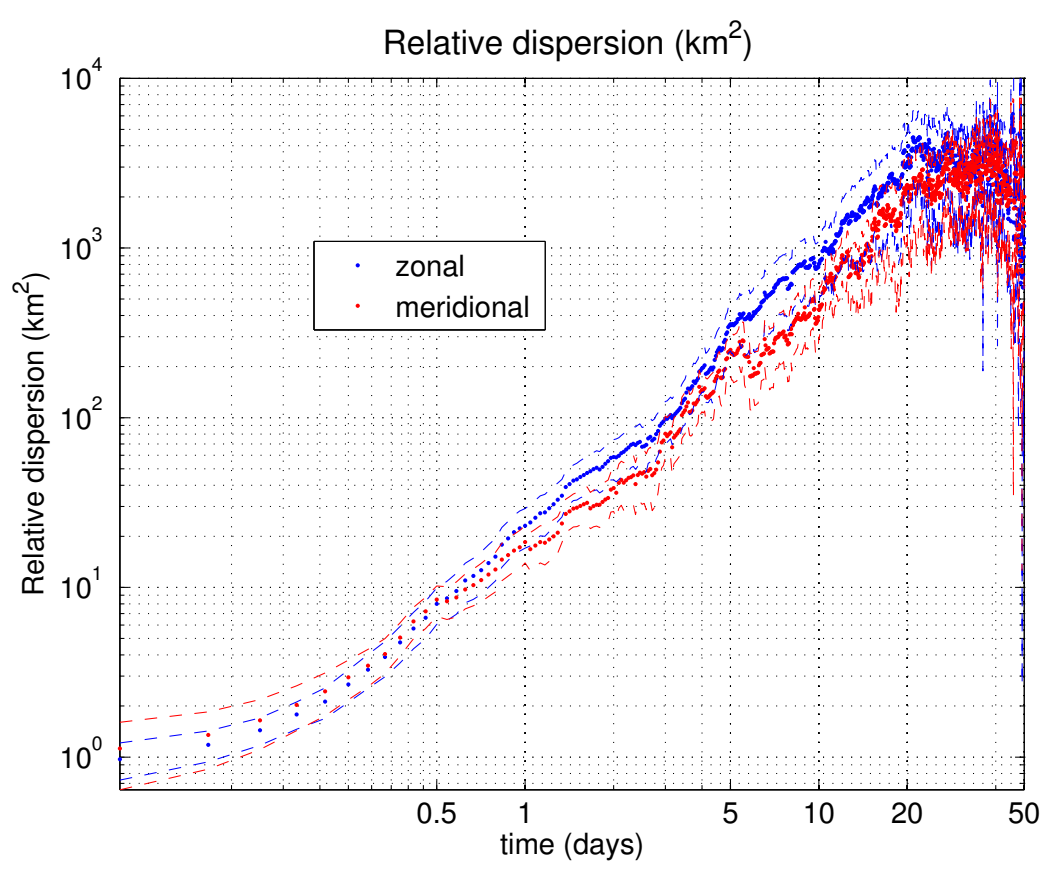

Figure 4-12: Relative dispersion as a function of time for all 80 drifter pairs in GOM data

The pair diffusivity is computed from the dispersion from equation 4.5, and is shown in figure 4-13. The first thing to notice is that both the single-particle and particle-pair estimates of $\kappa_{x x}$ are larger than their respective $\kappa_{y y}$; the diffusivity is not symmetric, but is larger in the zonal direction. This is likely a result of the effect of the coastline. Particles cannot spread through the boundary, and thus drifters are prohibited in spreading out to the north by the Maine coastline. This inhibits $\kappa_{y y}$ 
with respect to $\kappa_{x x}$. Cape Cod and Nova Scotia may inhibit some spreading in the zonal direction, however the flow south of Georges Bank is not inhibited in the zonal direction (but also limits meridional excursions of the drifters); this effect further inhibits $\kappa_{y y}$ with respect to $\kappa_{x x}$.

From about 2-3 days to just over 10 days, the pair diffusivity is roughly constant, consistent with the intermediate phase mentioned above. During this phase, the magnitude of the pair dispersion is roughly equivalent to $\kappa^{\mathrm{Eul}}$, with the zonal component larger than the meridional component. After about 10 days, $\kappa^{\text {pair }}$ increases again. The noise that dominated the dispersion in figure 4-12 also influences $\kappa^{\text {pair }}$, and thus lags longer than 15 days are not shown.

The magnitude of $\kappa^{\text {pair }}$ is comparable to that of $\kappa^{\text {Eul }}$ at lags shorter than 10 days. To compare the two, let us think about what the two different calculations are measuring. In a way, $\kappa^{\mathrm{Eul}}$ measures how differently a drifter will move when deployed at the same place at different times, while $\kappa^{\text {pair }}$ measures how a pair of drifters deployed at the same time will move relative to one another. $\kappa^{\text {Eul }}$ may include dispersion from larger scale motion, such as winds or larger-scale meanders in the coastal current, while $\kappa^{\text {pair }}$ only considers the spreading between the two drifters (and depends upon the distance between the two drifters). The inclusion of correlated motion in the calculation may cause $\kappa^{\text {Eul }}$ to overestimate the diffusivity when compared to $\kappa^{\text {pair }}$. Indeed, at short time scales (those less than about 3 days), we see that $\kappa^{\mathrm{Eul}}$ is larger than $\kappa^{\text {pair }}$. This is roughly the time scale of synoptic wind events (storms), which is when we might expect the above discrepancy between the two estimates to be most drastic.

Additional information about correlated motion in the flow can be garnered from the drifter pairs. We can directly look at the correlated motion of drifter pairs by computing the drifter separation velocity:

$$
<v^{2}(t)>=1 / N_{p} \sum_{i \neq j}\left(u_{i}(t)-u_{j}(t)\right)=2 \nu^{2}-2 \overline{u_{i} u_{j}}
$$




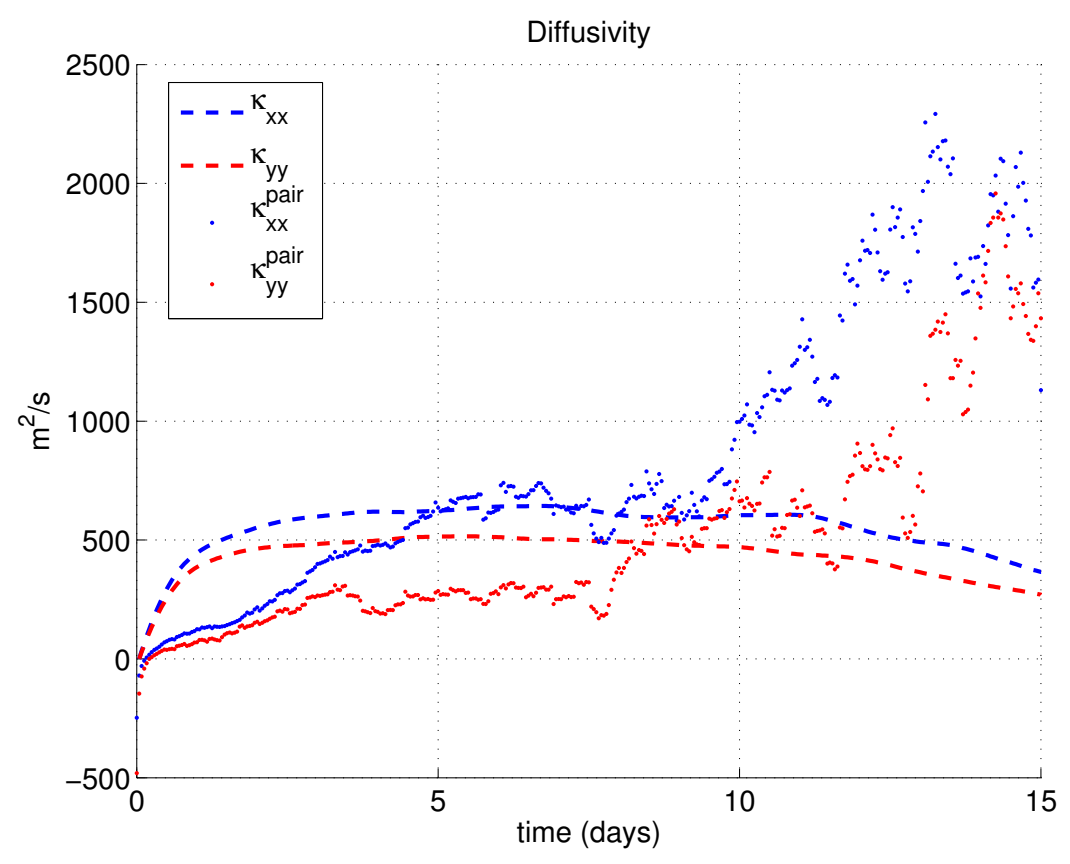

Figure 4-13: Eddy diffusivity from single particle, Eulerian mean removed (dashed lines), and particle pair (dots) statistics, $\kappa^{\text {pair }}=\frac{1}{2} \frac{d r^{2}}{d t} . \kappa>\kappa^{\text {pair }}$ because the singleparticle calculation includes correlated motion that may not necessarily be truly dispersive (e.g. wind-driven motion pushing drifters).

If the drifters are independent, then their velocities will be uncorrelated, and the mean square separation velocity will be simply equal to twice the square single-particle velocity $(\nu)$. Plotted in figure 4-14 is the mean square separation velocity as a function of time (panel a) and separation distance (panel b). In both figures, the solid black line represents the average square single-particle velocity $\left(.025 \frac{\mathrm{m}^{2}}{\mathrm{~s}^{2}}\right)$.

The data here are not sufficient to understand the time-scales associated with the correlated motion. We might expect that the mean square separation velocity asymptotes to the single-particle velocity as the drifters become uncorrelated, however it does not appear to be the case. This could indicate that we don't have sufficient information to resolve the time scales over which drifter pairs become uncorrelated, or it could mean that there is not one single time scale for the Gulf of Maine but rather a series of time scales depending upon whether one is in the coastal current, interior of the GOM, on Georges Bank, or elsewhere. 


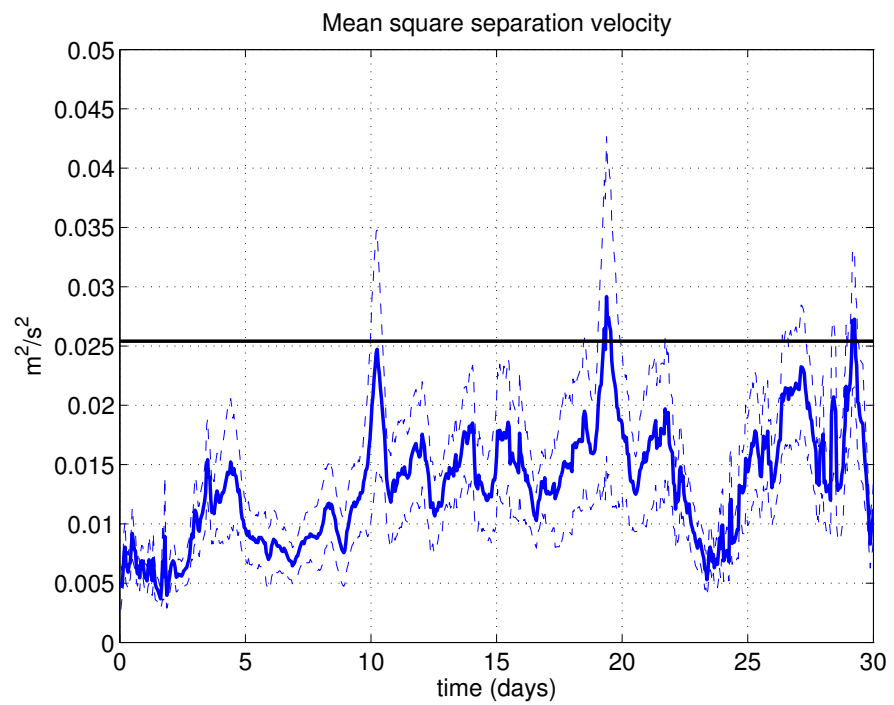

(a) Separation velocity vs time

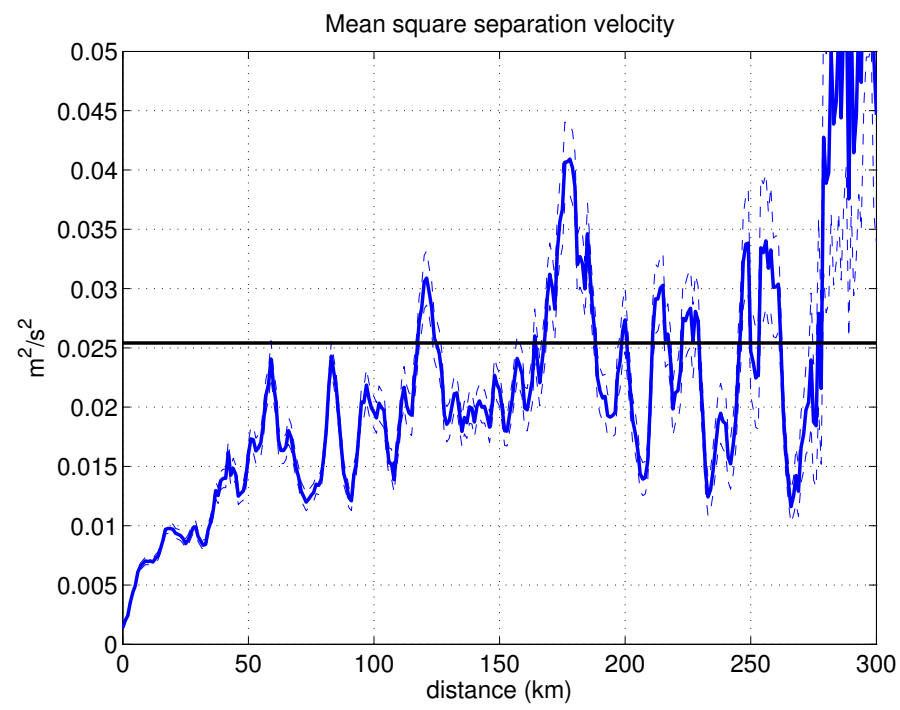

(b) Separation velocity vs separation distance

Figure 4-14: Mean square separation velocity versus time (left) and distance (right) since release.

From panel b in figure 4-14 we can determine over what spatial scales the drifter velocities are correlated. The separation velocity increases with distance until about $50-100 \mathrm{~km}$, after which is it relatively constant and equal to the single-particle velocity. This suggests that the dominant scale of the eddies is in the range of $50-100 \mathrm{~km}$. 


\subsubsection{Three sub-regions: Interior GOM, Coastal Current, and Georges Bank}

To investigate the spatial variability of $\kappa$ in the GOM, we now compute the components of the diffusivity tensor over the three different geographic bins pictured in figure 4-15. These bins correspond to what we expect are different dynamical regions:

1. Coastal Current, $C C$ : Strong current flows around the perimeter of the GOM. This bin extends from the coast to $50 \mathrm{~km}$ offshore.

2. Interior GOM: Relatively quiescent interior.

3. Georges Bank, GB: This bin is defined as all points east of $69^{\circ} \mathrm{W}$ that are

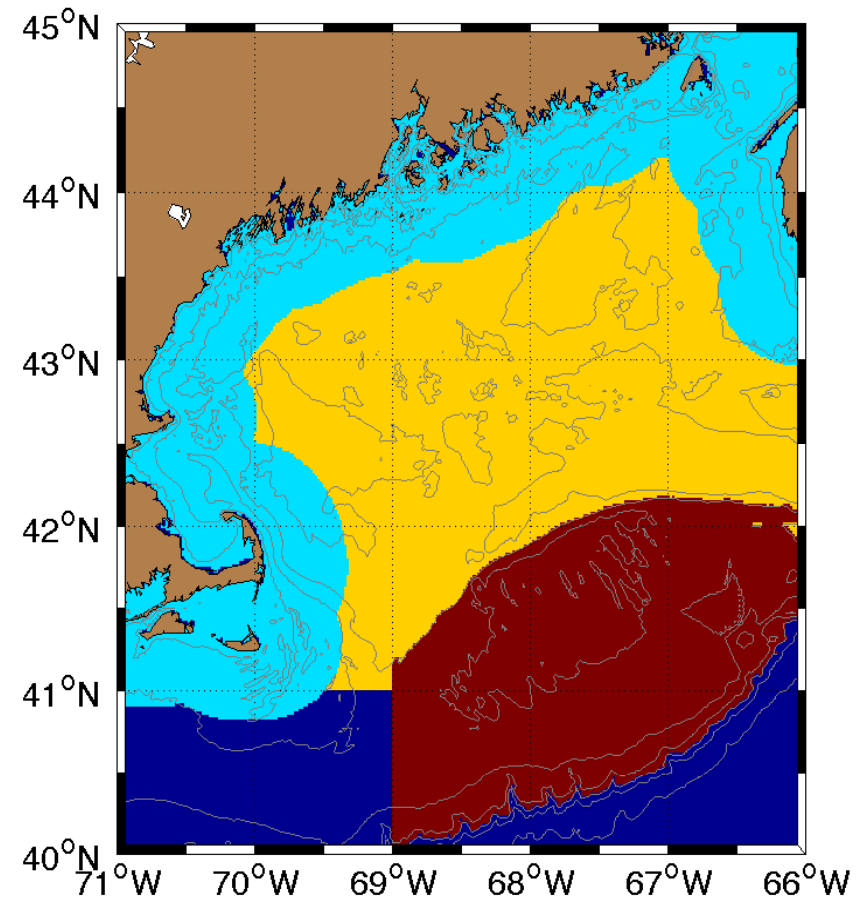

Figure 4-15: Bins for figure 4-16. Average residence times for drifters in each bin are approximately 14, 18, and 16 days for the Coastal Current (light blue), interior GOM (yellow), and Georges Bank (red) bins, respectively.

These three regions are chosen as such to be large enough so that the trajectories within each bin will be long enough to obtain good statistics, but shaped so as to 
include what we may expect are different physical regimes. There are 293, 187, and 71 drifters with segments in the CC, GOM, and GB bins, respectively. Taking only those segments within each bin, we compute the diffusivity following the same method as above, shown in figure $4-16$. Here, we only show $\kappa^{\text {Eul }}$.
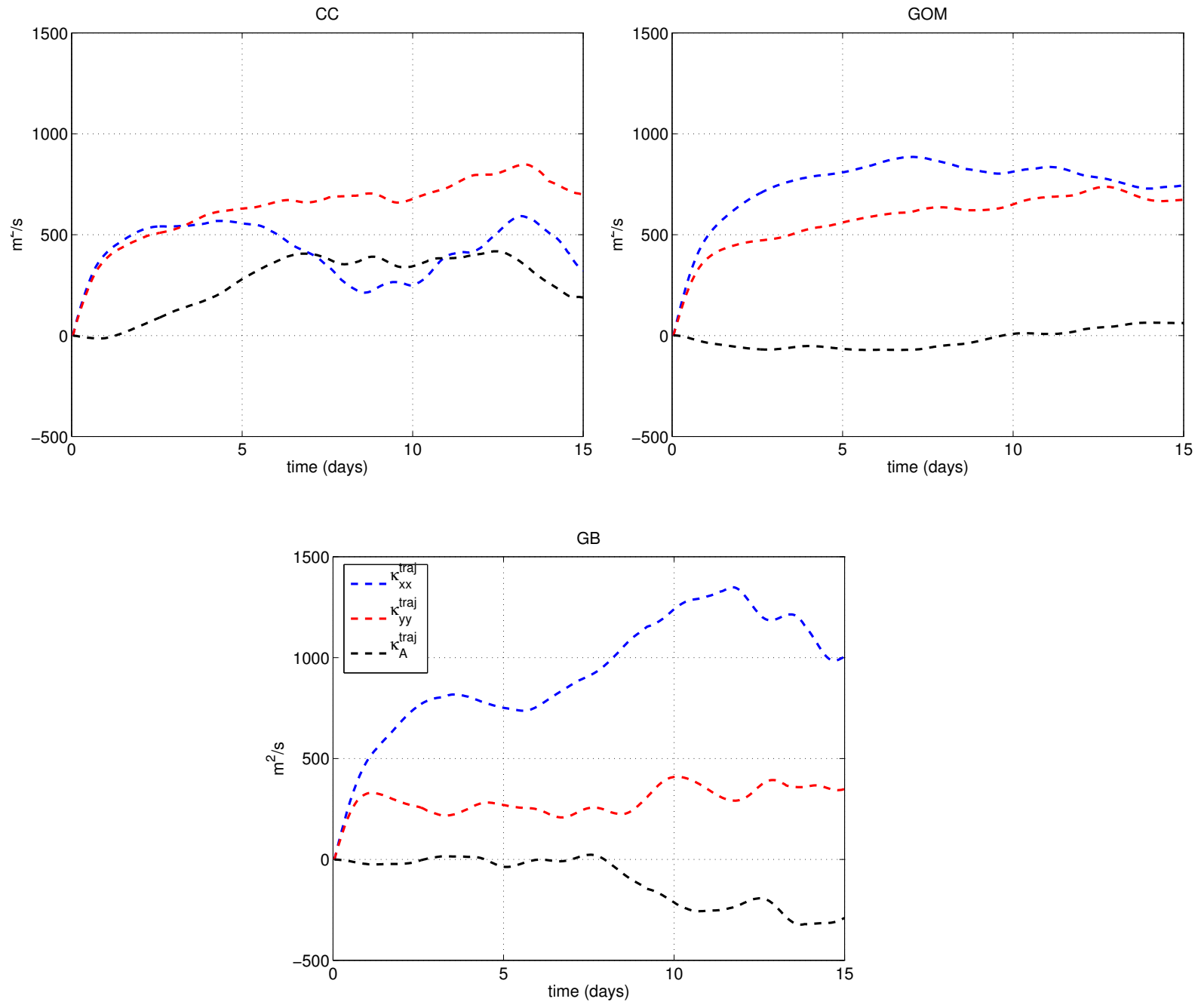

Figure 4-16: Diffusivity estimates for bins in figure 4-15. Lags are in days, $\kappa$ in $\mathrm{m}^{2} / \mathrm{s}$

It is now possible to make some inferences about the spatial variability of eddy diffusivity around the GOM. Just as in the entire GOM, the diffusivity is not isotropic. In the GOM and Georges Bank, the zonal component is larger than the meridional. The diffusivity is more isotropic in the coastal current, with the meridional component only slightly larger. Of the three regions, Georges Bank has the largest zonal 
component of $\kappa$, while the Coastal Current has the largest meridional component. The antisymmetric parts of the diffusivity tensor are positive in the Coastal Current and negative on Georges Bank, indicating the tendency for cyclonic and anticyclonic flow in those regions, respectively. In the interior GOM, there is no preference for cyclonic or anticyclonic flow.

\subsubsection{Comments on using east-north coordinates to estimate $\kappa$ in coastal regions}

In the open ocean, diffusivity estimates are often computed in a fixed east-north coordinate system, In this chapter's work, we adopted the east-north convention, however it may not be appropriate for a few reasons. In the presence of a sheared mean flow, shear dispersion may cause overestimation of $\kappa$ in the direction of the mean flow (Zhurbas and Oh (2003)). Also, the presence of the coastline will impose anisotropy in $\kappa$ simply by limiting the deviations a particle may take in the cross-shore direction. We can evaluate whether the traditional east-north coordinate system is appropriate for $\kappa$ estimates in the GOM by comparing the magnitude of the offdiagonal components of the $\kappa$ tensor to the diagonal components. We also compare the principal axes of the $\kappa$ tensor to the diagonal components, which will indicate how appropriate the east-north coordinates are. Zhurbas and Oh (2003) noted that the major principal axes of the $\kappa$ tensor may be exaggerated when compared to the minor principal axes in the presence of shear dispersion. We also check this here.

Figure 4-17 shows the individual components of the $\kappa$ tensor, as well as the major and minor axes values, averaged over the entire GOM. The off-diagonal components of $\kappa$ are small (less that half) that of the diagonal components. Also, the principal axes values are very similar to that of $\kappa_{x x}$ and $\kappa_{y y}$, indicating that the East-North coordinate frame is suitable for GOM-scale diffusivity estimates. The fact that $\kappa_{1}$ is almost twice that of $\kappa_{2}$ indicates that some shear dispersion may be occurring within the GOM, and so the estimates of $\kappa$ in the east-north coordinates might 


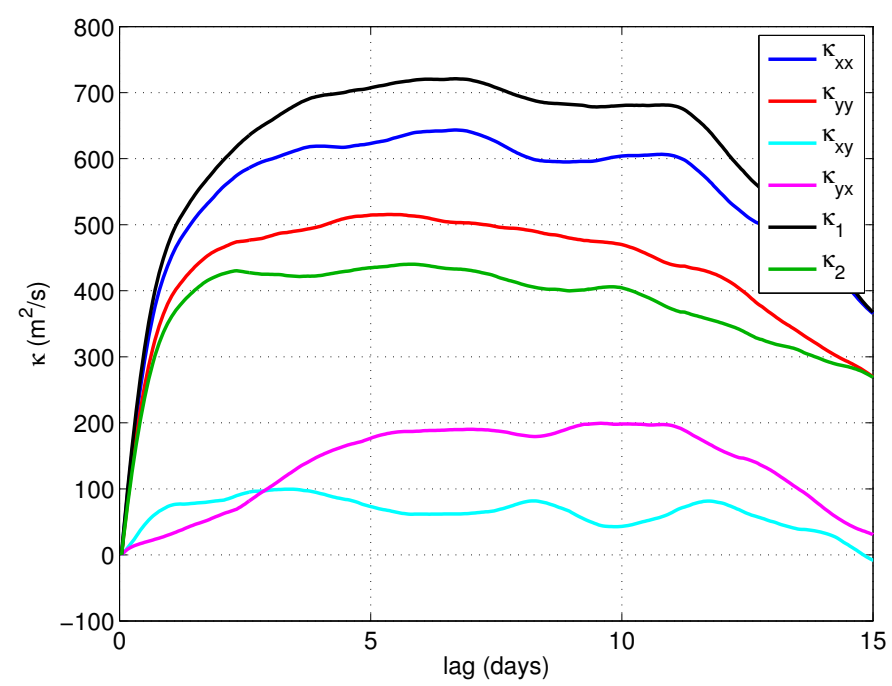

Figure 4-17: Elements of the $\kappa$ tensor, as well as principal axes values, for entire GOM

be exaggerated in the direction of the mean flow. Next let us consider the three subregions (figure 4-18):

The patterns in $\kappa$ in the GOM bin behaves similarly to the average over the entire GOM: The off-diagonal components are small compared to the diagonal, and the principal axes values are similar in magnitude to $\kappa_{x x}$ and $\kappa_{y y}$. The GB and CC bins, however, do not exhibit the same patterns. In the $\mathrm{CC}$ bin, three components of the diffusivity tensor (all except for $\kappa_{x y}$ ) are of similar magnitude, indicating that the east-north coordinate frame may not be the most appropriate here. Also, the 1st principal axes value is much larger (more than twice) the 2nd principal axes value, indicating that $\kappa_{1}$ may be over-estimating the diffusivity due to shear dispersion. This is not surprising for the CC bin, since this bin contains the horizontally-sheared coastal current.

In the GB bin, three of the four components of $\kappa$ are similar in magnitude, with $\kappa_{x x}$ more than twice the other components. The major principal axis component is very similar to $\kappa_{x x}$. Recall that there is a very strong eastward-flowing jet along the northern flank of Georges Bank in the summer time. Shear dispersion associated with this jet will tend to enhance the estimate of $\kappa$ aligned with this jet. This explains the 

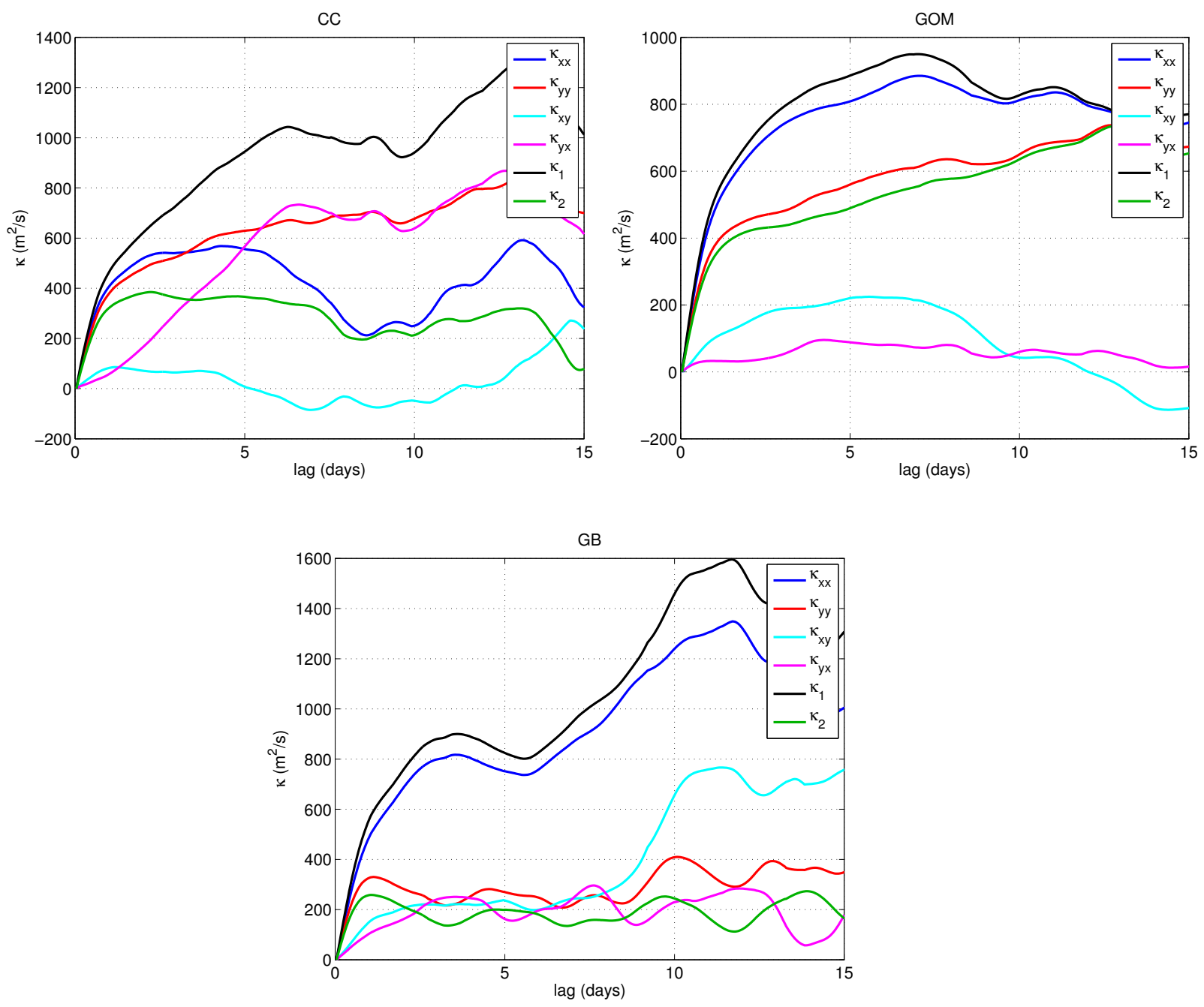

Figure 4-18: Elements of the $\kappa$ tensor, as well as principal axes values, for three subregions

enhancement of $\kappa_{x x}$ and $\kappa_{1}$ relative to the other components. In general, the choice of coordinate system utilized to compute $\kappa$ should depend upon the characteristics of the flow. For the GOM, a east-north coordinate system seems to be sufficient in the interior GOM, while on Georges Bank and along the coast, anisotropy and stronglysheared mean flows suggest an principal axes system may be more appropriate.

\subsection{Discussion and conclusion}

The major points of this work are summarized as follows: 
- Mean flow and EKE can be estimated using drifters (figure 4-2). Using a clustering method (Koszalka and Lacasce (2010)) gives smaller standard error for comparable resolution in regions of high data volume (figure 4-4).

- $\kappa$ evaluated over entire GOM by removing the trajectory mean is consistent with previous estimates (figure 4-9), but pair-diffusivities are more consistent with removing Eulerian mean (figure 4-13).

- Observations indicate that $\kappa_{y y}$ is largest in the coastal current bin, while $\kappa_{x x}$ is smallest in this bin and largest in the Georges Bank bin (figure 4-16), perhaps due to the alignment of the coastal current with the coastline or the edge of Georges Bank.

- Pair diffusivity estimates are lower than single-particle with trajectory mean removed but are consistent with single-particle with Eulerian mean removed, and contain three regimes: 0-3 days where the separation rate increases, 3-10 days where the separation rate is relatively constant, and after 10 days when the separation estimate is noisy (figure 4-13).

- Drifter pairs become decorrelated after about 10 days and 50-100km (figure 4-14)

- Effective horizontal resolution of surface drifters in the GOM is too large to evaluate dispersion and patch spreading on scales relevant to copepod patches (less than $1 \mathrm{~km}$ ) (figure 4-19). More advanced Lagrangian estimates are necessary in order to resolve those scales.

Single particle vs particle pair diffusivity estimates The single-particle statistics simply compute correlated motions in the flow. Any departures from the mean flow will be included in this calculation. Eddies are one such example, but other motion, including wind-driven and large-scale meanders in the mean flow will also be included. These motions increase the estimate of $\kappa$, but don't necessarily mix the fluid. Suppose 
there are two drifters sitting close to one another in the coastal current, when an alongshore wind begins to blow. The drifters will be advected in concert by the wind-driven flow across the coastal current. When the single-particle diffusivity is calculated, these wind-driven meanders will increase the estimate. However, since the drifters both experience the same wind-driven flow and move in concert, the distance between the drifters won't increase, and the pair diffusivity won't reflect this motion. In other words, the single-particle diffusivity estimates only the temporal decorrelation scale, while the pair-diffusivity includes the spatial and temporal decorrelation scales following a parcel of water (or a drifter pair), which is more like what a copepod patch would feel as it moves through the spatially and temporally varying flow field. In this sense, the particle pair diffusivity gives a better sense of how the a patch of copepods will disperse due to eddies in the flow, while the single-particle diffusivity more tells how a particle patch will move as a coherent unit.

What can we learn about copepod patches from drifters? Right whales (and, by inference, copepod patches) are mostly found in the southwestern Gulf of Maine in the late spring/early summer, stretching from Cape Cod through the GSC towards the northern flank of Georges Bank. The mean flow in this region is characterized by the strong coastal current jet into the region along Cape Cod, and strong flow out of the region joining the jet along the northern flank of Georges Bank. In the GSC, however, the flow is slower, less jet-like and more spread out. If a copepod patch is caught up in the coastal current flow, as it moves into this slower-moving region in the GSC, it may be easier for a right whale to locate this patch, explaining the high numbers of whales seen here. Conversely, if copepod patches are advected by the coastal current, right whales may be sitting in this region where the eddy activity is reduced and waiting for patches to be delivered to them by the coastal current.

Now let us turn to the diffusivity. More right whales are found in the $\mathrm{CC}$ bin than the either of the other two bins. Figures 4-2 and 4-3, however, show that the EKE is highest in the coastal waters of the GOM. Meanwhile, the eddy diffusivity 
shown in figure 4-16 indicates that the eddy diffusivity is actually low in the CC bin (comparable to that of the interior, and smaller than that observed on Georges Bank). Again, the EKE measures the average departure of the flow from the mean, while the eddy diffusivity reflects how efficient the flow is at destroying a patch (although the single-particle diffusivity may not necessarily be interpreted in this way, see above discussion). The high EKE in the coastal waters indicates substantial variability from the mean, but the low eddy diffusivity indicates that this motion is likely correlated in such a way that a patch may not be destroyed by this motion.

Given an estimate of the eddy diffusivity, if we make an assumption about the scale of a copepod patch, we can calculate a scale estimate of the time it would take a copepod patch to disperse. Starting with the diffusion equation for a passive tracer $C:$

$$
\frac{\partial C}{\partial t}=\kappa \nabla^{2} C
$$

Where $\kappa$ is the diffusivity. Multiply equation 4.7 by $x^{2}+y^{2}$, integrate over a volume $d V$ and rearrange to get

$$
\frac{\partial}{\partial t}\left(\frac{\int\left(x^{2}+y^{2}\right) C d V}{\int C d V}\right)=4 \kappa
$$

using $\frac{\partial}{\partial t} \int C d V=0$. From this, the diffusive time scale may be estimated as

$$
T=\frac{L_{\text {final }}^{2}-L_{\text {initial }}^{2}}{4 \kappa}
$$

where $T$ is the time scale estimate, $L_{\text {final }}$ and $L_{\text {initial }}$ are the length scales of the patch. From figure 4-13, lets take $\kappa=500 \frac{\mathrm{m}^{2}}{\mathrm{~s}}$. As described in chapter 1 , the typical scale of a copepod patch is $1 \mathrm{~km}$, and the copepod density is approximately 10x larger than the ambient density (Baumgartner and Mate (2003)). Here, we're calculating the time for a patch to spread from $1 \mathrm{~km}$ out to $10 \mathrm{~km}$, where the copepod concentration will be equal to the background concentration. With these parameters, we can estimate $T$ to be about 12 hours, which is roughly consistent with the observed temporal persistence 
of patches in the southwestern GOM (see chapter 1, M. Baumgartner, pers. comm.). This implies that

Effective resolution of the drifter data Drifters in this dataset send position fixes at irregular intervals, but they average around 2 hours, which are interpolated to 1 hourly time series for all the above analysis. The combination of the movement of the drifters due to the flow combined with the rate at which they report their positions yields an effective spatial resolution of the resulting data that depends upon the speed of the flow. The effective resolution of hourly position fixes is shown in figure 4-19. It is important to note that in regions of strong mean flow (such at the coastal current), the effective horizontal resolution of the drifter positions is about $1 \mathrm{~km}$, which is the roughly the scale of the observed copepod patches (M. Baumgartner, personal communication). In practice, the fixes occur less frequently than hourly, and the data were low-pass filtered, so the resolution is even poorer. This means that the drifter dataset is not actually resolving dispersion on the spatial scales of the copepod patches.

Instead, the work presented here can be interpreted from a few different points of view: the eddy diffusivity presented here could represent motion that would move copepod patches relative to one another, rather than individual copepods. Or, the scales measured by this data could be generating motion on smaller scales (fronts, filaments, etc.) that form, maintain, or destroy copepod patches. We simply do not have observations on small enough scales to understand how a copepod patch may be affected by eddies in the GOM. The single biggest take-home message from this work is that additional, targeted observations need to be made in the vicinity of copepod patches to understand Lagrangian motion on appropriate scales.

Most of the above work, especially the eddy diffusivity estimates, involves ensemble averaging of some sort. The uncertainty thus depends upon how many realizations of the flow are involved in the estimate. But how many realizations are necessary to make a reasonable estimate of the quantities we care about? Similarly, how many 


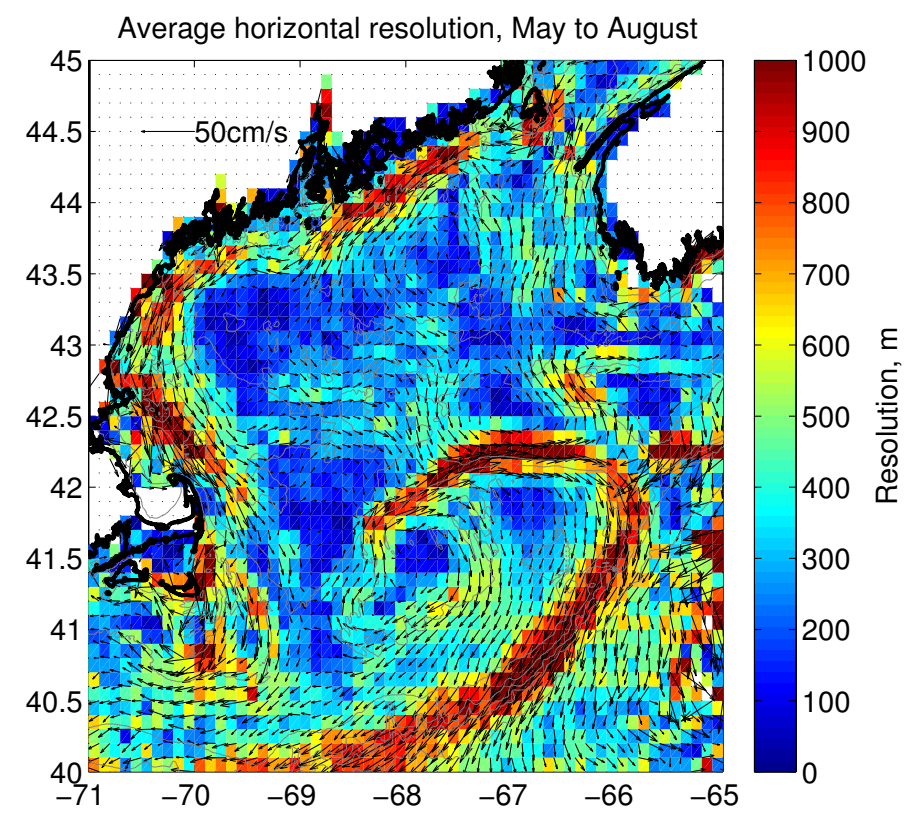

Figure 4-19: Horizontal resolution based upon mean flow and hourly drifter position fixes.

realizations are required to get a target uncertainty? These questions we cannot address with the observations, and so we turn to numerical drifter simulations. This is the topic of chapter 5 . 


\section{Chapter 5}

\section{Evaluating Lagrangian methods in the Gulf of Maine}

\section{$5.1 \quad$ Introduction}

Surface drifters have been used in many regions of the world's oceans to understand the circulation (e.g. Davis (1991), Swenson and Niiler (1996), Brink et al. (2003), Lacasce (2008)). In the previous thesis chapter, we used surface drifters to characterize the flow in the Gulf of Maine (GOM), a semi-enclosed basin on the northwest Atlantic shelf. A series of analysis techniques were used to diagnose the flow variability, which have been used in other studies (e.g. Davis (1991), Swenson and Niiler (1996), Lumpkin et al. (2002), Lacasce (2008)). This chapter evaluates the techniques themselves in order to better understand the sensitivities of each method, and to help with the interpretation of the data. Specifically, we evaluate four distinct parts of the analysis performed in chapter 4: calculation of the mean flow and eddy kinetic energy (EKE), the processing techniques used on the Lagrangian data, estimating the eddy diffusivity and its uncertainty.

Two important characteristics of the flow in any system are the time-mean and eddy kinetic energy (EKE), which can be calculated from surface drifter data. Traditional methods involve taking the processed data and averaging it over some standard 
grid to gain a picture of the mean flow. Obviously, the statistical significance of the resulting mean is dependent upon the number of different drifters passing through a particular grid cell, which can vary a great deal. A new technique (Koszalka and Lacasce (2010)) uses an adaptive binning technique, in which the shape of the averaging area changes so that roughly the same number of observations are contained within each cell. In the previous thesis chapter, we applied this new method to the GOM drifter dataset; in this chapter, we quantify the differences between the two methods.

The information collected from a Lagrangian drifter usually amounts to nothing more than a series of latitude/longitude positions spaced out in time. Taking this position data that is irregularly spaced in time and transforming it to a useful picture of the flow involves a series of processing steps, including smoothing over high-frequency oscillations (e.g. tides), interpolating in time, and computing water velocity from the positions. Each of these steps may introduce errors and/or biases to the analysis, which we investigate here.

Another important part of the flow, especially as pertains to copepod patches, is the eddy diffusivity, which is a measure of how effective the flow is at spreading a patch of tracer (or copepods) out in the fluid. There are a few methods for calculating the eddy diffusivity from surface drifters; in this chapter we compare the eddy diffusivity from model drifters with that of the observations. Also, the accuracy of these methods depends crucially on the number of different drifters in the area. For example, the accuracy of single-particle diffusivity estimates has been shown to depend to a high degree on the number of drifters used in the calculation (Klocher et al. (2012)). Using a numerical model and model drifters, we can quantify the uncertainty in diffusivity estimates given a certain number of drifters, and determine whether current observations are sufficient to make the desired inferences, or whether additional observational effort should be undertaken.

The structure of this chapter will be as follows: Sections 5.2 and 5.3 explain the data, model, and methods used in this work. In section 5.4, we compare the adaptive binning technique with the traditional binning method to quantify the differences 
between the two and their effect on the calculation of the mean flow and EKE. In section 5.5, the model, model drifters, and data are compared with one another to evaluate both the model and the processing techniques used on the drifters. In section 5.6, eddy diffusivities from the observations, model, and model drifters are compared with one another to determine sources of uncertainty/discrepancy between the various methods used, as well as evaluate the processing techniques. Finally, in section 5.7, the numerical model and model drifters are used to evaluate the uncertainties associated with calculating eddy diffusivity from drifter trajectories, which is followed by a conclusion in section 5.8 .

\subsection{The drifter data, model, and model drifters}

\subsubsection{GOM Drifter Observations}

The observational data, consisting of 363 drifters deployed in the GOM from 19882010, was described in the previous chapter. Here, we only reiterate the processing techniques, as these are evaluated in the following work. The position data, which are scattered at unevenly-spaced intervals in time, are linearly interpolated to a uniform one hour spacing. Each hourly drifter position time series is then low-pass filtered with the PL66 filter (Beardsley et al. (1985)), with a half-power of 38 hours, to remove tides and high frequency fluctuations. Sub-tidal drifter velocities were then computed by first-differencing the drifter position. Every drifter then has an associated time series of position and velocity.

\subsubsection{FVCOM simulations of the GOM}

In situ observations are supplemented by realistic simulations of the GOM circulation using the Finite-Volume Coastal Ocean circulation Model generation 1 (FVCOM generation 1, Chen et al. (2003), Chen et al. (2006)). This unstructured-grid, finite-volume, free-surface primitive equations model has a variable horizontal grid 

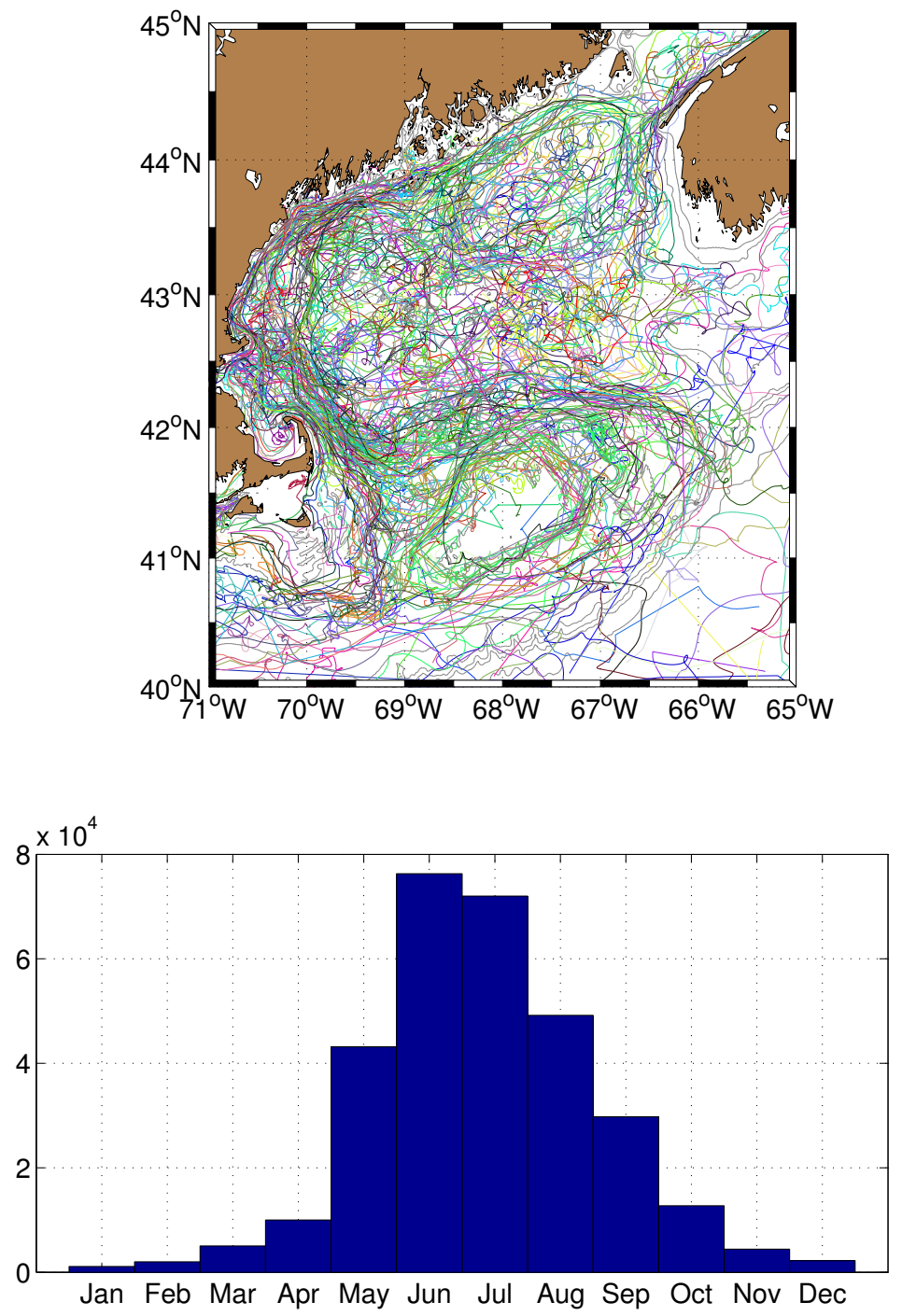

Figure 5-1: (left) All 363 drifter tracks. (right) Distribution of drifter observations by month. Wintertime data coverage is too low to allow for seasonal analysis, so only summer-time is discussed herein.

resolution that ranges from $1 \mathrm{~km}$ in the coastal waters to $10 \mathrm{~km}$ offshore. Vertically, the model has 31 sigma-levels. Closure is achieved via the Mellor-Yamada 2.5 and Smagorinsky turbulence closure schemes for vertical and horizontal turbulence, respectively. The model employs a combination of two atmospheric models (MM5 and WRF, Chen et al. (2005)), and uses the COARE 2.6 bulk algorithm (Fairall et al. (1996)) for atmospheric forcing. The model also assimilates NDBC and C-Man sur- 


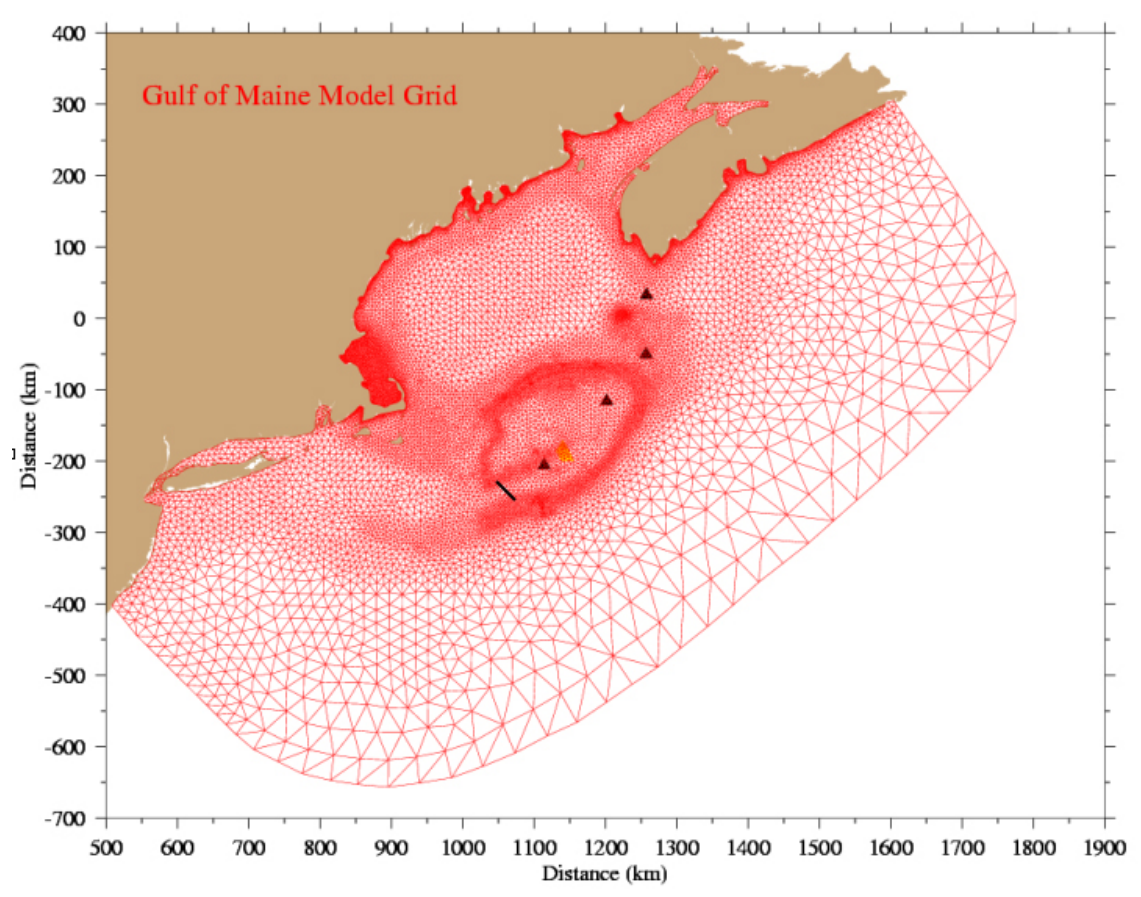

Figure 5-2: FVCOM GOM generation 1 grid

face weather data. 3-dimensional velocity, temperature, salinity, sea-surface height, and other parameters are stored hourly. One model year forced by 2011 river and surface winds is used in this analysis.

\subsubsection{Particle tracking in FVCOM}

We use an offline particle tracking model written in MATLAB (Churchill et al. (2011)). This model uses a 4th-order Runge-Kutta scheme to integrate the Lagrangian equations of motion forward in time for a series of particles. The tracker interpolates from the model's 1 hour external time step to an internal time step of 2 minutes. At each internal step, the model linearly interpolates the velocity field to the particle locations from the surrounding elements. We release 10 or 20 particles at random locations throughout the GOM every 12 hours, and track them for a period of 30 or 60 days, respectively. The tracks between May 1 and September 1 are used in the following analysis to reflect the heavy seasonal bias in the real drifter data. In total, 
this amounts to 7067 surface drifters, more than an order of magnitude larger than the number of drifters available in observational dataset. All 7067 tracks are shown in figure 5-3.

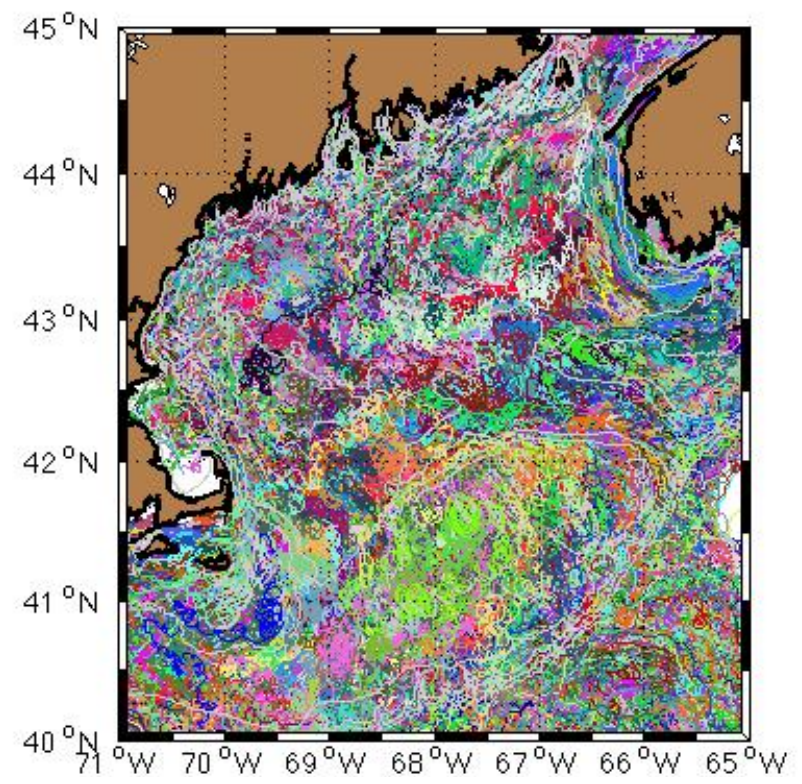

Figure 5-3: All drifter tracks from FVCOM model drifters. Individual colors represent individual drifter tracks from the model.

These drifters are processed in a similar way to that of the observations. The model records each drifter position at every model time step, which is hourly, so no interpolation in time is necessary for the model drifters. We then low-pass filter the drifter positions using the PL66 filter (Beardsley et al. (1985)), and derive water velocities from first-differences of the drifter position. As a test of first-differences, we also compute the water velocity by fitting a cubic spline to the drifter positions. 


\subsection{Methods}

\subsubsection{Comparing mean fields computed via standard grid- ding and adaptive clusters}

Two methods are employed to compute the mean velocity field and eddy kinetic energy (EKE) from surface drifters, The first method is the traditional method of dividing up the data into geographical bins and averaging all the data within a particular bin. EKE is computed from

$$
\mathrm{EKE}=\frac{1}{2}\left(<u^{\prime 2}>+<v^{\prime 2}>\right)
$$

where $u^{\prime}, v^{\prime}$ are the departures from the mean velocity field. $u, v$ and EKE are computed for $0.1^{\circ} \times 0.1^{\circ}$ bins over the entire GOM from both the observations and model drifters. The second method to compute the mean velocity field is adaptive binning, or "clustering" (Koszalka and Lacasce (2010)). In this method, velocity observations are grouped together in bins shaped such that approximately the same number of observations are contained in each bin.

We compare four different aspects of the two methods: current speed, current direction, EKE, and standard error. Standard error here is defined as S.E. $=\frac{\sigma}{\sqrt{N}}$, where $N$ is the total number of observations within a grid cell. The standard gridding and adaptive clusters do not necessarily have collocated data since the grids do not align; to compensate for this, we linearly interpolate the clusters to the standard grid, allowing for direct comparison of the two. 


\subsubsection{Computing diffusivity from single-particle and two- par- ticle statistics}

As outlined in the previous chapter, one can find the diffusivity $\kappa(\mathbf{x}, t)$ from Lagrangian particles (e.g. Davis (1991)):

$$
\kappa_{j k}(\mathbf{x}, t)=-<v_{j}^{\prime}\left(t_{0} \mid \mathbf{x}, t_{0}\right) d_{k}^{\prime}\left(t_{0}-t \mid \mathbf{x}, t_{0}\right)>
$$

where the notation $d_{k}^{\prime}\left(t \mid \mathbf{x}, t_{0}\right)$ indicates the displacement at time $t$ of a particle at position $\mathbf{x}$ at time $t_{0}$, and $\langle>$ indicates an ensemble average of particles released at different $t_{0}$, and $u^{\prime}$ and $v^{\prime}$ are the departures from the Eulerian and Lagrangian velocities, respectively, and $d^{\prime}$ is the departure of the Lagrangian displacement from the mean Lagrangian displacement $D$ (calculated as the displacement due to the mean velocity). Again, we compute diffusivity from equation 4.3 following the method outlined by Swenson and Niiler (1996). In the previous chapter, we computed the single-particle diffusivity for the observations. In this chapter, we compute the diffusivity for the model drifters, which we then compare to the observations.

We also compute the two-particle dispersion and diffusivity for the model drifters from:

$$
\begin{gathered}
<\Delta x^{2}>=\sum_{i, j}\left(x_{i}-x_{j}\right)^{2} \quad\left\langle\Delta y^{2}>=\sum_{i, j}\left(y_{i}-y_{j}\right)^{2}\right. \\
\kappa_{\text {pair }(x, y)}=\frac{1}{2} \frac{d}{d t}<\Delta(x, y)^{2}>
\end{gathered}
$$

where $i, j$ are the indices of individual drifters deployed in the same location at the same time. The differences between the model drifter diffusivities and that of the observations could be due to a few different sources, including a discrepancy in the numbers and years measured (the observations are scattered over more than 20 years and involve fewer than 400 drifters, while the model drifters are all from a simulation of the 2011 GOM circulation, involving more than 7000 drifters), differences between the model physics and reality (in particular, limits of the model grid resolution and forcing 
not resolving important processes), or limitations of the model's boundary conditions (most namely, the offshore boundary condition, which in this implementation does not include a realistic Gulf Stream).

\subsubsection{Error estimates by bootstrapping}

As noted in the introduction, Klocher et al. (2012) showed that the diffusivity computed from drifters in the Southern Ocean depends directly on the number of drifters used in the computation. We wish to know whether the number of drifters in the GOM is sufficient to discern spatial variability in $\kappa$ as well as the asymmetry in the $\kappa$ tensor. For this, we can use the model drifters.

To compute the error on the different diffusivity estimates, a bootstrapping technique is used. Given a subset of $N$ model drifters $(N=50,150,400$, and 1000), we can repeatedly calculate the diffusivity, using either of the two methods above. For each of the different $N$, we repeat the calculation 100 times, which yields a distribution about $\kappa$ for each $N$. The error is then taken to be twice the standard deviation of the distribution, which encompasses between $5 \%$ and $95 \%$ of the solutions, given $N$ drifters, assuming a normal distribution. This we will use to determine the uncertainty on $\kappa$ given the number of drifters in the observational dataset is roughly 400.

\subsubsection{Derivation of $\kappa_{G F}$}

The above are the two main methods used to determine $\kappa$ from surface drifters. In a numerical model, however, it is possible to use Green's functions to define the eddy diffusivity. The following is paraphrased from course notes from MIT course 12.820: Turbulent motions in the atmosphere and oceans (Glenn Flierl). Beginning with the advective-diffusive equation for a passive tracer $C$, the flow is split into mean and fluctuating components to get an equation for the perturbation concentration field 
$C^{\prime}$ :

$$
\left[\frac{\partial}{\partial t}+\left(\bar{u}+u^{\prime}\right) \cdot \nabla-\kappa \nabla^{2}\right] C^{\prime}=-u^{\prime} \cdot \nabla \bar{C}+\nabla \cdot \overline{u^{\prime} C^{\prime}}
$$

where $\kappa$ is now the sub-gridscale diffusion (which is assumed small), and $\overline{u^{\prime} C^{\prime}}$ represents the eddy flux. Define the average as the ensemble mean of many different realizations of the flow. For each ensemble member, we can define the Green's function as

$$
\left[\frac{\partial}{\partial t}+\left(\bar{u}+u^{\prime}\right) \cdot \nabla-\kappa \nabla^{2}\right] G^{\prime}\left(\mathbf{x}, t \mid \mathbf{x}^{\prime}, t^{\prime}\right)=\delta\left(\mathbf{x}-\mathbf{x}^{\prime}\right) \delta\left(t-t^{\prime}\right)
$$

This can be used to solve for the perturbation concentration field $C^{\prime}$ :

$$
\begin{aligned}
C^{\prime}(\mathbf{x}, t)=-\int d \mathbf{x}^{\prime} d t^{\prime} G^{\prime}\left(\mathbf{x}, t \mid \mathbf{x}^{\prime}, t^{\prime}\right) u_{j}^{\prime}\left(\mathbf{x}^{\prime}, t^{\prime}\right) \frac{\partial}{\partial x_{j}^{\prime}} \bar{C}\left(\mathbf{x}^{\prime}, t^{\prime}\right) & \\
& +\int d \mathbf{x}^{\prime} d t^{\prime} G^{\prime}\left(\mathbf{x}, t \mid \mathbf{x}^{\prime}, t^{\prime}\right) \frac{\partial}{\partial x_{j}^{\prime}} \overline{u_{j}^{\prime} C^{\prime}}
\end{aligned}
$$

which can be used to find $u^{\prime} C^{\prime}$ :

$$
\begin{aligned}
\overline{u_{i}^{\prime}(\mathbf{x}, t) C^{\prime}(\mathbf{x}, t)}=-\int d \mathbf{x}^{\prime} d t^{\prime} \overline{u_{i}^{\prime}(\mathbf{x}, t) G^{\prime}\left(\mathbf{x}, t \mid \mathbf{x}^{\prime}, t^{\prime}\right) u_{j}^{\prime}\left(\mathbf{x}^{\prime}, t^{\prime}\right)} \frac{\partial}{\partial x_{j}^{\prime}} \bar{C}\left(\mathbf{x}^{\prime}, t^{\prime}\right) \\
+\int d \mathbf{x}^{\prime} d t^{\prime} \overline{u_{i}^{\prime}(\mathbf{x}, t) G^{\prime}\left(\mathbf{x}, t \mid \mathbf{x}^{\prime}, t^{\prime}\right)} \frac{\partial}{\partial x_{j}^{\prime}} \overline{u_{j}^{\prime} C^{\prime}}
\end{aligned}
$$

Now, ignore subgridscale diffusion (i.e. $\kappa \frac{\partial^{2}}{\partial x_{j}^{2}} C_{j}^{\prime}<<1$ ) and solve equation 5.4 with

$$
G^{\prime}\left(\mathbf{x}, t \mid \mathbf{x}^{\prime}, t^{\prime}\right)=\delta\left(\mathbf{x}-\mathbf{X}\left(t^{\prime} \mid \mathbf{x}, t\right)\right)
$$

where $\mathbf{X}$ is the position of a parcel at time $t^{\prime}$ arriving at point $\mathbf{x}$ at time $t$ (a backwardsin-time trajectory). Assuming that $\bar{C}$ varies slowly in space and time,

$$
u_{i}^{\prime}(\mathbf{x}, t) C^{\prime}(\mathbf{x}, t)=-\int d t^{\prime} \overline{u_{i}^{\prime}(\mathbf{x}, t) u_{j}^{\prime}\left(\mathbf{X}\left(t^{\prime} \mid \mathbf{x}, t\right), t^{\prime}\right)} \frac{\partial}{\partial x_{j}} \bar{C}(\mathbf{x}, t)=-\kappa_{G F} \frac{\partial}{\partial x_{j}} \bar{C}\left(\mathbf{x}^{\prime}, t^{\prime}\right)
$$


where we've dropped the last term from equation 5.6. The tensor

$$
\kappa_{G F}=\int d t^{\prime} \overline{u_{i}^{\prime}(\mathbf{x}, t) u_{j}^{\prime}\left(\mathbf{X}\left(t^{\prime} \mid \mathbf{x}, t\right), t^{\prime}\right)}
$$

represents the effective eddy diffusivity, analogous to the single and pair diffusivity.

This method is useful for the following because it provides an independent estimate of the eddy diffusivity which can be used to validate (or invalidate) the other approaches.

\subsection{Standard gridding vs. adaptive clusters}

In chapter 4, we evaluated the summertime mean flow and EKE from a drifter dataset on a standard grid and using adaptive clusters. In this section, we further quantify the differences between the adaptive clusters and the standard grid. The mean velocity field and EKE for all drifter observations in the Gulf of Maine is shown for the standard $0.1^{\circ} \times 0.1^{\circ}$ grid and the adaptive clusters, as well as the standard errors of the velocity estimates in figure 5-4 (reproduced from the previous chapter).

Perhaps more interesting are the standard errors associated with the averaging. Standard error is defined here as S.E. $=\frac{\sigma}{\sqrt{N}}$, where $\sigma$ is the standard deviation of members in the bin and $N$ is the total number of observations in the bin. In the gridded fields, the standard errors are naturally lower in bins with many observations (namely the bins close to shore, in the vicinity of the coastal current). The goal of the clustering approach is to standardize the number of observations in each bin. This would mean the standard error in each bin would depend solely on the standard deviation of the observations. Averaged over the entire domain, the standard error in the gridded domain is almost a factor of two larger than that of the clusters.

Figure 5-5 depicts differences in the velocity, EKE, and standard error fields between the clustered and gridded fields. To compute the differences, the clusters were linearly interpolated to the grid locations. In all the figures positive differences indicate that the gridded values are higher than the clusters. The magnitude of the 

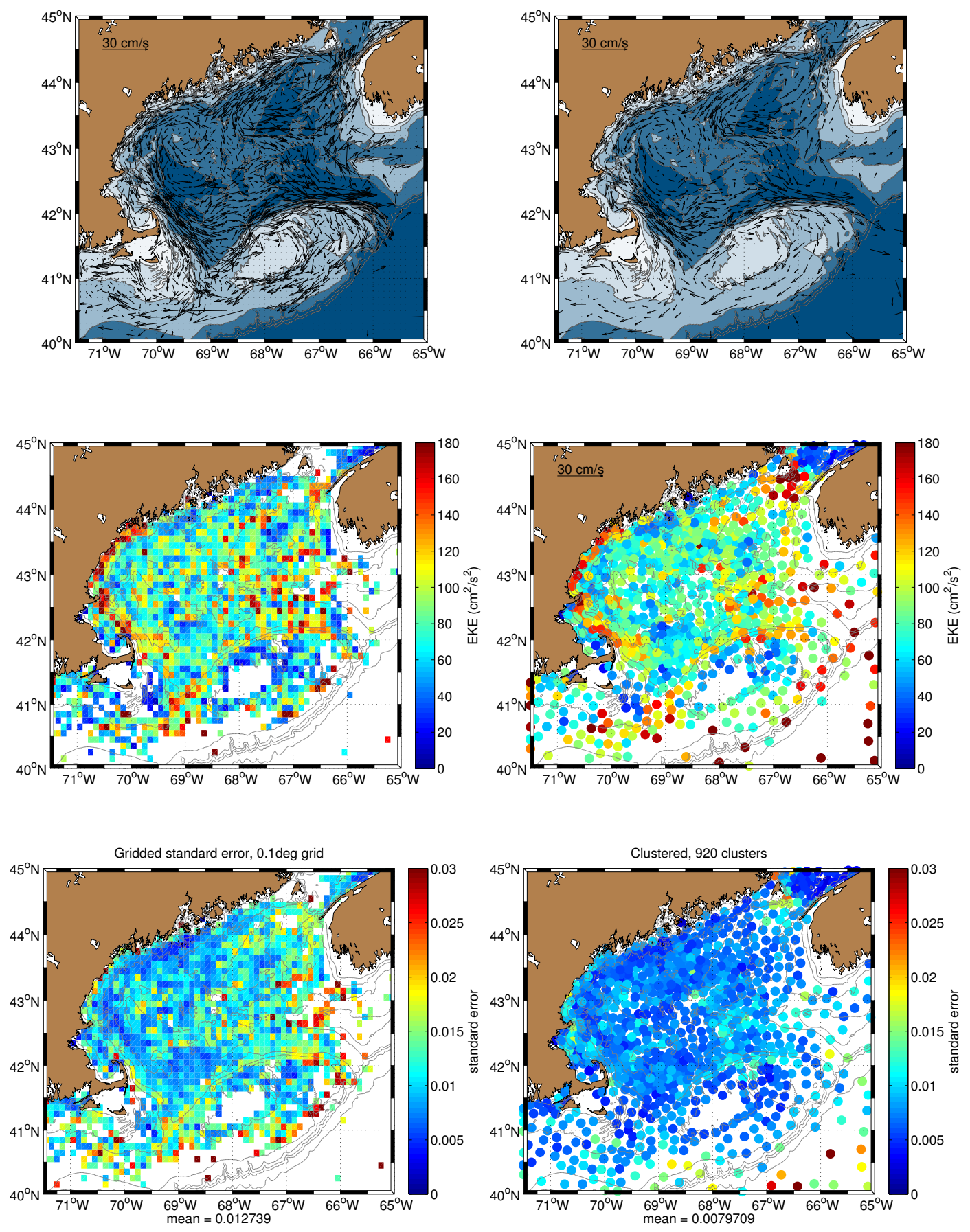

Figure 5-4: Mean velocity, EKE, and standard error from surface drifters on $0.1^{\circ} \times 0.1^{\circ}$ bins and adaptive clusters (from previous chapter).

surface velocity is very similar between the gridded and clusters in the location of the strong flow (northern flank of Georges Bank and coastal current), however, in 

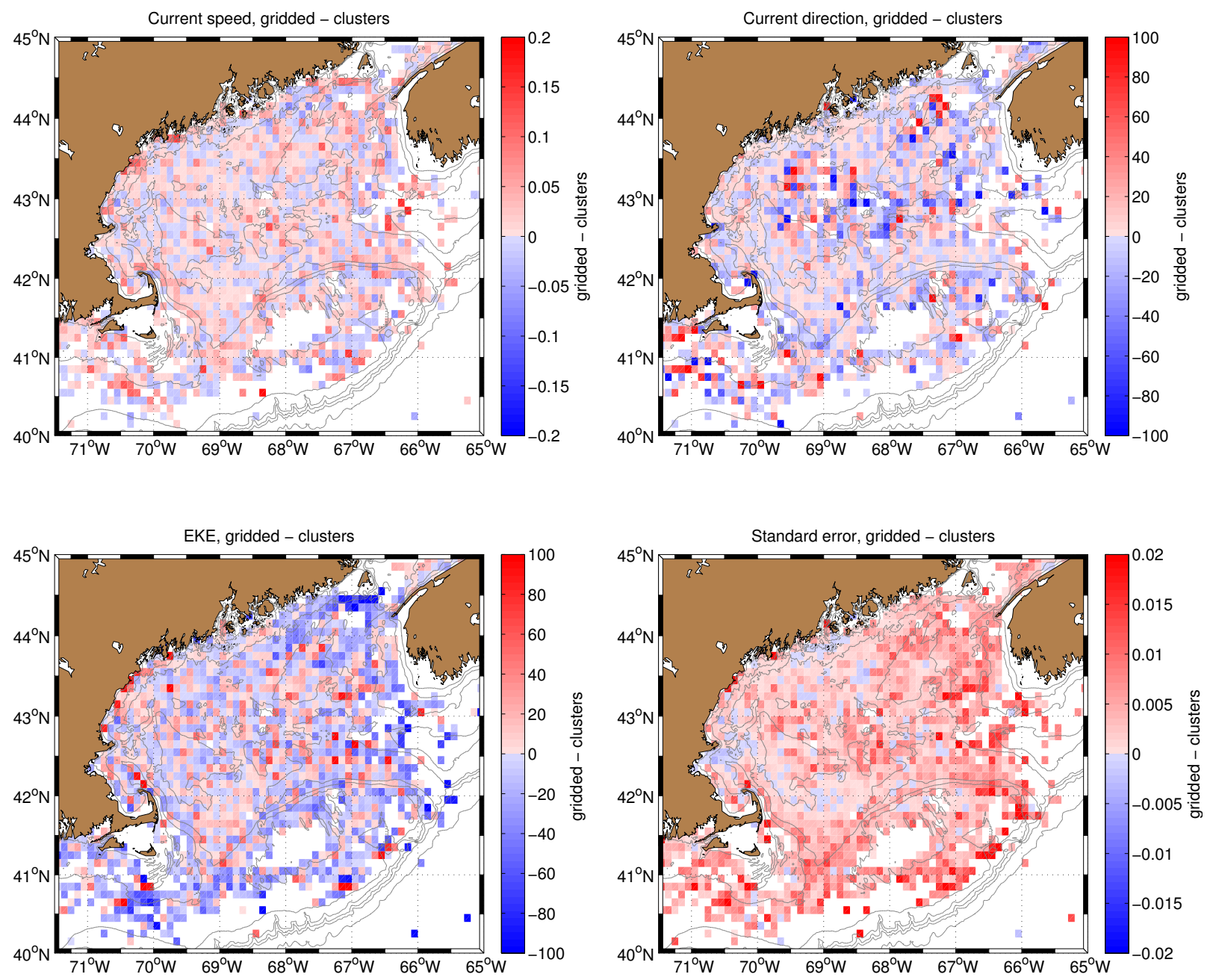

Figure 5-5: Difference plots (clusters interpolated to standard grid locations)

the interior the differences are larger, and mostly the gridded speed is higher. This is likely because the clusters average over a slightly larger area, and thus the average velocities are slightly smaller. Similarly, the direction of the flow is similar in the vicinity of strong flow, but differences get larger where the flow is weaker. The EKE is slightly different: the gridded EKE is much smaller near the mouth of the Bay of Fundy and on the shelf south of the Gulf of Maine, and larger in the interior GOM. The most interesting is the standard error, which is smaller in the clusters over almost the entire GOM, except for a small region near Cape Ann. The lower error and higher statistical significance indicate that the adaptive clustering approach is preferable for 
computing mean velocity and EKE fields from drifter data.

\subsection{Evaluating the model, model drifters, and ob- servational processing techniques}

In this section, we compare the model, model drifters, and observations in order to evaluate the different techniques used to process Lagrangian data. The FVCOM model has been validated against observations in other work (e.g. Chen et al. (2005)), however the model drifter tracks have not. There are two things to evaluate here:

1. How well the model drifters compare with the observations. This is an extension of the model-data comparison carried out in work such as Chen et al. (2005). Differences between the model and observations may indicate that the model physics are deficient in some way, or that the processing techniques used on the drifters bias the data somehow.

2. How well the model drifters compare with the model. This tests the validity of the particle tracking model and evaluates the processing steps that are implemented upon the drifter data.

This analysis is crucial because it is difficult to understand differences between the Lagrangian observations in chapter 4 with the traditional Eulerian picture of ocean circulation; comparing the Eulerian model with Lagrangian model drifters can help to understand these differences.

Differences between model, model drifters, and observations could be due to a number of sources, including discrepancies between model runs and observational years (model represents conditions in 2011, while observations are extended over year 1988-2010), steps in drifter processing that introduce uncertainty in the position or velocity (interpolation, de-tiding), or uncertainty in the particle tracking code (time or space interpolation, time stepping). The following attempts to evaluate which of these

sources of uncertainty are most egregious. We have divided this section into several 
subsections that test several different components of the analysis: Computation of the mean flow and EKE, the spatial interpolation utilized in the particle tracking algorithm, the low-pass filtering of the drifter positions to remove high-frequency fluctuations, and the computation of drifter velocity by first-differencing the drifter position.

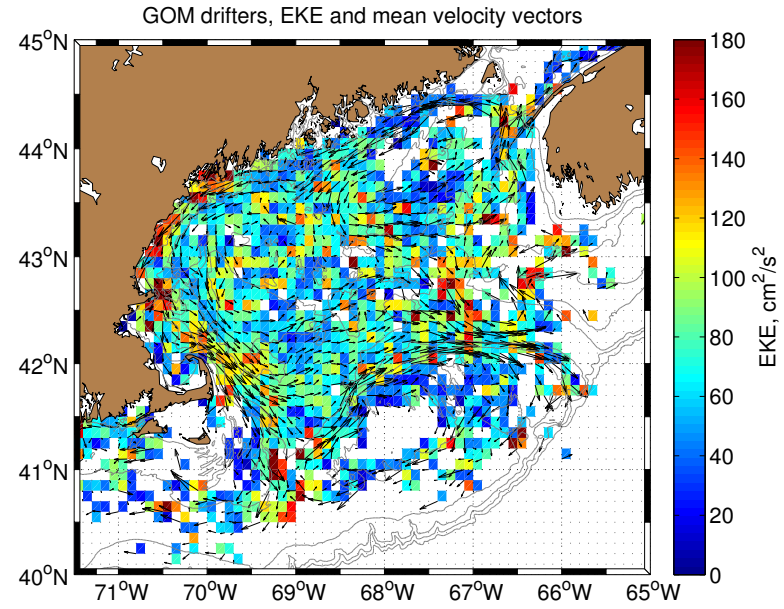

(a) GOM drifters

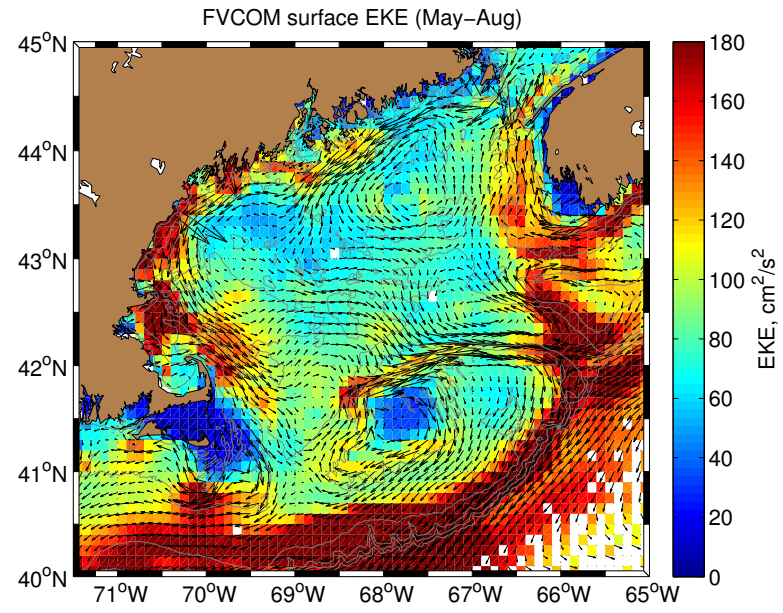

(b) FVCOM drifters gridded

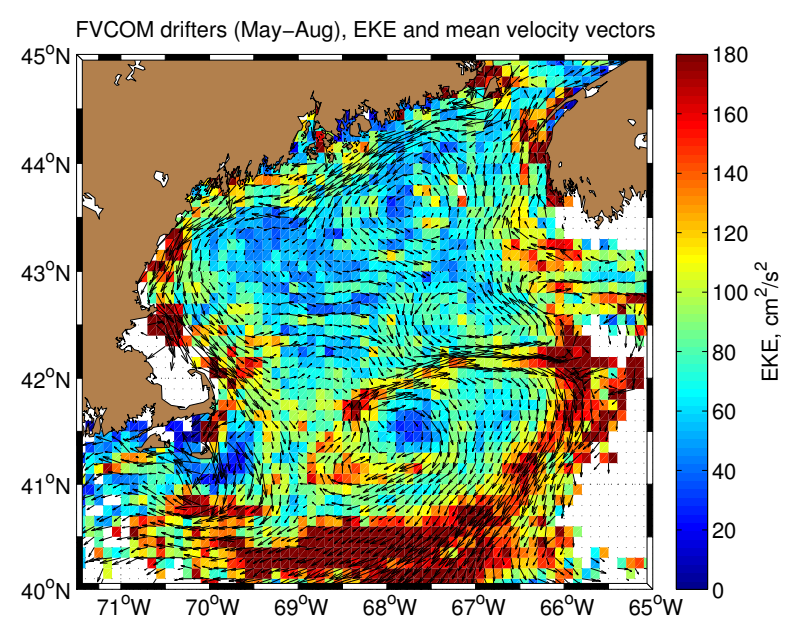

(c) FVCOM gridded

Figure 5-6: Observational (left), model (center) and model drifter (right) EKE and mean flow for the summer (May-August) months. 


\subsubsection{Mean flow and EKE differences}

In this section we compare the mean flow and EKE from the model, model drifters, and observations to see if any biases are present that must be accounted for. Figure 5-7 shows the surface velocity magnitude, direction, and EKE difference, respectively, between the model drifters and observations, and model and model drifters. The differences between the model and observations closely resembles the differences between the model drifters and observations, and are thus not shown. The spatial structure of differences between the model, observations, and model drifters can be diagnosed, most notably:

- The velocity magnitude difference between model drifters and observations do not exhibit systematic spatial biases, indicating good agreement between the model and observations.

- The velocity direction difference between the model drifters and observations, especially in the vicinity of high speed flow (e.g. coastal current) is small. The large direction difference north of Georges Bank is due to a slight discrepancy of the location of the mid-GOM current which flows from the coast of Maine directly to the Northern Flank, short-circuiting the western GOM.

- EKE differences between model and observations are largest in vicinity of coastal current and northern flank of Georges Bank.

- Differences between model and model drifter velocity magnitude and direction are mostly small and exhibit no coherent spatial structure.

- Model drifters underestimate EKE over most of GOM (except on Nantucket Shoals and Georges Bank)

The discrepancies between model drifter and observationally derived EKE appears to be somewhat correlated with the magnitude of EKE (larger errors where EKE magnitudes are larger), which may be indicative of a bias in either the model 

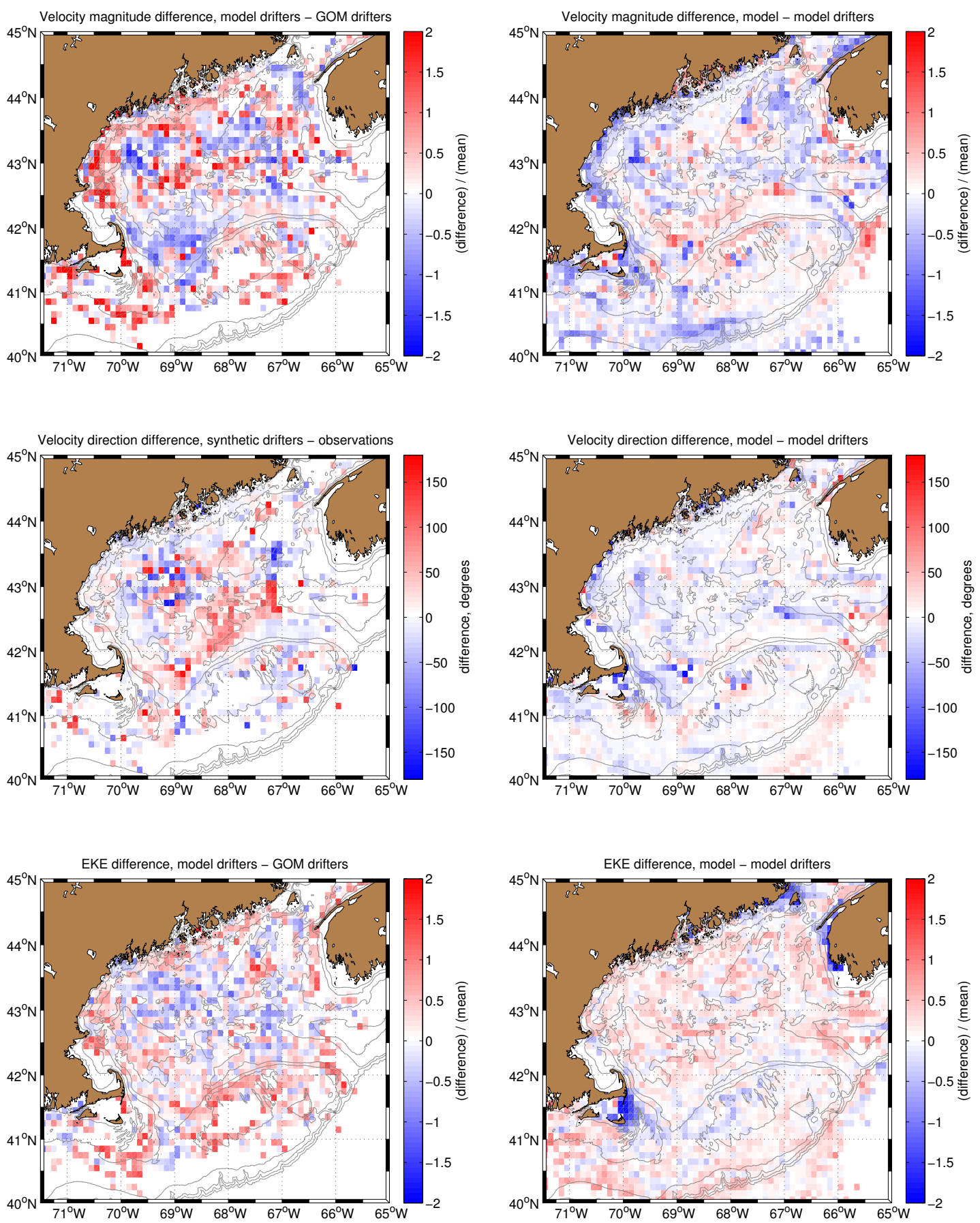

(e) model drifters - GOM drifters

(f) model - model drifters

Figure 5-7: Differences between velocity magnitude (top), direction (middle), and EKE (bottom) between model drifters and observations (left) and model and model drifters (right) 

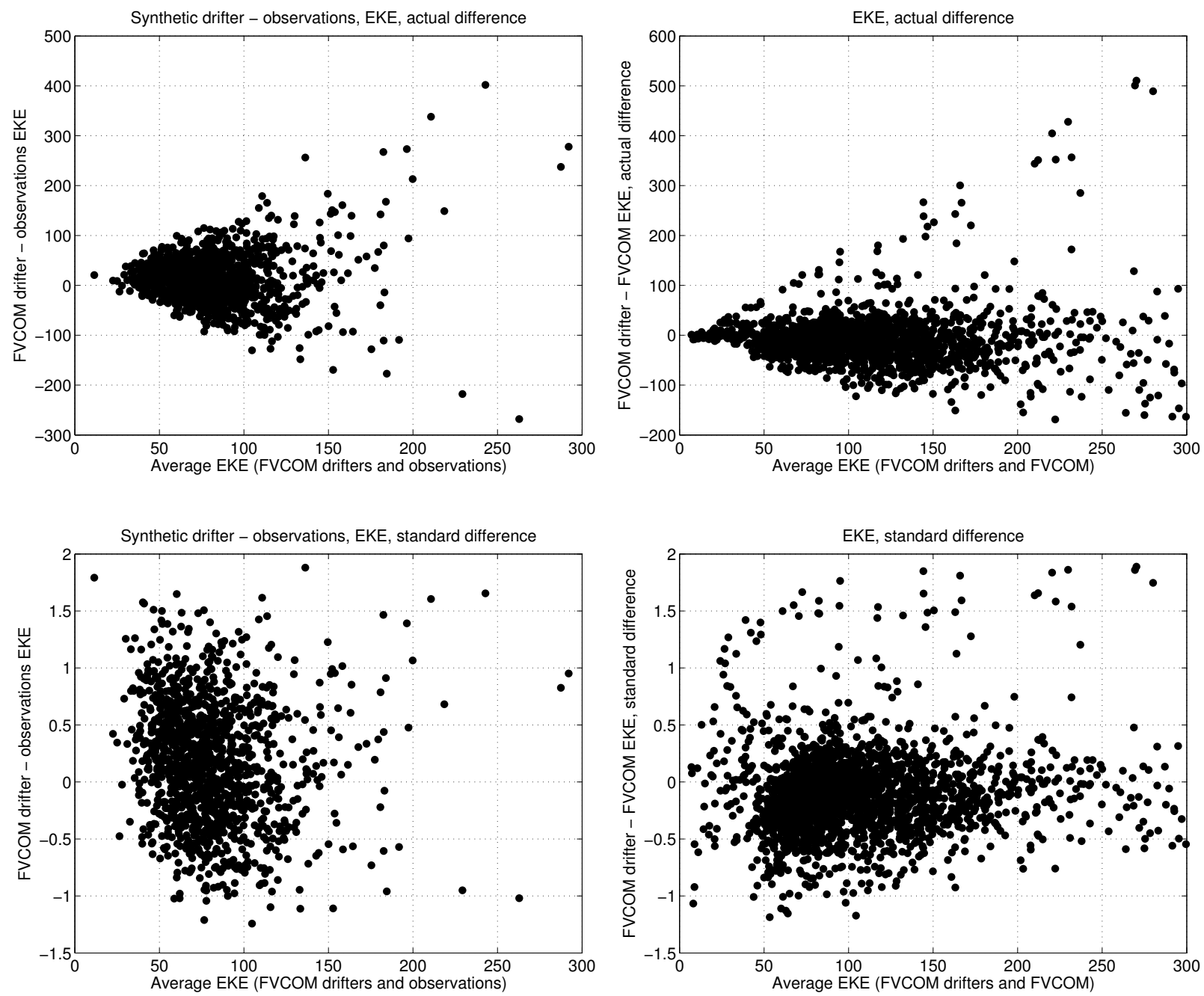

Figure 5-8: model drifters - observations vs mean (left) and FVCOM - model drifters (right), EKE

or observations. Plotted in figure 5-8 are the EKE differences between model, model drifters, and observations as a function of the magnitude of the EKE. From these figures, we can see that the difference between the model drifters and observations does indeed increase as the magnitude of the EKE increases, but the standard difference (defined as difference/mean) does not.

Interestingly, the EKE difference between the model and model drifters is almost uniformly positive (model EKE is larger), which suggests that there is some step in the calculation of the drifter tracks that causes this discrepancy. Is the source of the discrepancy a step in the processing, or due to the particle-tracking algorithm, or 
some combination? Recall that the model drifters were processed in the exact same way as the observations. If the processing is the culprit, this could have implications for how observational drifter tracks should be interpreted; conversely, if the particletracking is to blame, then perhaps a more accurate method may be employed in future studies. In the following subsections, we focus on the spatial interpolation employed in the particle tracking model and the processing steps taken to derive velocity from the drifter positions to determine which introduces the most uncertainty in the calculations. Although the differences between the model and model drifters are small over most of the GOM, the next few sections attempt to discern where the bias may come from.

\subsubsection{Testing the particle tracking spatial interpolation}

One of the steps of the particle tracking is interpolating the model velocity to the individual drifter positions. Interpolation inherently introduces some error, which is unavoidable. However, if the interpolation introduces a bias, such that the interpolated velocity is uniformly larger or smaller than the model, then the model drifters will also have this bias. In this section, we test whether any systematic uncertainty or bias is introduced by the interpolation.

To test whether the scheme in the model introduces such a bias, we compare the velocity interpolated to individual particle locations with the velocity in the element hosting each individual particle. To make the comparison, we compute the kinetic energy $\left(\frac{1}{2}\left(u^{2}+v^{2}\right)\right.$, rather than the EKE as in the previous section) which is shown for a single model snapshot in figure 5-9. Here, a single time step of the model is interpolated to the locations of particles spread throughout the GOM. The KE magnitude is nearly identical between the two and the differences are not spatially coherent, indicating that the spatial interpolation used in the particle tracking algorithm is likely not a source of the bias shown in figure 5-7.

The KE computed here is different from what is computed in previous sections. Here, because we want to focus on any possible bias due to the particle tracking 

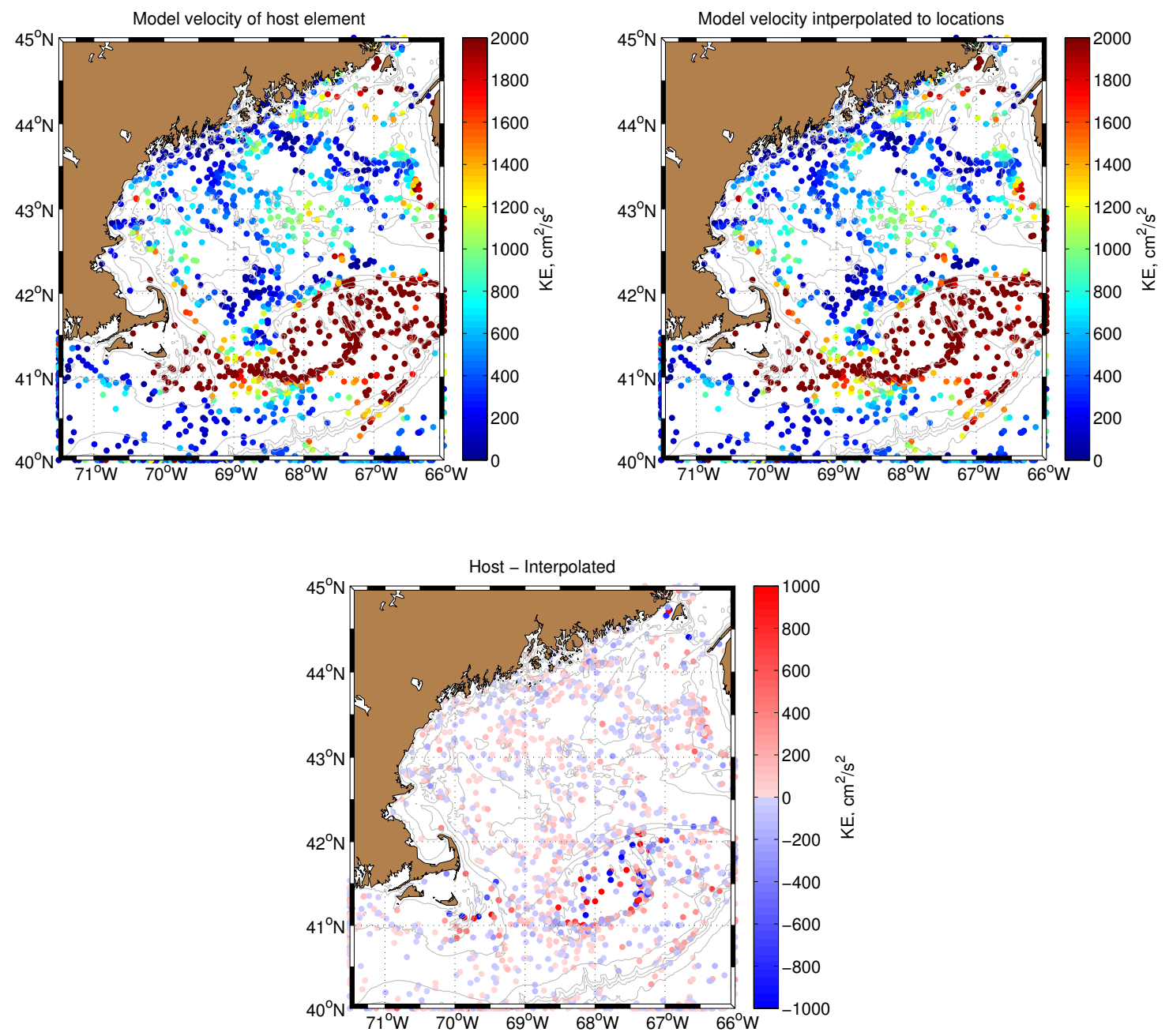

Figure 5-9: KE from model, host elements, interpolated to particle locations, and computed from 1st differences. Note that this figure depicts KE, not EKE. Lack of spatial coherence in KE indicates that particle tracking spatial interpolation does not introduce systematic bias.

interpolation, we only compute the kinetic energy, rather than the eddy kinetic energy. This is a direct measure of how well (or not) the interpolation compares to the original model output, and is not influenced by averaging (as EKE would be). 


\subsubsection{Testing the effect of low-pass filtering the drifter posi- tions}

During processing, we low-pass filter the drifter positions in order to concentrate the analysis on sub-tidal time scales. The magnitude of the tides in the Gulf of Maine is spatially variable, thus our attempt to filter the tides from the drifter positions could result in a bias in the resulting velocity. Does this low pass filtering introduce a bias?

To test this, we re-ran the tracking algorithm using the low-pass filtered model velocity fields, rather than the full velocity fields. These new fields we'll call the "low-pass filtered" trajectories to distinguish them from the model drifters (which are advected with the fully resolved tidal fields then processed to remove the tides). The release times and locations for the low-pass filtered drifters are identical to the release locations for the model trajectories, and the low-pass filtered drifters are processed identically to the model drifters except that the low-pass filtered drifters are not low-pass filtered with the PL66 filter since the advecting fields have already been smoothed before running the particle tracking algorithm. Finally, we compare the two fields to determine whether the process of low-pass filtering the drifter positions causes a bias.

The differences between the mean KE for the model and low-pass filtered drifters are shown in figure 5-10. An interesting pattern arises here: The model drifters are faster in the inflow region through the Northeast Channel and along the coastal current, but are slower along the northern flank of Georges Bank and along the western part of Nantucket Shoals. In the interior, also, the low-pass filtered drifters appear to mostly have more KE than the model drifters, indicating that some of the bias may be a result of the low-pass filtered procedure.

We have experimented with other low-pass filtering methods, including Fourier and boxcar filtering, but the results are mostly the same. Filters cannot remove all of the tidal energy, but can only hope to minimize it. This problem is complicated by the movement of the drifter through a spatially-varying tidal flow field. Perhaps a filtering 


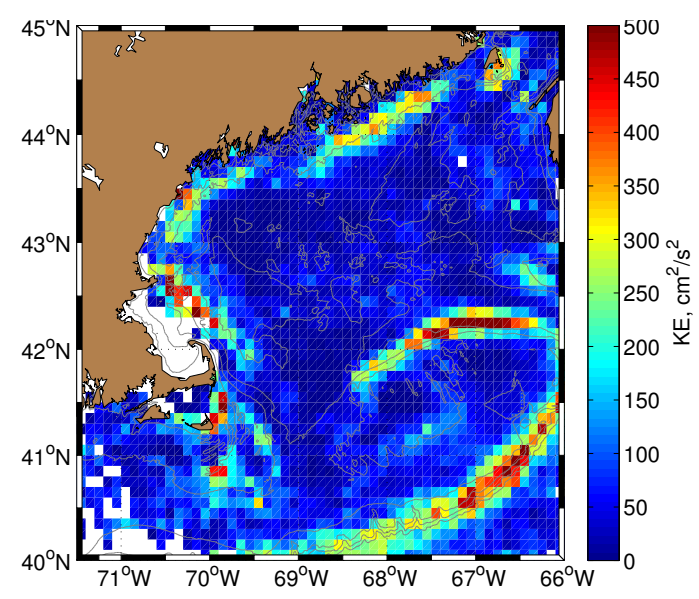

(a) Model KE

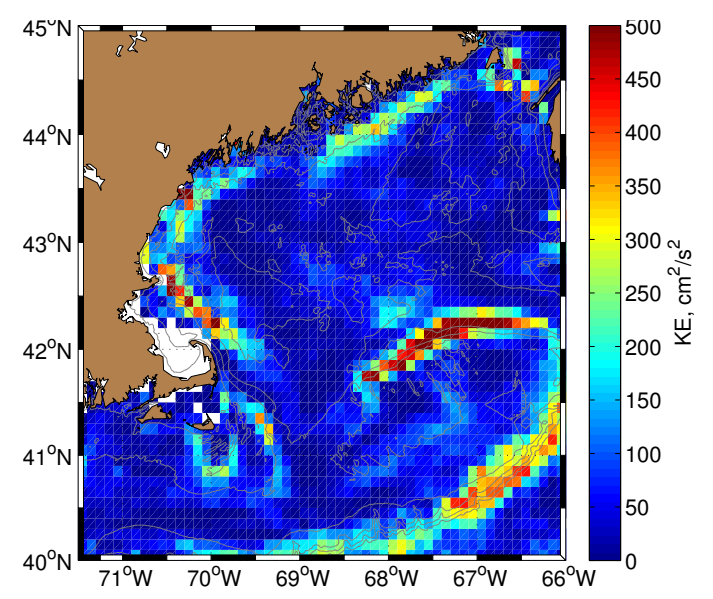

(b) Low-pass filtered KE

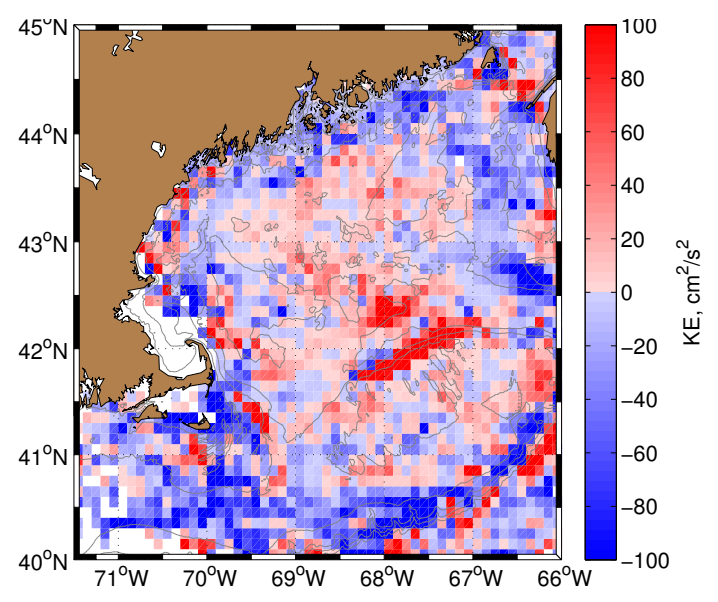

(c) Model KE - Low-pass filtered KE

Figure 5-10: KE from model drifters, low-pass filtered drifters, and difference.

scheme that takes this variability into account would reduce the bias introduced, however that is beyond the scope of this work.

\subsubsection{Testing the effect of computing the drifter velocity from 1st differences}

Another step in the processing is computing the drifter velocity by calculating changes in the drifter's position with respect to time. Here, we use the simplest approach: first differences, where the velocity is simply $\frac{\Delta x}{\Delta t}$. Does computing velocity from first 
differences introduce a systematic bias? Is there a higher order method that works better (e.g. spline fitting)? We compare the velocity derived from first-differences to the model velocity interpolated to the drifter locations in figure 5-11
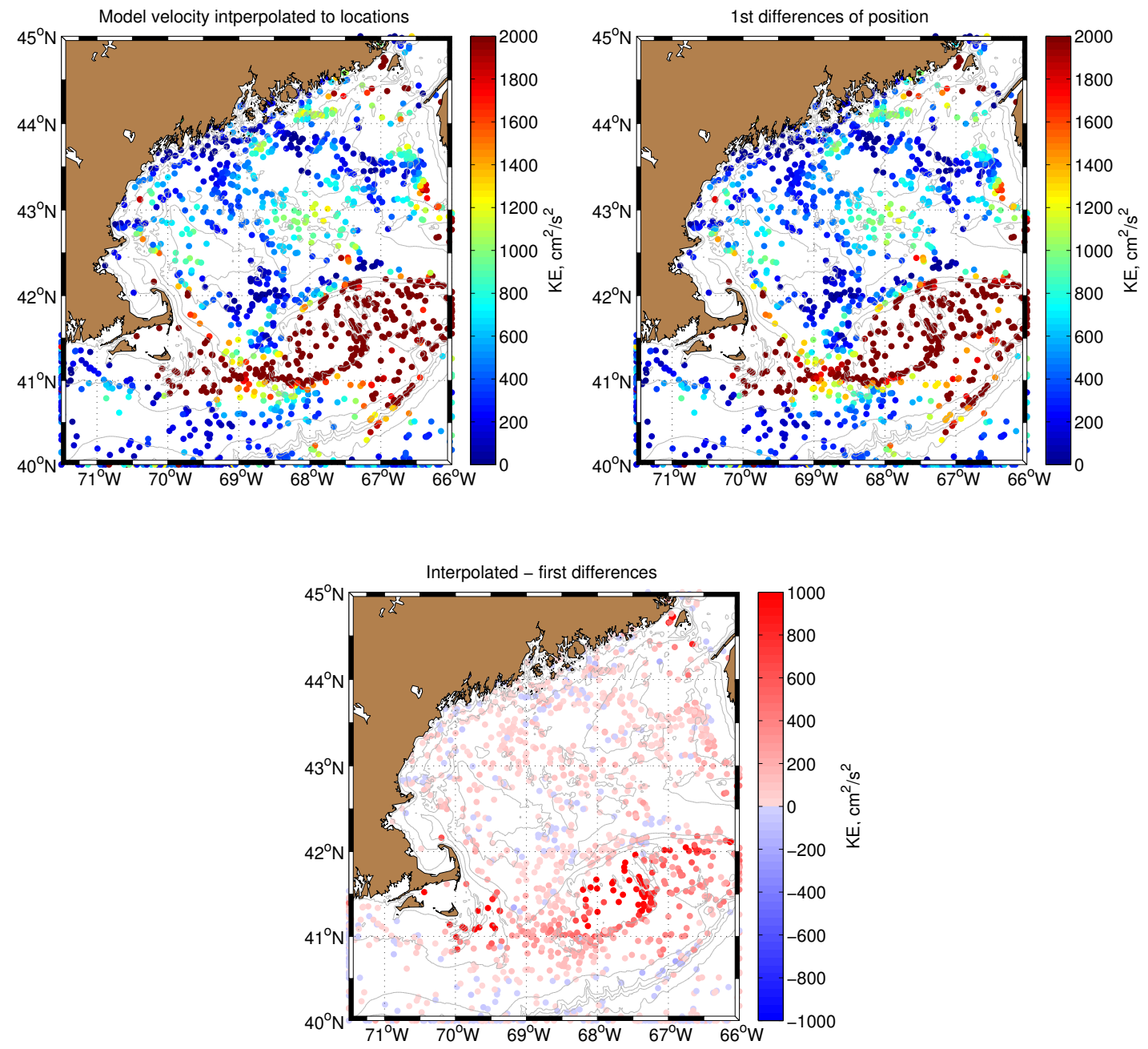

Figure 5-11: KE from model host elements interpolated to particle locations, computed from first differences, and the difference

Here, we see the most drastic bias yet: using first differences to compute the velocity from drifter positions systematically underestimates the KE of the flow, and that this effect is most drastic in regions of higher flow speed (namely, Georges Bank). Another method for computing the velocity from drifter positions is splines, which fit a curve to some number of position points. This method applied to this data, however, 
actually performs worse than first differences. Figure 5-12 shows histograms of the KE differences between first differences (red) and splines (blue). The mean difference is actually larger for the splines than for first differences.

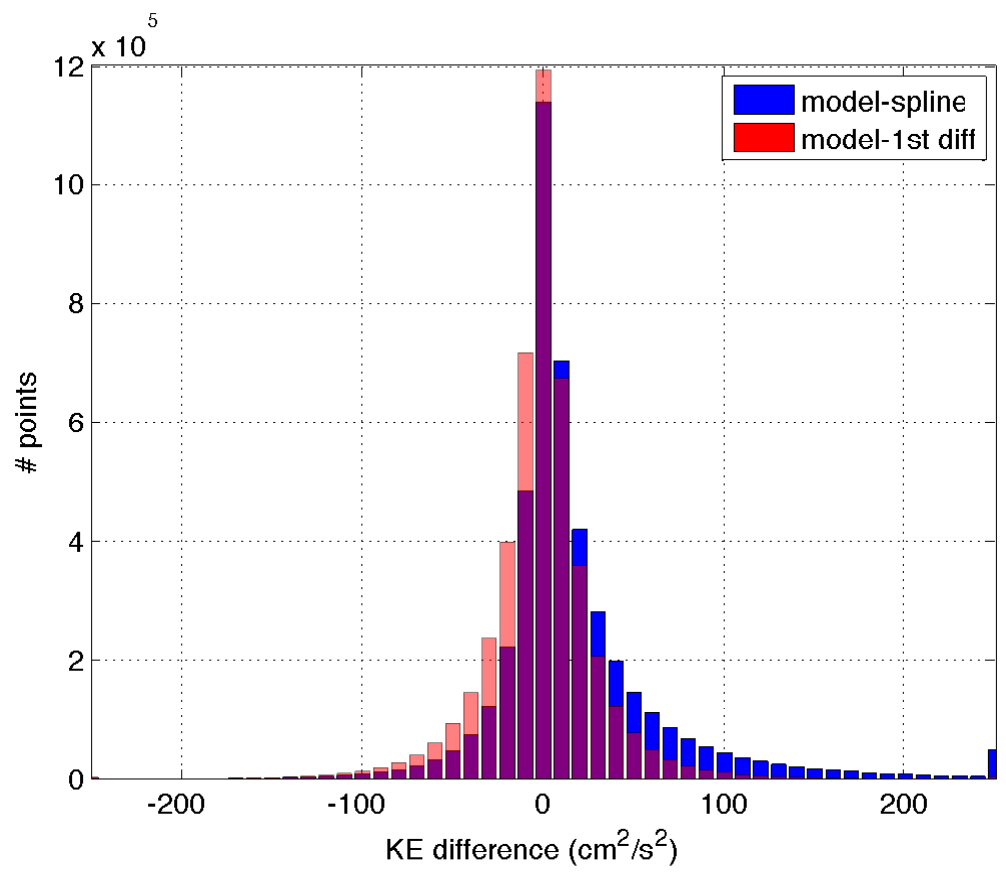

Figure 5-12: host-spline, mean $=29$, std $=121 ;$ host-1stdiff, mean $=9$, std $=123$

The main result here is that computing statistics about the flow from drifter positions results involves lots of assumptions and approximations that may result in biases, if not done carefully. Here, we were able to use a numerical model and numerical drifters to show that both low-pass filtering and computing velocity from the positions can lead to systematic error in the estimation of KE and EKE of the flow in the GOM. Because the errors are mostly small $(<20 \%)$, we will proceed with the processing done as outlined in section 4.3 (which are standard practices); further work is required to understand the optimal processing steps that should be taken for data in this region.

In general, the standard processing practices perform as well or better than the other steps tested in solving for velocity from drifter positions. Using first differences to compute velocity basically amounts to approximating the drifter path as a straight 
line between position fixes. The shorter the time between the position fixes, the better this approximation will be, and in the future drifters should report positions as frequently as possible.

\section{$5.6 \kappa$ derived from model drifters vs observations}

In the previous section, we evaluated different parts of the processing and calculations made with surface drifters; in this section we compare the values of $\kappa$ between the model drifters and observations computed using several different measures and averaged over different areas to see how well they compare. Again, we wish to use the model drifters to quantify uncertainty in the observations, and so we must first compare the two and attempt to understand any discrepancies.

\subsection{1 $\kappa$ over the entire GOM, model drifters}

Using the model drifters, we can compute the diffusivity for the entire GOM. As with the real data, we first compute the mean Lagrangian displacement:

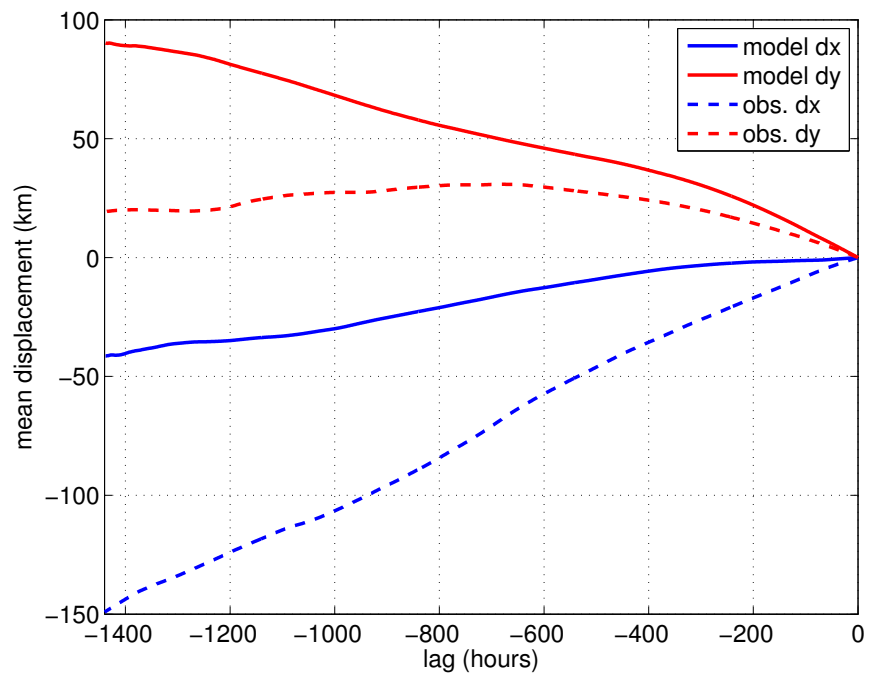

Figure 5-13: Mean Lagrangian displacements, model drifters

As with the observations, the meridional displacement is increasingly positive for 
larger negative lags, indicating the tendency of southward flow in the Gulf of Maine. The zonal displacement is increasingly negative for longer negative lags, which reflects the tendency of the drifters to move from west to east. The mean eastward and northward Lagrangian velocities are $(0.3,-3.7) \mathrm{cm} / \mathrm{s}$ respectively, consistent with the mean displacements. This behavior in the mean is expected due to the presence of the boundary, which will limit the northward and westward extent of drifter excursions. The meridional displacement is also larger than the zonal displacement. This is consistent with the fact that the coastline tends to be more aligned in the northsouth direction. The zonal model displacement is more negative than that of the observations, while the meridional model displacement is smaller than that of the observations, indicating that the model drifters have a little more tendency to move in the north-south direction than the observations, which tend to have more eastwest motion. This could be a result of the difference in release location of model and observational drifters and/or the poor offshore boundary condition of FVCOM. The former could result in observational drifters being preferentially deployed nearshore in the northern GOM (where the current flows more in the east-west direction), while the latter could result in drifters moving offshore across the shelfbreak south of the GOM where a more-realistic boundary flow would either move the drifters along the shelfbreak with the shelfbreak current.

Figure 5-14 shows the components of the diffusivity tensor for all model (solid lines) and observational (dashed lines) drifters computed from single particle statistics (with Eulerian means removed), and for drifter pairs. As in the case of the observations, the zonal component $\kappa_{x x}$ is slightly larger than the meridional component $\kappa_{y y}$ for lags less than about 12 days. Both the zonal and meridional components are larger for the model drifters than for the observations, which could be due to a slight mismatch in the spatial and temporal sampling between the model drifters and observations, or some interannual variability in the diffusivity, or some inconsistency between the model physics and those in the GOM, such as insufficient resolution of small scale processes and eddies, or an improper/over-idealized offshore boundary 


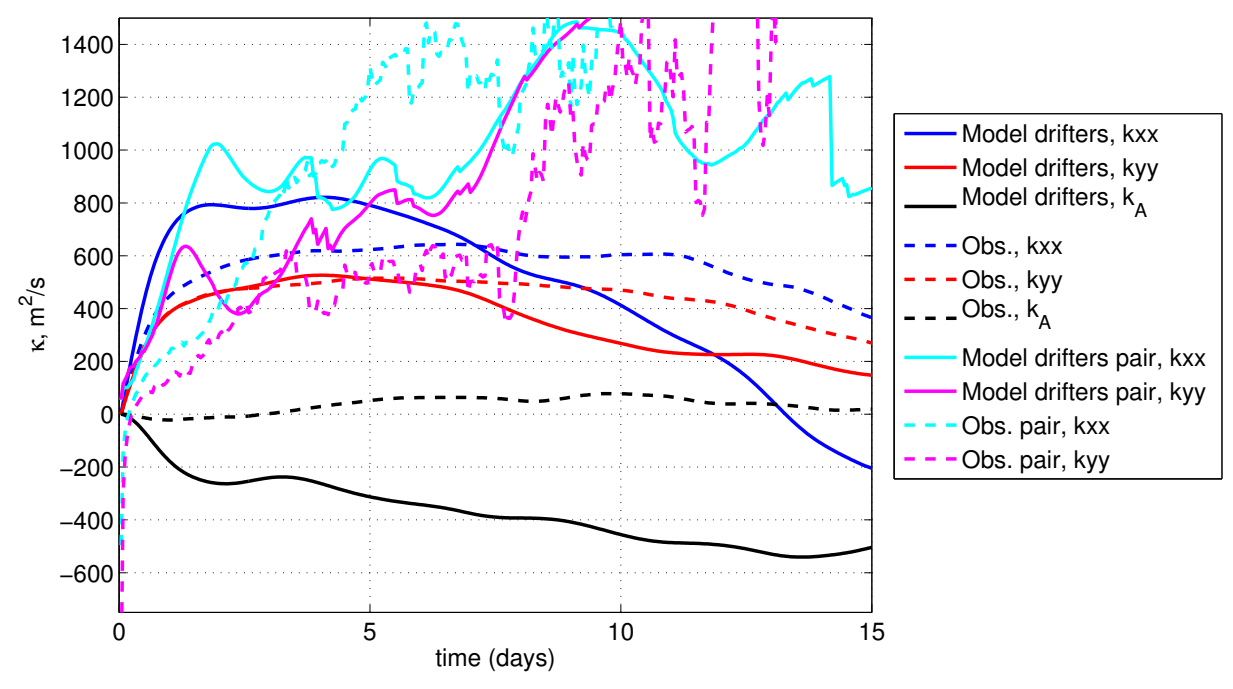

Figure 5-14: Diffusivity tensor for all of GOM

condition.

Additionally, we see very different patterns in the anti-symmetric part of the tensor. In the model, $\kappa_{A}$ is negative, reflecting the tendency for particles to veer in an anti-cyclonic sense. Again, there could be many reasons for this, but perhaps most likely is that the model drifters sample the area around Georges Bank more than the observational drifters do. The circulation around Georges Bank is strongly anticyclonic, so more data there will make the calculation for $\kappa_{A}$ increasingly negative.

\subsubsection{Pair dispersion and eddy diffusivity}

As with the observations, we can compute pair statistics for the model drifters. Here, due to the sheer number of drifters active at every time step, we have many more chance pairs than in the observations. Figure 5-15 shows the number of pairs as a function of time since "release".

Again, we compute the dispersion for all drifter pairs, and compute the pair diffusivity from equation 5.3 (figure 5-14). The number of drifter pairs in the model drifter dataset allows for much better statistics when computing the pair diffusivity, and thus no smoothing is applied as there was in the observations. As in the obser- 


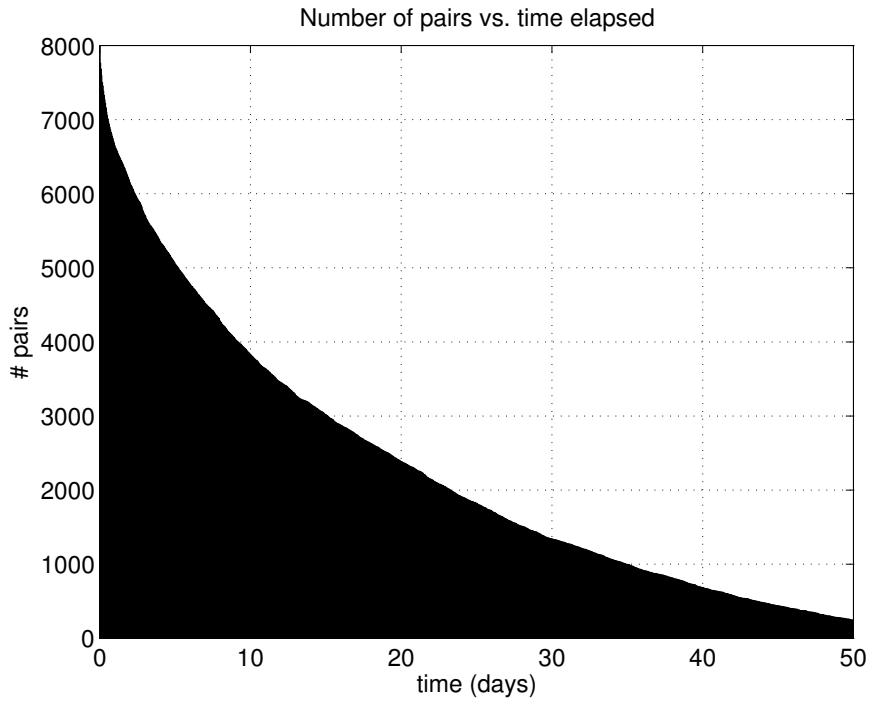

Figure 5-15: Number of drifter pairs as a function of time.

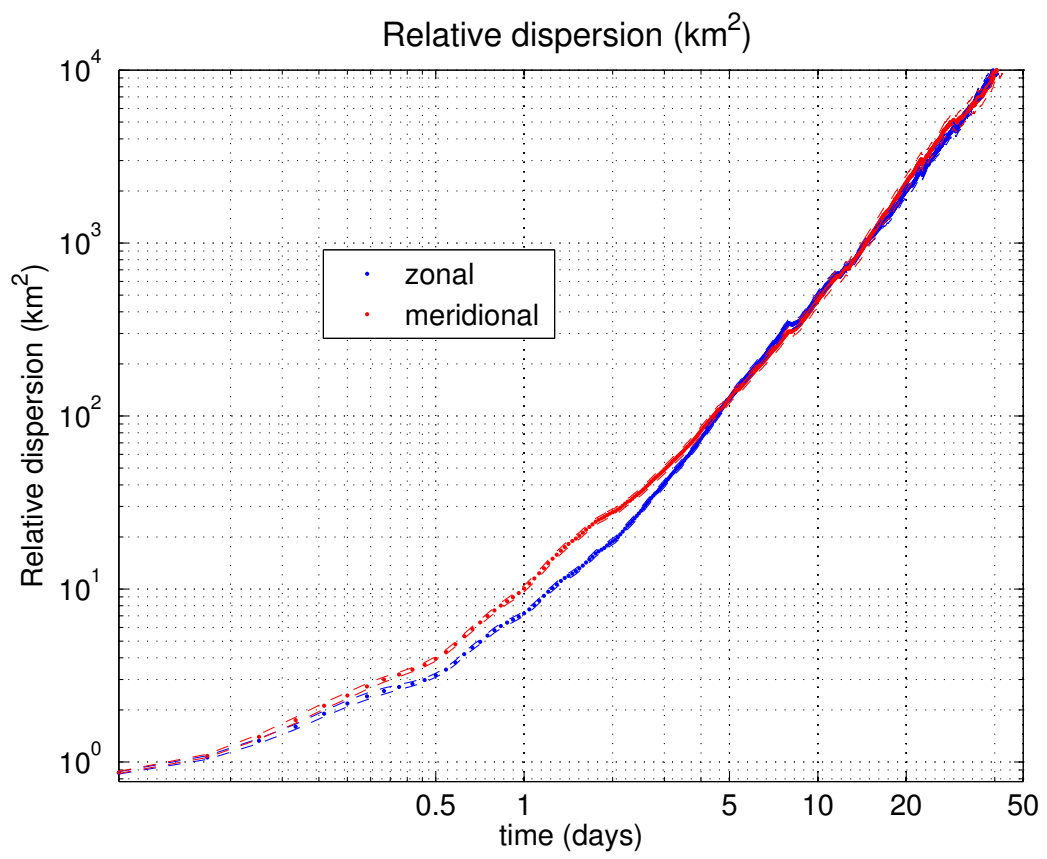

Figure 5-16: Relative dispersion as a function of time for all model drifter pairs in GOM data (dashed lines are standard error for each bin).

vations, the dispersion on scales less than 1-2 days (those less than the cutoff period for the PL66 filter) is relatively meaningless. In the observations, the intermediate period lasted from just over 3 days to between 10 and 20 days. Here, it appears that 
the relative dispersion continues to increase almost linearly (on the log scale) up until 50 days. Also, the dispersion and diffusivity are more isotropic $\left(\kappa_{x x}=\kappa_{y y}\right)$ in this period in the model drifters, whereas in the data the zonal component was larger.
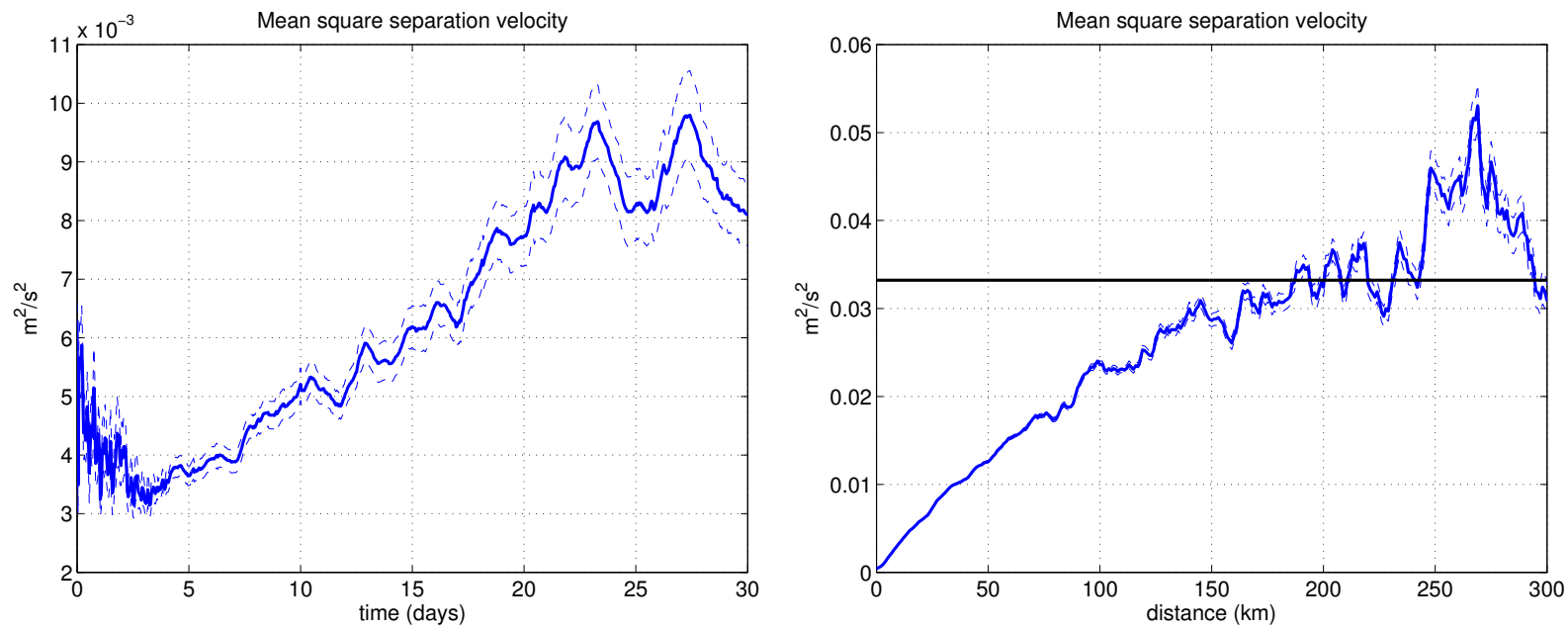

Figure 5-17: Separation velocity as a function of time (left) and separation distance (right)

Mean square separation velocity for the model drifter pairs (figure 5-17) shows slightly different patterns than the observations. The separation velocity does not asymptote until after 20 days in the model, as compared to the observations (more like 10 days), and even then it is much lower than what is to be expected of two random particles (close to $.03 \mathrm{~m}^{2} / \mathrm{s}^{2}$ ). The horizontal scale is also larger in the model (150km vs. $50-100 \mathrm{~km}$ in the observations). There are two possible explanations for this: either the model scales are larger than the real world, or the 80 observational drifter pairs are simply not enough to effectively resolve the space and time scales. These explanations will be explored in the bootstrapping section.

\subsubsection{Three sub-regions: Interior GOM, Coastal Current, and Georges Bank}

Figure 5-19 shows the diffusivity tensor computed from model drifter and observations in the three geographic bins shown (observations reproduced from previous chapter), 
with the addition of the bin-specific estimate of $\kappa_{\text {pair }}$, discussed below. Focus first on the single-particle estimate with the trajectory mean removed (solid lines).The pattern is similar to that obtained in the data: Highest values of $\kappa$ are found in the $\mathrm{CC}$ and $\mathrm{GB}$ bins, lowest in the interior GOM, and the antisymmetric components are all consistent with the tendency for cyclonic or anticyclonic flow in the respective bins.

The patterns are similar for the single-particle diffusivity with Eulerian mean removed. This is in contrast to the observations, where the $\kappa_{E u l}$ had slightly different patterns. Most notably, the diffusivity is more isotropic in the GB bin than in CC (in the observations, the $\mathrm{CC}$ bin was more isotropic than GB).

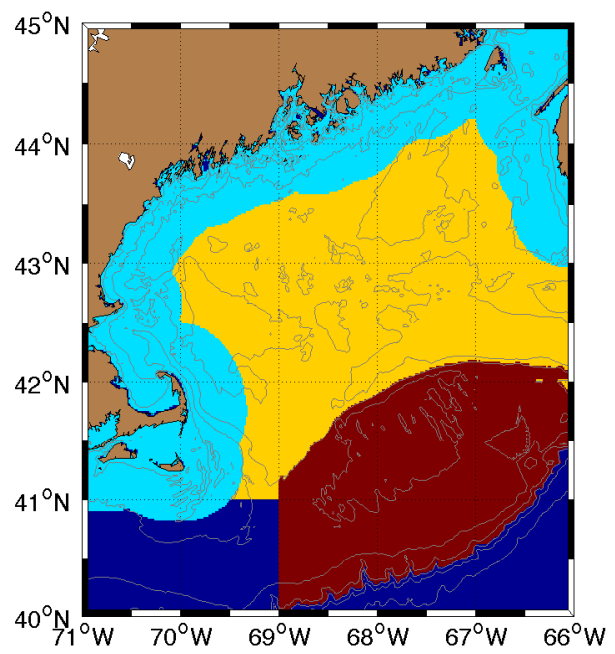

Figure 5-18: Three bins for regional diffusivity estimates. Light blue is CC bin, yellow is GOM bin, red is GB bin.

With the large number of model drifter pairs, it is now possible to compute pair dispersion and diffusivity in the geographic bins pictured in figure 5-19. For each drifter pair, we look at the location of the pair at the "release" time and label each pairs initial bin. We then compute the average dispersion and diffusivity for all pairs originating from the same bin. There are almost twice as many pairs in the $\mathrm{CC}$ bin as in the other bins (figure 5-20), but the length of these pairs tends to be shorter than the other bins, such that after 15 days, there are more pairs in both the GOM 

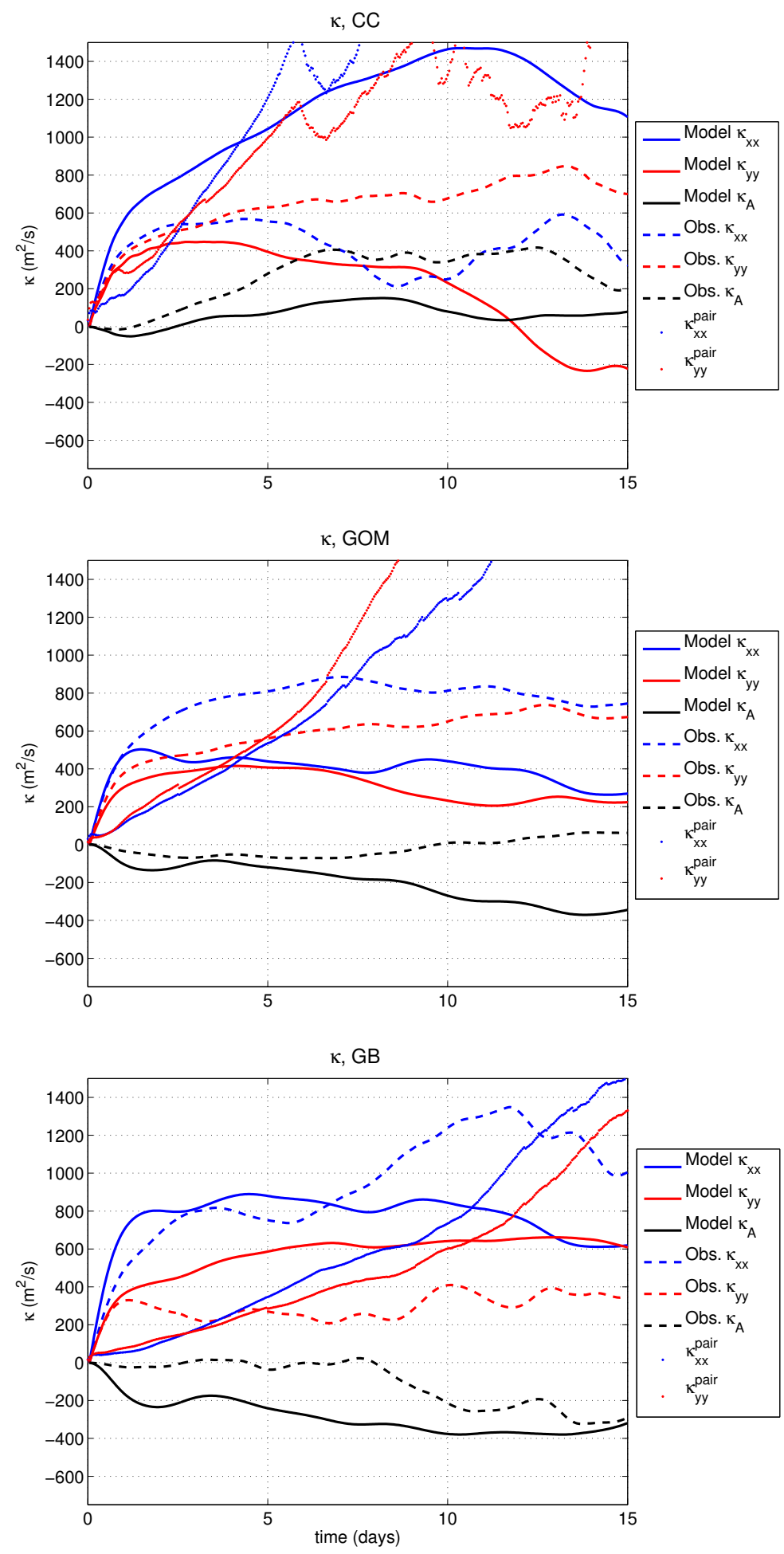

Figure 5-19: Diffusivity in three bins in figure 5-18 (same as previous chapter). Dashed lines are observations, solid lines are model drifters, dots are pair diffusivities from model drifters. $\mathrm{x}$-axis is lag in days. 
and GB bins.

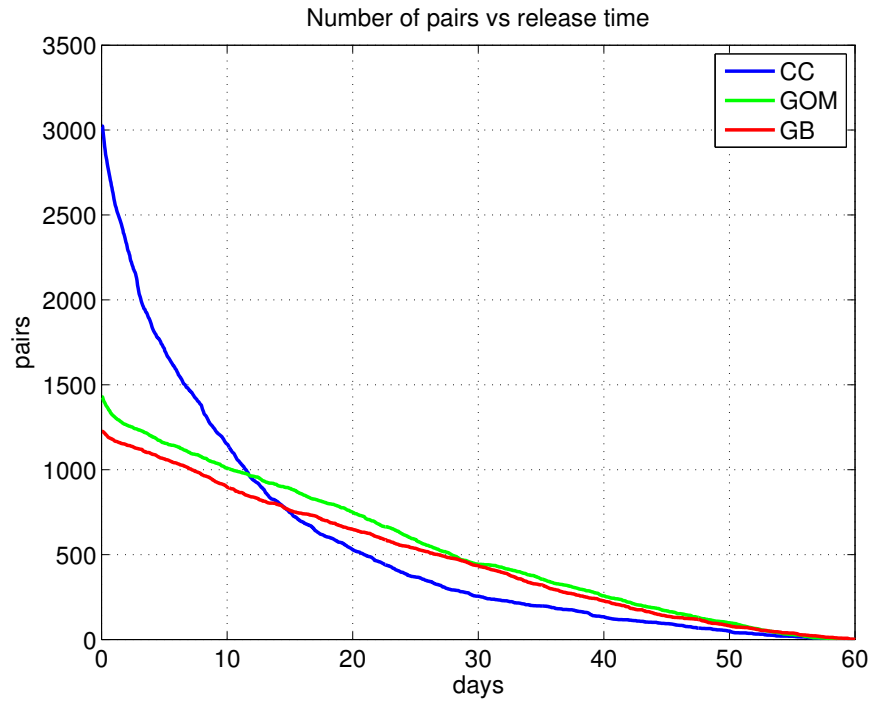

Figure 5-20: Number of model drifter pairs as a function of time for each of the three bins.

Figures 5-21 and 5-19 show the dispersion and diffusivity, respectively, as a function of release time for each bin. As in the single particle diffusivities, the largest values for $\kappa_{\text {pair }}$ at lags less than 8 days are found in the $\mathrm{CC}$ bin. The three-regime pattern in the pair diffusivity is most notable in the CC bin, but is also present in the GOM bin. It is difficult to tell whether the same pattern is occurring in the GB bin. Also interesting to note: the pair diffusivity is actually lowest in the GB bin rather than the interior GOM bin, which could be due to strongly coherent tidal motion not actually causing two particles to separate, but rather be simply sloshed back and forth.

To check the spatial and temporal scales associated with the drifter pair separation, we again compute the separation velocity as a function of time and separation distance (figure 5-22). The pair velocity asymptotes to the uncorrelated value much faster in the CC bin than in either of the other two bins. For all times less than 15 days, the separation velocity is much higher in the $\mathrm{CC}$ bin, which implies that a patch there will last for a shorter amount of time than in either of the other bins. Also of note, the separation velocity at spatial scales less that $50 \mathrm{~km}$ is larger in the 

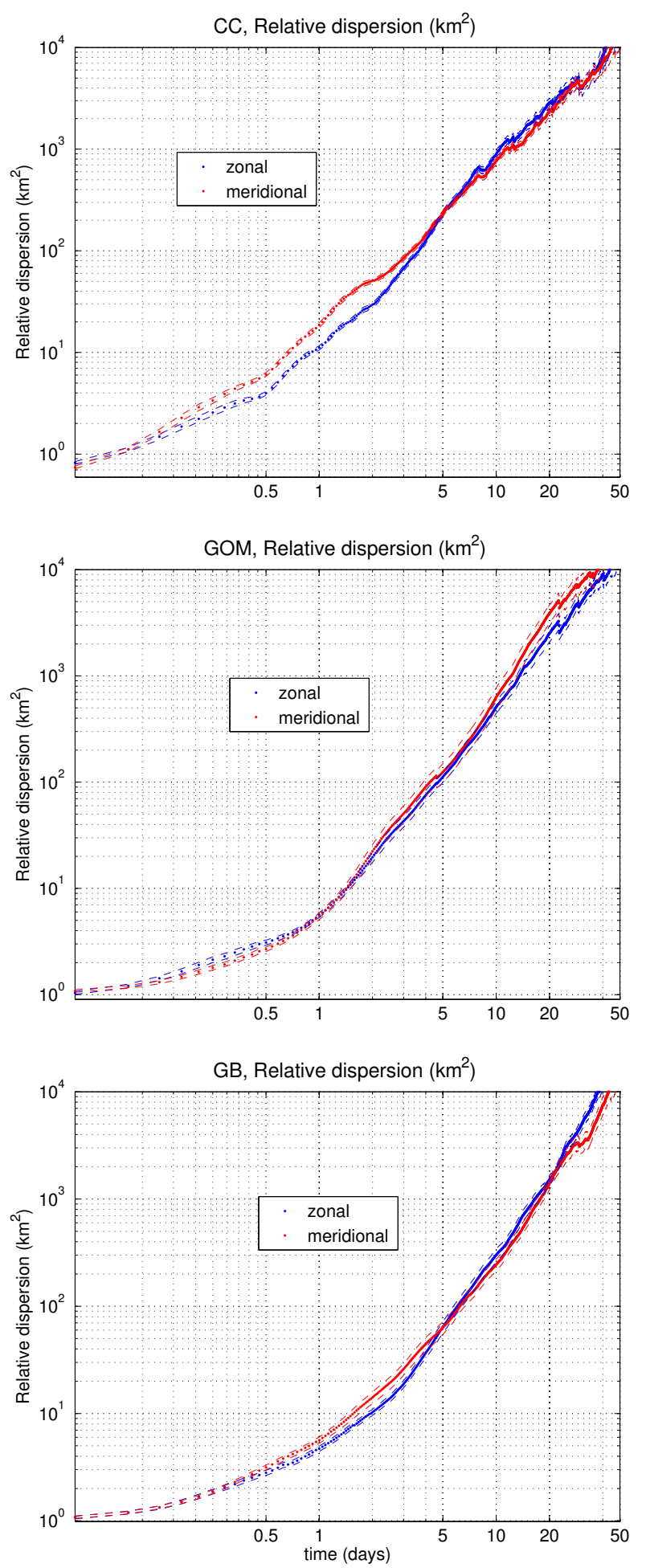

Figure 5-21: Model drifter pair dispersion in 3 sub-regions. $\mathrm{x}$-axis is lag in days. 
$\mathrm{CC}$ bin. Between 50 and $100 \mathrm{~km}$, the three bins appear identical, and all asymptote to the single-particle value at scales larger that $100 \mathrm{~km}$. These plots imply that in the $\mathrm{CC}$ bin there is are motions on short space and time scales that would cause a patch of tracer to spread that are not found in the rest of the Gulf of Maine.
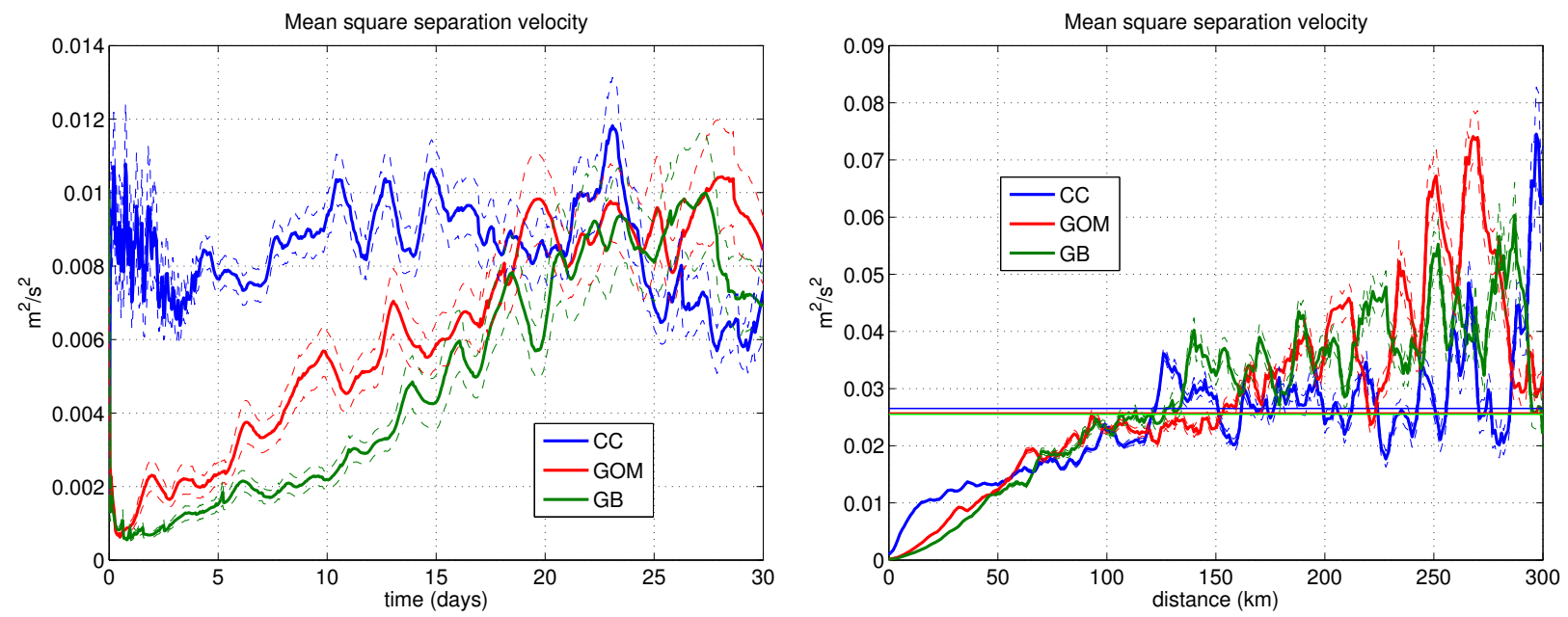

Figure 5-22: Separation velocity vs time (left) and separation distance (right)

\subsubsection{Diffusivity from Green's Functions}

It was suggested above that since $\kappa_{E u l}$ agreed more favorably with $\kappa_{\text {pair }}$ than $\kappa_{t r a j}$, that $\kappa_{E u l}$ was the more appropriate measurement. We can verify this by computing $\kappa$ in an independent manner, using Green's functions as described in section 4.3

Using the same FVCOM model output, we computed $\kappa$ via the Green's function method and compared it to the other methods described above (figure 5-23). We can see good agreement between the Green's function approach and the single-particle diffusivity with Eulerian mean removed, except in the case of $\kappa_{A}$. The magnitude of $\kappa^{\text {traj. }}$ is much larger than any of the other methods. The maximum amplitude of $\kappa^{\text {pair }}$ is of comparable magnitude to $\kappa^{E u l}$ and $\kappa^{G F}$, however the pair diffusivity increases to this value over a much longer time than any of the single-particle methods. 


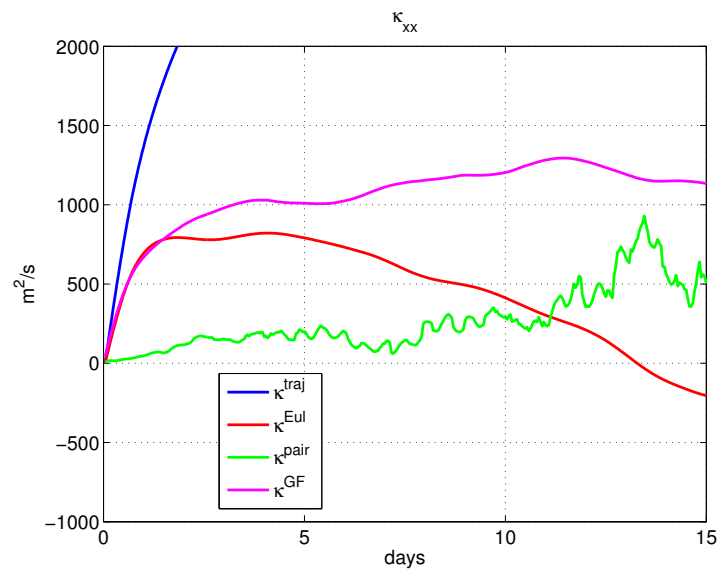

(a) $\kappa_{x x}$

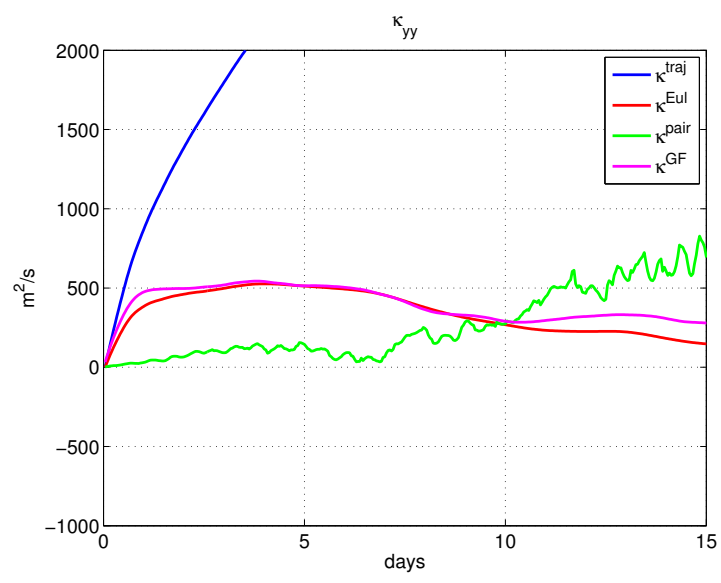

(b) $\kappa_{y y}$

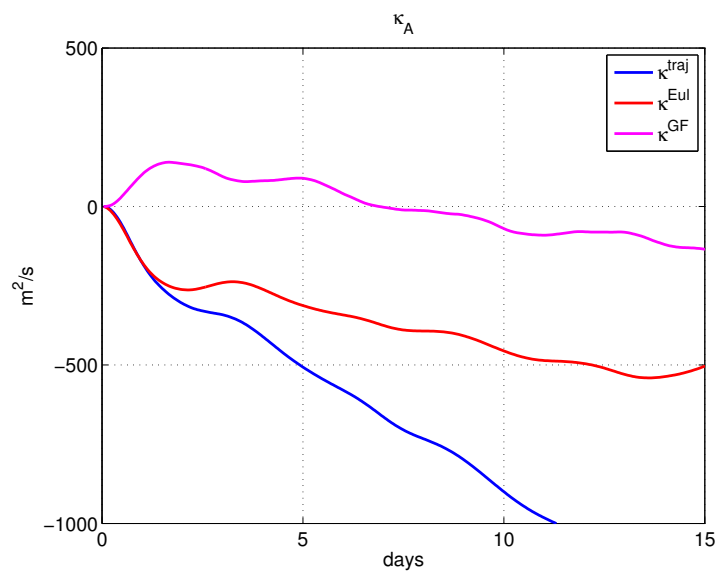

(c) $\kappa_{A}$

Figure 5-23: Diffusivity comparing different methods, all GOM.

\subsection{Diffusivity uncertainty estimates}

One of the goals of this work is to gain some understanding of the uncertainty of estimating diffusivity from surface drifters. We now compute diffusivity from subsets of the drifter data to calculate bootstrap uncertainty estimates, as described above. For the single-particle estimates with Eulerian mean removed, we use subsets of 50, 150, 400, and 1000 drifters repeated 100 times, while for the pair estimates we use subsets of 50, 150, 450, and 1000 pairs repeated 100 times. It is important to keep in mind that in the observations, we have approximately 400 drifters and 80 drifter 
pairs. A similar analysis has been performed on $\kappa_{t r a j}$, however, we only show that of $\kappa_{E u l}$ because it is likely the better estimate (see discussion above).

\subsubsection{Single-particle, all GOM}

Single-particle error estimates for $\kappa_{E u l}$ over the entire GOM are shown in figure 5-24. Depending on the goal of the analysis, this figure can be interpreted in many ways. If the goal is to obtain an estimate for $\kappa$ that is different from zero, or to know the order of magnitude, a small number of drifters suffices (approximately 150 drifters would work for lags less than 8 days). If one wishes to discern differences between zonal and meridional values, however, a larger number is required. Indeed, 1000 drifters (twice as many as are currently available in the GOM) are barely enough for this goal. The estimate using 400 drifters, close to that currently available, shows that $\kappa$ is known

to within approximately $\pm 500 \frac{m^{2}}{s}$ at a lag of 8 days. For further comparisons, we will use the uncertainty at 8 days.

\subsubsection{Single-particle, three sub-regions}

Above, we diagnosed the diffusivity in three sub-regions, but with the available number of drifters, is it possible to infer the differences between the bins? When smaller numbers of drifters are used, the errors (plotted as $\pm 2 \sigma$ ) overlap, indicating that, to 95\% confidence, we cannot discern between the diffusivity estimates. If, however, the number of drifters is increased to 400, our estimates are more precise, and the differences in $\kappa$ between the different bins now can be evaluated with more confidence. The difference is even more pronounced using 1000 drifters.

Interestingly, we notice from these figures (figures 5-25, 5-26, 5-27) that the uncertainty in the estimate of $\kappa$ is much larger in the $\mathrm{CC}$ bin than either of the other two bins, almost twice as large as that of the GOM or GB bins. It is unclear from this analysis exactly why this may be the case, however there are some possibilities. There are some variable components to the flow that are unique to the $\mathrm{CC}$ bin, e.g. 

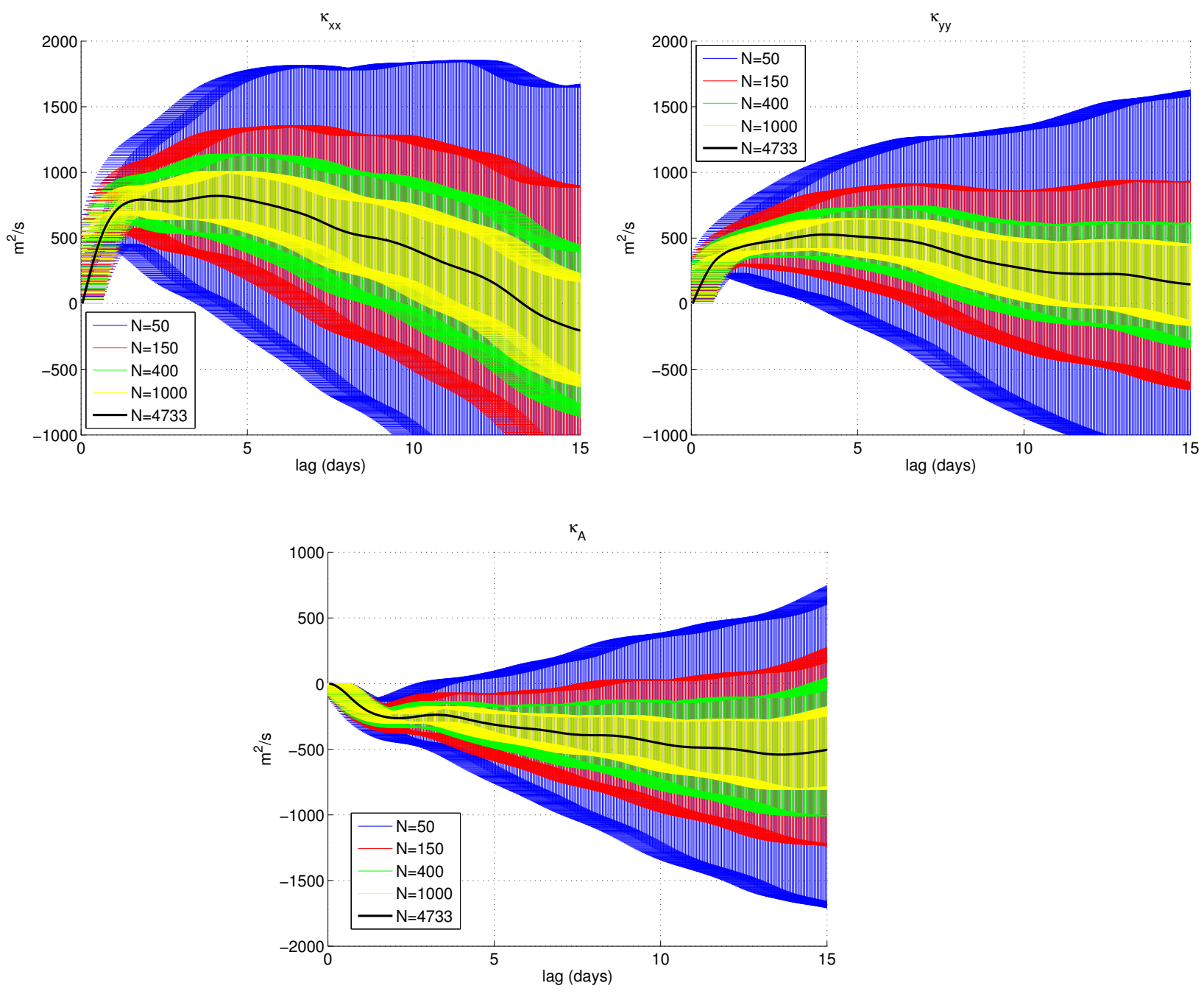

Figure 5-24: Diffusivity error (for all of GOM) estimated by bootstrapping with 100 realizations with 50, 150, 400, and 1000 drifters. Error bars are \pm 2 standard deviations.

wind-driven upwelling and downwelling, buoyant river discharges, etc., which cause variability in that bin but not elsewhere. It could be that these transient motions are the reason for the increased uncertainty in the $\mathrm{CC}$ bin, however further work is required to test this hypothesis.

Figure 5-28 shows the diffusivity at a lag of 8 days by component with uncertainty estimates for 50, 150, 400, and 1000 drifters. The uncertainty is so large for the CC bin that it cannot be distinguished from the other two bins, even for 1000 drifters. For the other two bins, however, the uncertainties are smaller, which allows for a 

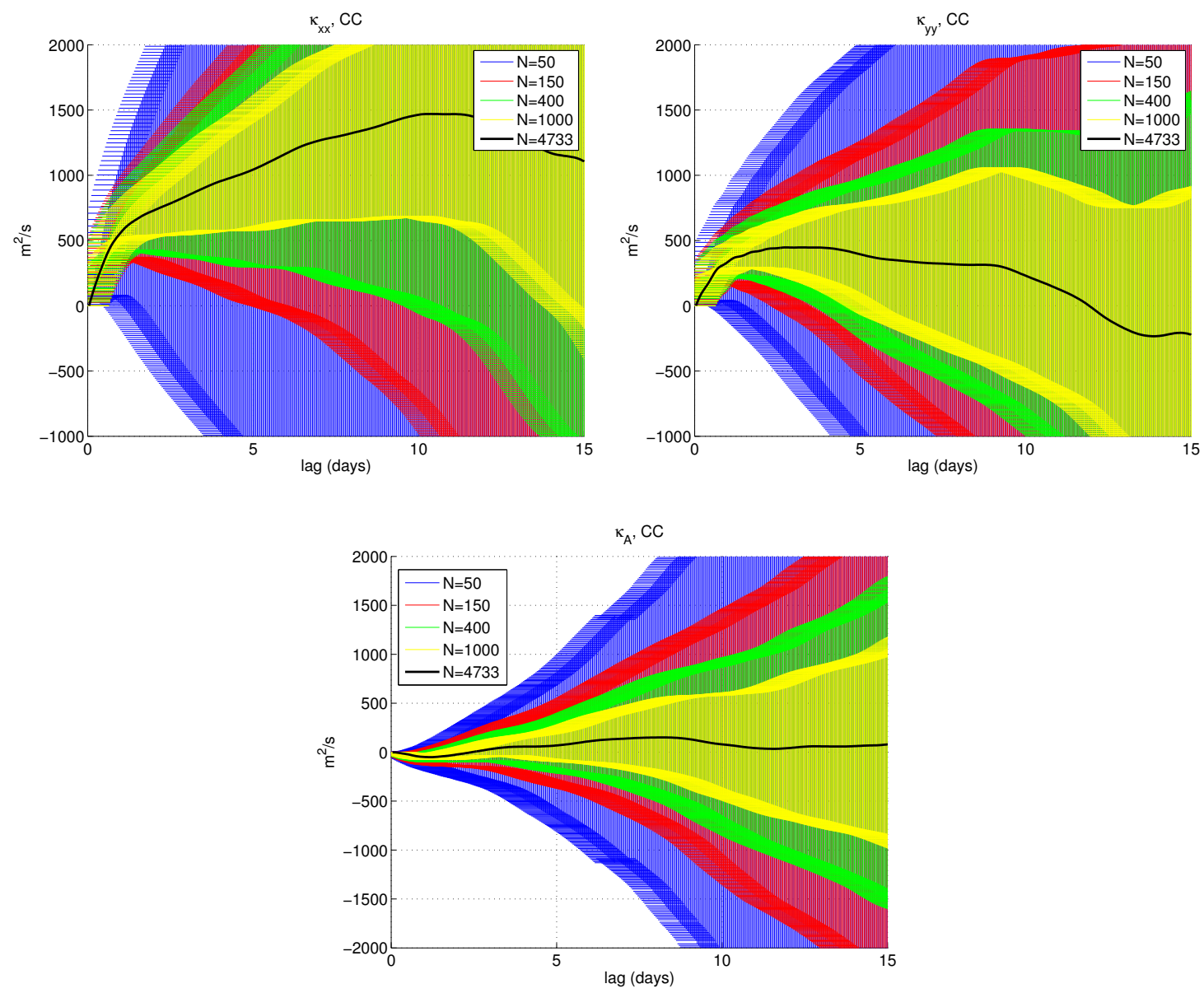

Figure 5-25: Diffusivity errors (for CC subregion) estimated by bootstrapping in bins in figure 5-19

more confident assessment of the patterns of diffusivity. This indicates that indeed the diffusivity in GB is larger than that in GOM. Meanwhile figure 5-29 shows the uncertainty estimates for the three components of $\kappa$ for each bin for 400 drifters for lags up to 15 days. Again, the CC bin is too uncertain with 400 drifters to discern from zero, much less the other bins, but it appears that 400 drifters is the lower limit for discerning between the GB and GOM bins. 

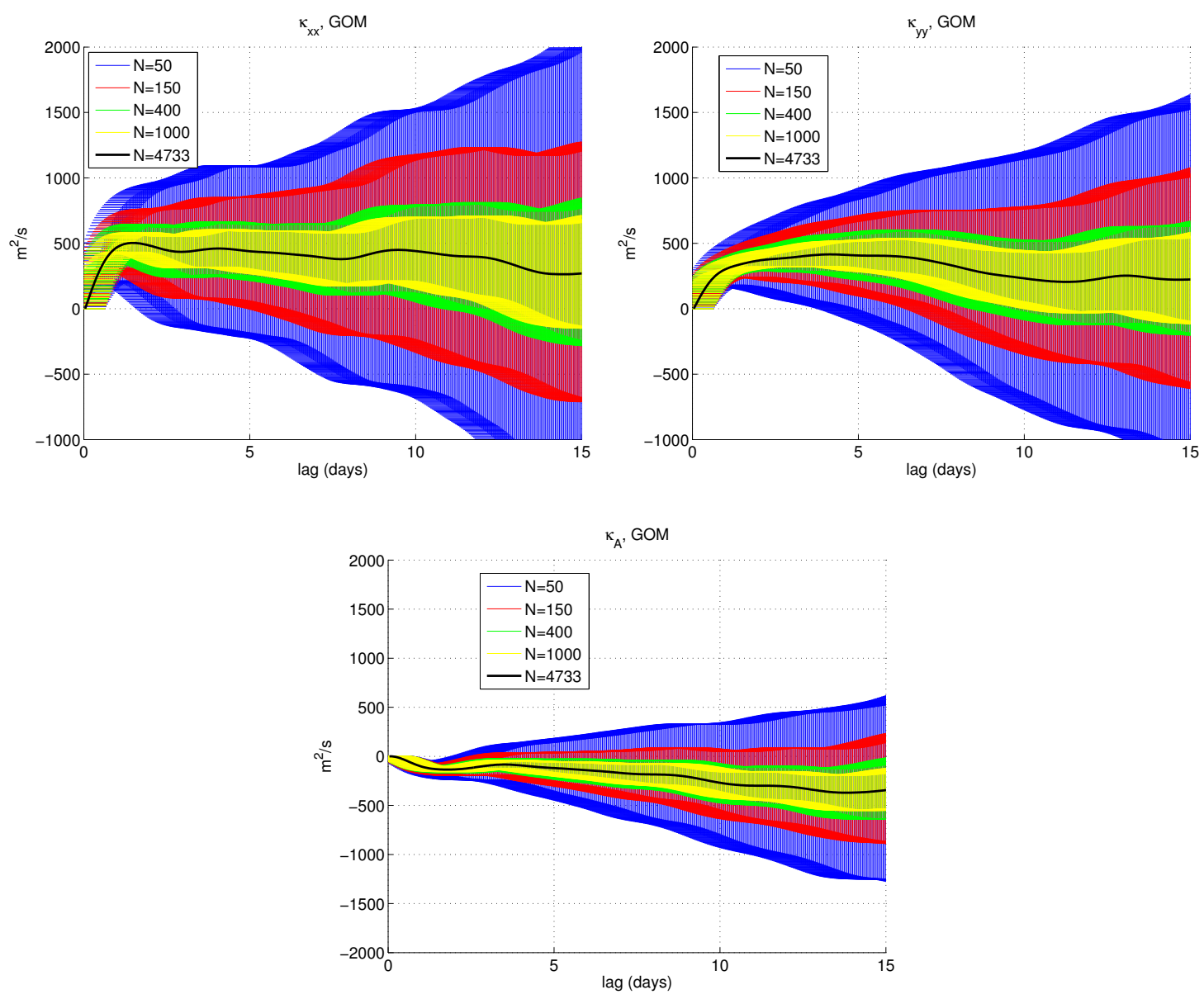

Figure 5-26: Diffusivity errors (for GOM subregion) estimated by bootstrapping in bins in figure 5-19 

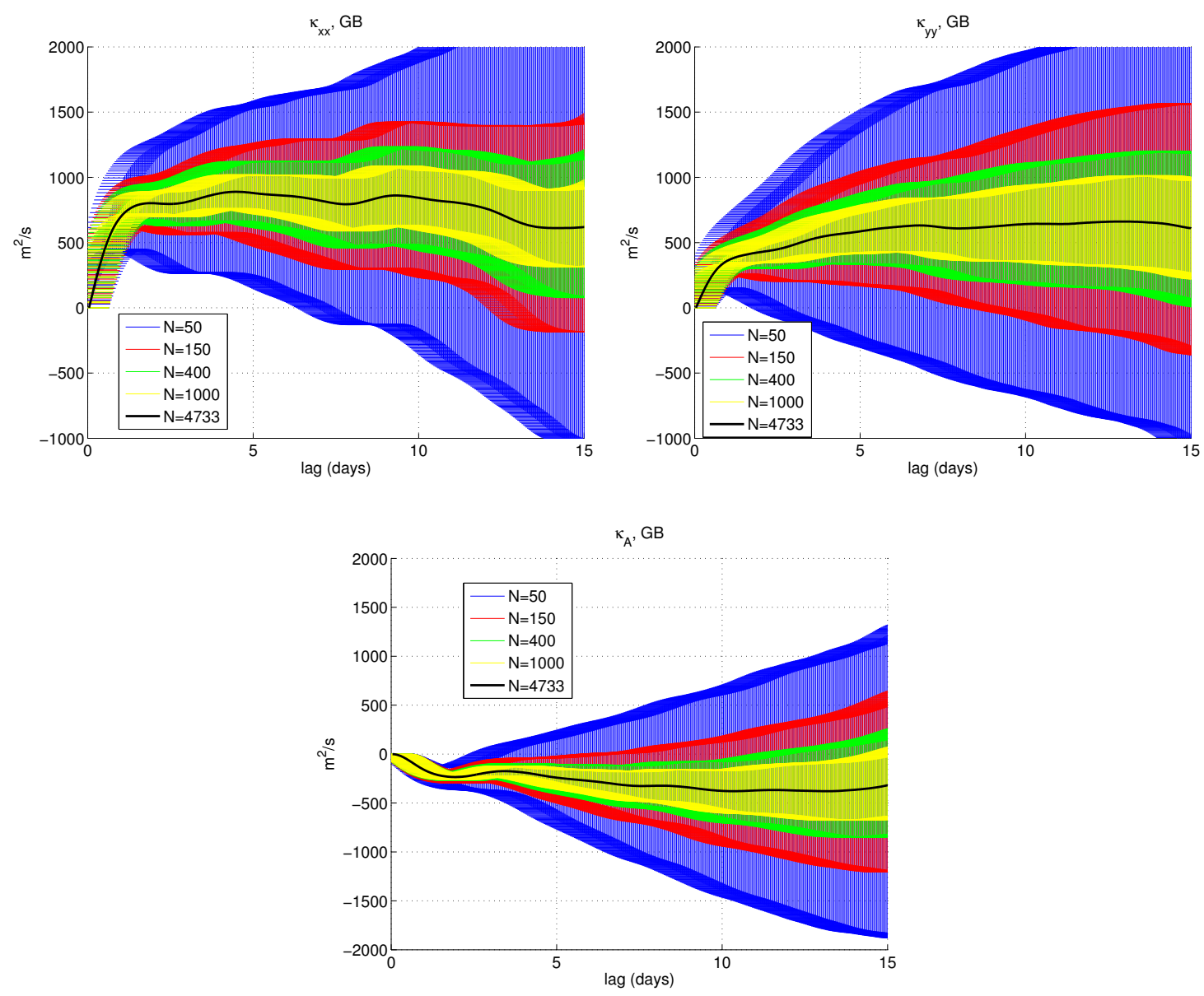

Figure 5-27: Diffusivity errors (for GB subregion) estimated by bootstrapping in bins in figure 5-19 

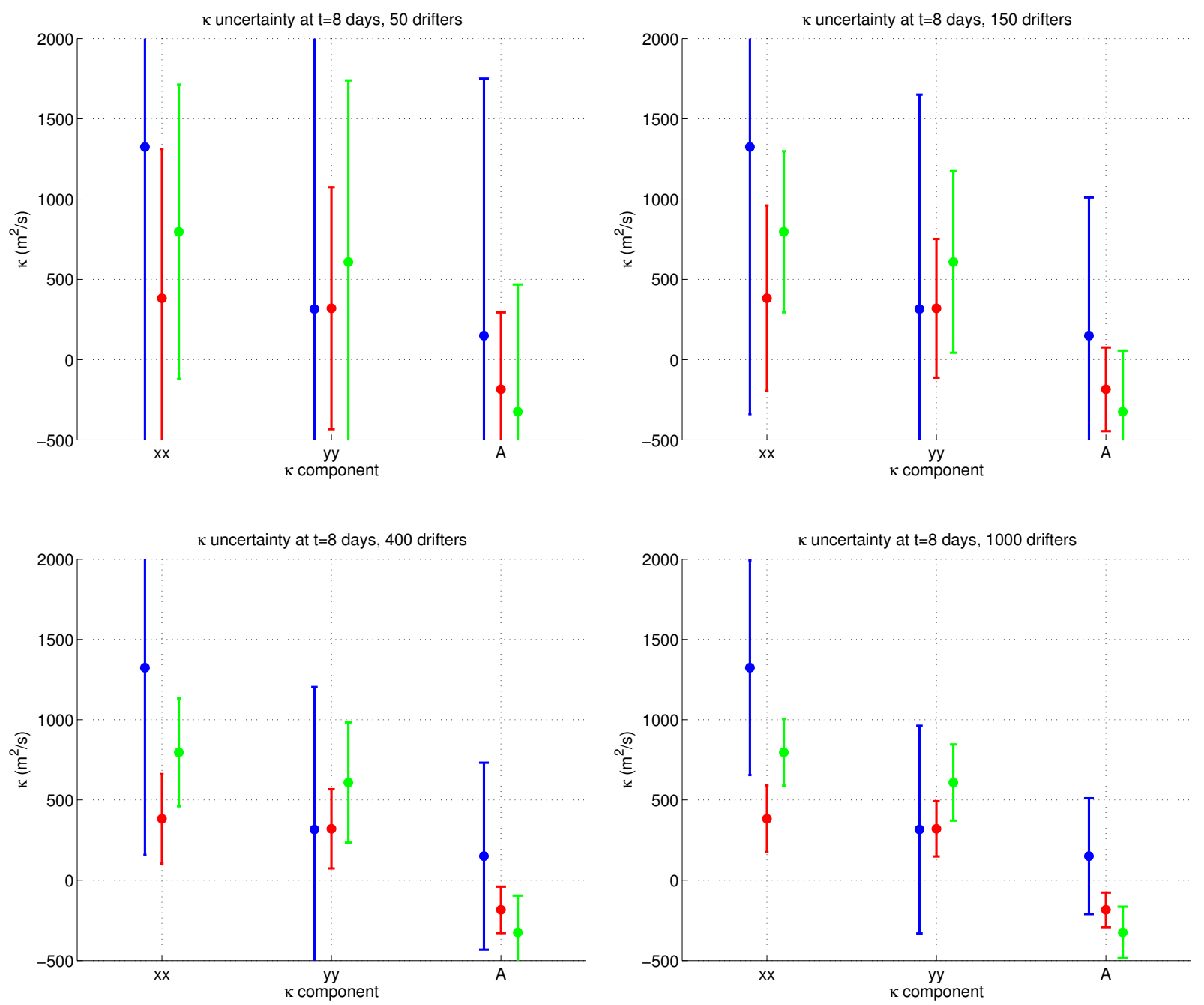

Figure 5-28: Diffusivity errors estimated at 8 day lags by component and bin, CC (blue), GOM (red), and GB (green). 

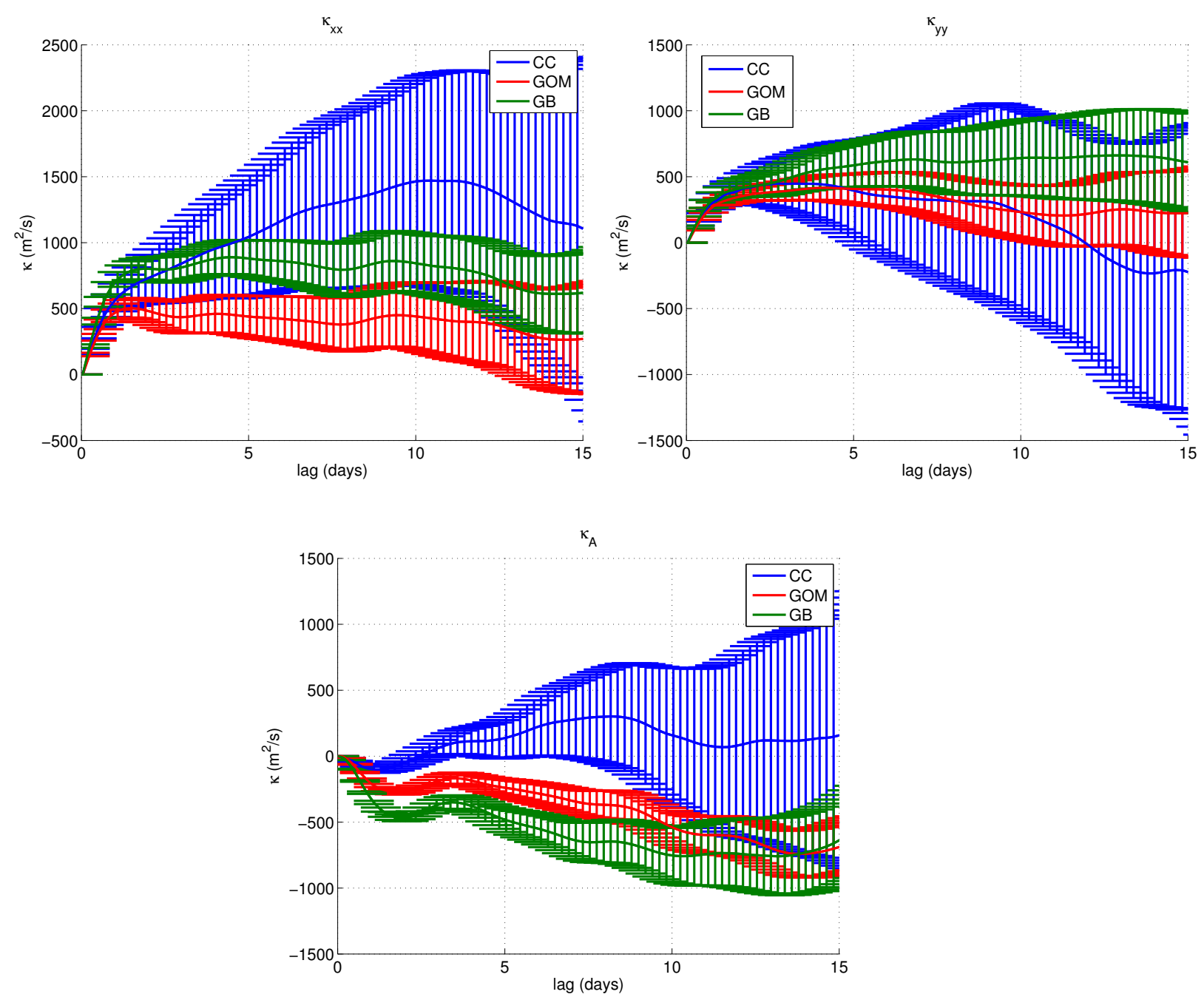

Figure 5-29: Diffusivity errors estimated by bootstrapping with 400 subsamples. 


\subsubsection{Particle pairs}

By taking subsets of different numbers of drifter pairs, we can get bootstrap estimates of errors on pair dispersion, diffusivity, and separation velocity. The errors using pair statistics tend to be larger than that of the single-particle statistics, such that 1000 pairs are needed simply to discern the diffusivity from zero (5-31). There is still merit in the pair dispersion estimates, however; more interpretation of this is found in the discussion section
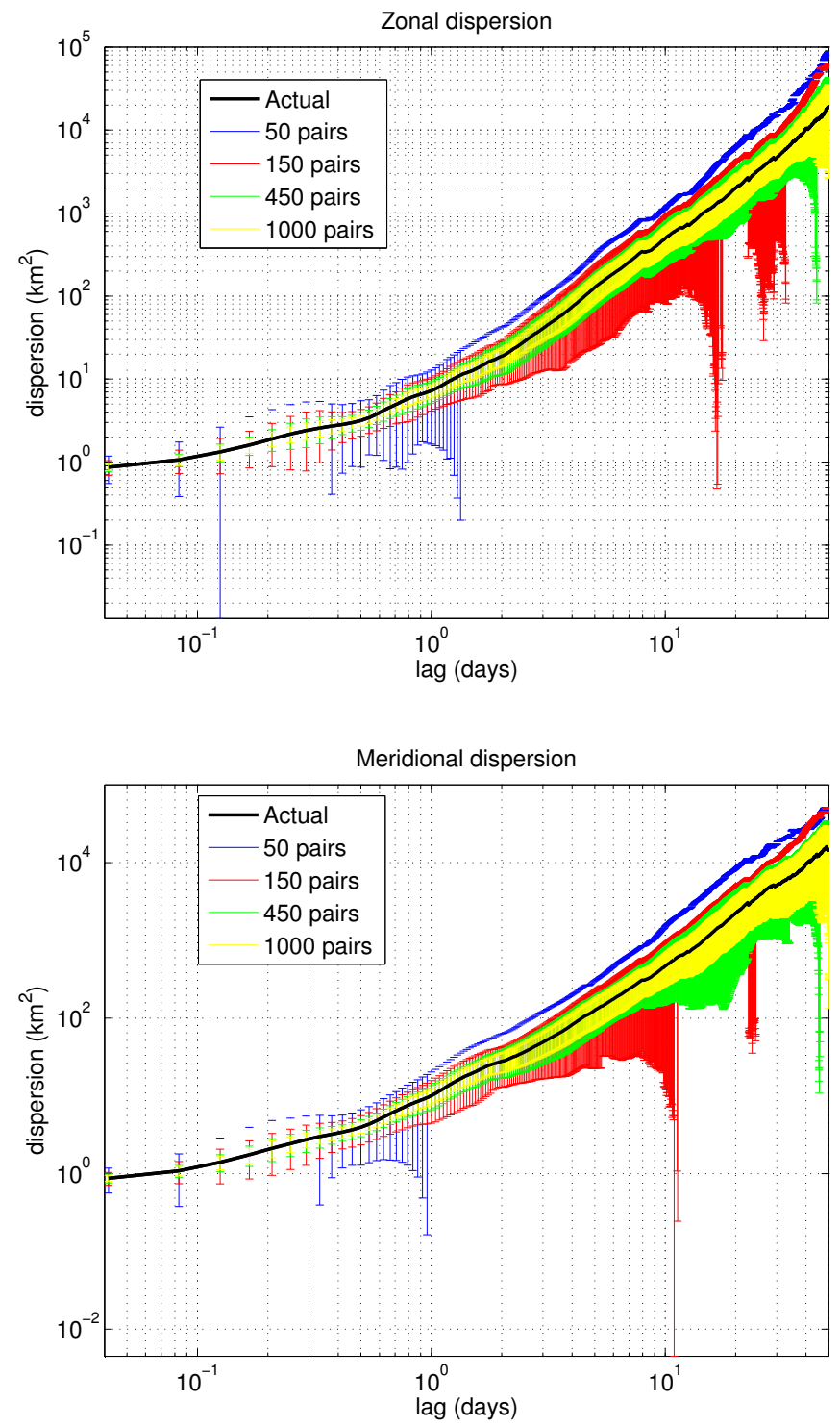

Figure 5-30: Errors on dispersion for pair statistics. Time (x-axis) is in hours. 

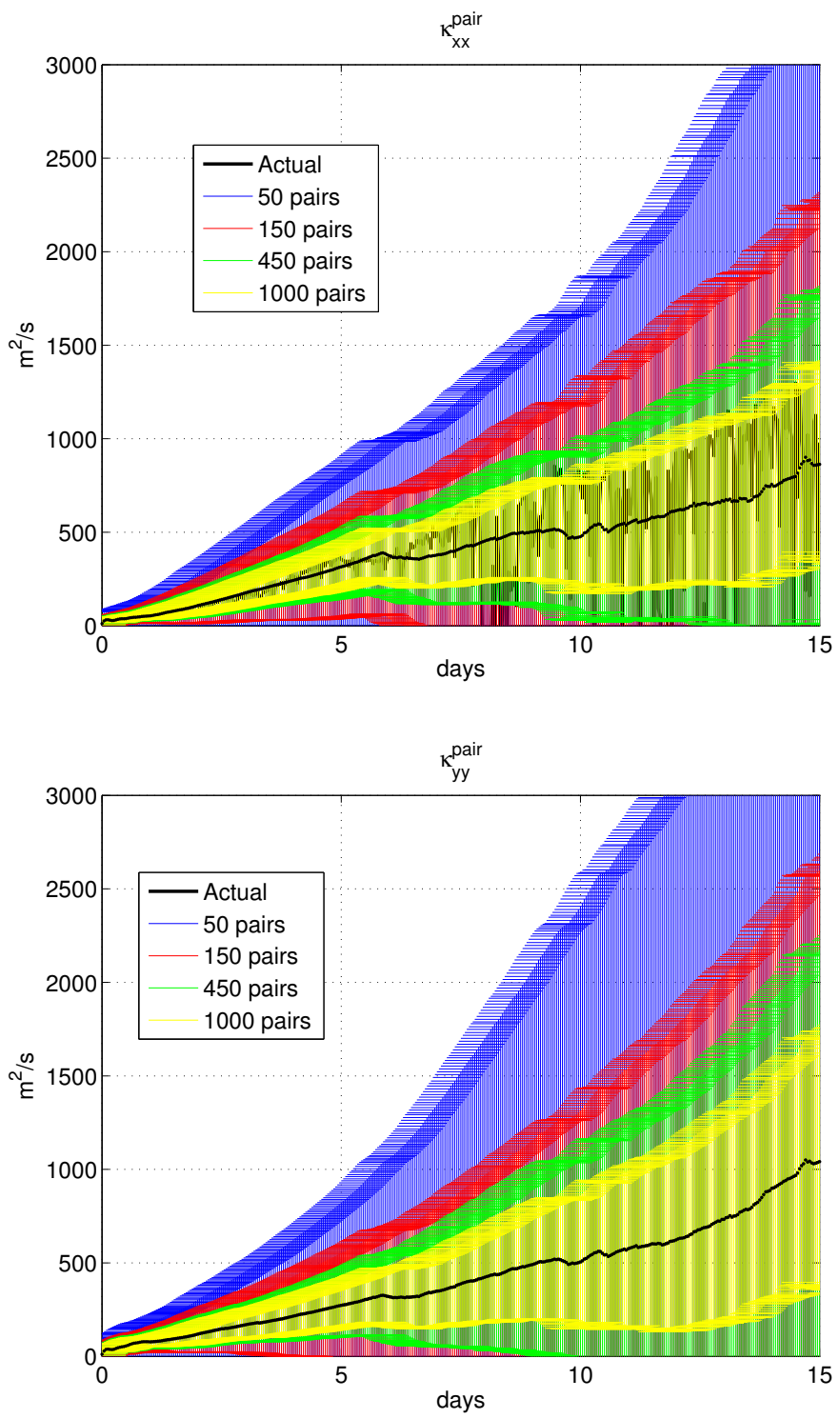

Figure 5-31: Errors on diffusivity for pair statistics.

Similarly, we can compute the uncertainty estimates on the separation velocity. Obviously, the uncertainties are largest for the smallest number of drifter pairs. How does the number of drifter pairs change our interpretation of the data? Recall that in the observational dataset ( 80 drifter pairs) we saw shorter time and space scales than in the model drifters (8000 drifter pairs), and recall that the scales were estimated by comparing when the separation velocity reached the single-particle velocity value. For smaller numbers of pairs, there will be larger uncertainties, and it will be more likely that the separation velocity will reach the single-particle value at shorter time 
and space scales than if we were to use more drifter pairs. The small number of pairs in the observations implies large uncertainties there, and so the estimates for spatial and temporal scales from the observations are not inconsistent with those of the model.
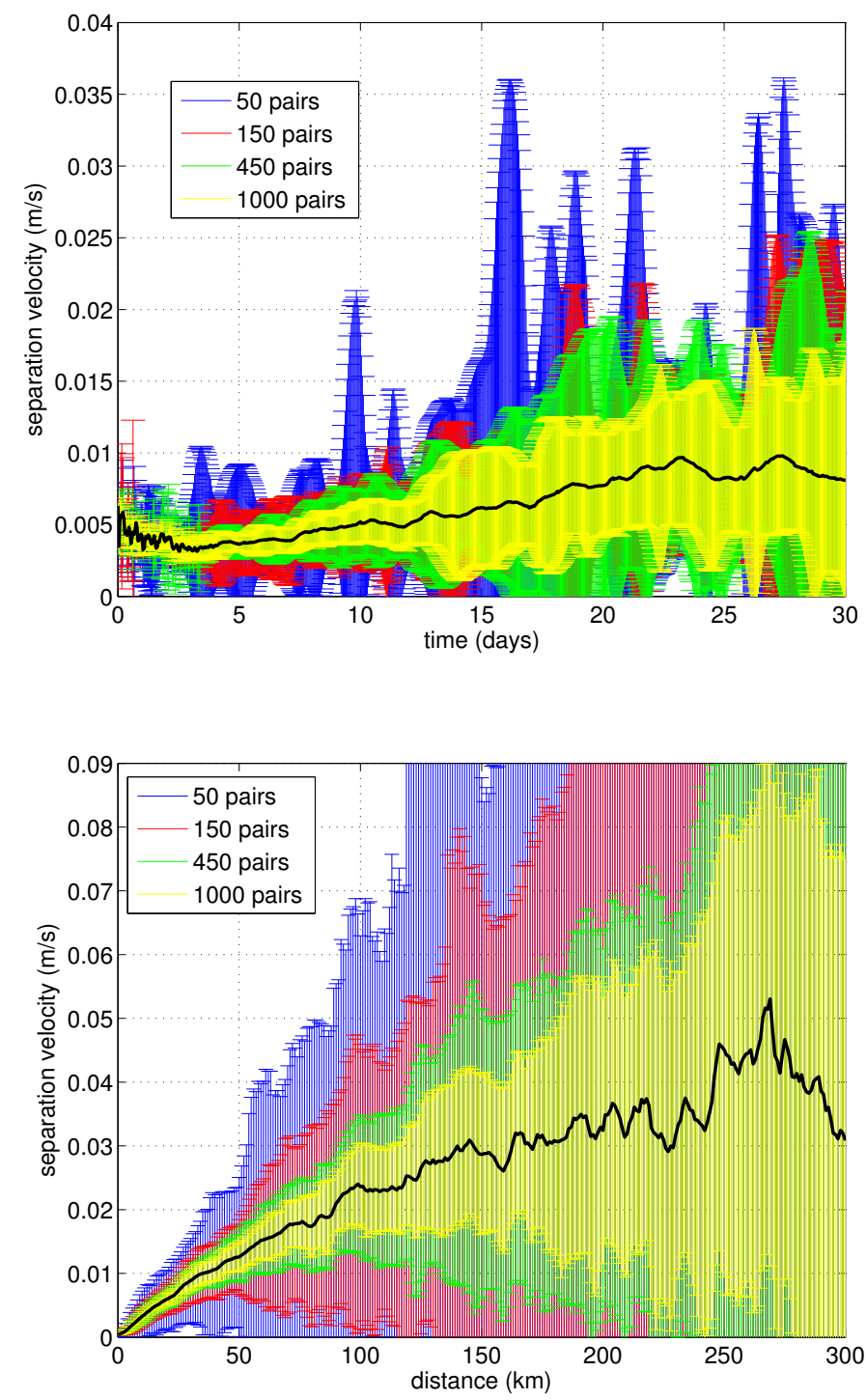

Figure 5-32: Errors on separation velocity for pair statistics. 


\subsection{Discussion and conclusion}

There were four different aspects of the analysis in chapter 4 that we evaluated in this chapter: calculation of the mean flow and eddy kinetic energy (EKE), the processing techniques used on the Lagrangian data, and estimating the eddy diffusivity and its uncertainty. In this section we briefly summarize the findings of the different sections. Processing and analyzing Lagrangian data and calculating the mean flow and EKE

In sections 5.4 and 5.5, we evaluated the processing and analysis methodology typically employed for Lagrangian data. The adaptive clustering method was shown to be preferable to that of the standard gridding approach in two key ways: the standard errors on the velocity are smaller, and the locations of the averaged data are a better representation of where the data were actually collected. Lagrangian observations are by nature not at fixed locations, but are spread out unevenly through the domain. Forcing this data to a standard, rectangular picture is tempting, but is misleading in that a single data point in one bin appears on first glance to have as much value as a bin containing many observations. The clustering approach only shows data where it exists.

The process of taking Lagrangian positions and obtaining useful information about the flow involves many assumptions and approximations, some of which may introduce bias to the calculation. We discussed a couple of different methods to compute velocity from the drifter positions (first-differences and cubic spline fitting), but there are others that, in other implementations and datasets, may be more appropriate. Also, we only filtered the position data to remove low-frequency fluctuations. Since at best the drifter data were hourly, we may not be fully resolving the temporally- and spatially-varying tidal motions, which makes removing the tides a difficult business. With better resolution on the position data, it may be possible to perform higher-order de-tiding of the position data, which would allow us to focus on higher-frequency dispersive motions not associated with the tides (such as inertial oscillations). More work and observational effort, in particular collecting drifter data where the drifters report 
their positions at much more frequent intervals, is required to determine whether this is a possibility.

\section{Diffusivity from model drifters vs. observations}

It was shown in section 5.6 that the model drifter diffusivity shows patterns largely consistent with the observations. Given that the model seems to be fairly reliable, we can now use it to speculate on what these measurements mean for copepod aggregations. The CC bin is where most right whales (and, by inference, more copepod patches) may be found, so let us focus our discussion there. The zonal component of $\kappa$ in this bin is larger than any of the other bins (figure 5-19), the uncertainties for a given number of drifters are largest (figure 5-28), and there is evidence for short spaceand time-scale motion here not present in the other bins (figure 5-22). It is tempting to conclude from these results that the $\mathrm{CC}$ bin is the least likely to contain coherent copepod patches, but closer inspection reveals this may not be the case. Recall that the effective resolution of the drifter data is on the order of the approximate scale of observed copepod patches. The apparent presence of diffusive motion in the $\mathrm{CC}$ bin could be on larger scales than the copepod patches, and in fact be the motion that generates the observed copepod patches.

\section{Estimating uncertainty of $\kappa$}

Uncertainty in $\kappa$ depends crucially upon the number of drifters, analogous to the results of Klocher et al. (2012). This work is the first to my knowledge to attempt to quantify uncertainty in $\kappa_{\text {pair }}$. We have already discussed that pair diffusivity and single-particle diffusivity don't quite measure the same effect in the flow. How do the uncertainties compare with one another?

Comparing the uncertainty estimates for single-particle vs. particle-pair diffusivity calculated in section 5.7 is difficult, because 1000 drifters does not necessarily equate to 1000 drifter pairs. For example, in the observational dataset, there are 370 drifters and 80 drifter pairs, while in the model drifters there are almost 8000 pairs for 4733 drifters. Uncertainties for 1000 drifters and 1000 drifter pairs are comparable at lags 
between 5 and 10 days. However, in the observational dataset, the uncertainties are going to be larger for the drifter pairs simply due to the small number of pairs. Since drifter pairs provide a more reliable measure of the spreading in the flow, the best strategy may be to release drifters in groups, thereby increasing the number of drifter pairs and reducing the uncertainty in the pair estimates.

What do these uncertainty estimates mean for the calculation of $\kappa$ ? To calculate the single-particle diffusivity, one integrates the velocity autocorrelation to infinity. Practically, however, we have seen that the uncertainty grows in time for longer lags. This suggests an intermediate time scale over which $\kappa$ may be reasonably estimated, somewhere between 5 and 10 days (longer than the Lagrangian decorrelation time scale but short enough such that the uncertainties remain as low as possible. The fact that there is a range of time scales indicates that there is also a range of spatial scales over which it is appropriate to measure $\kappa$, as the drifters used to estimate $\kappa$ travel over a range of distances during the 5-10 day time frame. Depending upon the region in the GOM, the spatial scale over which $\kappa$ may be reasonably estimated can range from $15 \mathrm{~km}$ where the flow speed is slow (like in the interior GOM), to up to $200 \mathrm{~km}$ where the flow speed is fast (e.g. coastal current or northern flank of Georges Bank). Similar scales may apply to the pair diffusivity, although the upper range of these scales may be limited by the dominant scale of the eddies (above which the pairs are no longer correlated, see figure 5-17).

The take-home points of this thesis chapter are:

- The adaptive clustering method reduces the standard error of velocity estimates while still resolving the major features of the flow in the GOM. In addition, because the data appears where it is collected (as opposed to forced onto a rigid grid), we are able to notice subtle but perhaps important differences in the flow (namely, in the GSC, figure 5-4).

- Mean flow and EKE from the model, model drifters, and observations are all consistent with one another (figure 5-6). Main differences between model and 
model drifters are likely due to drifter processing (namely detiding and computing velocity from first difference of drifter position, figures 5-10 and 5-11).

- Diffusivity estimates from model drifters in the GOM and three bins have smaller standard errors due to larger number of drifters and are largely consistent with observations (figures 5-14 and 5-19).

- Pair diffusivity estimates calculated from model drifters agree with observations until about 20 days, after which the model estimates continue to increase while the observations become dominated by noise (figure 5-16). Pair separation statistics are characterized by slightly larger time and space scales when compared to data (figure 5-17).

- Pair statistics calculated in three bins reveals structure at short time (less than 10 days) and small space (less that $50 \mathrm{~km}$ ) scales in the $\mathrm{CC}$ bin not reflected in other bins (figures 5-21 and 5-22), which could be important for copepod aggregations.

- Computing $\kappa$ from Green's functions method yields result largely in agreement with $\kappa_{E u l}$ (figure 5-23).

- Error estimates from bootstrapping show that estimates for $\kappa$ over the entire GOM can be made more accurate with more drifters, but that approximate estimates can be reliably made with as few at 50 drifters (figure 5-24).

- Differentiating spatial structure in single-particle $\kappa$, however, is more difficult, yet can be achieved with 400 drifters (figures 5-25, 5-26, 5-27 and 5-29).

- Pair dispersion uncertainties for 1000 drifter pairs are comparable to that of single-particle estimates for 1000 drifters (5-31). 


\section{Chapter 6}

\section{Discussion and conclusions}

This thesis takes several steps towards understanding the circulation in the southwestern Gulf of Maine and what role that circulation may play in the dynamics of dense copepod aggregations. The four thesis body chapters address different problems in understanding this circulation, and there were several key findings unique to this work. This chapter summarizes those key findings in section 6.1. While this thesis makes clear many aspects of the circulation as pertains to copepod patches, there are many unresolved problems and questions. Section 6.2 discusses these problems and questions, and suggests some future work towards understanding the dynamics of copepod patches, which is followed by concluding remarks in section 6.3.

\subsection{Major contributions of this thesis}

The work in chapter 2 discusses in detail the structure of nearshore freshwater in the southwestern GOM. This chapter, to my knowledge, is the first to quantify the variability of the coastal freshwater in the southwestern GOM using shipboard underway TSG observations, and to attempt to understand the forcing causing this variability on time scales ranging from interannual to synoptic. The seasonal variability in river discharge leads to more (and more intense in terms of the magnitude of the gradient) surface salinity fronts. It was also shown that alongshore wind may play a 
role in moving these salinity fronts around on short (synoptic) time scales. However the exact role the wind plays is more complicated than can be explained by current theory (Lentz (2004) and Moffat and Lentz (2011)). The importance of the salinity front and freshwater plumes in possibly generating copepod aggregations, which is expanded upon in chapter 3, means that the position and variability of the salinity front may be important in determining the resulting distribution of right whales and other organisms that feed upon these dense patches.

Chapter 3 expands upon the work from Epstein and Beardsley (2001) in a few key ways. First, this work considers the effect of a dynamic front with along-front structure, rather than the simple two-dimensional, kinematic picture of previous studies; this resulted in some important differences in the resulting copepod distribution. Notably, the concentration of copepods at the front increased more slowly. Also, the importance of the plume-front-relative circulation was emphasized, which can effectively remove copepods from the front, inhibiting concentration growth while spreading copepods throughout the plume. The effect of different copepod behaviors, including isopycnal-following, was shown to change the resulting copepod distribution. Instead of a dense patch forming near the plume front, isopycnal copepods were subducted under the plume and were compressed in the vertical. The implication for copepod patches in the GOM is that whales might not expect to find patches necessarily right at the plume front, but somewhere near it or perhaps under the plume entirely.

The variability in the Lagrangian characteristics of the GOM was explored in chapter 4. The results of this chapter do not point to some component of the GOM circulation that generates aggregations, but rather looks at quantifying the ability of the flow to destroy a patch. This chapter updated the work of Manning et al. (2009) in yielding the highest to-date resolution pictures of GOM summertime mean currents and EKE. This high resolution was obtained by utilizing a new, adaptive clustering technique (Koszalka and Lacasce (2010)), which reduced standard error over previous methods. In addition, this chapter provides the most complete picture 
of eddy diffusivity in the GOM currently available.

The work of chapter 4 was supplemented in chapter 5 by using numerical drifters in a realistic FVCOM simulation of the GOM circulation. These drifters were used to evaluate how well the Lagrangian techniques recreate the circulation; these results are not special to the GOM, but apply to any Lagrangian study. Also, this is one of the first and most complete comparisons between a numerical model, numerical drifters in that model, and Lagrangian observations. These results validate the model against the observations as well as the particle tracking algorithm against the model. While the particle tracking did on the whole agree well with the model, there were a few differences between the two, which highlighted how the processing of Lagrangian data can sometimes bias the resulting calculations. The uncertainty estimates for the eddy diffusivity are particularly enlightening in that they provide concrete limits for how well the eddy diffusivity is known, which is important when trying to understand structure in $\kappa$ which may be important to copepod aggregations.

\subsection{Future work}

The application of the hypothetical models of Lentz (2004) and Moffat and Lentz (2011) to the freshwater plume off Cape Cod showed that, while the models do a fairly good job of predicting movement of the offshore edge of the plume, these theories fail to predict the observed changes in density, cross-sectional area, or depth of the plume. The likely culprit for this discrepancy is the assumption in the theory of along-shore uniformity. The coastline of the southwestern GOM is highly variable, with lots of bays, inlets, harbors, islands, and topography, all of which create along-shore variability which may lead to entrainment or detrainment of fluid out of the plume or instability processes which cannot exist in a simple, two-dimensional theoretical model. In order to fully understand this system, a fully three-dimensional theory must be developed which can account for these processes.

The idealized modeling in chapter 3 suggests very specific interactions between 
the dynamics of freshwater plumes and copepod behaviors which can lead to increases in copepod concentration at the boundary between dense and light fluid, but do these interactions occur in the wild? Other than the single anecdotal acoustic backscatter section in Epstein and Beardsley (2001), there is little evidence in the literature of this process occurring in the southwestern GOM. Targeted observational experiments focused on buoyant plume fronts should be a priority for future work on this topic. In particular, coincident measurements of three-dimensional circulation, density structure, and the location/movement of copepods are necessary to understand this mechanism. The circulation in a reference frame moving with the plume nose is of particular interest here, since this circulation was pivotal in organizing the copepods, and is not well understood in real systems. On the modeling side, this work only considers a hydrostatic model of a coastal river plume with parameterizations for non-hydrostatic processes. A fully non-hydrostatic model, similar to that of Helfrich and Pineda (2003) but including rotation and bathymetric effects, has not been used in a study such as this, and may indeed lead to new new insight into the movement of particles near the nose of buoyant plumes.

The SCOPEX project posited three hypotheses to explain the high copepod concentrations in the southwestern GOM, which we will now revisit. The first hypothesis was that there was that in situ growth of copepod abundance as a result of locally enhanced primary productivity. The SCOPEX results showed no evidence for this hypothesis, nor has any of the work presented herein. The second hypothesis suggested that some undetermined physical and physical-biological processes were likely responsible for the high concentration of copepods in patches observed in the southwestern GOM (Kenney and Wishner (1995)). Chapter 3's results show that the copepod concentration can be enhanced by about a factor of 10 above the ambient concentration. Observations, however, indicate that copepod concentrations may be as much as 100-1000 higher than the ambient (Baumgartner et al. (2003)). This prompts an obvious question: what results in the additional observed amplification of copepod concentration? Additional work with the idealized models may indicate that the pro- 
posed plow mechanism may be capable of producing significantly larger concentration enhancement, while the observational work proposed may reveal additional processes occurring along the edge of the plume which provides additional enhancement. The third hypothesis, that there is a behavioral tendency to form dense patches, was not directly addressed by SCOPEX, nor in this work. However, work by Davis et al. (1991) has shown that social behavior can lead to patchiness in copepods. Social behavior has been studied in krill (Verdy and Flierl (2008)); further work to understand social interactions between copepods may prove to be important in the southwestern GOM.

The Lagrangian characteristics of the flow described from observations in chapter 4 and from the model in chapter 5 showed in general good agreement. The uncertainty estimates on $\kappa$ in chapter 5 , however, showed how sensitive some of these estimates are on the number of realizations, or drifters, in the flow. It is necessary to continue to deploy new Lagrangian instruments, especially in the high flow-through regions such as the coastal current and Georges Bank regions in order to reduce uncertainty. Also, the seasonal bias in the observations makes winter-time estimates of even the most simple quantities almost impossible. Understanding even the basic seasonal variability in the surface circulation requires significant increases in the wintertime drifter sampling. Additionally, continuing to use and improve models of the yearround circulation in the GOM would help to fill in this gap in information. Finally, the scales resolved by the current drifters are larger than typical copepod patch sizes, so drawing important inferences about copepod patches from these data is a difficult task. Future observational programs targeting the problem of copepod patchiness should consider higher sampling rates for Lagrangian drifters to understand dispersion on small time and space scales important to copepod patches. 


\subsection{Concluding remarks}

It is my hope that this thesis work will inspire new field experiments and observational programs. Recently, changes in the Atlantic Cod population have caused regulators to make drastic changes to the allowed catch in the GOM/Northwest Atlantic fisheries, costing local fishermen lots of money. The ecosystem continues to change; it is imperative that we understand the system so that we may understand these changes and adapt accordingly. New technology, including optical plankton counters, video plankton recorders, and broadband acoustic systems allow for previously unattainable high spatial- and temporal-resolution pictures of phytoplankton, copepods, and fish (Lawson et al. (2007), Wiebe et al. (2013)). Autonomous gliders and other AUVs are able to map three-dimensional physical and biological fields rapidly in time and space (Rudnick et al. (2004), Baumgartner and Fratantoni (2008)). Surface currents can be mapped from shore by high-frequency radar on scales smaller than $1 \mathrm{~km}$ (Kirincich et al. (2012)). Moving forward, using this new technology may be the best way to solve this important problem. 


\section{Bibliography}

Anderson, D. M., B. A. Keafer, W. R. Geyer, R. P. Signell, and T. C. Loder, 2005: Toxic alexandrium blooms in the western gulf of maine: The plume advection hypothesis revisited. Limnology and Oceanography, 50 (1), 328-345.

Balch, W., D. Drapeau, B. Bowler, and T. Huntington, 2012: Step-changes in the physical, chemical and biological characteristics of the gulf of maine, as documented by the gnats time series. Marine Ecology Progress Series, 450, 11-35.

Baumgartner, M., C. Mayo, and R. Kenney, 2007: Enormous carnivores, microscopic food, and a restaurant that's hard to find. The urban whale: North Atlantic right whales at the crossroads. Harvard University Press, Cambridge, MA, 138-171.

Baumgartner, M. F., T. V. Cole, P. J. Clapham, and B. R. Mate, 2003: North atlantic right whale habitat in the lower bay of fundy and on the sw scotian shelf during 1999-2001.

Baumgartner, M. F. and D. M. Fratantoni, 2008: Diel periodicity in both sei whale vocalization rates and the vertical migration of their copepod prey observed from ocean gliders. Limnology and Oceanography, 53 (5), 2197-2209.

Baumgartner, M. F., N. S. Lysiak, C. Schuman, J. Urban-Rich, and F. W. Wenzel, 2011: Diel vertical migration behavior of calanus finmarchicus and its influence on right and sei whale occurance. Marine Ecology Progress Series, 423, 167-184.

Baumgartner, M. F. and B. R. Mate, 2003: Summertime foraging ecology of north atlantic right whales. Marine Ecology Progress Series, 264, 123-135.

Beardsley, R., A. Epstein, C. Chen, K. Wishner, M. Macaulay, and R. Kenney, 1996: Spatial variability in zooplankton abundance near feeding right whales in the Great South Channel. Deep Sea Research Part II: Topical Studies in Oceanography, 43, 1601-1625, doi:10.1016/S0967-0645(96)00050-1.

Beardsley, R., R. Limeburner, and L. Rosenfeld, 1985: Introduction to the code-2 moored array and large-scale data report, in code-2: Moored array and large-scale data report. Tech. Report, 85-35, Woods Hole Oceanographic Institution, $1-21$. 
Brink, K. H., R. Limeburner, and R. C. Beardsley, 2003: Properties of flow and pressure over Georges Bank as observed with near-surface drifters. Journal of Geophysical Research (Oceans), 108, 8001.

Buchanan, C., B. Goldberg, and R. McCartney, 1982: A laboratory method for studying zooplankton swimming behaviors. Hydrobiologia, 94 (1), 77-89.

Chao, S., 1987: Wind-driven motion near inner shelf fronts. Journal of Geophysical Research, 92, 3849-3860, doi:10.1029/JC092iC04p03849.

Chen, C., R. Beardsley, and G. Cowles, 2006: An unstructured-grid, finite-volume coastal ocean model (fvcom) system. Oceanography, 19 (1), 12.

Chen, C., R. C. Beardsley, S. Hu, Q. Xu, and H. Lin, 2005: Using MM5 to Hindcast the Ocean Surface Forcing Fields over the Gulf of Maine and Georges Bank Region*. Journal of Atmospheric and Oceanic Technology, 22, 131, doi:10.1175/JTECH1682.1 .

Chen, C., R. C. Beardsley, and R. Limeburner, 1995: Variability of water properties in late spring in the northern Great South Channel. Continental Shelf Research, 15, 415-431, doi:10.1016/0278-4343(94)00054-Q.

Chen, C., H. Liu, and R. Beardsley, 2003: An Unstructured Grid, Finite-Volume, Three-Dimensional, Primitive Equations Ocean Model: Application to Coastal Ocean and Estuaries. Journal of Atmospheric and Oceanic Technology, 20, 159-+.

Churchill, J. H., J. Runge, and C. C., 2011: Processes controlling retention of springspawned atlantic cod (gadus morhua) in the western gulf of maine and their relationship to an index of recruitment success. Fisheries Oceanography, 20 (1), 32-46.

Davis, C. S., G. R. Flierl, P. H. Wiebe, and P. J. S. Franks, 1991: Micropatchiness, turbulence and recruitment in plankton. Journal of Marine Research, 49, 109-151.

Davis, R. E., 1985: Drifter observations of coastal surface currents during CODE: The statistical and dynamical views. Journal of Geophysical Research, 90, 4756-4772.

Davis, R. E., 1991: Observing the general circulation with floats. Deep Sea Research Part A. Oceanographic Research Papers, 38 (Suppl. 1), S531-S571.

Durbin, E., J. Runge, R. Campbell, P. Garrahan, M. Casas, and S. Plourde, 1997: Late fall-early winter recruitment of calanus finmarchicus on georges bank. Marine Ecology Progress Series, 151 (1), 103-114.

Epstein, A. W., 1995: Physical processes and zooplankton distribution in the great south channel: Observational and numerical studies. Tech. rep., MIT/WHOI Joint Program in Physical Oceanography. 
Epstein, A. W. and R. C. Beardsley, 2001: Flow-induced aggregation of plankton at a front: a 2-d eulerian model study. Deep Sea Research Part II: Topical Studies in Oceanography, 48 (1-3), 395 - 418, coupled biological and physical studies of plankton populations on Georges Bank and related North Atlantic regions.

Fairall, C. W., E. F. Bradley, D. P. Rogers, J. B. Edson, and G. S. Young, 1996: Bulk parameterization of air-sea fluxes for tropical ocean-global atmosphere coupledocean atmosphere response experiment. Journal of Geophysical Research, 101, $3747-3764$.

Fong, D., W. Geyer, and R. Signell, 1997: The wind-forced response on a buoyant coastal current: Observations of the western gulf of maine plume. Journal of Marine Systems, 12 (1-4), $69-81$.

Fong, D. A. and W. R. Geyer, 2001: Response of a river plume during an upwelling favorable wind event. Journal of Geophysical Research, 106, 1067-1072, doi:10.1029/2000JC900134.

Fong, D. A. and W. R. Geyer, 2002: The alongshore transport of freshwater in a surface-trapped river plume*. Journal of Physical Oceanography, 32 (3), 957-972.

Franks, P. J. S., 1992: Sink or swim: accumulation of biomass at fronts. Marine Ecology Progress Series, 82, 1-12.

Fry, B., 1988: Food web structure on georges bank from stable c, n, and s isotopic compositions. Limnology and oceanography, 1182-1190.

Genin, A., J. S. Jaffe, R. Reef, C. Ricther, and P. J. S. Franks, 2005: Swimming against the flow: A mechanism of zooplankton aggregation. Science, 308 (860), 4.

Griffiths, R. W., 1986: Gravity currents in rotating systems. Annual Review of Fluid Mechanics, 18, 59-89.

Halpin, P., et al., 2006: Obis-seamap: developing a biogeographic research data commons for the ecological studies of marine mammals, seabirds, and sea turtles. Marine Ecology Progress Series, 316.

Hannah, C., C. Naimie, J. Loder, and F. Werner, 1997: Upper-ocean transport mechanisms from the gulf of maine to georges bank, with implications for calanus supply. Continental Shelf Research, 17 (15), 1887 - 1911.

Haury, L. R., P. H. Wiebe, M. H. Orr, and M. G. Briscoe, 1983: Tidally generated high-frequency internal wave packets and their effects on plankton in massachusetts bay. Journal of Marine Research, 41 (1), 65-112.

He, R., D. J. McGillicuddy, D. R. Lynch, K. W. Smith, C. A. Stock, and J. P. Manning, 2005: Data assimilative hindcast of the gulf of maine coastal circulation. Journal of Geophysical Research: Oceans (1978-2012), 110 (C10). 
Helfrich, K. R. and J. Pineda, 2003: Accumulation of particles in propagating fronts. Limnology and oceanography, 1509-1520.

Hetland, R. D. and R. P. Signell, 2005: Modeling coastal current transport in the gulf of maine. Deep Sea Research Part II: Topical Studies in Oceanography, 52 (19-21), 2430 - 2449, doi:DOI: 10.1016/j.dsr2.2005.06.024, URL http://www.sciencedirect.com/science/article/B6VGC-4H74MOK-1/2/10384f cc336c3cc6a4d9 the Ecology and Oceanography of Toxic Alexandrium fundyense Blooms in the Gulf of Maine.

Kenney, R. D. and K. F. Wishner, 1995: The South Channel Ocean Productivity EXperiment. Continental Shelf Research, 15, 373-384.

Kirincich, A. R., T. de Paolo, and E. Terrill, 2012: Improving hf radar estimates of surface currents using signal quality metrics, with application to the mvco highresolution radar system. Journal of Atmospheric and Oceanic Technology, 29 (9), $1377-1390$.

Klocher, A. R., R. Ferrari, J. H. Lacasce, and S. T. Merrifield, 2012: Reconciciling float-based and tracer-based estimates of eddy diffusivities. Journal of Marine Research, in press.

Koszalka, I. M. and J. H. Lacasce, 2009: Relative dispersion in the nordic seas. Journal of Marine Research, 67, 411-433.

Koszalka, I. M. and J. H. Lacasce, 2010: Lagrangian analysis by clustering. Ocean Dynamics, 60, 957-972.

Lacasce, J. H., 2008: Lagrangian statistics from oceanic and atmospheric observations. Lecture Notes in Physics, 744, 165-218.

Lalli, C. M. and T. R. Parsons, 1997: Biological oceanography: an introduction. Butterworth-Heinemann.

Large, W. G. and S. Pond, 1981: Open Ocean Momentum Flux Measurements in Moderate to Strong Winds. Journal of Physical Oceanography, 11, 324-336.

Lawson, G. L., T. K. Stanton, and P. H. Wiebe, 2007: Characterizing variability in the distribution of high-frequency acoustic backscattering in a shallow water coastal region. Tech. rep., DTIC Document.

Lentz, S., 2004: The Response of Buoyant Coastal Plumes to Upwelling-Favorable Winds*. Journal of Physical Oceanography, 34, 2458-+, doi:10.1175/JPO2647.1.

Lentz, S. J. and K. R. Helfrich, 2002: Buoyant gravity currents along a sloping bottom in a rotating fluid. Journal of Fluid Mechanics, 464, 251-278. 
Lentz, S. J. and J. Largier, 2006: The Influence of Wind Forcing on the Chesapeake Bay Buoyant Coastal Current*. Journal of Physical Oceanography, 36, 1305-+, doi:10.1175/JPO2909.1.

Lenz, P., A. Hower, and D. Hartline, 2004: Force production during pereiopod power strokes in calanus finmarchicus. Journal of marine systems, 49 (1), 133-144.

Li, Y., R. He, and D. J. McGillicuddy Jr, 2013: Seasonal and interannual variability in gulf of maine hydrodynamics: 2002-2011. Deep Sea Research Part II: Topical Studies in Oceanography.

Loder, J. W., 1980: Topographic Rectification of Tidal Currents on the Sides of Georges Bank. Journal of Physical Oceanography, 10, 1399-1416.

Lumpkin, R., A.-M. Treguier, and K. Speer, 2002: Lagrangian Eddy Scales in the Northern Atlantic Ocean. Journal of Physical Oceanography, 32, 2425-2440.

Magaldi, M. G., T. M. Özgökmen, A. Griffa, and M. Rixen, 2010: On the response of a turbulent coastal buoyant current to wind events: the case of the Western Adriatic Current. Ocean Dynamics, 60, 93-122, doi:10.1007/s10236-009-0247-9.

Manning, J. P., D. J. McGillicuddy, Jr., N. R. Pettigrew, J. H. Churchill, and L. S. Incze, 2009: Drifter observations of the Gulf of Maine Coastal Current. Continental Shelf Research, 29, 835-845, doi:10.1016/j.csr.2008.12.008.

Miller, C. B., D. R. Lynch, F. Carlotti, W. Gentleman, and C. V. W. Lewis, 1998: Coupling of an individual-based population dynamic model of calanus finmarchicus to a circulation model for the georges bank region. Fisheries Oceanography, 7 (3-4), $219-234$

Moffat, C. and S. Lentz, 2011: On the response of a buoyant plume to downwellingfavorable wind stress. Submitted.

Naimie, C., R. Limeburner, C. Hannah, and R. Beardsley, 2001: On the geographic and seasonal patterns of the near-surface circulation on georges bank from real and simulated drifters. Deep Sea Research Part II: Topical Studies in Oceanography, 48, 501-518.

Niiler, P., 2009: Ageostrophic circulation in the ocean. Oceanography in 2025: Proceedings of a Workshop, National Academies Press, 73.

North, E., E. Adams, Z. Schlag, C. Sherwood, R. He, K. Hyun, and S. Socolofsky, 2011: Simulating oil droplet dispersal from the deepwater horizon spill with a lagrangian approach. Geophysical Monograph Series, 195, 217-226.

North, E., R. Hood, S. Chao, and L. Sanford, 2006: Using a random displacement model to simulate turbulent particle motion in a baroclinic frontal zone: A new 
implementation scheme and model performance tests. Journal of Marine Systems, 60 (3), 365-380.

Nøttestad, L. and T. Similä, 2001: Killer whales attacking schooling fish: Why force herring from deep water to the surface? Marine mammal science, 17 (2), 343-352.

Olson, D. B. and R. H. Backus, 1985: The concentrating of organisms at fronts: A cold-water fish and a warm-core gulf stream ring. Journal of Marine Research, 43 (1), 113-137.

Ostrand, W. D., S. Howlin, and T. A. Gotthardt, 2004: Fish school selection by marbled murrelets in prince william sound, alaska: responses to changes in availability. Marine Ornithology, 32, 69-76.

Pettigrew, N. R., et al., 2005: The kinematic and hydrographic structure of the gulf of maine coastal current. Deep Sea Research Part II: Topical Studies in Oceanography, 52 (19), 2369-2391.

Pitcher, K. W. and D. G. Calkins, 1981: Reproductive biology of steller sea lions in the gulf of alaska. Journal of Mammalogy, 599-605.

Pollard, R. T., P. B. Rhines, and R. O. R. Y. Thompson, 1972: The deepening of the wind-Mixed layer. Geophysical and Astrophysical Fluid Dynamics, 4, 381-404.

Rudnick, D. L., R. E. Davis, C. C. Eriksen, D. M. Fratantoni, and M. J. Perry, 2004: Underwater gliders for ocean research. Marine Technology Society Journal, 38 (2), 73-84.

Schlag, Z. R. and E. W. North, 2012: Lagrangian transport (ltrans) v.2 model user's guide. Tech. Rep. 183p, University of Maryland Center for Environmental Science Horn Point Lab.

Schoenherr, J. R., 1991: Blue whales feeding on high concentrations of euphausiids around monterey submarine canyon. Canadian Journal of Zoology, 69 (3), 583594.

Shchepetkin, A. and J. McWilliams, 2005: The regional oceanic modeling system (roms): a split-explicit, free-surface, topography-following-coordinate oceanic model. Ocean Modelling, 9, 347-404.

Shcherbina, A. Y. and G. G. Gawarkiewicz, 2008a: A coastal current in winter: 2. Wind forcing and cooling of a coastal current east of Cape Cod. Journal of Geophysical Research (Oceans), 113 (12), 10 014-+, doi:10.1029/2008JC004750.

Shcherbina, A. Y. and G. G. Gawarkiewicz, 2008b: A coastal current in winter: Autonomous underwater vehicle observations of the coastal current east of Cape Cod. Journal of Geophysical Research (Oceans), 113 (12), 7030-+, doi: 10.1029/2007JC004306. 
Sims, D. W. and V. A. Quayle, 1998: Selective foraging behaviour of basking sharks on zooplankton in a small-scale front. Nature, 393 (6684), 460-464.

Sutherland, D. A. and R. S. Pickart, 2008: The east greenland coastal current: Structure, variability, and forcing. Progress In Oceanography, 78 (1), 58 - 77, doi:DOI: 10.1016/j.pocean.2007.09.006.

Swenson, M. S. and P. P. Niiler, 1996: Statistical analysis of the surface circulation of the California Current. Journal of Geophysical Research, 101, 22 631-22 646.

Tarrant, A. M., M. F. Baumgartner, T. Verlycke, and C. L. Johnson, 2008: Differential gene expression in diapausing and active calanus finmarchicus (copepoda). Marine Ecology Progress Series, 355, 193-207.

Verdy, A. and G. Flierl, 2008: Evolution and social behavior in krill. Deep Sea Research Part II: Topical Studies in Oceanography, 55 (3), 472-484.

Werner, S., R. Beardsley, S. Lentz, D. Hebert, and N. Oakey, 2003: Observations and modeling of the tidal bottom boundary layer on the southern flank of georges bank. Journal of geophysical research, 108 (C11), 8005.

Whitney, M. M. and R. W. Garvine, 2005: Wind influence on a coastal buoyant outflow. Journal of Geophysical Research (Oceans), 110, 3014-+, doi: 10.1029/2003JC002261.

Wiebe, P. H., G. L. Lawson, A. C. Lavery, N. J. Copley, E. Horgan, and A. Bradley, 2013: Improved agreement of net and acoustical methods for surveying euphausiids by mitigating avoidance using a net-based led strobe light system. ICES Journal of Marine Science: Journal du Conseil.

Wishner, K. F., J. R. Schoenherr, R. Beardsley, and C. Chen, 1995: Abundance, distribution and population structure of the copepod Calanus finmarchicus in a springtime right whale feeding area in the southwestern Gulf of Maine. Continental Shelf Research, 15, 475-507, doi:10.1016/0278-4343(94)00057-T.

Wood, C. L., 2008: The interaction of two coastal plumes and its effect on the transport of alexandrium fundyense. M.S. thesis, Massachusetts Institute of Technology.

Yankovsky, A. and D. Chapman, 1997: A Simple Theory for the Fate of Buoyant Coastal Discharges*. Journal of Physical Oceanography, 27, 1386-1401.

Zhurbas, V. M. and I. S. Oh, 2003: Lateral diffusivity and Lagrangian scales in the Pacific Ocean as derived from drifter data. EGS - AGU - EUG Joint Assembly, 4112. 